University of Louisville

ThinkIR: The University of Louisville's Institutional Repository

Electronic Theses and Dissertations

8-2015

\title{
The local Cygnus cold cloud and further constraints on a local hot bubble.
}

Geoffrey Robert Lentner

University of Louisville

Follow this and additional works at: https://ir.library.louisville.edu/etd

Part of the Physics Commons

\section{Recommended Citation}

Lentner, Geoffrey Robert, "The local Cygnus cold cloud and further constraints on a local hot bubble." (2015). Electronic Theses and Dissertations. Paper 2213.

https://doi.org/10.18297/etd/2213

This Master's Thesis is brought to you for free and open access by ThinkIR: The University of Louisville's Institutional Repository. It has been accepted for inclusion in Electronic Theses and Dissertations by an authorized administrator of ThinkIR: The University of Louisville's Institutional Repository. This title appears here courtesy of the author, who has retained all other copyrights. For more information, please contact thinkir@louisville.edu. 


\title{
THE LOCAL CYGNUS COLD CLOUD AND FUTHER CONSTRAINTS ON A LOCAL HOT BUBBLE
}

\author{
By \\ Geoffrey Robert Lentner \\ B.S., Purdue University, 2013 \\ A Thesis \\ Submitted to the Faculty of the \\ College of Arts and Sciences of the University of Louisville \\ in Partial Fulfillment of the Requirements \\ for the Degree of
Master of Science
in Physics \\ Department of Physics and Astronomy \\ University of Louisville \\ Louisville, Kentucky
}

August 2015 
Copyright 2015 by Geoffrey Robert Lentner

All rights reserved 



\title{
THE LOCAL CYGNUS COLD CLOUD AND FUTHER CONSTRAINTS ON A LOCAL HOT BUBBLE
}

\author{
By \\ Geoffrey Robert Lentner \\ B.S., Purdue University, 2013 \\ A Thesis Approved On \\ July 20, 2015 \\ by the following Thesis Committee: \\ Thesis Director \\ James Lauroesch \\ Lutz Haberzettl \\ John Kielkopf \\ Ryan Gill
}




\section{DEDICATION}

This work is dedicated to my two beautiful boys, Henry and Charlie.

Their lives give mine meaning and motivation. 


\section{ACKNOWLEDGEMENTS}

One does not simply earn an advanced degree without an innumerable quantity of assists, both in life and in academics. There have been and continue to be individuals who selflessly promote and support me in the pursuit of my interests. Though I cannot possibly acknowledge every person who influenced me in a way that inexorably lead to my current situation, I want to highlight the contributions of many people that have been instrumental.

There will have been countless minute situations and occurrences that have shaped my growing mind to prepare me for what ultimately became a passion. Despite being what were completely unguided forces, I am thankful to have been born into a world and society where I am enabled to spend my life pursuing knowledge.

Two individuals, though, who indeed had my best interests at heart and mind were my mother and father. I would like to formally express my debt and gratitude to my parents Robert William Lentner III and Jennifer Anne Wedge. I suspect my sudden and spontaneous leap into natural science was not expected when it occurred, or at least my eventual decision to apply for graduate studies. I truly believe that throughout my life my parents have supported me in my decisions and interests, whatever they may be. I have always felt and continue to feel loved and encouraged by them. They will tell me how proud they are of what I've accomplished and what I will accomplish. I want to express a heartfelt thank you, to say that through it all I always felt supported.

There is another person from my childhood that I must recognize as being import to my growth, both intellectually and in other areas. We did not necessarily see eye-to-eye at times but as we grew older I saw him as an inspiration. My brother, Aaron David Lentner, is arguably one of the most import people to have helped me get where I am at the present moment. It is literally the case that I could not have pursued science as a career without 
his help and I am forever thankful for his vote of confidence in me when I needed it most.

I cannot neglect to acknowledge the love and support of my incredible wife, Whitney Leigh Lentner. She is my first and forever romance. She has been my encouragement even before I started on the path that lead to this thesis. Well into my undergraduate career I had the audacious idea to drop everything I had been working on and study physics. With all things in my life, she was the first person I came to. It was a complete upheaval and added much uncertainty to our future. She supported me then, and now. With our two beautiful boys Henry Francis Lentner and Charlie Alexander Lentner, graduate studies have been for me an especially rigorous challenge, particularly these past few months. I want to thank her for being such a caring and supportive wife and mother.

Academically, there are many individuals who contributed to my current success. There were several teaching assistants and professors who taught me both the substance of physics and what it really meant to be a scientist. In particular I want to mention two faculty from Purdue University that are directly responsible for getting me into a graduate program in physics. As a professor, Dr. Jaehyon Rhee peaked my motivation to pursue science. He provided my first research experience with nothing to go on but my passion. Without this initial spring board, I could not have ultimately joined the academic world. Also, Dr. Rebecca Lindell saw in me what at times I did not see myself. Without her support and constant confidence I would not have been accepted by a graduate school.

Here at the University of Louisville, many have given me both their support and collaboration. Chief among them is my advisor and mentor, Dr. James Lauroesch. We have made and will continue to make a great team. Both his intellect and kindness have gone unmatched. He has always taken an interest in my thoughts and ideas; in my two years in the department I have worked on many projects, even ones not related to our research into the ISM. It is often the case that graduate students are at the mercy of the advisor they find themselves working with. I can say with confidence that I could not have had a better advisor, intellectually or otherwise. Others have as well been in integral component to my experience. In particular, I want to acknowledge both the collaboration 
and constructive criticism of the faculty and graduate students in the astrophysics group. Wherever my career takes me, I will maintain a resolute bridge between me and the department that gave me my start.

This research is based on spectral data retrieved from the ELODIE archive at Observatoire de Haute-Provence (OHP) (Moultaka et al., 2004) and has made use of Matplotlib, a 2D graphics package used for Python for application development, interactive scripting, and publication-quality image generation across user interfaces and operating systems (Hunter, 2007); SciPy, open source scientific tools for Python (Jones et al., 2001-); NumPy, a structure for efficient numerical computation (van der Walt et al., 2011); Astropy, a community Python package for astronomy (Astropy Collaboration et al., 2013); APLpy, an open-source plotting package for Python hosted at http://aplpy.github.com; and the SIMBAD database, operated at CDS, Strasbourg, France (Wenger et al., 2000). 


\begin{abstract}
THE LOCAL CYGNUS COLD CLOUD AND FUTHER CONSTRAINTS ON A LOCAL HOT BUBBLE
\end{abstract}

Geoffrey Robert Lentner

July 20, 2015

Recent studies of the local interstellar medium have identified regions of nearby cold neutral gas with unexpected low temperatures and high pressures well within the boundaries of the local cavity (Meyer et al., 2006). Now, a multi-wavelength study of the local Leo cold cloud (LLCC) has strengthened our understanding of this apparent conflict with the conventional view of this environment (Peek et al., 2011, Meyer et al., 2012). The soft X-ray background observed here cannot be completely accounted for by a local hot bubble model (Snowden et al., 2015).

Interstellar absorption of $\mathrm{Na}$ I (D2 $\lambda 5889.591$ and D1 $\lambda 5895.924)$ was found towards $\delta$ Cygni (Welty et al., 1994). The present study will cover an extensive preliminary search of a $40 \mathrm{deg}^{2}$ field centered here. Using archival data from ELODIE, 41 of 284 targets have measurable interstellar absorption. Three of these occur along site lines to stars within the local cavity and represent the identification of a new nearby cloud.

Further, the author has developed a suite of precision software tools packaged as an open source library for Python 3. This library builds on other popular modern packages for Python in astronomy and may help to accelerate the pace of investigation and discovery in spectroscopy. 


\section{TABLE OF CONTENTS}

Page

DEDICATION

iii

ACKNOWLEDGEMENTS

iv

ABSTRACT

vii

LIST OF TABLES

xii

LIST OF FIGURES

xiv

\section{CHAPTER}

1 INTRODUCTION 1

1.1 The Interstellar Medium _ . . . . . . . . . . . . . . . . 1

1.1.1 Background .................... 1

1.1.2 The Local Bubble . . . . . . . . . . . . . . . 2

1.1.3 Searching for Interstellar Clouds . . . . . . . . . . . . . 4

1.2 Thesis Overview ...................... 4

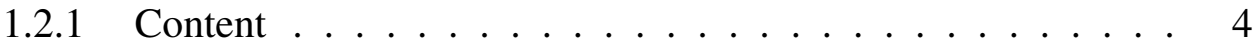

1.2.2 SLiPy ....................... 5

2 DATA $\quad 8$

2.1 Archival Data ................... 8

2.2 The Elodie Spectrograph . . . . . . . . . . . . . . . 8

2.3 Acquisition Methods . . . . . . . . . . . . . . . . . . . . 9

2.4 Sorting and Data Management . . . . . . . . . . . . . . . 13

2.4.1 The Simbad Database . . . . . . . . . . . . 13 
2.4.2 Managing FITS Files . . . . . . . . . . . . . 16

2.4.3 The Spectrum Datatype . . . . . . . . . . . . . . . . 17

3 CALIBRATIONS 22

3.1 Removal of Telluric Features . . . . . . . . . . . . . . . . . 22

3.1 .1 Description . . . . . . . . . . . . . 22

3.1 .2 Implementation . . . . . . . . . . . . . . . . . 24

3.2 Heliocentric Velocity Corrections _ . . . . . . . . . . 28

3.2 .1 Description . . . . . . . . . . . . . . . 28

3.2 .2 Implementation . . . . . . . . . . . . . . . . . . 29

4 MEASUREMENTS 31

4.1 Non-parametric Gaussian Kernel Regression _. . . . . . . . . . 31

$4.2 \quad$ Spectral Plots . . . . . . . . . . . . . . . . 32

4.3 An Interactive Graphical User Interface . . . . . . . . . . . . 34

4.3.1 The Voigt Profile . . . . . . . . . . . . . . 36

4.3 .2 Atomic Ions . . . . . . . . . . . . . . . . . . . . 37

4.3.3 Fitting Absorption Line Parameters _ . . . . . . . . . . . 39

4.3 .4 Equivalent Widths . . . . . . . . . . . . . . 41

4.3.5 Column Densities and the Apparent Optical Depth Method . . 46

4.4 Uncertainty Calculations . . . . . . . . . . . . . . . . . . . . . . . 49

5 RESULTS 51

6 DISCUSSION 66

6.1 Structure and Target Selection Bias $\ldots \ldots \ldots 6$

6.2 The Soft X-ray Background . . . . . . . . . . . . . 67

6.3 Stellar vs Interstellar . . . . . . . . . . . . . . . . 67 
6.4 Future Studies . . . . . . . . . . . . . . . . . . . . 68

7 CONCLUSIONS $\quad 71$

$\begin{array}{ll}\text { REFERENCES } & 74\end{array}$

$\begin{array}{ll}\text { APPENDICES } & 78\end{array}$

A HUBBLE SPACE TELESCOPE PROPOSAL 78

$\begin{array}{ll}\text { B OBSERVATIONAL METADATA } & 89\end{array}$

$\begin{array}{lll}\text { C } & \text { SOURCE CODE } & 101\end{array}$

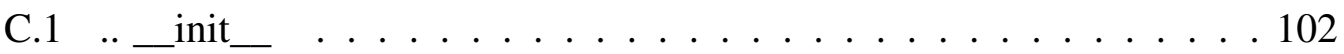

C.2 .. Algorithms . Functions . . . . . . . . . . . . . . . 103

C.3 .. Algorithms . KernelFit _ . . . . . . . . . . . . . . . 105

C.4 .. Data. Atomic . . . . . . . . . . . . . . . . . . . 107

C.5 .. Data. Elodie . . . . . . . . . . . . . . . . . . 111

C.6 .. Framework . Argument . . . . . . . . . . . . . . . 113

C.7 .. Framework . Command . . . . . . . . . . . . . . . . . . 114

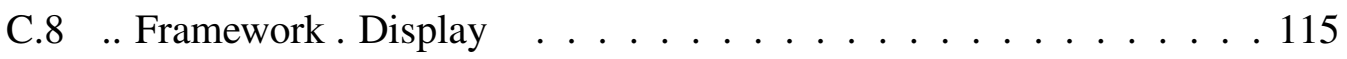

C.9 .. Framework . Measurement _ . . . . . . . . . . . . . . . 117

C.10 .. Framework . Options _ . . . . . . . . . . . . . . 118

C.11 .. SLiPy . Correlate . . . . . . . . . . . . . . . . . . . . 119

C.12 .. SLiPy . Fits _ . . . . . . . . . . . . . . . . . 120

C.13 .. SLiPy . Montage . . . . . . . . . . . . . . . . . . . 125

C.14 .. SLiPy . Observatory . . . . . . . . . . . . . . . . . 136

C.15 .. SLiPy . Plot . . . . . . . . . . . . . . . . . . . . . 146

C.16 .. SLiPy . Profile . . . . . . . . . . . . . . . . . . . 151

C.17 .. SLiPy . Simbad . . . . . . . . . . . . . . . . . . . . . 164 
C.18 .. SLiPy . Telluric . . . . . . . . . . . . . . . . . . . 168

C.19 .. SLiPy . Velocity . . . . . . . . . . . . . . . . . . . . 170

CURRICULUM VITAE

173 


\section{LIST OF TABLES}

TABLE $\quad$ Page

5.1 Non-OB Targets with Measurable Absorption - A . . . . . . . . . . . 55

5.2 Non-OB Targets with Measurable Absorption - B . . . . . . . . . 56

5.3 Non-OB Targets with No Absorption $-\mathrm{A} \ldots \ldots . \ldots 57$

5.4 Non-OB Targets with No Absorption - B . . . . . . . . . . . 58

5.5 Non-OB Targets Inconclusive $-\mathrm{A} \ldots \ldots$. . . . . . . . . . 59

5.6 Non-OB Targets Inconclusive $-\mathrm{B} \ldots \ldots$. . . . . . . . . . 60

5.7 Non-OB Targets Inconclusive $-\mathrm{C} \ldots \ldots$. . . . . . . . . . 61

5.8 Non-OB Targets Inconclusive $-\mathrm{D} \ldots \ldots$. . . . . . . . . . . 62

5.9 Non-OB Targets Inconclusive $-\mathrm{E} \ldots \ldots$. . . . . . . . . 63

5.10 OB Targets $-\mathrm{A} \ldots \ldots \ldots \ldots$

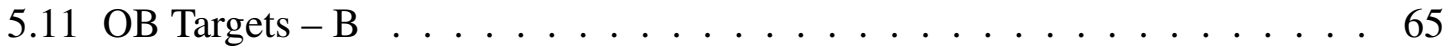

6.1 Stars Observed in the Region of $\delta$ Cygnus using the Coudè Feed . . . . . . 70

B.1 Observational Metadata for Non-OB Measurable Targets - A . . . . . . . . 90

B.2 Observational Metadata for Non-OB Measurable Targets - B . . . . . . . . 91

B.3 Observational Metadata for Non-OB No Absorption Targets - A . . . . . 92

B.4 Observational Metadata for Non-OB No Absorption Targets - B . . . . . . 93

B.5 Observational Metadata for Non-OB Inconclusive Targets - A . . . . . . . 94

B.6 Observational Metadata for Non-OB Inconclusive Targets - B . . . . . . 95

B.7 Observational Metadata for Non-OB Inconclusive Targets - C . . . . . . 96

B.8 Observational Metadata for Non-OB Inconclusive Targets - D . . . . . . . 97 
B.9 Observational Metadata for Non-OB Inconclusive Targets - E . . . . . . 98

B.10 Observational Metadata for OB Targets - A . . . . . . . . . . . . . 99

B.11 Observational Metadata for OB Targets $-\mathrm{B} \ldots \ldots$. . . . . . . 100 


\section{LIST OF FIGURES}

FIGURE $\quad$ Page

3.1 Telluric Correction - HD $192640 \ldots \ldots$. . . . . . . . . . . 26

3.2 Telluric Correction - Altair . . . . . . . . . . . . . . . 27

4.1 KernelFit1D Example . . . . . . . . . . . . . . . . 33

4.2 Example SPlot of Regulus . . . . . . . . . . . . . . . 35

4.3 SPlot of HD 192639 . . . . . . . . . . . . . . . . 42

4.4 SPlot of HD 192639 - Profile.MultiFit() A . . . . . . . . . . . . 43

4.5 SPlot of HD 192639 - Profile.MultiFit() B . . . . . . . . . . . . . 44

4.6 SPlot of HD 192639 - Profile.MultiFit() C . . . . . . . . . . . 45

5.1 Cygnus Field - All Data . . . . . . . . . . . . . . . . 52

5.2 Cygnus Field - Absorption . . . . . . . . . . . . 53

5.3 Cygnus Field - No Absorption . . . . . . . . . . . . . 54

6.1 Stellar vs ISM Lines . . . . . . . . . . . . . . . . . . . . 69 


\section{CHAPTER 1}

\section{INTRODUCTION}

\subsection{The Interstellar Medium}

\subsubsection{Background}

Before embarking on a study of a particular constituent of the local interstellar medium and our search for nearby cold clouds, it is instructional to begin with a brief description of what exactly the interstellar medium (ISM) refers to. Informative texts include Draine (2011), ${ }^{1}$ Spitzer (1978), ${ }^{2}$ and the earlier Aller et al. (1968). ${ }^{3}$

Generally, we can regard essentially all matter and interactions in the galaxy not of the stars as being a constituent of the ISM, or at least contributing to it, though a more limited grouping is more useful. The Milky Way galaxy is approximately 100,000 light years (30.7 kiloparsecs) in diameter and home to on the order of a few hundred billion stars. However much mass is contained within all the stars in the galaxy, there is comparable mass in the diffuse gas between them. On average, there is approximately one atom/molecule per cubic centimeter in the ISM. This is in fact a better vacuum than most modern laboratories achieve. And yet, across the vast distances of interstellar space, this material together forms intricate structure on both small and large scales.

Both the composition and dynamics in the ISM are the subject of research, old and new. We know that the ISM is mostly hydrogen and helium (in proportions we expect

\footnotetext{
${ }^{1}$ Physics of the Interstellar and Intergalactic Medium

${ }^{2}$ Physical Processes in the Interstellar Medium

${ }^{3}$ Nebula and Interstellar Matter, Stars and Stellar Systems, Vol VII
} 
from knowledge of the stars and Big Bang nucleosynthesis), more or less 3/4 and 1/4 respectively. The hydrogen in our galaxy can be broken into two major components. The first of these is referred to as HII regions (though this notation is somewhat unique to astronomy) and is composed of hot, photoionized $\left(\mathrm{H}^{+}\right)$hydrogen that surrounds young $\mathrm{O}$ and B type stars and is also distributed in the disk and halo. This gas is even more diffuse than described previously and lives at temperatures on the order of millions of kelvin. In contrast, large portions of the galaxy contain regions of hydrogen gas referred to as HI. This is cold $(\sim 10 \mathrm{~K})$ neutral hydrogen. These regions trace much of the structure of our galaxy and constitute a considerable fraction of the mass of the ISM. This gas can emit a signature $21-\mathrm{cm}$ line from hyperfine atomic transitions. There is so much that could be said about the rich physics played out across the galaxy - emission, absorption, extinction, gravitation, magnetic fields, electrostatics, star formation; a complete survey would include a substantial fraction of modern astrophysics.

One important component within the ISM though is the abundance and distribution of metals, the elements heavier than helium $(\mathrm{z}>2)$. These element paint a picture of the history and evolution of our galaxy. The first stars contained precious little heavier elements, relatively speaking, and in their death polluted the ISM with their enriched material. Every generation of stars that has followed continued this cycle of enrichment. These heavier elements offer tracers of particular physics. We can use absorption studies to not only understand the chemical evolution of our galaxy but to learn the local structure and dynamics in the immediate vicinity of the Sun.

\subsubsection{The Local Bubble}

The Local Bubble refers to a relatively evacuated region (or void) immediately surrounding the Solar System. Within the past 25-60 million years an expanding wave from supernovae explosions blew out an already low density region created by massive 
stellar winds and displaced the local ISM to create large magnetized bubbles hundreds of parsecs across (Frisch, 2007). The boundaries of this cavity have been traced using several techniques including color excess, HI radio emission, etc. Generally, this void is thought to be filled with hot, diffuse, X-ray emitting gas at around $10^{6} \mathrm{~K}$ (Snowden et al., 1998). Cool, more dense molecular clouds dot the boundaries and have been compressed to create star forming regions.

Our understanding of this local environment has taken considerable shape over the past few decades (and even just in the past few years). Recently, we have found that there are several warm $\left(\mathrm{T} \sim 10^{4} \mathrm{~K}\right)$ clouds inside this cavity; and even that the Solar System itself lives in one of these warm clouds (Lallement et al., 2003). It was generally believed that there was no colder interstellar material in this Local Hot Bubble (LHB); however, one cloud originally identified by Verschuur (1969), has presented a challenge to this view for many years. Meyer et al. (2006) used observations of Na I (D2 $\lambda 5889.591$ and D1 $\lambda 5895.924)$ and found cold interstellar absorption towards 23 stars behind the cloud. The distance to the closest of these stars ( $\sim 42$ parsecs) places this local Leo cold cloud (LLCC) well within the boundaries of the local cavity. Peek et al. (2011), using optical observations of the LLCC, constrains the distance to this material between 11 and 24.5 parsecs. Meyer et al. (2012) used observations from the Hubble Space Telescope of interstellar C I to directly measure the pressure in the cloud. The $40,000-80,000 \mathrm{~cm}^{3} \mathrm{~K}$ thermal pressure found is much greater than was expected and indicates that it is not in thermal pressure equilibrium with its surrounding environment. Further, analysis of Rosat All Sky-Survey 1/4 keV (C-band) data (Peek et al., 2011, Snowden et al., 2015) shows only weak shadowing of X-ray flux towards the LLCC, indicating that a LHB of diffuse X-ray emitting gas cannot be the only source of the soft X-ray background radiation (SXBR). 


\subsubsection{Searching for Interstellar Clouds}

It is difficult to make generalizations about the consequences and constraints placed on a LHB using only a single object. It is important to search for other, possibly similar, cold clouds in the vicinity of the Sun to further strengthen any conclusions drawn here. To this end, we are interested in conduct such a search. Recently, another field of astronomy has enjoyed much success and popularity - the search for extrasolar planets. Several new archives of moderate resolution spectra have become available and public access. These archives offer an opportunity for science outside the domain of radial velocity studies. This work consists of one such preliminary search for a nearby cold cloud.

Welty et al. (1994) has shown absorption of interstellar sodium towards $\delta$ Cygni. The hypothesis is now that there exists another of these cold neutral clouds in the direction of Cygnus. To investigate the possibility of placing further constraints on a Local Hot Bubble, an extensive search of a 40 degree square field centered here consisting of 284 spectra from the ELODIE archive has yielded numerous targets for which absorption is present; the closest of these is HD 184006 at $\sim 37$ parsecs.

\subsection{Thesis Overview}

\subsubsection{Content}

Each major component of this study is discussed in the following chapters, largely in procedural order. All the data used here was retrieved from the ELODIE archive. In Chapter 2, I discuss the exhaustive procedures necessary to acquire all relevant spectra to the present work. Every spectrum needed to be calibrated, including the removal of telluric absorption lines and performing heliocentric velocity corrections. These steps in and of themselves (considering the quantity of data) in addition to some measure of quality assurance were essentially the motivation for creating a new library of precision 
software tools in a modern language and style (to be discussed). I cover these steps in Chapter 3. Further, the actual practice of fitting absorption profiles and extracting accurate measurements with appropriate uncertainties necessitated the development of an interacting, flexible fitting routine. The details of this routine as well as numerical and visual representations of the results of this work are provided in Chapters 4 and 5, respectively. In Chapter 6, I discuss the relevance of these results to the Local Hot Bubble model and our understanding of the soft X-ray background (SXRB). There is still much work to do in this area, specifically as it relates to the local Cygnus cold cloud (hereafter LCCC); A recent Hubble Space Telescope proposal is attached in Appendix A (the author is a co-investigator on the proposal). Further, I am publishing all the relevant metadata for all of the observations used in this study. This includes the file indices for accessing the data from the archive as well as the date and time of observation, the exposure time, and the signal-to-noise ratio. This metadata is provided in Appendix B in Tables B.1 - B.11. The tables in Chapter 5 and Appendix B that correspond to targets with measured interstellar $\mathrm{Na}$ I absorption are annotated with index numbers that label markers in Figures 5.2 and 5.3. Last, in Appendix $\mathrm{C}$ the full source code for the entire library is included for completion (with a few exceptions). This code is up-to-date as of August 10, 2015. Though, most of the code is provided, some auxiliary components have been neglected for reasons of practicality.

\subsubsection{SLiPy}

We are interested in continuing to identify other cold clouds; therefore, the ELODIE archive has been parsed and retrieved for every unique stellar target - one file with the highest signal to noise. This constitutes 5, 296 targets (if we restrict ourselves to only HD, HR, GC, GJ, and BD catalogues). There are 284 targets within $20^{\circ}$ of $\delta$ Cygni (the field of interest). With this many data elements, it is import to not only be consistent, 
but to be efficient. Modern astronomy is headed in the direction of so called Big Data and it is becoming more critical for astronomers to develop new technical and software skills and strategies to meet this demand. Here, this quantity of spectra is at the limit for what is possible to analyze iteratively and with consistent quality. SLiPy (the Spectroscopy and astrophysics Library for Python 3) was created to accelerate my work in managing, analyzing, visualizing, and extracting measurements from spectra. It is open source (under the GNU General Public License, version 3) and is free to download from its website.

The focus of this research has been on the science; however, the reality is that the bulk of the work done here has been related to data management. We not only need to do good science but we need to do it efficiently and consistently. One of the core components of science is reproducibility. The idea behind creating much of this software has been that if it is at all possible to automate a particular procedure, the quality and efficiency of the analysis increases. Further, later work can much more easily confirm or reject these and other results with confidence because the process for making or failing to make a detection is reproducible.

In an effort to simultaneously lay bear a census of my research and document the syntax of using this library (SLiPy) I will provide code snippets along the way. The alternative would be to provide another appendix of documentation and usage details that would be referenced throughout. In-text examples however are more easily comprehensible. I will necessarily assume that the reader is familiar with the Python language to the extent that basic concepts will not be explained here. In keeping with the syntax style of packages/modules/functions/methods in Python, all of these will be referenced using bold face type. For example, Algorithms.Functions.Lorentzian() is referencing the Lorentzian function inside the Functions module contained within the Algorithms sub-package of the SLiPy package. Leading punctuation will be used to indicate a function that is a method for a class (e.g., .resample() is a method of the 
Spectrum class). Also, the name of explicit arguments to functions will be given via italics. For example, filepath and keyword are arguments to the Fits.Header() function. Last, the snippets will at times compound sequentially. That is, previous variables and imports will not be reproduced in every snippet. Further, the representation of the result on the final line of the snippet may be printed immediately below it. For example:

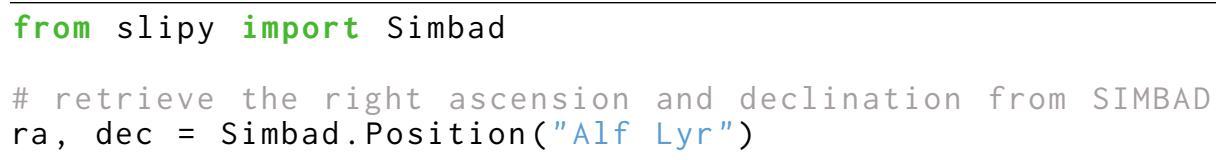

In the above code snippet, the numerical output below the horizontal line is the result of issuing $[\mathrm{ra}, \mathrm{dec}]$ at the interpreter. 


\section{CHAPTER 2}

\section{DATA}

\subsection{Archival Data}

The recent growth in the study and search for extrasolar planets has produced several spectral archives, much of which is public access (i.e., no longer proprietary). These archives offer a number of opportunities to do good science outside the domain of extrasolar planets. This study is one such opportunity. We have an interest in searching both the Keck HIRES (Vogt et al., 1994) and ELODIE (Baranne et al., 1996) archives for absorption features indicative of local interstellar clouds. The present study principally relies on the ELODIE archive, as the data products offered are already reduced and ready for investigation. The following sections outline the use of this archive. Future work will include the automated reduction and investigation of the Keck HIRES data.

\subsection{The Elodie Spectrograph}

This study is based on data retrieved from the ELODIE archive. ELODIE is a fibre-fed, cross-dispersed echelle spectrograph that was mounted ${ }^{1}$ at the 1.93 meter telescope at the Observatoire de Haute-Provence, France (Baranne et al., 1996, Moultaka et al., 2004, Prugniel et al., 2007). Its design purpose was to provide high accuracy radial velocity measurements (needed to search for brown-dwarfs and giant planets around

\footnotetext{
${ }^{1}$ The spectrograph was replaced by the SOPHIE (Spectrographe pour l'Observation des Phénomènes des Intérieurs stellaires et des Exoplanètes) echelle spectrograph at the 1.93 meter telescope in 2006 (Bouchy and Sophie Team, 2006).
} 
nearby stars) and has resolution $42000 .^{2}$

The ELODIE archive is an online repository of all the data taken by the spectrograph. The database contains 35,517 spectra. ${ }^{3}$ Users can access content via an intuitive web interface that allows searching for specific targets, by position, as well as some advanced search options. The search results provide a list of names of targets resolved by the archive with a preview option and a customization tool that allows for some pipeline processes before downloading. Among these is the ability to resample the spectrum (e.g., 5850-5950 $\AA$ at $0.01 \AA$ pixel $^{-1}$ ) and to perform continuum normalization. The high accuracy radial velocity spectra in addition to the already reduced data products with further pipeline options made ELODIE an attractive option for this research and was the reason the decision was made to rely on it.

\subsection{Acquisition Methods}

In addition to the web interface, the ELODIE archive can be accessed directly using url scripting. For example:

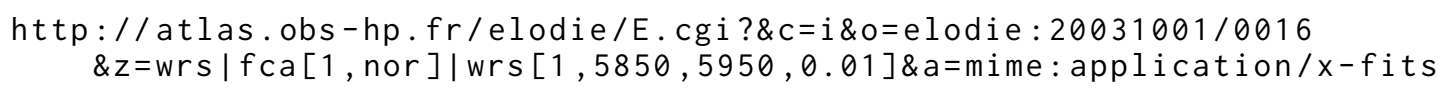

The above url has several components to it. First, 20031001/0016 is the index of a particular file in the archive (like those listed in Tables B.1 - B.11 in Appendix B). Second, the $z=w r s \mid f c a[1$, nor $] \mid w r s[1,5850,5950,0.01]$ segment is a pipeline command. fca[1, nor $]$ says to perform the continuum normalization. wrs $[1,5858,5950,0.01]$ is a wavelength resampling command; in this case we have requested to resample as in the example used previously from $5850-5950 \AA$ at $0.01 \AA$ pixel $^{-1}$.

\footnotetext{
${ }^{2}$ In astronomy, resolution is defined as $R=\lambda / \Delta \lambda$.

${ }^{3}$ Previously, the archive contained around 30, 600 spectra and only about half were public access. In the past few months however all spectra have been made public.
} 
Previous work has identified an interstellar Na I D1 component ${ }^{4}$ towards $\delta$ Cygni at a distance of $51 \mathrm{pc}$ within the edge of the Local Bubble (Lallement et al., 2003, Welty et al., 1994). As such, we were interested in searching a field centered here to identify whether there was a large interstellar cloud in this vicinity. A 20 degree radius (or more precisely, everything with a right ascension and declination within 20 degrees of $\delta$ Cygni) provided ample sampling of the area. The difficulty arises when trying to browse such extensive search results manually to decide which spectra are best and to only select one for a unique stellar target. To this end, the entire archive was accessed to the extent that by giving the positional search form the whole sky, every single file in the archive was returned in the results. This text was exported and used to construct an ascii catalogue of the entire archive containing a listing of all files. These files are organized according to the identifier they belong to. Information also listed is the signal-to-noise ratio for the spectrum reported by the archive. With this information we can use scripting techniques to comprehend the archive using any criteria of interested. How exactly this is done is covered in the next section.

After deciding what spectra (the indices of the spectra we want) to download, the actual process of downloading them has been incorporated into SLiPy. It is quite useful to have a piece of software be able to access data on your behalf in an intuitive way via a standardized API ${ }^{5}$. Within the SLiPy package, there is now a dedicated Data sub-package with a few functioning data management products. One of these is the Elodie module (see Appendix C.5 for the source). In the future, the goal is to incorporate many other modules for accessing data from astronomical archives.

The Elodie.Archive is an object that once instantiated reads in the raw ascii catalog mentioned previously (from ../Data/Archives/Elodie.csv by default) and parses the

\footnotetext{
${ }^{4} W_{\lambda}=20.2 \mathrm{~m} \AA, v_{\odot}=-18.54 \mathrm{~km} \mathrm{~s}^{-1}, \mathrm{~N}=29.610^{10} \mathrm{~cm}^{-2}, b=0.42 \mathrm{~km} \mathrm{~s}^{-1}$. (Welty et al., 1994)

${ }^{5}$ Wolfram's Mathematica (C) for example is a very sophisticated language that has built in data accessor methods.
} 
data into a set of two Python dictionaries, .files and .data. These are indexed by unique target names. The .data member has a list of pairs consisting of the name of the file and the signal to noise for that spectrum. .files contains the reduced archive and by default consists only of $\mathrm{HD}, \mathrm{BD}, \mathrm{HR}, \mathrm{GC}$, and GJ objects, ${ }^{6}$ choosing the file pertaining to the spectra with the highest signal-to-noise ratio available.

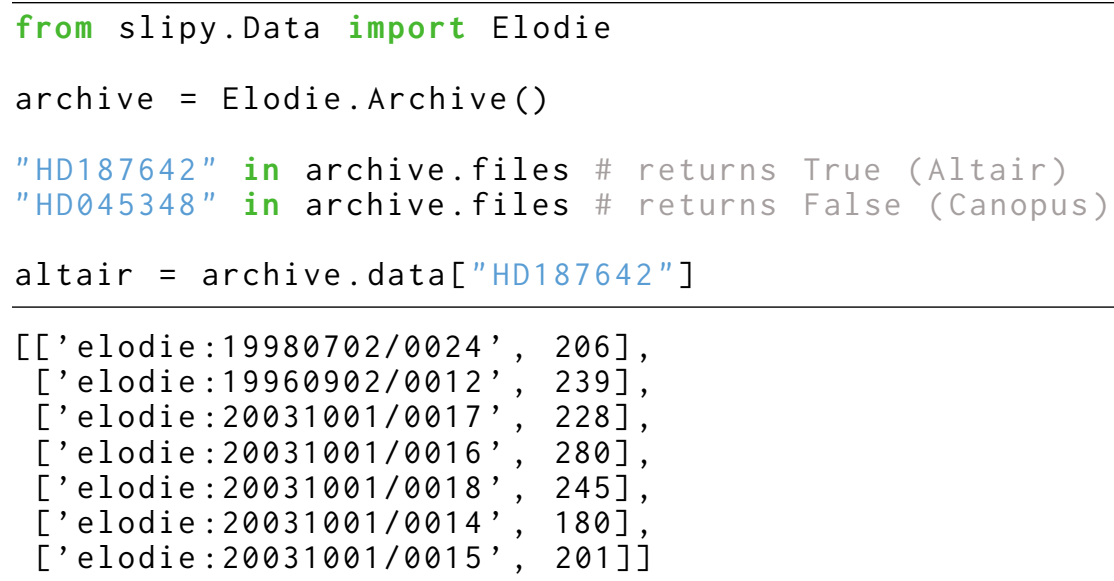

Also included within the module are the Elodie.Script() and Elodie.Download() functions. Elodie.Script() was not really intended as an end-user product, but is a convenient helper-function to Elodie.Download(). It merely takes the properly formatted file index of a spectrum from the archive (and optionally a pipeline command-string) and constructs the necessary url script to download that file to your machine.

Elodie.Download() is a high-level function that actually retrieves the file for you. The idea here is that in the future the SLiPy.Data sub-package will be expanded to provide access to numerous archives, surveys, and data sets - all with similar accessor methods. So while the content of the different archive members will invariably be distinct, the methods for retrieving that data from within the software will be uniform. For this study, the Elodie module allowed me to concisely crawl and sort the archive using Simbad (a module

\footnotetext{
${ }^{6}$ Henry Draper, Bonner Durchmusterung, Bright Star (though abbreviated using the name of its predecessor the Harvard Revised Photometry Catalogue), General Catalogue, and Gliese-Jahreiss (respectively) are all the names of stellar catalogues.
} 
discussed in the following section). Ultimately, I was able to automatically identify the 5, 296 unique stellar targets ${ }^{7}$ and retrieve the corresponding spectrum with the highest signal-to-noise ratio available. ${ }^{8}$

Elodie.Download() takes an arbitrary number of file names (in the format represented above) and actually downloads them to your machine for you. There are a few options as well. First, both the continuum normalization and wavelength resampling pipeline options are accessible. By default, normalize is set to True and resample is left unspecified (leaving the spectrum at the original wavelength calibration). To specify a desired resampling, pass a tuple of length three to resample (starting wavelength, ending wavelength, and resolution in angstroms and angstroms per pixel respectively). Further, by default the FITS files will be downloaded to your current working directory. To specify an alternative location, give the desired relative path as outpath. Also, all files downloaded will have names matching the pattern, "elodie-yyyymmdd-nnnn.fits". If you provide a list of file names equal in length to the number of files requested, the downloaded FITS files will be given these alternative names instead. Last, this can be a non-trival length of time needed to download a data set. By default verbose is set to True and a progress bar is displayed while the files are being downloaded and an estimated time of completion is given.

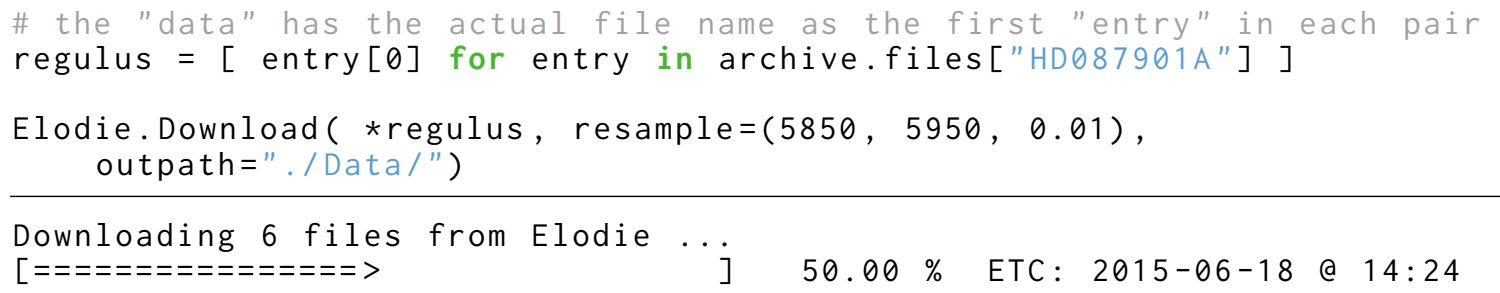

\footnotetext{
${ }^{7}$ Sometimes in the ELODIE archive there are stars which are the same but are labeled with an appended "A" or "B" (not necessarily corresponding to binary systems). As such, about a dozen of the targets from the 284 identified in the Cygnus field are actually duplicates. This has been handled appropriately.

${ }^{8}$ While not necessarily representing the absolute best piece of data, using the $\mathrm{S} / \mathrm{N}$ as a benchmark for automatically sorting the data quality is useful. In a few instances, despite having the highest $\mathrm{S} / \mathrm{N}$, spectra from the archive were of too poor quality to provide any meaningful results.
} 
In the above code snippet the *regulus syntax means to expand the list as if we had typed them in manually. Here, we would have downloaded the six files from the Elodie archive for Regulus ${ }^{9}$ to a "Data" directory within the current working directory.

\subsection{Sorting and Data Management}

As alluded to previously, there are a number of modules developed for SLiPy that provide tools for handling and managing astronomical data. Among these (to be discussed here) are the Simbad, Fits, and Spectrum modules.

\subsubsection{The Simbad Database}

The Simbad (see Appendix C.17) module allows the user to query the SIMBAD astronomical database from inside Python or shell commands and scripts. It's four current major functions Position, Distance, Sptype, and IDList return real variables with appropriate types ready for use. While the module's implementation was remarkably strait forward, its impact for this research was nearly unmatched in terms of what it afforded to me in my ability to quickly and confidently put data elements into context and organize them. A full $85 \%$ of the content provided in Tables $5.1-5.11$ and B.1 - B.11 were auto generated using these functions.

The approach taken is similar to the accessor method from the Elodie module. Here, a Simbad.Query class uses the Simbad.Script() and Simbad.URLEncode() helper functions to put together a url script recognized by the SIMBAD astronomical database. When querying the database, a file is returned with some standard retrieval information and statistics along with the results of your query. The format for this file is quite standardized and allowed for a common query object that is instantiated by each of

\footnotetext{
${ }^{9}$ Unfortunately, as described previously, some of the targets have an "A" appended to them. If you think a star is in the archive and it comes up false, try adding an " $\mathrm{A}$ ".
} 
the specialized functions. That is, the Simbad.Distance(), Simbad.Position(),

Simbad.Sptype(), and Simbad.IDList() functions are wrappers to creating the query

object. They each take a string value specifying the identifier of the target of interest.

Each function then creates the query object with a specialized parameter string ${ }^{10}$ specific to the attribute of interest and retrieves the file from SIMBAD. The final step is to extract the relavent information from the file and return the data with an appropriate type (either a string or a Quantity $\left.{ }^{11}\right)$.

The following are some example uses. The format of the identifier string is very flexible and can be literally anything that would otherwise be recognized by conducting a search by identifier using the online search form at simbad.u-strasbg. $\mathrm{fr} / \mathrm{simbad}$.

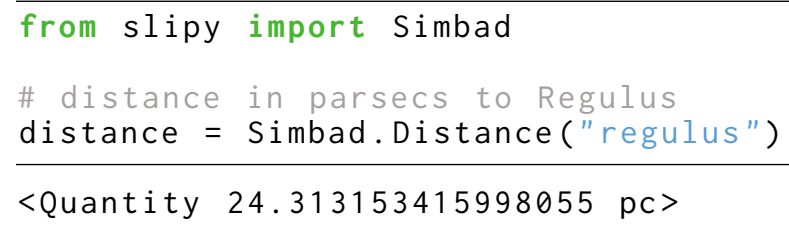

\footnotetext{
${ }^{10}$ For example, $\% \mathrm{COO}(\mathrm{d} ; \mathrm{c})$ is the code for retrieving the right ascension and declination of the target in decimal degrees.

${ }^{11}$ The Quantity object is meant to represent a value that has some unit associated with the number. (Astropy Collaboration et al., 2013)
} 


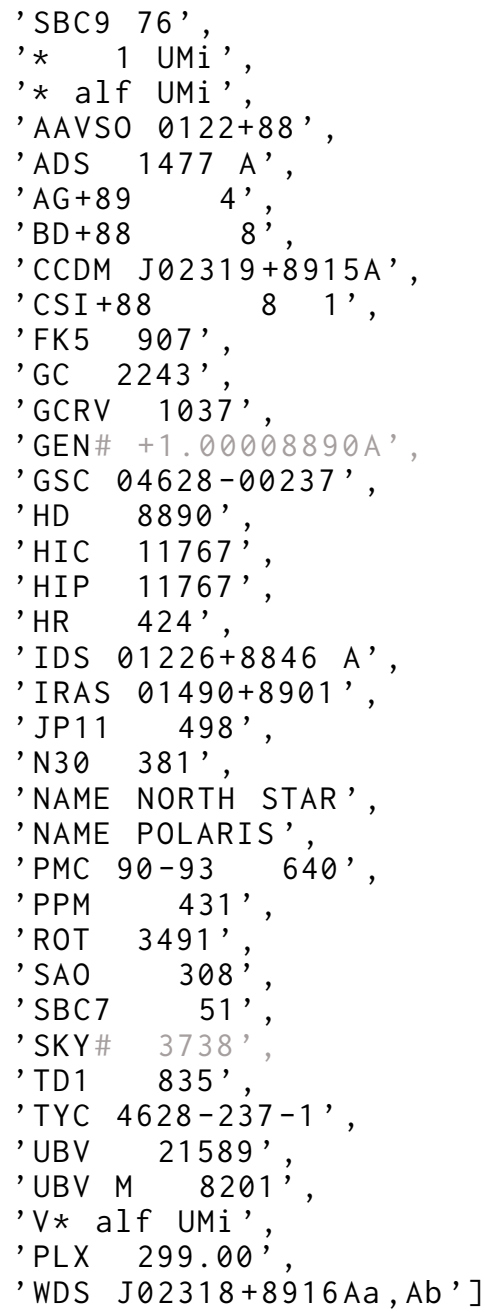

Taking the names of the available unique targets from the ELODIE archive and doing a search for their right ascension and declination I was able to restrict my list to site lines inside the $40^{\circ}$ field of interest. Before downloading the files, I was able to again query SIMBAD on this smaller set of targets and retrieve their spectral types. Inserting the first letter of the spectral type into the path of the names given to Elodie.Download() (e.g., "/A/”) after creating the appropriate directory structure, I was able to easily organize the data by spectral type. 


\subsubsection{Managing FITS Files}

In order to analyze these data we need to get it out of the FITS files. Many software libraries and languages have implementations for doing such. In fact, within Python the Astropy Project ${ }^{12}$ already has significant capabilities (Astropy Collaboration et al., 2013). The Fits module within SLiPy builds on the functionality provided by astropy.io.fits. The most significant item provided by the module is the Fits.GetData() function. I'll document its usage here, and in relation to it the usage of some other related functions.

Fits.GetData() can be used in two ways. First, an arbitrary number of files can be requested via their relative paths. Second (or in combination with the first), you can provide the name of a toplevel directory under which there exists some FITS files. With the first method it will simply extract the data from those listed files. With the second, either the Fits.Find() or the Fits.RFind() function will be used first to search under or recursively under the toplevel directory named. By default it uses Fits.Find() but if recursive is assigned True then it uses Fits.RFind(). Both Fits.Find() and Fits.RFind() take two optional arguments. If no arguments are given it will return a list of the relative paths to *.fits files below the current working directory. If given an argument, it is expected to be the relative path to an alternative directory (under which it will search for *.fits files). The second argument, when provided, is an alternative glob pattern to match (e.g., *.fits.gz).

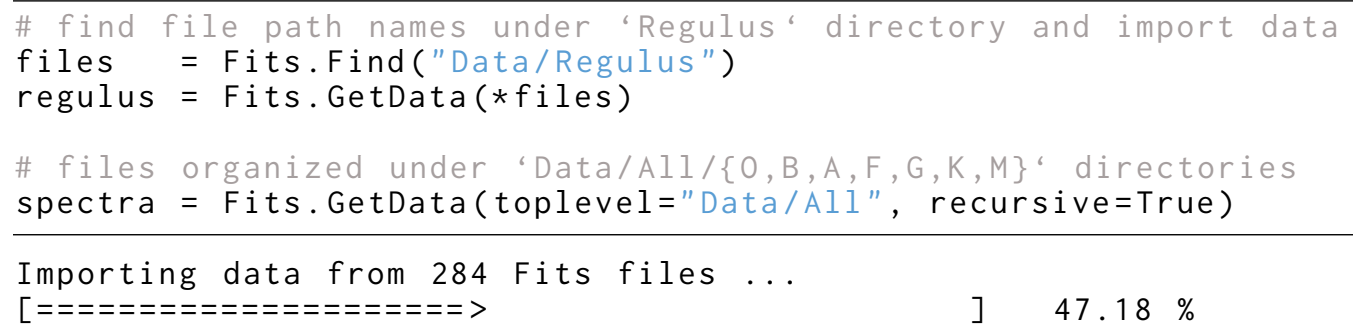

${ }^{12}$ The Astropy Project is a community effort to develop a single core package for Astronomy in Python and foster interoperability between Python astronomy packages (www.astropy.org). 
In the above snippet, we have imported two groups of spectra. First, the six files for Regulus from the previous example are now assumed to be under the "Data/Regulus" directory. Their paths are read in and expanded in the files arguments to the Fits.GetData() function. Similarly for the remainder of the FITS files, except they are housed under subdirectories according to spectral type. The return type of this function is a Python list of Spectrum objects.

\subsubsection{The Spectrum Datatype}

In an effort to reduce complexity and make operations more intuitive, a new data type was created, the Spectrum. As of today, I argue that there does not currently exist an implementation of a 1D spectrum object for the Python language as functional and dynamic as this one. It is important to highlight this aspect of SLiPy because throughout the following chapters I will necessarily refer to the arguments of the various functions as being of type Spectrum.

A Spectrum can be created two distinct ways. The first (as done by the Fits.GetData() function) is to provide a filename. With a proper FITS file containing both the flux data and also the corresponding wavelength calibration, the Fits.Header() function is used to retrieve the "CRPIX1", "CRVAL1", and "CDELT1" values from the header and construct a wavelength array equal in length to that of the flux data. ${ }^{13}$ Once created, the flux data and wavelength array can be accessed independently with the .data and .wave members respectively. Units are bound to the arrays via astropy.units. ${ }^{14}$ If the units are not specified by the xunits and yunits keyword arguments then the flux data (yunits) will be dimensionless and the wavelength array (xunits) will be in Angstroms. If

\footnotetext{
${ }^{13}$ Although you can request an alternative key to be read from the FITS files by specifying it as an argument of the same name (e.g., Spectrum("myData.fits", crpix1="something else")).

${ }^{14}$ While units are bound to the arrays, it is assumed unless provided by the xunits and yunits keywords that they are dimensionless.
} 
defined on 550-600 $\mathrm{nm}$, the operation Spectrum $=$ SpectrumA + SpectrumB would yield a new spectrum with the wavelength domain of SpectrumA where all pixels that fall into the domain of SpectrumB are operated on. The necessary condition is that the RHS spectrum is equivalent to or entirely contained (inclusively) by the LHS.

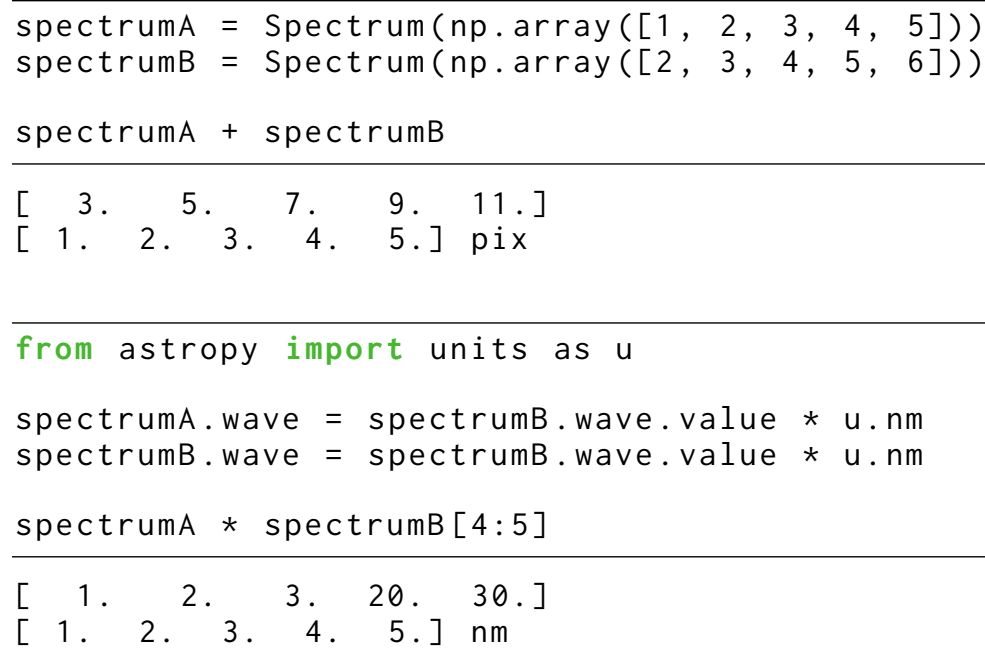

In the immediately above code snippet, the wavelength arrays are given units of $\mathrm{nm}$ because you cannot operate on two spectra that have pixel units for wavelength arrays unless they are the same length (the alternative makes no sense!). Only the last two elements were operated on. The [4:5] style notation is called slicing and is common in several other programming languages in addition to Python. Here, its been implemented in a non-standard way. Despite how it may appear, the command does not say to select elements 4-5, but instead says to retrieve the spectrum defined between those wavelength values (assuming the same units as the wavelength array). The spectra used in this study are defined on 5850-5950 A. So regulus [5885:5910] would return a new spectrum as a segment of the Regulus spectrum, all pixels contained within those two wavelength values. Additionally, a third component to the slice may optionally be given to specify a desired resolution. regulus[5885:5910:0.1] says to take the same segment but resample onto a new resolution (the current being $0.01 \AA$ pixel $^{-1}$ ). This is simply syntactic sugar and is 
equivalent to the .resample $\left(\right.$ ) function ${ }^{16}$ (e.g., regulus. resample $(5885 *$ u. AA, 5910 * u. AA, 250), where the third argument is now the number of pixels not the resolution). Spectra can also be operated on by scalars (left hand and right hand operations).

$47 \quad 2 *$ spectrumA

$\left[\begin{array}{llllll}{[} & 2 . & 4 . & 6 . & 8 . & 10 .]\end{array}\right.$

The comparison operators return a Spectrum object but with binary, dimensionless data. In the case where the LHS and RHS don't have identical wavelength domains, the above described behavior holds, with the exception that all pixels in the LHS spectrum that remain unaffected by the domain of the RHS are returned as false (i.e., "0"). This can be interesting and useful functionality to have. ${ }^{17}$

The shift operators $(<<,>>)$ do what you might expect. They subtract from or add to (respectively) the .wave array (as oppose to the .data array for the other operators).

spectrumA $>2 * u \cdot n m$

[ 1.2 .3 .4 .5$.

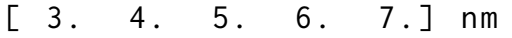

One could then, for example, manually apply a velocity correction as follows.

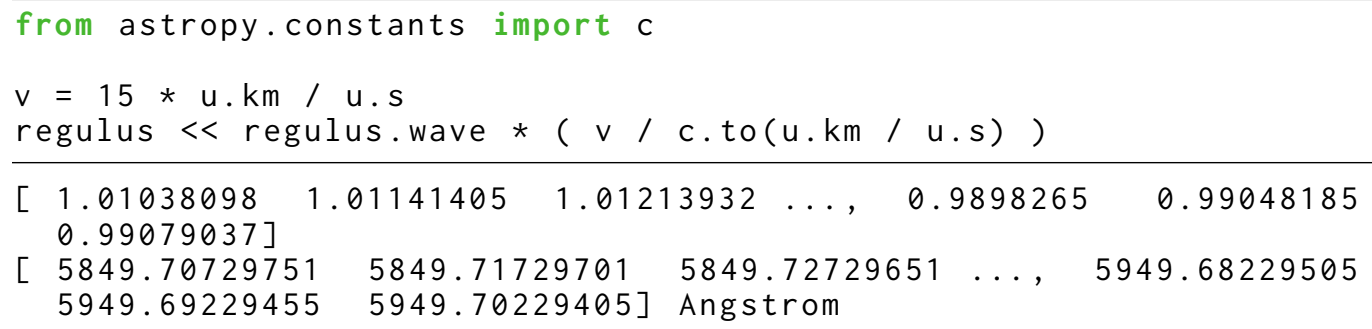

There are several other areas of functionality provided by the SLiPy.Data sub-package, the Fits module, and the Spectrum object; descriptions of these features are resigned to the online documentation.

\footnotetext{
${ }^{16}$ The .resample() method for a spectrum performs linear interpolation by default but can employ any other method of interpolation offered by the backend function, scipy.interpolate.interp1d(), by passing the kind keyword argument (e.g., kind = "cubic").

${ }^{17}$ For example, sum ( (regulus $>1$ ). data ) would tell you how many pixels have flux data greater than one.
} 
With these tools, along with the functions and algorithms described in the following chapters, one can more easily process larger spectral data sets with consistency. 


\section{CHAPTER 3}

\section{CALIBRATIONS}

\subsection{Removal of Telluric Features}

\subsubsection{Description}

Generally speaking, spectral data of the sort used in this study will have three categories of absorption line features. First, the star (whence the photons arise) is both a source and an absorber of photons. The stellar lines will likely be the largest (greatest equivalent width). Second, one or more interstellar sources of gas and dust along the site line between the star and Earth can and will add more features to the spectrum (often blending with stellar features). Last, as the photons propagate through our atmosphere, specific wavelengths will be absorbed from the atomic and molecular species present therein.

One of the first pressing issues to deal with given ground based observatories is the presence of numerous absorption features in the data that arise from the Earth's atmosphere, otherwise referred to as telluric absorption. A popular approach taken to address this issue is to remove these lines by dividing them out using a reference or calibation spectrum. The idea is that by taking the spectrum of a star, ideally before and after your science target, for which there are no stellar/interstellar absorption features in the domain of interest, one can preferentially select out the features that are telluric in origin. ${ }^{1}$ That is to say, if something is present in both spectra, by dividing one spectrum

\footnotetext{
${ }^{1}$ The reference star of choice will be a spectral type "A" star, though sometimes a "B" star is possible.
} 
by the other, the offending line can be removed. An import recognition here is that this only applies for spectra that have been continuum normalized.

Algorithmically, where there is no absorption line in the spectrum the continuum will ideally be very close if not exactly equal to 1 . As such, dividing by unity proffers no change. The absorption features come down from the continuum. So in order for the division operation to work, the spectrum needs to be inverted - with the continuum at zero and signal rising above it. So we flip both spectra, perform the division, then again invert the spectrum.

Practically speaking, this is not always a clean procedure. If the two spectra are taken during significantly different times or along differing lines of site through the atmosphere (differing airmasses), the telluric features can and will have conflicting shape and saturations. The relative strength between two corresponding lines both within the same spectrum and between spectra can cause inappropriate artifacts in the resulting corrected spectrum. For this study, there were numerous stellar targets from within the ELODIE archive of early type stars from which to choose. The difficulty is in choosing the right calibration star such that there are also no interstellar features.

A complete treatment of the options and benefits of various procedures for dealing with telluric features could occupy a lengthy paper, if not several. Here, I wish to demonstrate the successful implementation of the chosen approach and comment on its effectiveness and its drawbacks. For our purposes, Regulus ( $\alpha$ Leo, HD 87901), a B8IV star, was the best choice. While the time of observation and the position do not necessarily line up well with the targets of interest in this study, it has been demonstrated to high order that Regulus lacks an interstellar Na I D1, D2 component ${ }^{2}$ (Peek et al., 2011), an arguably more critical requirement. Because of the differing airmass (extinction levels), time of

These stars make good reference stars because they have little to no metal absorption features. That is to say they have little or no features other than broad Hydrogen lines in our region of interest.

${ }^{2}$ The lower limit for an interstellar component is $0.1 \mathrm{~m} \AA$. 
observation, and exposure between the Regulus spectra and that of the target spectra, it was necessary to modulate any spectral division algorithm to prefer calibrations that better fit the data. In other words, because the telluric features present in a spectrum of Regulus and that of a particular target could potential differ significantly, we needed to incorporate an auto-correcting feature to the algorithm that would preferentially choose a spectrum of Regulus out of many that best fit the current spectrum and choose that calibration.

\subsubsection{Implementation}

The Telluric.Correct() function (see Appendix C.18) takes two or more Spectrum arguments. The first is the spectrum to be corrected. The second argument, and any further arguments are to be candidate reference spectra. In this particular function, it is necessary to insure that the target spectrum occupies the same domain (not necessarily the same resolution) as the calibration spectra. One of the hallmarks of a telluric line is that it will be at zero velocity, or at least in principle it will be very near zero. As such, no velocity shift needs to be applied to the spectra before division. However, often there will be a wavelength difference of a few pixels (the extent of which depends on the resolution of the spectra). For this reason, a horizontal cross-correlation is performed to determine the optimal shift in pixels left or right on the calibration spectrum using the Correlate.XCorr() function (see Appendix C.11). Further, because of differing exposure times, etc., we necessarily must amplify the calibration spectra to best fit one with the other. Our goal is only to trace the continuum and telluric lines. The best fit from both of these correlations is quantified by minimizing the RMS (root mean square) difference between the spectra. This procedure is applied to all the provided candidate calibration spectra and the best fit is chosen to use for the division.

Options for this function include the lag for the wavelength correlation. This argument by default is 25 pixels but can be overridden; it is passed to Correlate.XCorr() 
and specifies how many pixels to shift the two spectra apart in searching for the best fit. Also, a range can be specified for amplitude correlation. This should take the form of a tuple of length three and is the start, end, and number of percentages (e.g., range $=(0.5,2.0,151)$ is the default and specifies a search between $50 \%$ and $200 \%$ of the calibration spectrum on a $1 \%$ increment). It's important to note here that only on the part of the spectra that overlap is the operation applied; so if the best shift of the chosen calibration spectrum is three pixels, than that many pixels on either side of the target spectrum will not be altered.

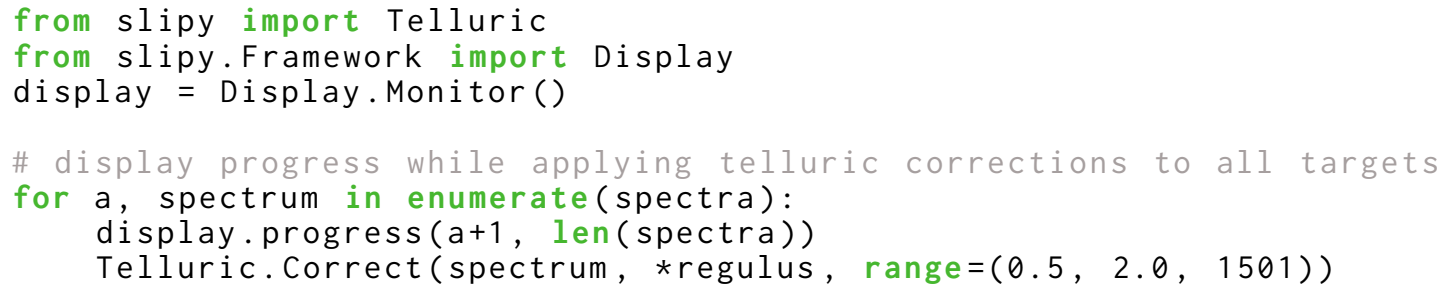

In the above code snippet we are applying the telluric correction procedure to all the spectra using the Regulus spectra from the previous examples. In this case, the density of the amplitude trials has been increased by a factor of ten, resulting in about a one second computation time for each spectrum. Figures 3.1 and 3.2 showcase the results of the Telluric.Correct() algorithm for two stars. HD 192640, an A2V variable star at $20^{h}$ $14^{m} 32.0^{s}+36^{\circ} 48^{\prime} 22.7^{\prime \prime}$ approximately $11^{\circ}$ southeast of $\delta$ Cygni, is shown to have a broad stellar component and a strong interstellar component for $\mathrm{Na}$ I D1 and D2 in Figure 3.1. In Figure 3.2 is Altair (HD 187642), another A type star, which has no interstellar features. Suffice it to say that Altair itself could potentially be a candidate for a reference spectrum for telluric corrections. When the algorithm is given this spectrum it effectively removes all features - behavior we would expect of a successful implementation of a telluric correcting algorithm. ${ }^{3}$

\footnotetext{
${ }^{3}$ Even better, if you give any one of the Regulus spectra as the first argument to the function you will get
} 


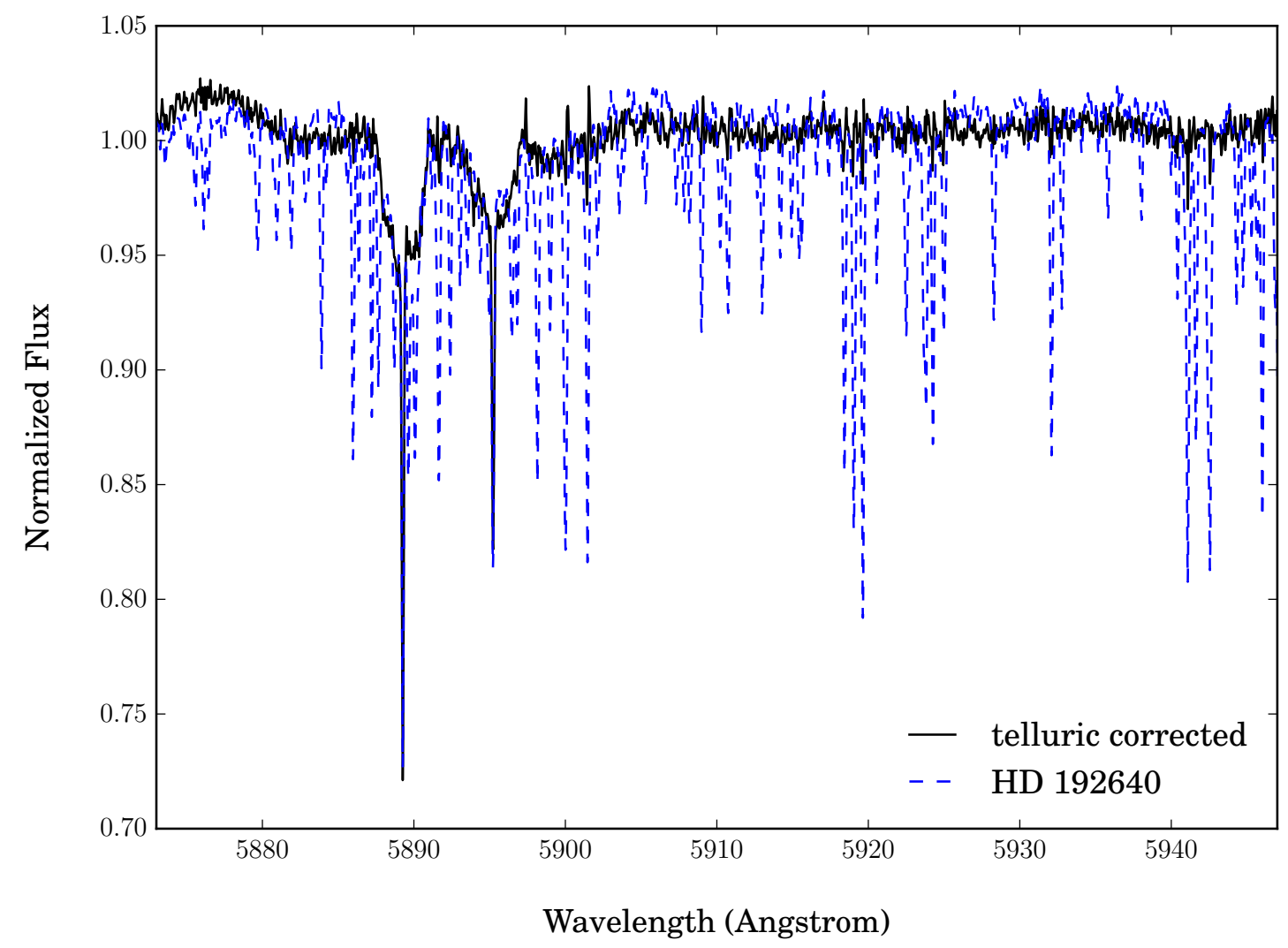

Figure 3.1: The above figure showcases the Telluric.Correct() routine. The spectrum is of HD 192460 (b03 Cyg), an A2V variable star. As in the entire analysis for this study, Regulus ( $\alpha$ Leo), a B8IV star, was used as the calibration spectrum. The Elodie archive has six spectra; the best fit is automatically chosen by the algorithm. 


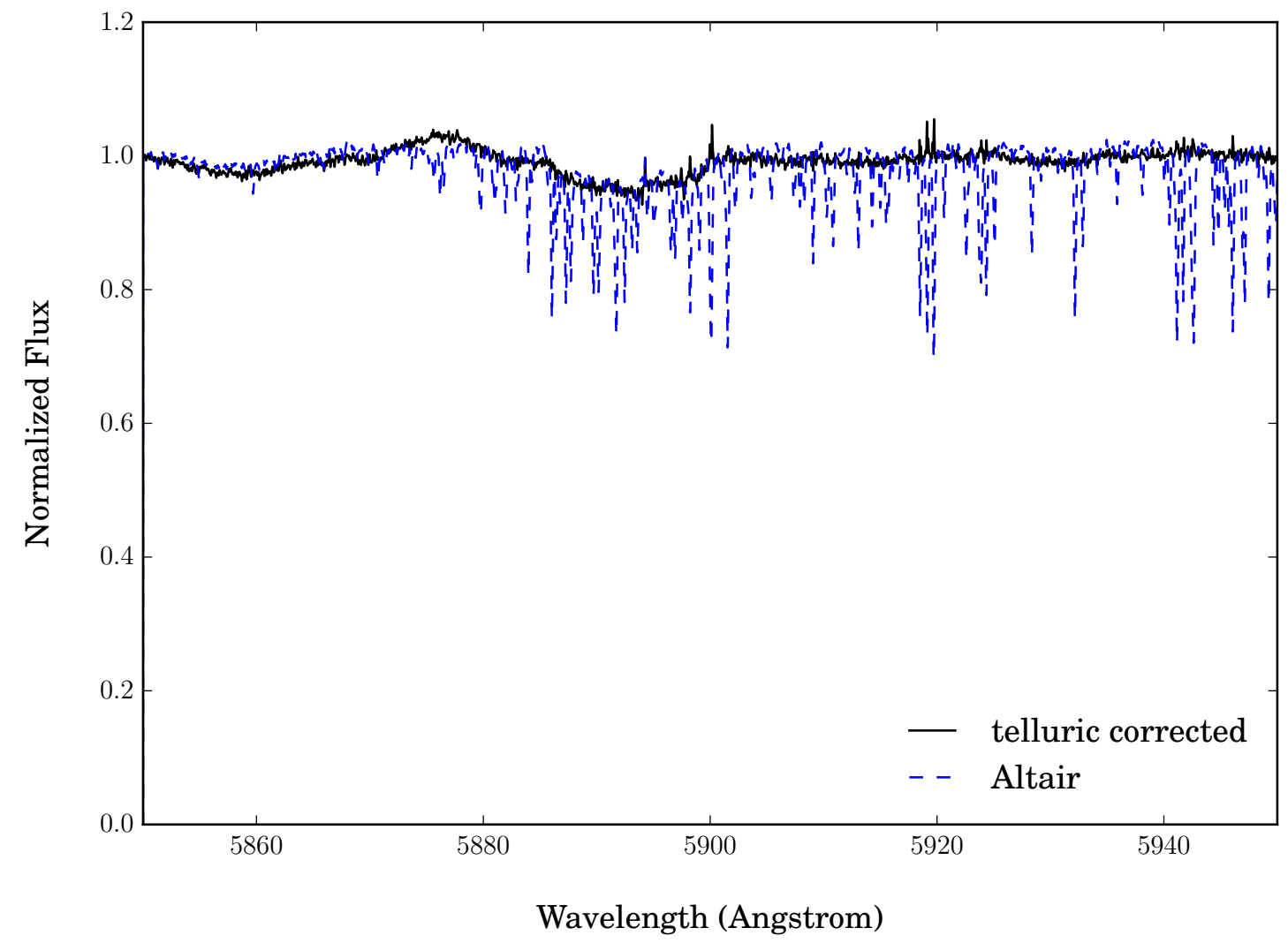

Figure 3.2: The above figure is an empirical test of the Telluric.Correct() routine. Here, a spectrum of Altair (HD 187642) was corrected against the same six spectra of Regulus as in Figure 3.1. As an A7V star, Altair has no absorption features in the above wavelength domain (with the exception of absorption due to Earth's atmosphere (telluric) and weak broad stellar absorption). By applying the routine to a star that might otherwise be a suitable candidate for a calibration spectrum itself, we have effectively removed every feature. 


\subsection{Heliocentric Velocity Corrections}

\subsubsection{Description}

Another significant calibration to be performed is a velocity correction. The Earth is in continual motion, with both rotation and revolution. In order to un-bias the measurement of the velocity of an absorption line in spectra, we must subtract out the motion of the observer along the line of site to the target. The largest influence is from the motion of the Earth around the Sun and can cause a shift up to approximately $30 \mathrm{~km} \mathrm{~s}^{-1}$. A successful algorithm for computing the velocity correction along the line of site to a target has been implemented since the 80s (Stumpff, 1980). The P. Stumpff algorithm is used in the rvcorrect task for IRAF. There already existed an implementation of this algorithm for Python ${ }^{4}$ maintained by Dr. Sergey Koposov. ${ }^{5}$ The accuracy of this is expected to be no worse than $\sim 1 \mathrm{~m} \mathrm{~s}^{-1} .{ }^{6}$

The algorithm computes the velocity vector of the observer due to the Earth orbiting the Sun, the motion from the Earth - Moon system, as well as the rotational velocity of the Earth. This vector is usually either with respect to the Sun, heliocentric, or with respect to the gravitational barycenter of the solar system, barycentric. The correction is the projection of this vector along the line of sight to the target. The necessary information to computer this correction is the latitude, longitude, and altitude of the observatory; the right ascension and declination of the target; and the Julian Day ${ }^{7}$ at the middle of the exposure. For this study, the heliocentric velocity is used.

\footnotetext{
back a spectrum identically one at all wavelengths!

${ }^{4}$ Specifically for Python 2, it was necessary to modify many of the files provided in the library to conform to Python 3.

${ }^{5}$ Institute of Astronomy, University of Cambridge

${ }^{6}$ At the very least, the performance of the system put together within SLiPy was tested against the output of the rvcorrect task within IRAF and found to be consistent for the same import parameters.

${ }^{7}$ The Julian Day is a non-relative measure of the exact time an event occurs, and is defined as the count of days from 12 noon on 1 January, $4713 \mathrm{BC}$. The fraction of the current day is simply expressed as the decimals following the count.
} 


\subsubsection{Implementation}

The Velocity.HelioCorrect() and Velocity.BaryCorrect() functions provide this capability. These functions provide a high level interface to the helcorr() function maintained by Dr. Koposov. helcorr() relies on a number of other modules all housed inside a library, astrolibpy. A modified version of this entire library is distributed with SLiPy (as a sub-package). The Velocity.HelioCorrect() and Velocity.BaryCorrect() functions access helcorr() in a more abstracted way. All the necessary arguments are condensed into two, an observatory and a spectrum. The observatory is an object that has the latitude, longitude, and altitude information. The spectrum argument should be of type Spectrum and have the right ascension, declination, and Julian Day of observation attached. ${ }^{8}$

In a similar design strategy as in IRAF, an Observatory is a thing that has attributes such as .latitude, .longitude, .altitude, etc. By default, there are 70 observatories defined. The 69 observatories (and attributes thereof) defined within IRAF's observatory database (usually under noao\$lib/obsdb.dat) have been adapted to an object oriented pattern within SLiPy. The additional observatory is for the present study. It and any additional observatories can be defined as follows:

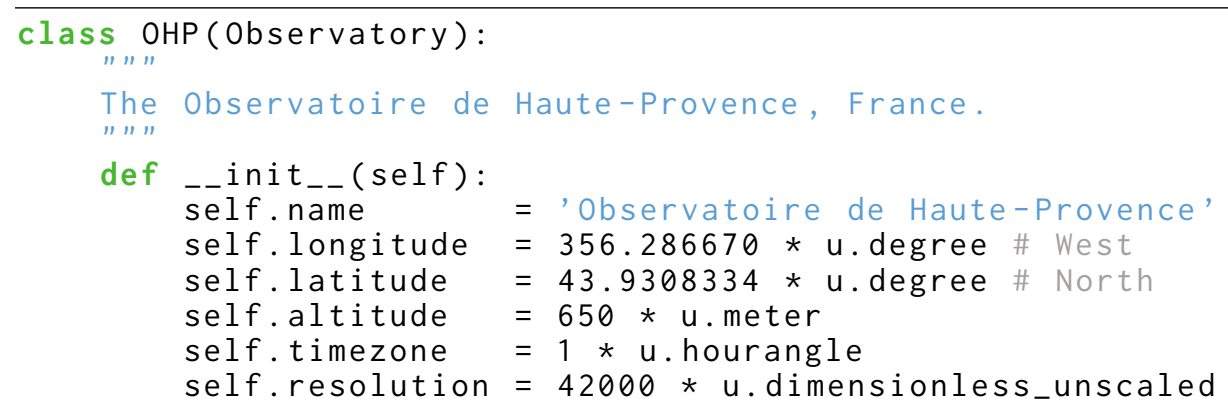

In the above snippet "Observatory" is an abstract base class that can/should not be

\footnotetext{
${ }^{8}$ In Python, everything is a first class object and is dynamic. I can attach a new data member to anything by simply assigning it (e.g., spectrum. $r a=20$ ).
} 
instantiated alone. The velocity correction functions check to see if the observatory the user provided is derived from this. Units are demanded for all the arguments' attributes, such that they can be properly converted if necessary to that needed for the P. Stumpff algorithm. Below is an example usage of the Velocity.HelioCorrect() function. As in the Telluric.Correct() function, the Spectrum argument(s) provided are altered; nothing is returned. As mentioned previously, the metadata for each observation needs to be attached to the spectra.

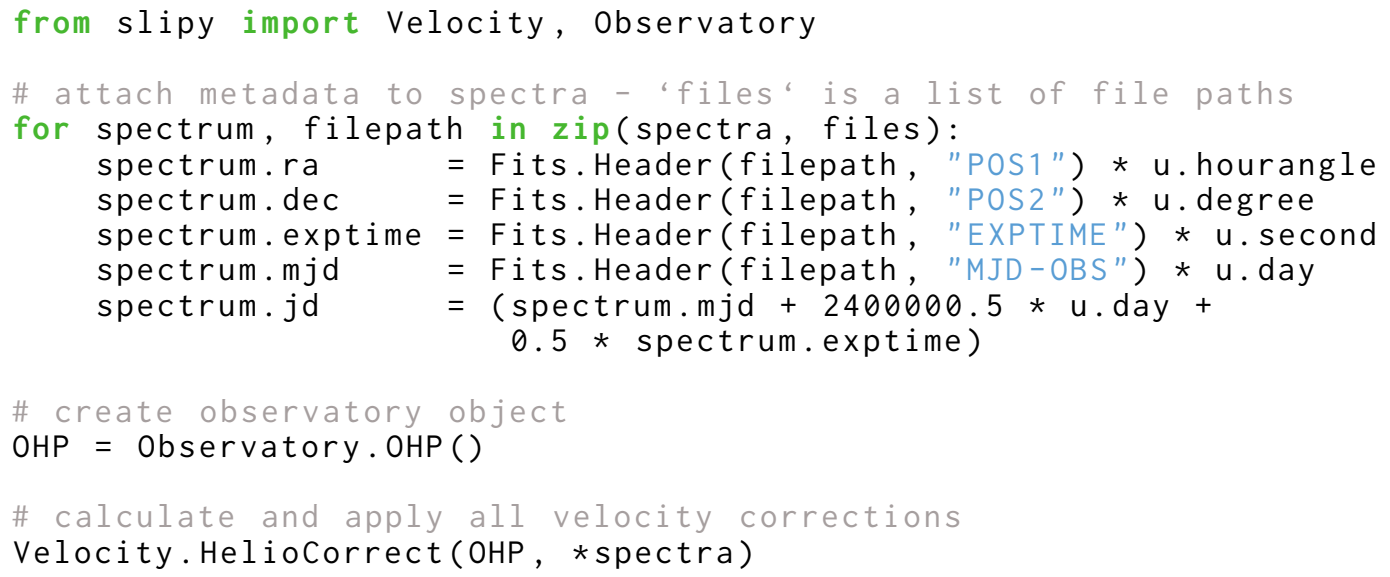

In the above snippet, the Modified Julian Day is taken from the header files because it is provided for the ELODIE files. The Julian Day at the middle of the exposure is computed using this. All data used in this study was calibrated in a similar manner. 


\section{CHAPTER 4}

\section{MEASUREMENTS}

The primary purpose of this study was to identify either the definitive presents or absence of the Sodium D lines; and in their presents, measure such quantities as equivalent width, column density, velocity, and the broadening parameter. This chapter covers the several distinct components involved in doing so, including the modeling of the continuum over the line, fitting and/or deblending multiple line components, and calculating these quantities from the extracted line profile.

\subsection{Non-parametric Gaussian Kernel Regression}

In spectra such as these, the absorption line features come down from a background continuum level and we must model this curve over the span of the line in order to integrate over that line. The continuum will have noise however and is almost never smooth. Generally, we solve for a smooth curve through the noise and interpolate over the line gap. There are of course numerous different methods and techniques for doing both of these. Here, we have chosen to use kernel regression to both simultaneously smooth out the continuum and gain information about the error in our measurements from the noise in the data. Gaussian kernel smoothing is a popular approach and is generally applicable with a balance between performance and complexity.

SLiPy contains a sub-package, Algorithms, which currently houses two modules:

Functions and KernelFit. It was convenient to abstract away the interface to a number of 
profile functions. The Gaussian, Lorentzian, and Voigt functions are all defined (along with variants and normalized versions thereof) within the Functions module. Within the KernelFit module is the KernelFit1D object. Most of the functionality discussed in the rest of this chapter is defined in the Profile module, whose functions use the kernel fitting algorithm.

Here, we use the Nadaraya-Watson kernel regression method. Given a discrete data set of $(x, y)$ elements with sufficient noise, we can estimate its real valued function as

$$
f(x)=\frac{\sum_{i} k\left(x-x_{i}\right) y_{i}}{\sum_{i} k\left(x-x_{i}\right)} \quad \text { where } k(x)=A \exp \left(-\frac{x^{2}}{2 \sigma^{2}}\right)
$$

This is essentially an arithmetic mean of the data set but with weights determined by the proximity of that data point from the point in question. The Gaussian kernel, $k$, has a length scale (or bandwidth), $\sigma$. This is an important thing to understand because it must be specified by the user. When trying to model the continuum and extract the line profile from the data, the length scale must be sufficiently large as to not trace the noise yet not so large as to fail to follow the bulk shape of the curve. This is generally true of any application using kernel smoothing. For most of the ELODIE data used in this study a bandwidth of approximately $0.08 \AA$ was optimal. An example usage of KernelFit1D is available in Figure 4.1

\subsection{Spectral Plots}

Before continuing on with this discussion, the SPlot (\€s plät) object needs mentioning. The Profile.Select(), Profile.Extract(), Profile.AutoFit(), and

Profile.MultiFit() functions each offer a graphical interface for the user to select data as a sample of the continuum and the line contained within. The arguments to these functions 


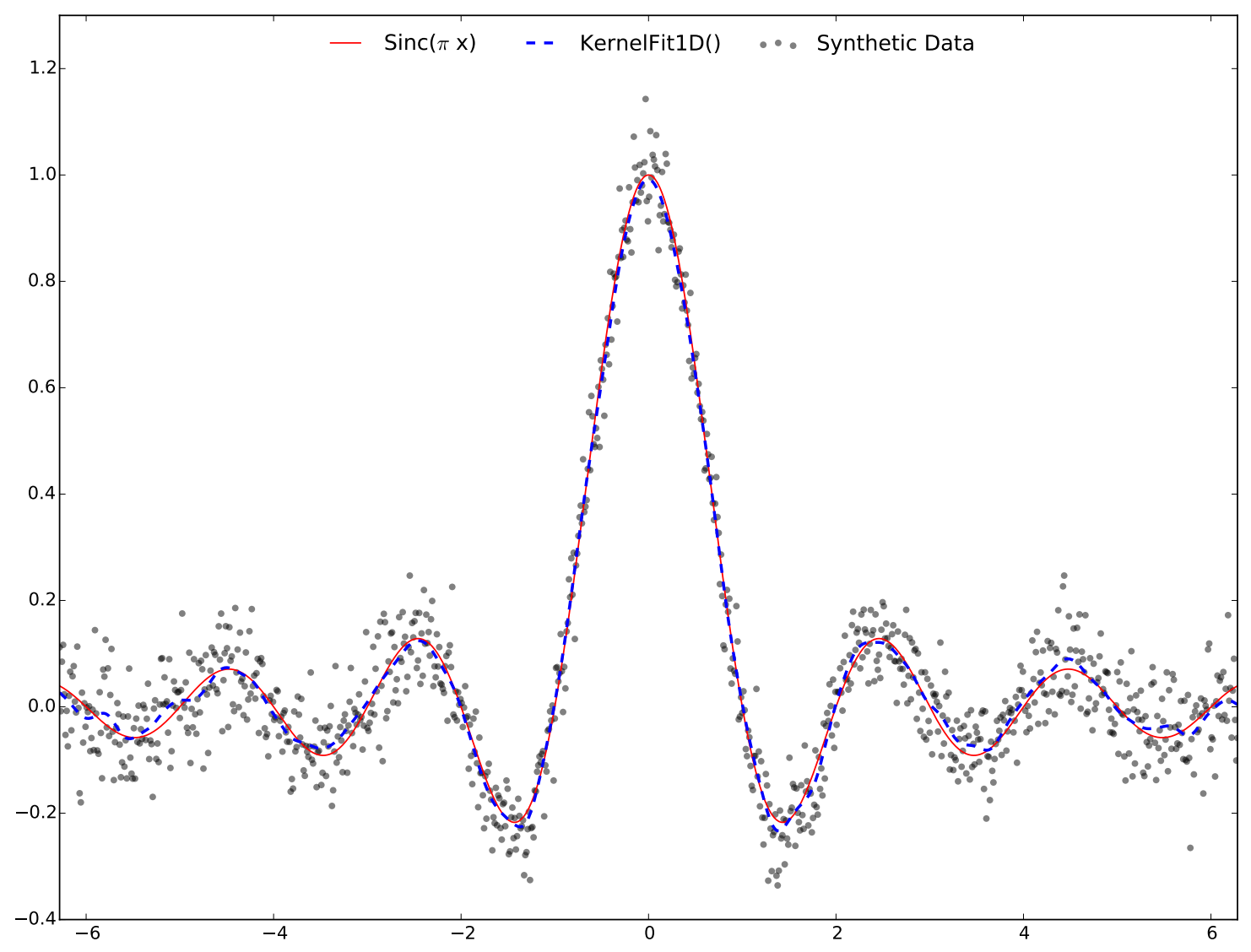

Figure 4.1: The above figure showcases KernelFit1D in a best-usage scenario. Here, we have a synthetic data set constructed of a normalized sinc function with added both normal white and red noise. The analytical curve is shown with a thin solid red line. The bold blue dashed line is the solution using the kernel smoothing algorithm with a bandwidth of $\pi / 32$. 
are all of type SPlot. ${ }^{1}$

An SPlot, or Spectral-Plot, is a manager class for creating and maintaining figures using matplotlib (Hunter, 2007). Generally, using the matplotlib.pyplot module allows one to plot points and curves of $x-y$ data. The $x$ and $y$ limits can be set, axis labels and legends (or other text) can be set. What an SPlot does is to retain all the calls to the most common methods applied using the pyplot module and allow you to pass around figures as objects. The full documentation for this feature will not be provided here (it can be accessed online), but an example should suffice. The results of the below snippet are in Figure 4.2.

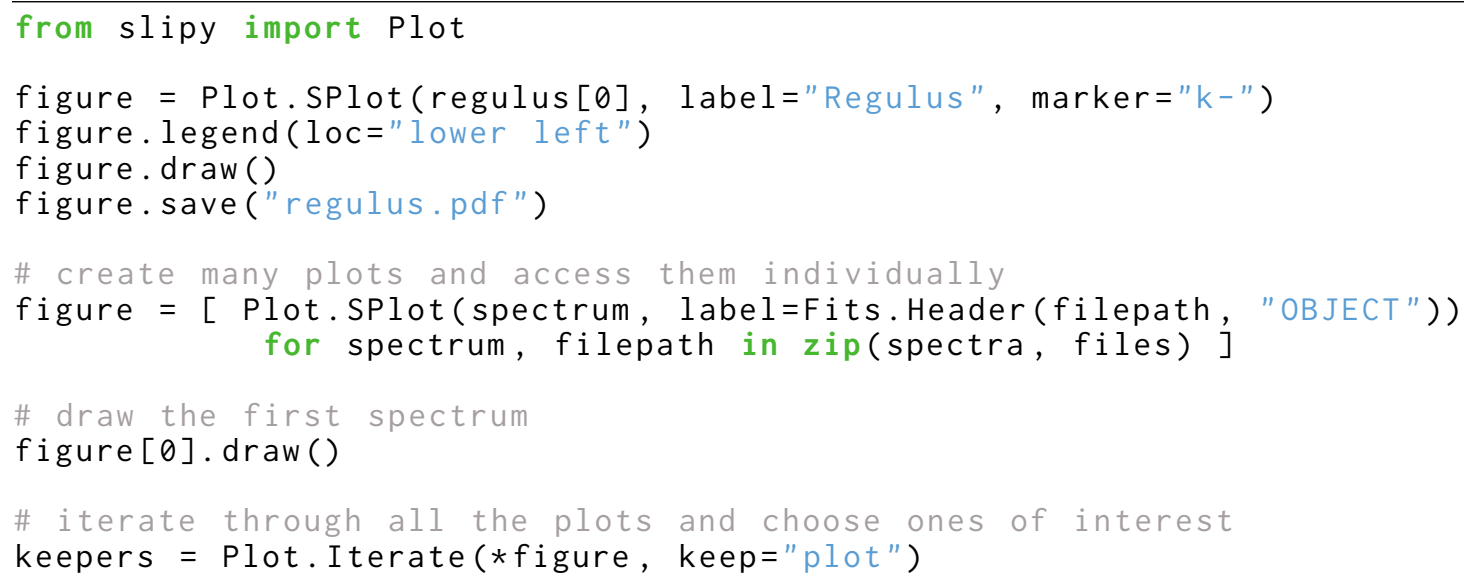

\subsection{An Interactive Graphical User Interface}

The principle function of the Profile module is the MultiFit() routine which creates, maintains, then destroys a FittingGUI and then takes the measurements for all the lines extracted. The following subsections outline some of the major areas involved with this. The FittingGUI uses the API provided by matplotlib to add widgets to the SPlot figures. The goal here is to be able to interactively fit profiles to data with one or more components.

\footnotetext{
${ }^{1}$ Although, they may alternatively be of type Spectrum for which a generic SPlot will be created.
} 


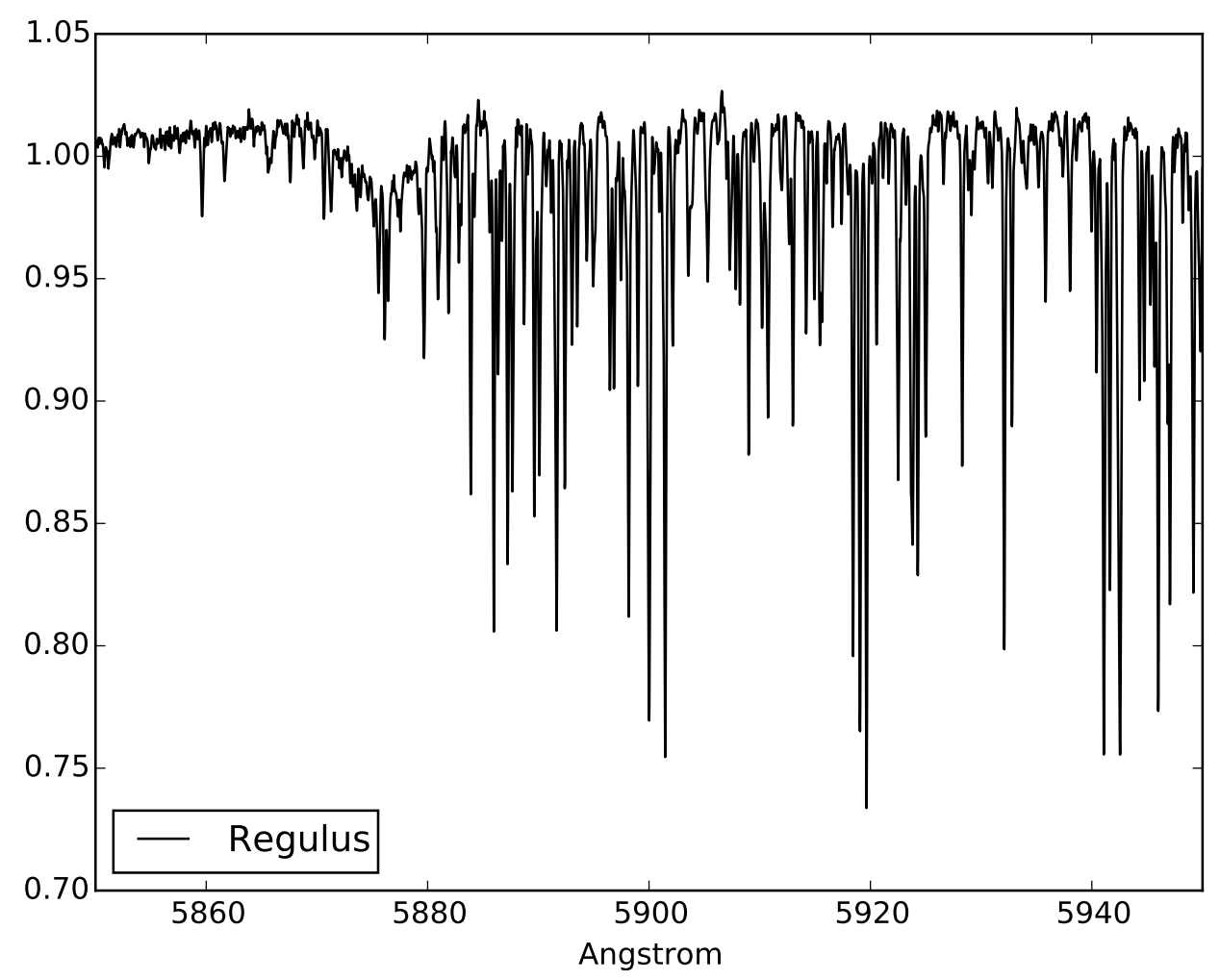

Figure 4.2: The output file from the example usage of the SPlot object. 


\subsubsection{The Voigt Profile}

Generally, the FittingGUI has been programmed to have the capacity to model Gaussian, Lorentzian, and Voigt line shapes. For absorption lines (barring some more complex scenarios such as rapid rotators, etc.) we expect to see Voigt profiles. This is the result of an intrinsic line profile well approximated by a Lorentzian ${ }^{2}$ that is broadened with the convolution of one or more Gaussian profiles. The first Gaussian is physical and due to both thermal (Doppler broadening) and turbulence. The second Gaussian is due to the blurring from instrumental effects. ${ }^{3}$ Together, the Voigt profile can be expressed as (Draine, 2011)

$$
\phi_{\nu}^{\text {Voigt }} \equiv \frac{1}{\sqrt{2 \pi}} \int \frac{d v}{\sigma_{v}} e^{-v^{2} / 2 \sigma_{v}^{2}} \frac{4 \gamma_{u l}}{16 \pi^{2}\left[\nu-(1-v / c) \nu_{u l}\right]^{2}+\gamma_{u l}^{2}}
$$

where the integral is over velocity and $\nu_{u l} \equiv\left(E_{u}-E_{l}\right) / h$ crosses the transition between energy levels $u$ and $l$. The width of the Lorentzian reflects the fundamental uncertainty in the finite lifetimes of the energy levels against transitions to all other levels. The intrinsic width of the absorption line is related to $\gamma_{u l}$ and the Einstein coefficient $A_{u l}$ as

$$
(\Delta v)_{\mathrm{FWHM}}^{\mathrm{intr.}}=\frac{\gamma_{u l}}{2 \pi}=\frac{\lambda A_{u l}}{2 \pi}
$$

The strength of the absorption transition can also be described by the oscillator strength,

$$
f_{u l} \equiv \frac{m_{e} c}{\pi e^{2}} \int \sigma_{l u}(\nu) d \nu
$$

where the monochromatic absorption cross section $\sigma_{l u}(\nu)$ to a normalized line profile $\phi_{\nu}$ is

\footnotetext{
${ }^{2}$ The line profile is better approximated by the Kramers-Heisenberg formula (Draine, 2011).

${ }^{3}$ Though it is not necessarily the case that an instrumental line-spread function (LSF) actually be a Gaussian, this is often sufficient.
} 


$$
\sigma_{l u}(\nu)=\frac{g_{u}}{g_{l}} \frac{c^{2}}{8 \pi \nu_{l u}^{2}} A_{u l} \phi_{\nu} .
$$

These quantities, $A_{u l}$ and $f_{u l}$, are import to relating our Voigt line profile back to physical properties.

These are analytical expressions; the Profile module within SLiPy relies on the relationship between the Voigt function and the Faddeeva function (a complex complementary error function). The Voigt profile can be expressed as the real part of this function, $w(z)$, as

$$
V(x ; \sigma, \gamma)=\frac{\operatorname{Re}[w(z)]}{\pi(\sigma \sqrt{2 \pi})} \text { for } z=\frac{x+i \gamma}{\sigma \sqrt{2}}
$$

Within the SciPy library there is a scipy.special module with a numerical implementation provided for $\operatorname{wofz}() .{ }^{4}$ The profiles fit in this study come from this approach. In reality, it is only the absorbers whose profile is Voigt in shape. The actual feature in spectra is an exponential relationship, $I_{\nu}=I_{0, \nu} \exp \left(-\tau_{\nu}\right)$. For only small absorption features, this is approximately linear. Using something called the curve of growth (Draine, 2011) one can detangle the relationship between the equivalent width and the number of absorbers in a medium. Alternatively, we will employ the so called apparent optical depth method (Savage and Sembach, 1991) for non-optically thin lines; more on this in the following sections.

\subsubsection{Atomic Ions}

The Na I (D1 and D2) lines have oscillator strengths 0.320 and 0.641, respectively (Morton, 2003) and transition probabilities (Einstein coefficients) $6.14 \times 10^{7} \mathrm{~s}^{-1}$ and

\footnotetext{
${ }^{4}$ This is essentially $\exp (-z \star * 2) \star \operatorname{erfc}(-i * z)$. Please see the Steven G. Johnson, Faddeeva W function implementation, http://ab-initio.mit.edu/Faddeeva, for more details.
} 
$6.16 \times 10^{7} \mathrm{~s}^{-1}$, respectively (Kramida et al., 2014). This relates to the FittingGUI in that the user provides the information on both of these quantities as a single ion argument. So in fitting a particular absorption line in your data, you only need to specify what ion you are fitting and it knows the wavelength, fvalue, and $A$ (in both air and vacuum) for most ions with wavelengths long-ward of the Lyman limit.

Within the SLiPy.Data sub-package is an Atomic module that not only incorporates the entire data set from Morton (2003) but defines the Atomic.Ion object. For the sake of brevity, this functionality will not be documented entirely here, suffice it to say however that it's a very high level aspect of the library to not require the user to look up these values as the most recent published data is already built in to the software. ${ }^{5} \mathrm{~A}$ short example is presented here, and again in the later example using the Profile.MultiFit() function.

from slipy. Data import Atomic

\# find oscillator strengths for C III ions

Atomic.IonSearch ("C III")

[(<Quantity 977.0201 Angstrom>, 0.7570010842747638$)$,

(<Quantity 1908.734 Angstrom>, 1.6875419997146983e-07)]

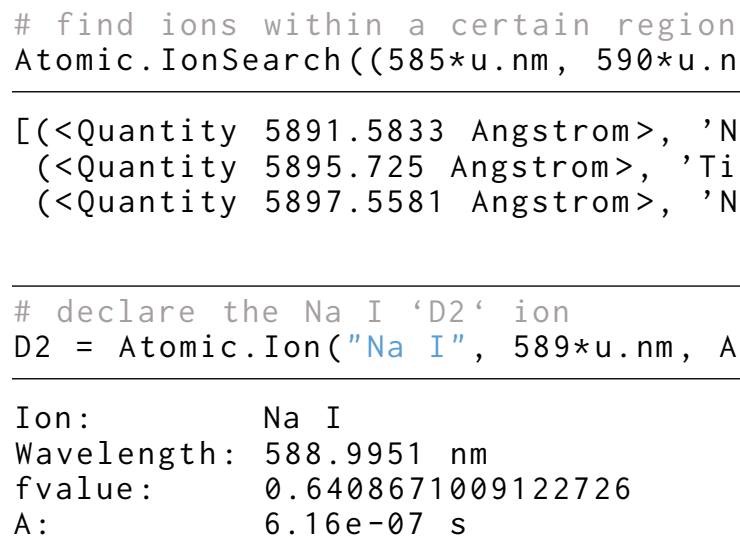

\footnotetext{
${ }^{5}$ While the oscillator strengths for these ions is part of the software, as of today the transition probabilities
} have not been built in and must be specified by the user. 


\subsubsection{Fitting Absorption Line Parameters}

With an ion declared we can pass it along with an splot, an observatory, ${ }^{6}$ and a bandwidth to Profile.MultiFit(). The first thing that happens is you are asked to select four data points along the spectrum. This is to mark the edges of the line in addition to samples of the continuum on either side. KernelFit1D models the continuum and the Profile.Extract() routine is run. With a separated line and continuum (interpolated ${ }^{7}$ over the absorption line gap) you are then asked to select more data points along the line to mark the suspected peaks of one or more components.

The plot moves up in the figure and sliders appear for a number of parameters along with radio buttons for each line selected. Above the sliders and below the plot is a preview of the absorption line parameters for each Voigt profile that is now plotted for each component. These steps are illustrated in Figures 4.3 - 4.6. What sliders appear depends on the function requested ("voigt" by default) and whether or not you provide an observatory and an ion. In this context, by providing both of these, gamma does not appear as an alterable parameter. This is because the transition probability provided by the ion solely determines the expected FWHM of the intrinsic profile and the only parameter to be fit is an observed sigma.

The broadening parameter can be extracted from the observed sigma as the convolution of one Gaussian on another is simply another Gaussian with

$$
\sigma_{\text {convolution }}^{2}=\sigma_{a}^{2}+\sigma_{b}^{2}
$$

So the width of the thermal/turbulance Gaussian is

\footnotetext{
${ }^{6}$ This should be either one of the defined Observatory objects or something derived thereof. We pass this to Profile.MultiFit() with an attached .R (resolution) so we know the FWHM of the instrumental Gaussian profile.

${ }^{7}$ The default is to use natural cubic spline interpolation, though alternatives can be requested by passing the kind keyword argument (see scipy.interpolate.interp1d for details).
} 


$$
\sigma_{\text {therm }}=\sqrt{\sigma_{\text {observed }}^{2}-\sigma_{\text {instrument }}^{2}}
$$

where the resolution of the instrument gives the width,

$$
\begin{aligned}
(\Delta \lambda)_{\mathrm{FWHM}} & =\frac{\lambda}{R} \\
& =2 \sqrt{2 \ln 2} \sigma_{\text {instrument }}
\end{aligned}
$$

and the broadening parameter is,

$$
b \equiv \sqrt{2} \sigma_{\text {therm }} .
$$

While the calculations are performed perpetually during the fitting process, a final calculation of the equivalent width and column density are performed after the fitting process is complete, attached to each returned line as $\mathbf{. W}$ and $\mathbf{. N}$ respectively of Measurement types. ${ }^{8}$ Also, the .parameters for the Voigt profile and the velocity, .v, and the broadening parameter, $\mathbf{. b}$, are attached to each line.

The details behind the calculation of the equivalent width and column density is reserved for the following subsections. An example usage of the Profile.MultiFit() function is presented here. and Figures 4.3 - 4.6 showcase individual steps.

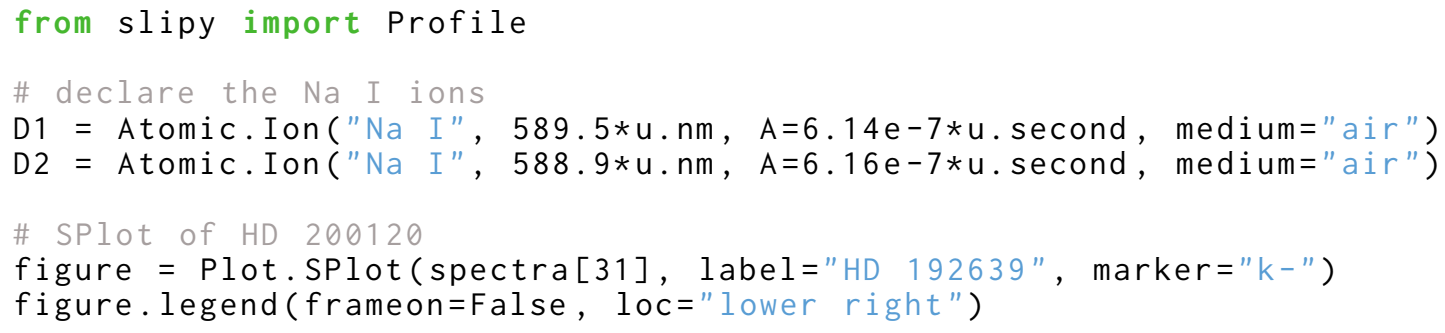

${ }^{8}$ The Measurement class is unique to SLiPy and is derived from a Quantity but has a .name, .uncertainty, and .notes. It can be operated on the same as a Quantity (in fact it decays to one afterwards). This functionality is in the works by contributors at Astropy by is not currently available there. 


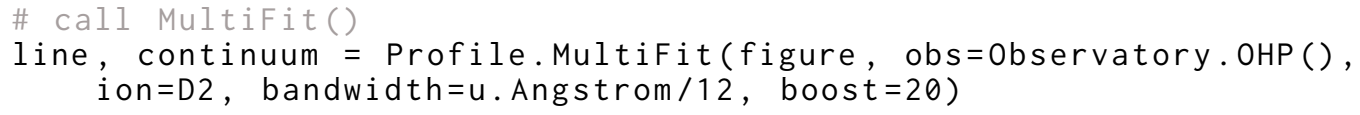

In the above snippet, the spectrum could have been passed directly to the function and the SPlot would have been created automatically. The idea here is that any SPlot can be customized and passed to the function and it will retain its attributes (e.g., we could have set the marker to "k+" and crosses would have been used for the data points and not an interpolated curve or we could have used overlay() to add more than one Spectrum to the same figure and fit them simultaneously!). The extra keyword argument, boost, is an option that artificially inflates the number of pixels in order to increase the accuracy of the numerical integration. ${ }^{9}$ This is not performed during the fitting process (the preview does not boost the resolution) which is why the attached calculations might be slightly different than the previewed results.

\subsubsection{Equivalent Widths}

The equivalent width, $\mathrm{W}$, of an absorption line is a good measure of the strength of a line, or power removed by the absorbers (Draine, 2011), and is measurable even in the face of lower resolution data or high thermal broadening. It can be expressed in a few different ways but generally is the width a line would have if it maintained the same integrated area but a height equal to that of the continuum level. It is most accurately calculated by numerically integrating the Voigt profile.

$$
\begin{aligned}
W & =\int\left[1-\frac{I(\lambda)}{I_{0}(\lambda)}\right] d \lambda \\
& =\int\left[1-e^{-\tau(\lambda)}\right] d \lambda
\end{aligned}
$$

\footnotetext{
${ }^{9}$ The numerical integration uses the composite Simpson's method (see scipy.integrate.simps). This is very accurate even at moderate resolution, but more elements gives more accurate results.
} 


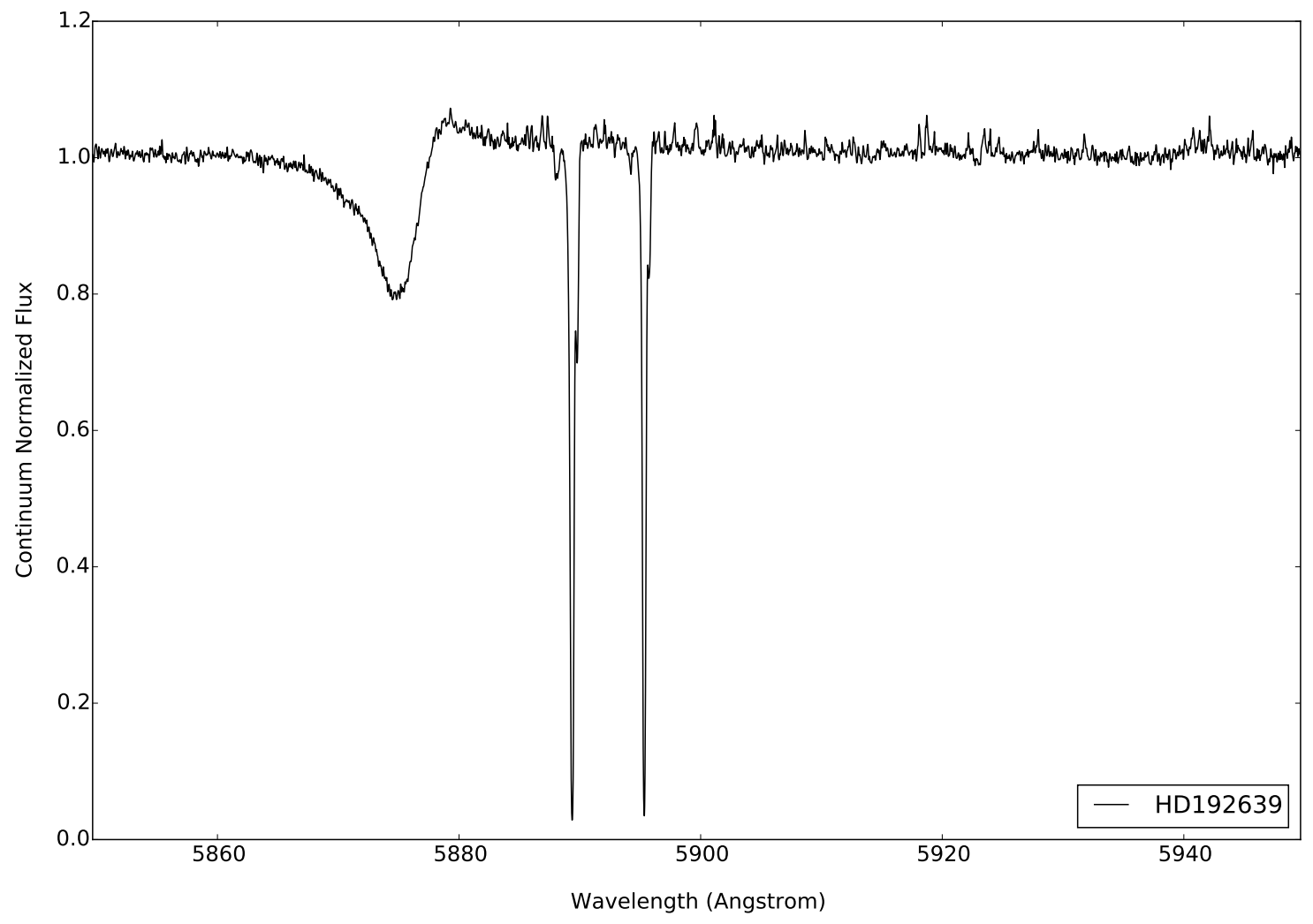

Figure 4.3: The blue supergiant HD 192639 is represented by an SPlot using SLiPy. Here we can see more than one interstellar Na I component. The SPlot itself can be passed as an argument to Profile.MultiFit(). 


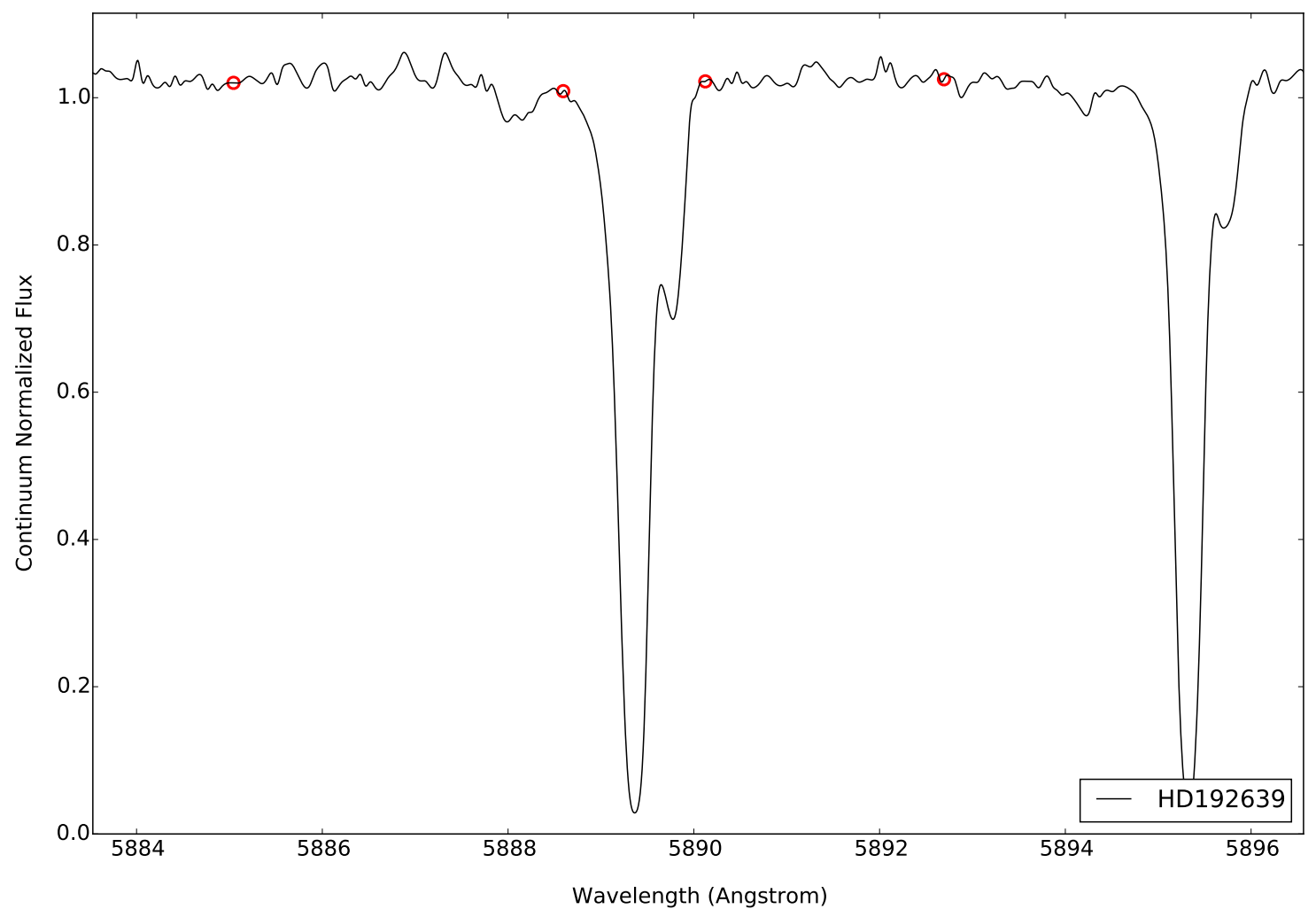

Figure 4.4: After passing the SPlot object from Figure 4.3 to Profile.MultiFit(), along with several necessary additional arguments, we are prompted to select four points along the spectrum to mark the boundaries of the line(s) and a sample of continuum on either side. The KernelFitID routine is used to smooth out the continuum and evaluate an RMS noise error. 


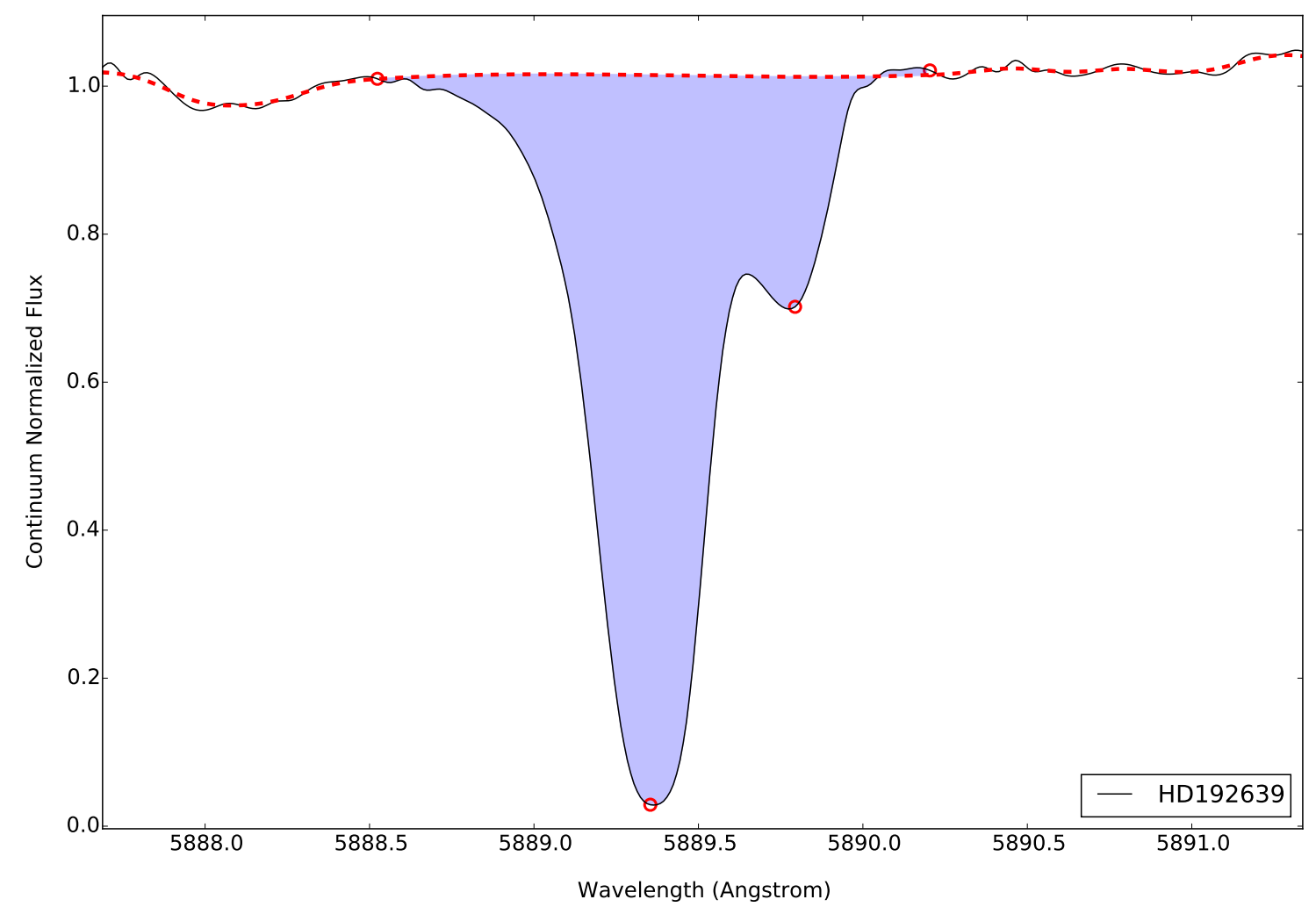

Figure 4.5: After extracting the line(s) from the continuum in Figure 4.4 we select the peaks of the absorption line(s). Buttons not shown in the figure allow the user to pan and zoom on the spectrum. It is unavoidable that the continuum interpolated over the line will be below the wings. This is because the noise in the continuum is assumed to be equally distributed above and below the continuum. By smoothing out the continuum we draw a curve through the noise and the interpolated curve between the samples will be continuous, in the mathematical sense. The idea is that we can get the non-linear aspect of the continuum over the line even if it is too low at first. In the next step we can raise the overall continuum level using a slider. 


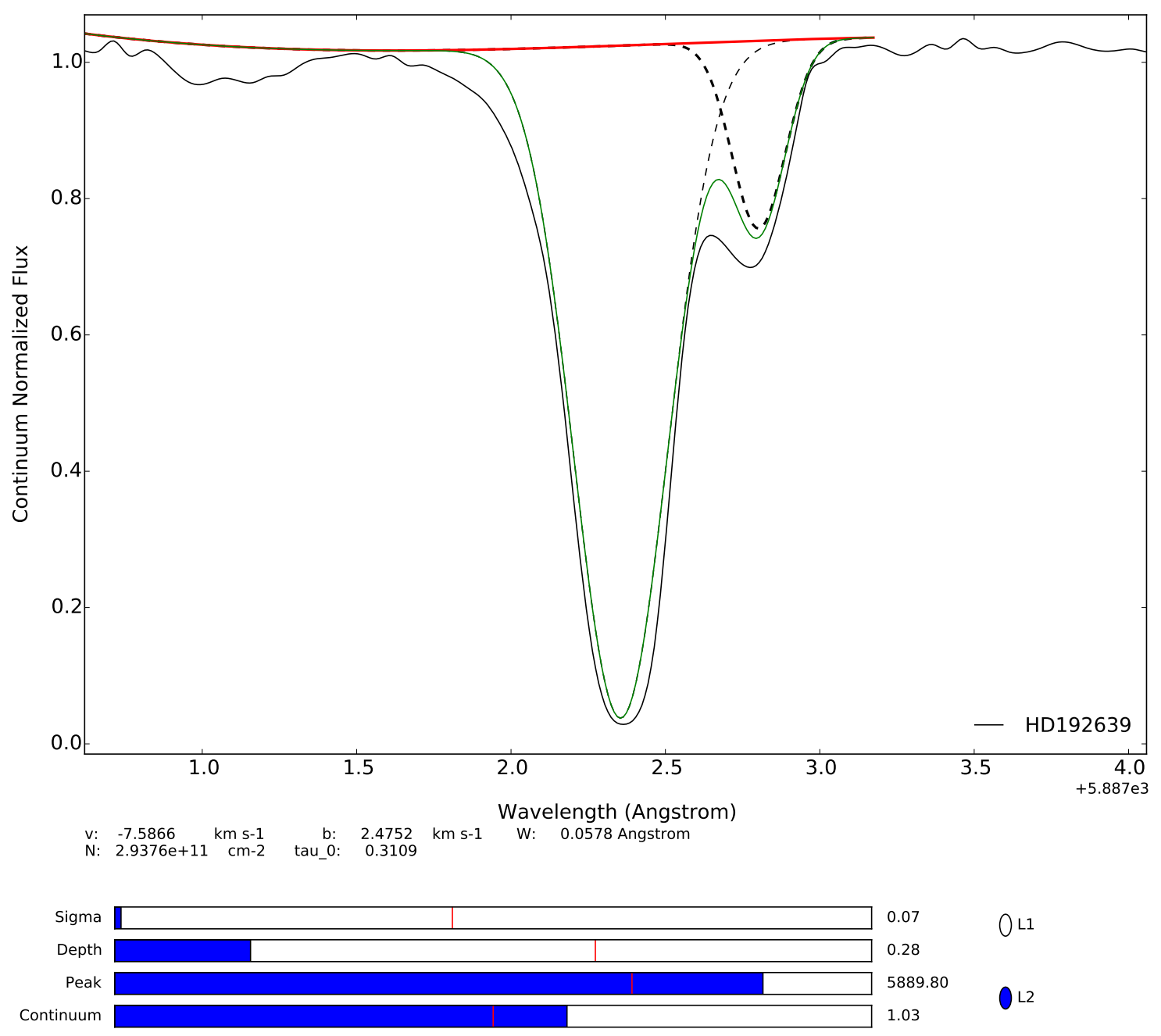

Figure 4.6: The last phase of the Profile.MultiFit() routine creates an analytical profile (as a Voigt function by default) for each selection made. Above is a mid-session view during the fitting process. The currently selected line is represented by a bold dashed line. To select a different line, choose the corresponding radio button in the bottom right. The continuum fit in the previous step is represented by a solid red line and the blended (superposition) line is solid green. The sliders created (bottom left) depend on the function argument. When an ion and observatory are given with appropriate member attributes, a Voigt profile is used and it is assumed that we are in fact trying to fit absorption lines in astronomical spectra (generally, this routine can be used outside of this context). In this context the velocity, $v$, the broadening parameter, $b$, and the apparent column density, $N$ are previewed between the sliders and the active plot. The sliders allow for a range of possible values that are reasonable for the lines marked. 
where $I_{0}$ is the continuum and $\tau(\lambda)$ is the optical depth of the line,

$$
\tau(\lambda) \equiv \ln \left[I_{0}(\lambda) / I(\lambda)\right]
$$

Within SLiPy, both the Profile.EquivalentWidth() and Profile.ColumnDensity() functions are extensions of the Profile.OpticalDepth() function. For a single, well behaved absorption line the Profile.Extract() function can be used alone without any fitting procedure and a line and continuum can be separated by selecting these regions from the splot given.

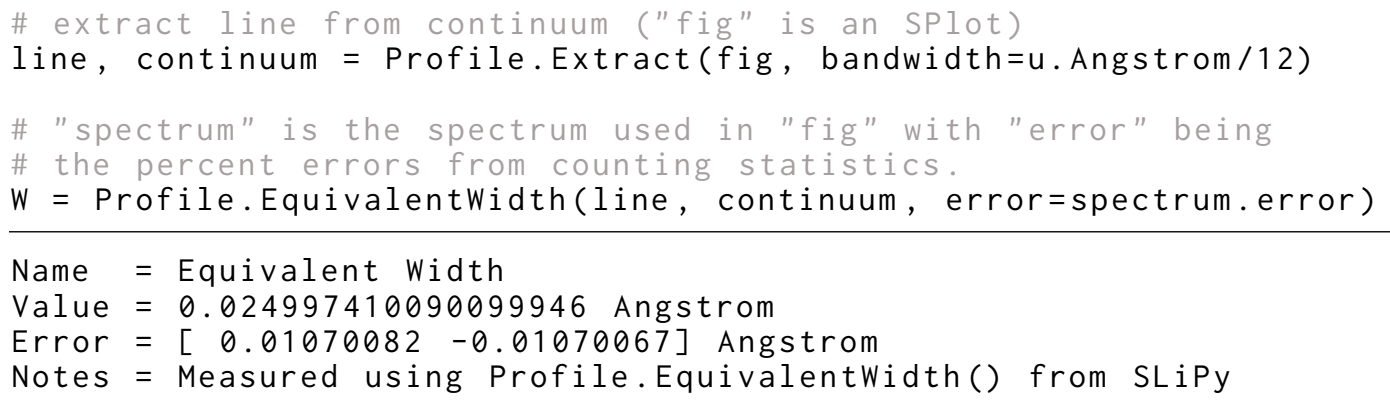

The above snippet is of HD 186155 and was measured to have an equivalent width for the D2 line of Na I of $(25.0 \pm 10.7) \mathrm{m} \AA .{ }^{10}$ Doing the same set of commands but instead selecting D1 gives us $(17.7 \pm 9.6) \mathrm{m} \AA$ and the ratio $W_{2} / W_{1} \simeq 1.41$. This puts it on the flat portion of the curve of growth and the stronger line has become more optically thick. As such, using the equivalent width to infer the column density no longer is accurate.

\subsubsection{Column Densities and the Apparent Optical Depth Method}

The column density is essentially a measure of the total number of absorbers along a column and is usually expressed as the number of atoms $\mathrm{cm}^{-2}$. With complementary observations, it can be used to understand the length of a cloud. In this case, given the

\footnotetext{
${ }^{10}$ This value of course depends on what is provided via a selection by the user!
} 
distance to a star for which there is interstellar absorption from the LCCC, the cloud's leading edge is that much closer. ${ }^{11}$ In the limit of optically thin gas, $\tau_{0} \ll 1$ (where $\tau_{0}$ is the optical depth at line center), the column density can be related to the equivalent width as (Draine, 2011)

$$
\begin{aligned}
N & =\frac{m_{e} c^{2}}{\pi e^{2}} \frac{W}{f_{l u} \lambda_{l u}^{2}} \\
& =1.130 \times 10^{12} \mathrm{~cm}^{-1} \frac{W}{f_{l u} \lambda_{l u}^{2}}
\end{aligned}
$$

There are several techniques for computing the column density of a line that might offer better results dependent on the circumstances. Here, I will review the essence of the so called "apparent optical depth method" as described by Savage and Sembach (1991). More directly, the apparent column density relates to the apparent optical depth as

$$
N_{a}(\lambda)=\frac{m_{e} c^{2}}{\pi e^{2} f_{l u} \lambda_{l u}^{2}} \tau_{a}(\lambda)
$$

The usage of the word apparent here embodies the understanding that with an instrumentally smeared line we are only measuring the observed absorption. In contrast with the equivalent width, the measure of column density is indeed sensitive to instrumental broadening.

In either case, there is not much that can be done for severely saturated lines. This was not the case for the majority of the lines of interest for Non-OB stars investigated in this study; however there were indeed absorption lines for which saturation was near complete. Saturation is when the optical depth of a line begins to approach one at line center, note that this often occurs well before an observed line shows a significant depth due to instrumental broadening. When photons propagate through the medium, as more

\footnotetext{
${ }^{11}$ Not to insinuate that the LCCC necessarily has a hard edge.
} 
are removed and scattered by the absorbers we cannot remove more photons that were there to begin with. So within the ISM, if the size or density of a cloud is sufficient, the absorption can be near complete before we even get all the way through. This scenerio spells doom to inferring information about the extent of the cloud. As the stronger line (in the context of doublets again) becomes optically thick and as saturation sets in we lose the ability to make accurate measurements. In principle, for doublet lines who differ in the product $f \lambda$ by a factor of two, we expect that the stronger line will have double the equivalent width and the same column density.

So long as the saturation is only modest, we can gain significant empirical information about the extent of saturation by measuring the difference in the observed line strengths (Savage and Sembach, 1991). Integrating the column density per unit wavelength gives the combined apparent column density,

$$
N_{a}=\frac{m_{e} c^{2}}{\pi e^{2} f_{l u} \lambda_{l u}^{2}} \int \tau_{a}(\lambda) d \lambda .
$$

The true column density can be estimated as

$$
\log N_{a}^{c}=\log N_{a}^{n-1}+\Delta \log N_{a}^{n-1}
$$

where $\Delta \log N_{a}^{n-1}$ is a correction factor determined from the relationship between $\log N_{\text {true }}-\log N_{a}{ }^{n-1}$ and the difference $\log N_{a}{ }^{n-1}-\log N_{a}{ }^{n} \cdot{ }^{12}$ Given a value for this difference, we can estimate the correction based on published values (Savage and Sembach, 1991).

The Profile.MultiFit() function currently approximates the apparent column density during the fitting procedure using the optically thin approximation and the relationship between the column density and the equivalent width. Afterward, the more

\footnotetext{
12 The $n$ and $n-1$ refer to the strong and weak line respectively for the doublet.
} 
accurate direct integration is performed using the Profile.ColumnDensity() function. The arguments consist of a line and a continuum, the same as for the Profile.OpticalDepth() and Profile.EquivalentWidth() functions. Here however we want to also provide a third, ion, argument (as described previously). Further, if we have already computed the apparent column density for the weaker of two lines of the same species, we can provide that measurement with the keyword argument weakline and the returned measurement will have the .correction and .true values attached. This uses the published corrections from Savage and Sembach (1991) and interpolates to estimate the correction given the calculated $\log N_{a}^{n-1}-\log N_{a}^{n}$.

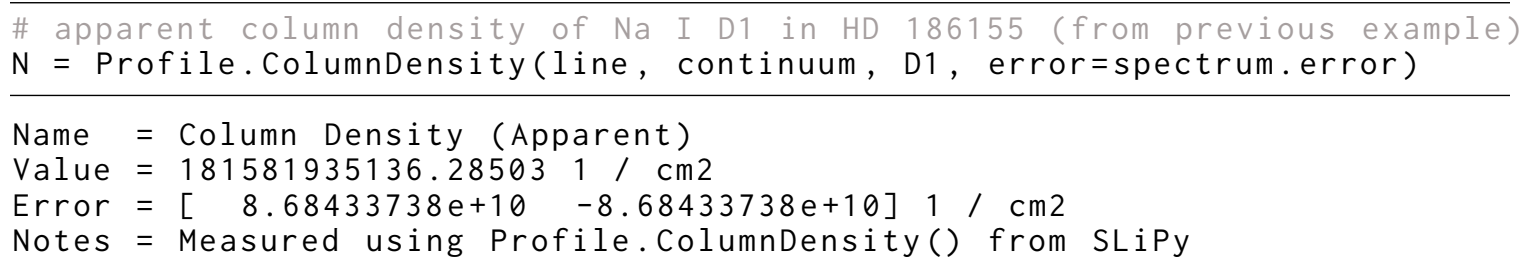

For only slightly saturated lines and/or not optically thin, we can now estimate the true column density by passing these results as the keyword argument, weakline, to

\section{Profile.ColumnDensity().}

\subsection{Uncertainty Calculations}

The uncertainties provided in this study were computed from both the noise in the continuum (RMS) and from counting statistics. Given a "raw" spectrum in counting units, the uncertainty in those counts goes as

$$
\delta s_{\text {count }}=\sqrt{s} .
$$

The uncertainty from these statistics is added in quadrature with those from the continuum fitting, 


$$
\delta s_{\text {total }}=\left[\delta s_{\text {count }}^{2}+\delta s_{\text {rms }}^{2}\right]^{1 / 2}
$$

Within SLiPy, either both or neither of these may be provided. The Profile.Extract() function (so in turn also Profile.MultiFit()), attaches an .rms value in like units directly to the continuum returned. The measurement functions look for this member variable and can handle either absolute or percent units. The uncertainties from counting statistics may be provided via the error keyword argument (as demonstrated by the last two examples). These work both in isolated usage and in connection with each other (adding in quadrature).

Preparing an error spectrum from an original spectrum of counts might be accomplished as in the following example.

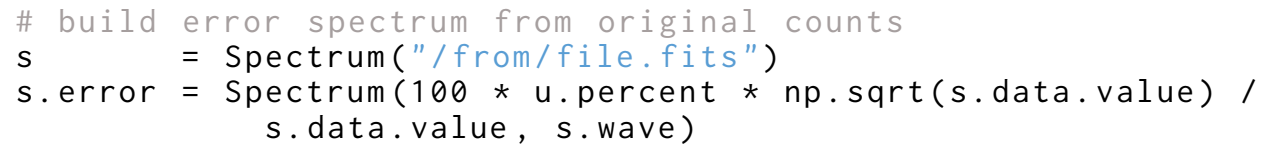

Be sure however to perform the same velocity corrections on both your spectra and corresponding error spectra. 


\section{CHAPTER 5}

\section{RESULTS}

In this chapter I provide both numerical data and visual representations of the results of this study. Figures 5.1 - 5.3 all map the stellar targets from ELODIE over a $40^{\circ}$ background sky centered on $\delta$ Cygni. ${ }^{1}$ Figure 5.1 shows all the stellar targets; Figures 5.2 and 5.3 show the definitive detection of interstellar sodium or lack thereof, respectively. The numerical annotations in these latter two plots correspond directly with listings in Tables 5.1 - 5.2 and Tables 5.3 - 5.4, respectively, for Non-OB stars. O and B spectral type stars are typically at a much further distance than other stars. They typically showed multiple interstellar components from unique clouds at different velocities. Tables 5.3 5.11 provide a listing of the targets investigated and represent four categories: non-OB absorption, lack of absorption, and inconclusive results and then the OB targets. The tables provide the target name, the right ascension and declination (J2000) position, spectral classification, and distance (from parallax measurements). This data was retrieved directly from the SIMBAD database using the Simbad module from SLiPy. The author makes no claim of particular accuracy or uncertainty in these quantities and are provided by publications cited by SIMBAD.

\footnotetext{
${ }^{1}$ The mosaic used in the background of Figures 5.1 - 5.3 was adapted from data retieved from sky-map.org. Originally, the author attempted to construct the mosaics manually using the Montage image mosaicing software (montage.ipac.caltech.edu). A high-level wrapper to the Montage software was developed to facilitate the creation of large mosaics using DSS, SDSS, and 2MASS data (or user provided data). This functionality is provided within SLiPy under the Montage module (see Appendix C.13). Its usage is not documented here. Ultimately, there were serious issues with the state of the data retrieved from the DSS survey. sky-map.org has used the same data from DSS and put together a quality mosaic that has overcome many of these challenges. In the interest of efficiency, the sky-map data was used instead and converted to FITS format to mark the targets using APLpy (aplpy.github.io).
} 


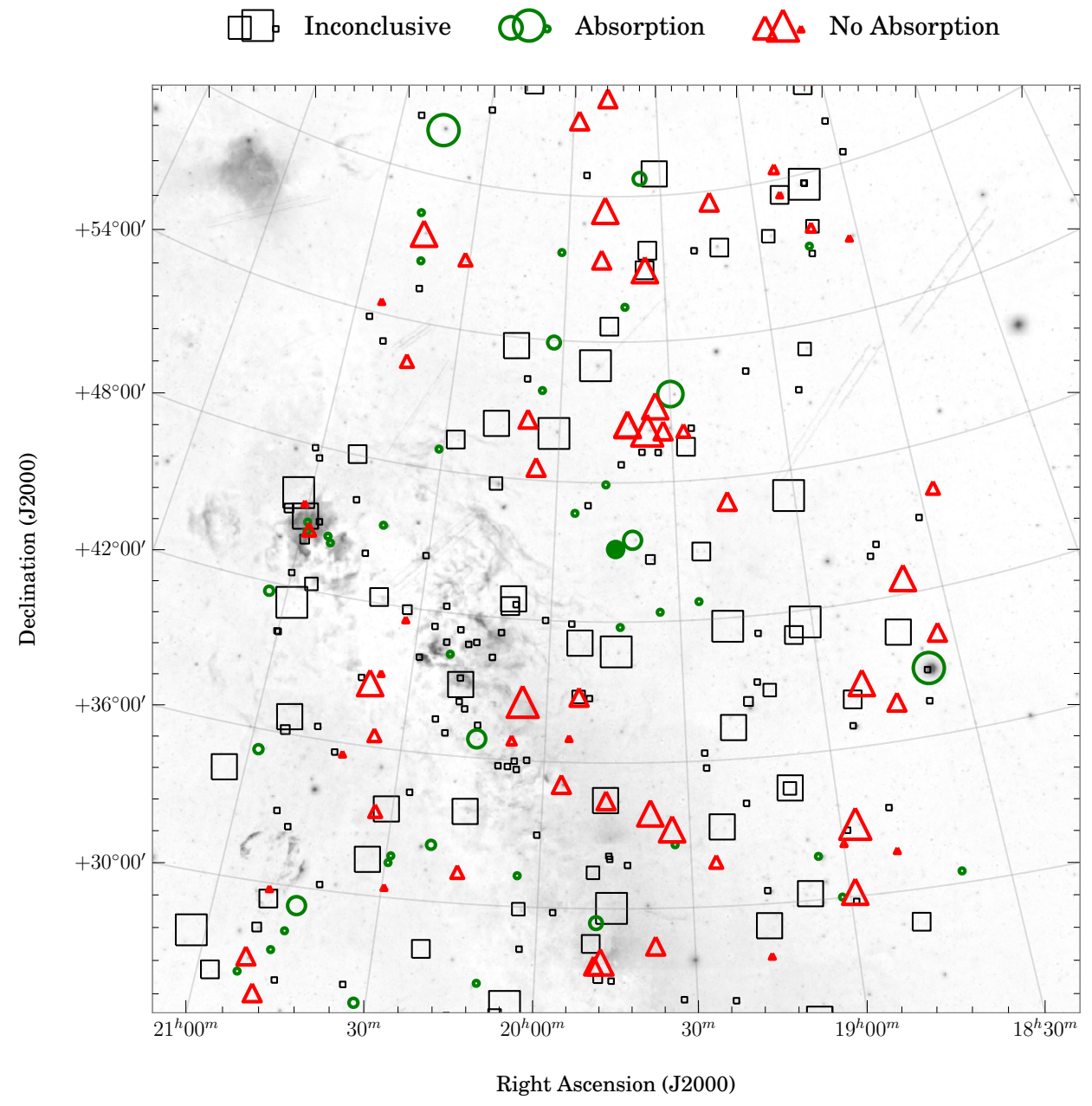

Figure 5.1: The above figure shows the entire $40^{\circ}$ square field with every target star in the study represented. There are three different marker styles. The positive identification of interstellar sodium absorption is represented by a green circle. The definitive absence of absorption is given by a red triangle. The remaining data found to be inconclusive is given as a black square. $\delta$ Cygni is located in the center of the field and is marked by a filled-in green circle. The sizes of the markers are inversely proportional to the distance to that star. The largest markers show stars within the Local Cavity and the smallest markers are from $100 \mathrm{pc}$ to more than $1 \mathrm{kpc}$. 


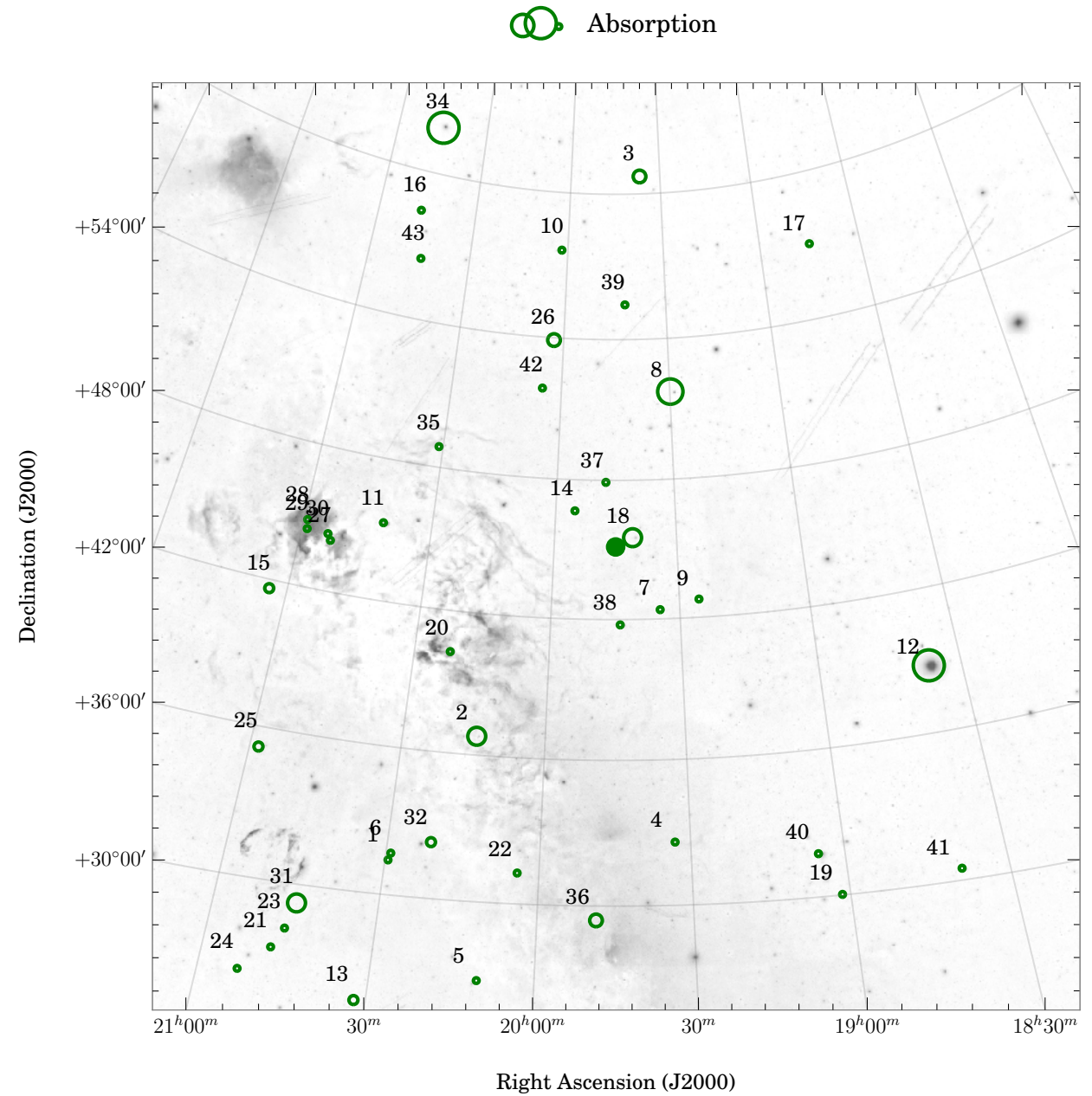

Figure 5.2: The above figure includes those targets from Figure 5.1 that have been identified as containing measurable interstellar $\mathrm{Na}$ I absorption. The annotations for the markers correspond to the items in Tables 5.1 and 5.2. Targets 12 and 34 are marked as measurable but have since been rendered inconclusive. 


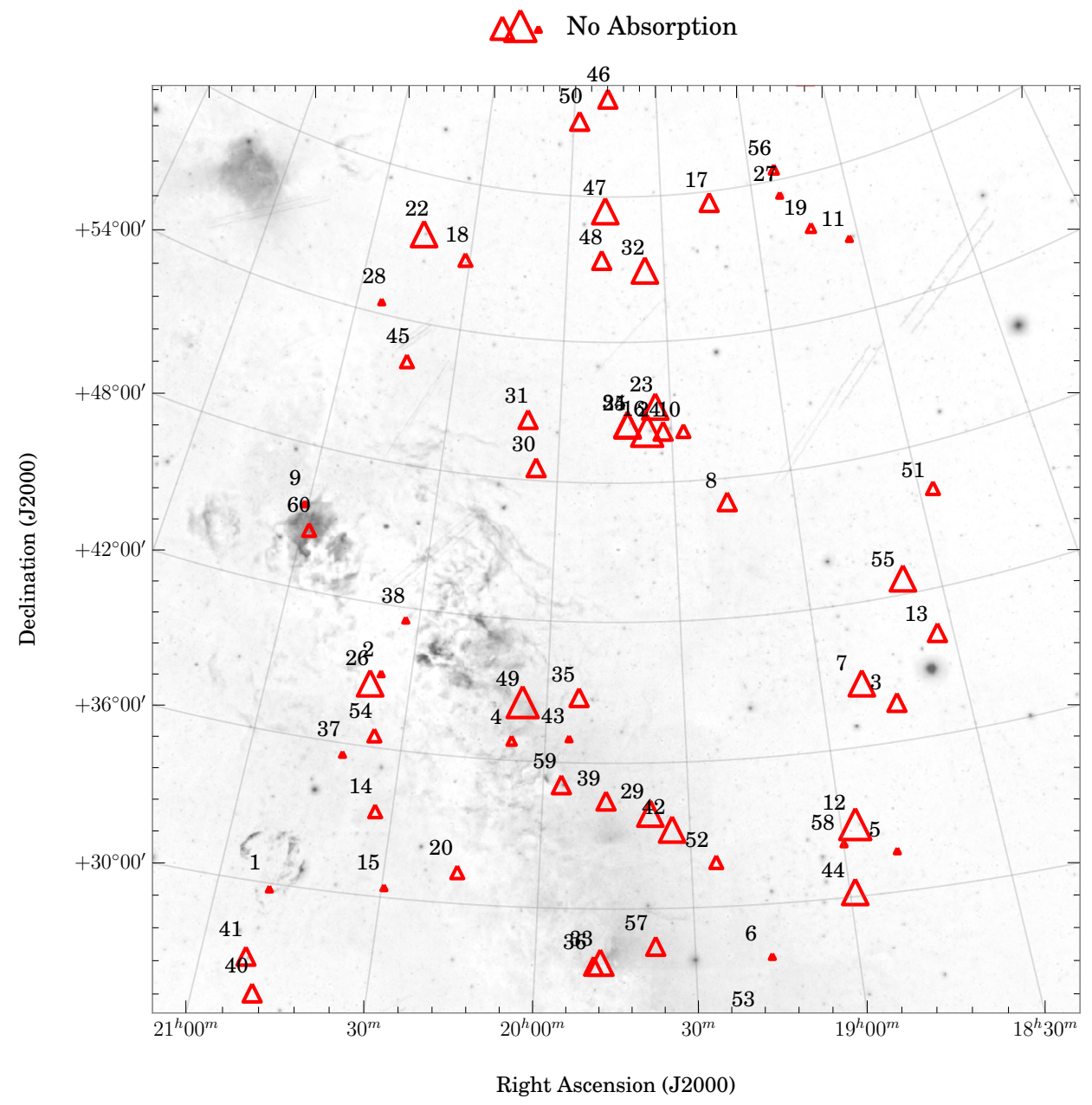

Figure 5.3: The above figure includes those targets from Figure 5.1 that have been identified as containing definitively no interstellar $\mathrm{Na}$ I absorption. The annotations for the markers correspond to the items in Tables 5.3 and 5.4. 
TABLE 5.1

Non-OB Targets with Measurable Absorption - A

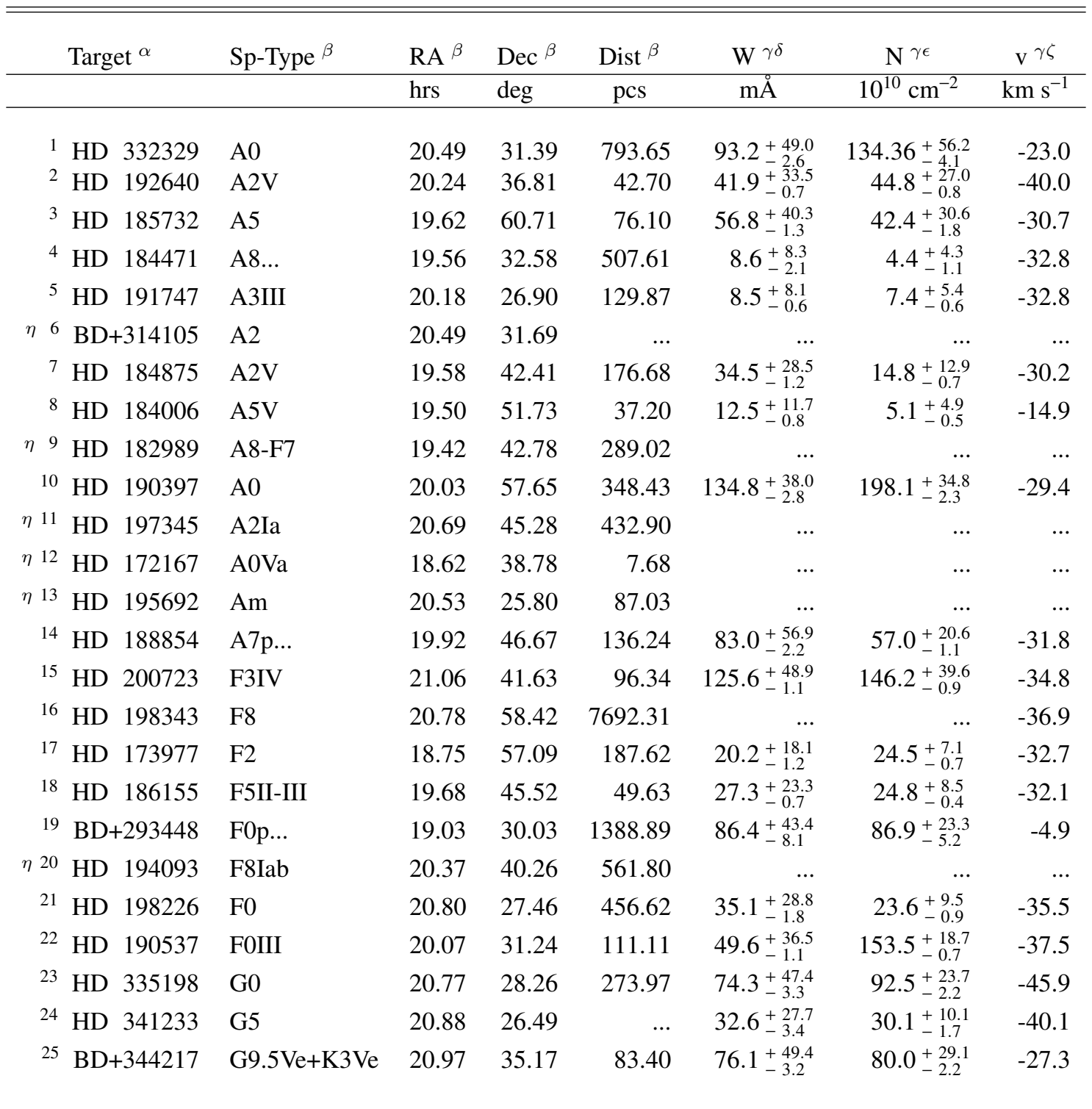

${ }^{\alpha}$ Numbers correspond to annotations in Figure 5.2.

${ }^{\beta}$ Retrieved from SIMBAD database (as of June 13, 2015); uncertainties not listed.

${ }^{\gamma}$ Measurements and uncertainties computed with Profile module.

${ }^{\delta}$ Equivalent Width for Na I D2.

${ }^{\epsilon}$ Corrected Column Density for Na I line, see text (Savage and Sembach, 1991).

$\zeta$ Velocity of Na I D2 line center, from 5,889.951 $\AA$. Uncertainty is approximately $2.6 \mathrm{~km} \mathrm{~s}^{-1}$.

${ }^{\eta}$ Inconclusive identification. 


\section{TABLE 5.2}

Non-OB Targets with Measurable Absorption - B

\begin{tabular}{|c|c|c|c|c|c|c|c|c|}
\hline \multirow{2}{*}{\multicolumn{2}{|c|}{ Target $^{\alpha}$}} & Sp-Type ${ }^{\beta}$ & $\mathrm{RA}^{\beta}$ & $\operatorname{Dec}^{\beta}$ & Dist ${ }^{\beta}$ & $\mathrm{W}^{\gamma \delta}$ & $\mathrm{N}^{\gamma \epsilon}$ & $\mathrm{v}^{\gamma \zeta}$ \\
\hline & & & hrs & deg & pcs & $\mathrm{m} \AA$ & $10^{10} \mathrm{~cm}^{-2}$ & $\mathrm{~km} \mathrm{~s}^{-1}$ \\
\hline 26 & HD 190508 & G5 & 20.04 & 53.89 & 61.96 & $89.5_{-5.5}^{+47.6}$ & $190.6_{-3.9}^{+28.7}$ & -24.8 \\
\hline$\theta 27$ & $\mathrm{BD}+433754$ & G5V & 20.88 & 44.12 & $\ldots$ & $209.9_{-6.7}^{+41.9}$ & $\begin{array}{r}-3.9 \\
\ldots\end{array}$ & -26.4 \\
\hline$\theta 28$ & HD 200019 & G5 & 20.99 & 44.79 & 204.50 & $113.4_{-2.4}^{+59.7}$ & $\ldots$ & -39.8 \\
\hline 29 & HD 199939 & G9III:... & 20.98 & 44.41 & 306.75 & $\begin{array}{l}87.9_{-4.2}^{+}+46.8 \\
\end{array}$ & $89.3_{-2.8}^{+24.6}$ & -38.4 \\
\hline 30 & HD 199178 & G5III-IV & 20.90 & 44.39 & 101.32 & $11.5_{-1.1}^{+10.8}$ & $12.9_{-0.6}^{+4.0}$ & -4.3 \\
\hline 31 & HD 335070 & K0 & 20.75 & 29.27 & 57.41 & $53.8_{-3.0}^{+39.4}$ & $34.8_{-1.6}^{+13.9}$ & -32.3 \\
\hline$\eta 32$ & HD 193891 & K0 & 20.36 & 32.31 & 89.13 & $\begin{array}{l}126.9_{-2.2}^{+49.0}\end{array}$ & $\begin{array}{c}170.1_{-1.8}^{+37.5}\end{array}$ & -40.3 \\
\hline 33 & HD 170527 & K0 & 18.42 & 64.84 & 158.48 & $44.0_{-0.8}^{+33.6}$ & $56.6_{-0.5}^{+14.7}$ & -14.0 \\
\hline$\eta 34$ & HD 198149 & KOIV & 20.75 & 61.84 & 14.27 & $\ldots$ & $\ldots$ & $\cdots$ \\
\hline$\theta 35$ & $\mathrm{BD}+483149$ & K0 & 20.52 & 48.87 & $\ldots$ & $48.5_{-2.0}^{+36.1}$ & $\ldots$ & -24.9 \\
\hline$\theta 36$ & HD 332698 & K0 & 19.81 & 29.41 & 61.20 & $67.8_{-3.7}^{+43.6}$ & $\ldots$ & -7.3 \\
\hline$\theta 37$ & HD 187372 & M1III & 19.79 & 47.91 & 299.40 & $48.5_{-2.3}^{+36.0}$ & $\ldots$ & -31.6 \\
\hline 38 & HD 186619 & MOIII & 19.73 & 41.77 & 174.83 & $62.6_{-2.8}^{+42.5}$ & $175.6_{-1.8}^{+22.8}$ & -38.1 \\
\hline$\eta 39$ & HD 186532 & M5IIIa & 19.70 & 55.46 & 316.46 & $\ldots$ & $\ldots$ & $\ldots$ \\
\hline$\eta 40$ & HD 177808 & M0III & 19.08 & 31.74 & 189.75 & $\ldots$ & $\ldots$ & -32.9 \\
\hline$\theta 41$ & HD 172381 & M2III & 18.64 & 30.45 & 270.27 & $118.1_{-14.2}^{+37.7}$ & $\ldots$ & -23.0 \\
\hline 42 & HD 190964 & M1IIIa & 20.09 & 51.84 & 219.30 & $\begin{array}{l}87.8_{-3.2}^{+47.0} \\
{ }^{2}\end{array}$ & $233.5_{-2.3}^{+29.0}$ & -30.7 \\
\hline 43 & HD 197939 & M3III & 20.74 & 56.49 & 336.70 & $123.7_{-2.9}^{+37.9}$ & $175.2_{-2.2}^{+32.4}$ & -30.0 \\
\hline
\end{tabular}

${ }^{\alpha}$ Numbers correspond to annotations in Figure 5.2.

${ }^{\beta}$ Retrieved from SIMBAD database (as of June 13, 2015); uncertainties not listed.

$\gamma$ Measurements and uncertainties computed with Profile module.

${ }^{\delta}$ Equivalent Width for Na I D2.

${ }^{\epsilon}$ Corrected Column Density for Na I line, see text (Savage and Sembach, 1991).

$\zeta$ Velocity of Na I D2 line center, from 5,889.951 $\AA$. Uncertainty is approximately $2.6 \mathrm{~km} \mathrm{~s}^{-1}$.

$\eta$ Inconclusive identification.

${ }^{\theta}$ Too Saturated to apply optical depth method. 
TABLE 5.3

Non-OB Targets with No Absorption - A

\begin{tabular}{|c|c|c|c|c|}
\hline Target ${ }^{\alpha}$ & Sp-Type ${ }^{\beta}$ & $\mathrm{RA}^{\beta}$ & $\operatorname{Dec}^{\beta}$ & Dist ${ }^{\beta}$ \\
\hline & & $h r s$ & deg & $p c s$ \\
\hline 1 HD 335238 & A2 & 20.85 & 29.80 & 952.38 \\
\hline${ }^{2}$ HD 196542 & Ap... & 20.61 & 39.10 & \\
\hline${ }^{3}$ HD 173648 & $\mathrm{Am}$ & 18.75 & 37.61 & 47.87 \\
\hline${ }^{4}$ HD 191158 & $\mathrm{Am}$ & 20.12 & 36.83 & 88.11 \\
\hline${ }^{5}$ HD 174567 & A0Vs & 18.83 & 31.63 & 278.55 \\
\hline${ }^{6}$ HD 180583 & F6Ib-II & 19.27 & 27.93 & 429.18 \\
\hline${ }^{7}$ HD 174912 & F8 & 18.86 & 38.63 & 30.56 \\
\hline${ }^{8}$ HD 181096 & F6IV: & 19.28 & 47.00 & 42.03 \\
\hline $9 \mathrm{BD}+443670$ & F8 & 21.01 & 45.50 & $\ldots$ \\
\hline${ }^{10}$ HD 183361 & F5 & 19.45 & 50.15 & 74.57 \\
\hline${ }^{11}$ HD 171635 & $\mathrm{~F} 7 \mathrm{Ib}$ & 18.54 & 57.05 & 649.35 \\
\hline${ }^{12} \mathrm{HD} 176051$ & $\mathrm{~F} 9 \mathrm{~V}+\mathrm{K} 1 \mathrm{~V}$ & 18.95 & 32.90 & 14.87 \\
\hline${ }^{13}$ HD 171485 & F5 & 18.55 & 40.16 & 41.70 \\
\hline${ }^{14}$ HD 195992 & F5 & 20.56 & 33.39 & 62.54 \\
\hline 15 HD 195295 & F5Iab: & 20.49 & 30.37 & 235.85 \\
\hline 16 HD 185395 & $\mathrm{~F} 3+\mathrm{V}$ & 19.61 & 50.22 & 18.34 \\
\hline 17 HD 180712 & F8 & 19.23 & 59.55 & 44.56 \\
\hline 18 HD 195872 & F8 & 20.52 & 56.89 & 66.31 \\
\hline${ }^{19}$ HD 173700 & F8 & 18.72 & 57.82 & 85.40 \\
\hline${ }^{20}$ HD 192804 & F8V & 20.26 & 31.24 & 76.22 \\
\hline${ }^{21}$ HD 172323 & F9V & 18.59 & 63.70 & 43.65 \\
\hline${ }^{22}$ HD 198084 & F8IV-V+F9IV-V & 20.76 & 57.58 & 27.29 \\
\hline${ }^{23}$ HD 184960 & F7V & 19.57 & 51.24 & 25.11 \\
\hline${ }^{24}$ HD 184448 & F8 & 19.53 & 50.18 & 50.63 \\
\hline${ }^{25}$ HD 186408 & $\mathrm{G} 1.5 \mathrm{Vb}$ & 19.70 & 50.53 & 21.08 \\
\hline${ }^{26}$ HD 196850 & G1V & 20.64 & 38.64 & 27.16 \\
\hline 27 HD 175306 & G9III_Fe-0.5 & 18.85 & 59.39 & 104.82 \\
\hline${ }^{28}$ HD 199191 & GIII+... & 20.89 & 54.52 & 151.06 \\
\hline${ }^{29}$ HD 185501 & G5 & 19.63 & 33.89 & 31.96 \\
\hline${ }^{30}$ HD 191022 & G0 & 20.09 & 48.57 & 50.33 \\
\hline
\end{tabular}

${ }^{\alpha}$ Numbers correspond to annotations in Figure 5.3.

${ }^{\beta}$ Retrieved from SIMBAD database (as of June 13, 2015); uncertainties not listed. 
TABLE 5.4

Non-OB Targets with No Absorption - B

\begin{tabular}{|c|c|c|c|c|c|}
\hline \multirow{2}{*}{\multicolumn{2}{|c|}{ Target $^{\alpha}$}} & Sp-Type ${ }^{\beta}$ & $\mathrm{RA}^{\beta}$ & $\operatorname{Dec}^{\beta}$ & Dist $^{\beta}$ \\
\hline & & & hrs & deg & $p c s$ \\
\hline 31 & HD 191649 & G0 & 20.15 & 50.59 & 45.27 \\
\hline 32 & HD 185414 & G0 & 19.60 & 56.98 & 24.11 \\
\hline 33 & HD 187237 & G2IV-V & 19.80 & 27.87 & 26.24 \\
\hline 34 & HD 186427 & G3V & 19.70 & 50.52 & 21.21 \\
\hline 35 & HD 188326 & G8IVv & 19.88 & 38.77 & 55.93 \\
\hline 36 & HD 187462 & G0IV & 19.82 & 27.73 & 50.38 \\
\hline 37 & HD 197310 & G0 & 20.69 & 35.55 & $\ldots$ \\
\hline 38 & HD 195988 & G0 & 20.55 & 41.47 & $\ldots$ \\
\hline 39 & HD 187123 & G5 & 19.78 & 34.42 & 48.26 \\
\hline 40 & HD 198483 & G0V & 20.83 & 25.77 & 51.23 \\
\hline 41 & HD 198809 & G7III & 20.87 & 27.10 & 57.80 \\
\hline 42 & HD 184499 & G0V & 19.56 & 33.20 & 31.92 \\
\hline 43 & HD 188650 & G1Ib-IIe... & 19.91 & 37.00 & 369.00 \\
\hline 44 & HD 176377 & G1V & 18.98 & 30.18 & 23.84 \\
\hline 45 & HD 197666 & G0 & 20.72 & 52.30 & 79.49 \\
\hline 46 & HD 187792 & G0 & 19.80 & 63.86 & 55.22 \\
\hline 47 & HD 187748 & G0 & 19.80 & 59.42 & 27.71 \\
\hline 48 & HD 187876 & G0 & 19.82 & 57.41 & 46.93 \\
\hline 49 & HD 190771 & G2V & 20.09 & 38.48 & 18.79 \\
\hline 50 & HD 189749 & G & 19.96 & 62.96 & 56.98 \\
\hline 51 & HD 170357 & G1V & 18.44 & 46.08 & 71.99 \\
\hline 52 & HD 182758 & G0 & 19.42 & 31.80 & 61.46 \\
\hline 53 & HD 181047 & G8V & 19.30 & 25.37 & 46.82 \\
\hline 54 & HD 196361 & G5 & 20.59 & 36.47 & 66.40 \\
\hline 55 & HD 172393 & G5 & 18.63 & 42.67 & 28.08 \\
\hline 56 & HD 175441 & G0 & 18.86 & 60.43 & 87.41 \\
\hline 57 & HD 185269 & G0IV & 19.62 & 28.50 & 50.28 \\
\hline 58 & HD 176670 & K2.5III & 19.00 & 32.15 & 338.98 \\
\hline 59 & HD 188947 & K0III & 19.94 & 35.08 & 41.37 \\
\hline 60 & HD 199870 & K0IIIbCN... & 20.97 & 44.47 & 79.30 \\
\hline
\end{tabular}

${ }^{\alpha}$ Numbers correspond to annotations in Figure 5.3.

${ }^{\beta}$ Retrieved from SIMBAD database (as of June 13, 2015); uncertainties not listed. 
TABLE 5.5

Non-OB Targets Inconclusive - A

\begin{tabular}{|c|c|c|c|c|}
\hline Target & Sp-Type ${ }^{\gamma}$ & $\mathrm{RA}^{\gamma}$ & $\operatorname{Dec} \gamma$ & Dist $\gamma$ \\
\hline & & $h r s$ & $d e g$ & $p c s$ \\
\hline BD+293427 & А5p... & 18.99 & 29.80 & $\ldots$ \\
\hline HD 340577 & $\mathrm{~A} 3$ & 20.58 & 26.49 & 699.30 \\
\hline HD 200405 & $\mathrm{~A} 0 \mathrm{pSrCr}$ & 21.03 & 47.91 & 746.27 \\
\hline HD 191742 & A7p & 20.16 & 42.54 & 304.88 \\
\hline HD 192678 & $\mathrm{~A} 4 \mathrm{p}$ & 20.23 & 53.66 & 198.02 \\
\hline HD 195725 & A7III & 20.49 & 62.99 & 41.84 \\
\hline HD 177196 & A6IV & 19.02 & 46.93 & 37.44 \\
\hline HD 183534 & A1V & 19.46 & 52.32 & 107.53 \\
\hline $\mathrm{BD}+353616 \mathrm{~A}$ & F0 & 19.44 & 35.71 & \\
\hline HD 341037 & F0 & 20.78 & 26.36 & 3333.33 \\
\hline HD 197572 & F7Ib... & 20.72 & 35.59 & 1538.46 \\
\hline HD 198726 & $\mathrm{~F} 5 \mathrm{Ib}+\mathrm{A} 0.8 \mathrm{~V}$ & 20.86 & 28.25 & 369.00 \\
\hline $\mathrm{BD}+423935$ & F5.5Ib-G5Ib: & 21.00 & 42.60 & -2564.10 \\
\hline HD 238932 & F5 & 18.74 & 56.78 & 120.92 \\
\hline $\mathrm{BD}+423607$ & F3 & 20.15 & 42.87 & 82.58 \\
\hline HD 194951 & F1II & 20.45 & 34.33 & 1000.00 \\
\hline HD 331319 & F3Ib & 19.82 & 31.45 & \\
\hline HD 195069 & FOV: & 20.45 & 49.38 & 37.57 \\
\hline HD 193370 & F6Ib & 20.31 & 34.98 & 970.87 \\
\hline HD 200805 & F5Ib & 21.07 & 45.16 & 373.13 \\
\hline HD 178593 & F8 & 19.14 & 25.37 & 61.35 \\
\hline HD 188307 & F8 & 19.88 & 41.08 & 53.30 \\
\hline HD 187253 & F8 & 19.77 & 54.64 & 72.41 \\
\hline HD 185239 & F8 & 19.58 & 57.77 & 84.53 \\
\hline HD 175225 & G9IVa & 18.86 & 52.98 & 25.67 \\
\hline HD 173701 & G8V & 18.74 & 43.83 & 26.69 \\
\hline HD 177153 & G0 & 19.03 & 41.49 & 41.48 \\
\hline HD 185351 & G9IIIbCN... & 19.61 & 44.69 & 40.83 \\
\hline BD+413931 & G5 & 20.92 & 42.30 & 67.29 \\
\hline HD 175306 & G9III_Fe-0.5 & 18.85 & 59.39 & 104.82 \\
\hline
\end{tabular}

${ }^{\gamma}$ Retrieved from SIMBAD database (as of June 13, 2015); uncertainties not listed. 
TABLE 5.6

Non-OB Targets Inconclusive - B

\begin{tabular}{|c|c|c|c|c|c|}
\hline \multirow{2}{*}{\multicolumn{2}{|c|}{ Target }} & Sp-Type $\gamma$ & $\mathrm{RA}^{\gamma}$ & $\operatorname{Dec}^{\gamma}$ & Dist $\gamma$ \\
\hline & & & $h r s$ & $d e g$ & $p c s$ \\
\hline HD & 182488 & $\mathrm{G} 9+\mathrm{V}$ & 19.39 & 33.22 & 15.76 \\
\hline HD & 175900 & G5 & 18.91 & 51.31 & 80.97 \\
\hline HD & 193795 & G4IV & 20.35 & 28.11 & 70.67 \\
\hline HD & 200391 & G0V+G5V & 21.04 & 27.81 & 52.00 \\
\hline HD & 181655 & G5V & 19.33 & 37.33 & 25.39 \\
\hline $\mathrm{BD}+$ & +364301 & G0 & 20.89 & 36.70 & $\ldots$ \\
\hline HD & 179484 & G5V & 19.19 & 38.78 & 52.85 \\
\hline HD & 190360 & G7IV-V & 20.06 & 29.90 & 15.86 \\
\hline HD & 178911 & $\mathrm{G} 1 \mathrm{~V}+\mathrm{K} 1 \mathrm{~V}$ & 19.15 & 34.60 & 52.33 \\
\hline HD & 199598 & G0V & 20.96 & 26.41 & 31.63 \\
\hline HD & 173605 & G5 & 18.71 & 57.87 & 65.70 \\
\hline HD & 190228 & G5IV & 20.05 & 28.31 & 61.61 \\
\hline HD & 188326 & G8IVv & 19.88 & 38.77 & 55.93 \\
\hline HD & 187123 & G5 & 19.78 & 34.42 & 48.26 \\
\hline HD & 182736 & G8IV & 19.40 & 44.93 & 57.64 \\
\hline HD & 171242 & G0 & 18.53 & 45.01 & 61.92 \\
\hline HD & 185657 & G6V & 19.63 & 49.28 & 140.25 \\
\hline HD & 171008 & G5 & 18.47 & 60.44 & 79.30 \\
\hline HD & 193216 & G5 & 20.28 & 50.28 & 30.77 \\
\hline HD & 178911B & G5 & 19.15 & 34.60 & 42.59 \\
\hline HD & 200102 & G1Ib & 21.00 & 45.00 & 1250.00 \\
\hline HD & 190403 & G5Ib-II & 20.06 & 29.99 & 588.24 \\
\hline HD & 174104 & G0Ib & 18.80 & 28.72 & 1315.79 \\
\hline HD & 191010 & G3Ib & 20.11 & 25.69 & 518.13 \\
\hline HD & 180161 & G8V & 19.20 & 57.67 & 20.02 \\
\hline HD & 180683 & G0 & 19.26 & 38.38 & 64.52 \\
\hline HD & 197037 & G0 & 20.66 & 42.25 & 32.33 \\
\hline HD & 180502 & G0IV & 19.26 & 29.12 & 85.54 \\
\hline HD & 197488 & $\mathrm{G} 2 \mathrm{~V}$ & 20.71 & 45.82 & 54.14 \\
\hline HD & 175425 & G0 & 18.89 & 37.99 & 53.53 \\
\hline
\end{tabular}

${ }^{\gamma}$ Retrieved from SIMBAD database (as of June 13, 2015); uncertainties not listed. 
TABLE 5.7

Non-OB Targets Inconclusive - C

\begin{tabular}{rlllr}
\hline \hline Target & Sp-Type & & & \\
RA & & & \\
Dec & Dist \\
\hline & & hrs & deg & pcs \\
\hline HD 193215 & G5 & 20.26 & 64.20 & 68.97 \\
HD 172557 & G5 & 18.61 & 63.36 & 52.63 \\
HD 184601 & G0 & 19.53 & 60.86 & 83.68 \\
HD 199100 & G5IV-V & 20.89 & 36.13 & 57.60 \\
HD 195987 & G9V & 20.55 & 41.90 & 22.05 \\
HD 187548 & G0V & 19.82 & 28.61 & 45.11 \\
HD 177780 & G3V & 19.07 & 41.00 & 55.01 \\
HD 190605 & G2V & 20.08 & 26.05 & 44.98 \\
HD 178911 & G1V+K1V & 19.15 & 34.60 & 52.33 \\
HD 178450 & G8VSB & 19.13 & 30.25 & 27.96 \\
HD 173024 & G5 & 18.69 & 40.48 & 80.97 \\
HD 173289 & G5 & 18.71 & 44.27 & 93.81 \\
HD 197140 & G5 & 20.67 & 38.78 & 53.56 \\
HD 197207 & G5V & 20.69 & 30.19 & 55.46 \\
HD 189087 & K1V & 19.95 & 29.82 & 26.72 \\
HD 234677 & K4Ve+K7.5Ve & 18.57 & 51.72 & 16.35 \\
HD 197989 & K0III-IV & 20.77 & 33.97 & 22.29 \\
HD 183255 & K2.5V & 19.44 & 49.47 & 25.01 \\
HD 191026 & K0IV & 20.11 & 35.97 & 23.95 \\
HD 338867 & K0 & 19.81 & 27.29 & $\ldots$ \\
HD 199956 & K0 & 20.98 & 44.06 & 201.61 \\
HD 334514 & K0 & 20.56 & 31.47 & $\ldots$ \\
HD 199546 & K0 & 20.93 & 53.81 & 452.49 \\
HD 189806 & K2 & 20.01 & 32.95 & 189.75 \\
HD 181209 & K0 & 19.30 & 34.14 & 432.90 \\
HD 188056 & K3III & 19.84 & 52.99 & 62.07 \\
BD+333930 & K0V & 20.52 & 33.54 & 48.85 \\
BD+334140 & K0 & 21.06 & 34.22 & $\ldots$ \\
HD 192787 & K0III & 20.26 & 33.73 & 97.47 \\
HD 196134 & K0III-IV & 20.56 & 41.77 & 92.08 \\
& & & & \\
& & & & \\
\hline
\end{tabular}

${ }^{\gamma}$ Retrieved from SIMBAD database (as of June 13, 2015); uncertainties not listed. 
TABLE 5.8

Non-OB Targets Inconclusive - D

\begin{tabular}{|c|c|c|c|c|}
\hline Target & Sp-Type ${ }^{\gamma}$ & RA $^{\gamma}$ & $\operatorname{Dec}^{\gamma}$ & Dist $\gamma$ \\
\hline & & hrs & deg & $p c s$ \\
\hline HD 176408 & K1III & 18.95 & 57.81 & 84.67 \\
\hline HD 198550 & K5V & 20.84 & 29.38 & 20.99 \\
\hline HD 192910 & $\mathrm{~K} 3 \mathrm{Ib}+$ & 20.26 & 47.71 & 324.68 \\
\hline HD 193469 & K5Ib & 20.32 & 39.00 & 1492.54 \\
\hline HD 198794 & K3Ib & 20.85 & 48.03 & 232.02 \\
\hline HD 200560 & $\mathrm{~K} 2.5 \mathrm{~V}$ & 21.04 & 45.88 & 19.47 \\
\hline HD 190470 & K3V & 20.07 & 25.79 & 21.95 \\
\hline BD+413306 & K0V & 19.32 & 41.63 & 35.68 \\
\hline $\mathrm{BD}+522815$ & K7-V & 20.84 & 52.90 & 22.42 \\
\hline HD 332518 & K5V & 19.76 & 30.01 & 20.32 \\
\hline HD 331093 & K0 & 19.71 & 31.74 & 55.07 \\
\hline HD 191806 & K0 & 20.16 & 52.28 & 69.40 \\
\hline HD 171607 & K0 & 18.53 & 61.78 & 46.75 \\
\hline HD 179094 & K1IV & 19.14 & 52.43 & 71.02 \\
\hline HD 198425 & $\mathrm{~K} 2.5 \mathrm{~V}$ & 20.82 & 32.28 & 24.05 \\
\hline HD 188753 & K0 & 19.92 & 41.87 & 46.23 \\
\hline $\mathrm{BD}+400883$ & M3.0V & 21.00 & 40.07 & 15.29 \\
\hline HD 173739 & M3.0V & 18.71 & 59.63 & 3.57 \\
\hline GJ 0747 & M3V & 19.13 & 32.54 & 8.13 \\
\hline GJ 0809 & $\mathrm{M} 2.0 \mathrm{~V}$ & 20.89 & 62.15 & 7.05 \\
\hline HD 186686 & M2-7e & 19.73 & 48.78 & -3448.28 \\
\hline GJ 0725 & $\mathrm{M} 3.0 \mathrm{~V}$ & 18.71 & 59.63 & 3.57 \\
\hline $\mathrm{BD}+394208$ & M3Iab: & 20.48 & 39.98 & 1098.90 \\
\hline $\mathrm{BD}+364025$ & M4Iab & 20.36 & 36.93 & 133.69 \\
\hline HD 182190 & M1III & 19.34 & 57.65 & 242.13 \\
\hline HD 199871 & MOIII & 20.97 & 41.36 & 213.68 \\
\hline HD 186776 & M4III & 19.75 & 40.72 & 251.26 \\
\hline HD 175865 & M5III & 18.92 & 43.95 & 91.41 \\
\hline HD 175588 & M4II & 18.91 & 36.90 & 225.73 \\
\hline HD 184786 & M4.5III & 19.56 & 49.26 & 406.50 \\
\hline
\end{tabular}

${ }^{\gamma}$ Retrieved from SIMBAD database (as of June 13, 2015); uncertainties not listed. 
TABLE 5.9

Non-OB Targets Inconclusive - E

\begin{tabular}{|c|c|c|c|c|}
\hline Target & Sp-Type $\gamma$ & $\mathrm{RA}^{\gamma}$ & $\operatorname{Dec} \gamma$ & Dist $\gamma$ \\
\hline & & $h r s$ & deg & $p c s$ \\
\hline GJ 0815 & M3.0V & 21.00 & 40.07 & 15.29 \\
\hline GJ 0766 & $\mathrm{M} 4.5 \mathrm{~V}$ & 19.76 & 27.13 & 10.31 \\
\hline HD 189063 & M0III & 19.91 & 60.82 & 540.54 \\
\hline HD 178003 & MOIII & 19.10 & 29.92 & 446.43 \\
\hline HD 180450 & MOIII & 19.26 & 30.53 & 389.11 \\
\hline HD 179869 & M3III & 19.20 & 41.24 & 317.46 \\
\hline HD 187849 & M2III & 19.84 & 38.72 & 212.31 \\
\hline HD 190544 & M1III & 20.02 & 64.82 & 180.18 \\
\hline HD 199305 & $\mathrm{M} 2.0 \mathrm{~V}$ & 20.89 & 62.15 & 7.05 \\
\hline HD 173740 & $\mathrm{M} 3.5 \mathrm{~V}$ & 18.71 & 59.63 & 3.45 \\
\hline HD $331161^{\epsilon}$ & K5 & 19.77 & 32.02 & 13.61 \\
\hline HD $190163^{\epsilon}$ & M5.5-6.5e & 20.02 & 50.04 & 401.61 \\
\hline HD $182917^{\epsilon}$ & M7IIIab+Be & 19.41 & 50.24 & 242.72 \\
\hline GJ $0802 \epsilon$ & M5.0V & 20.72 & 55.35 & 16.67 \\
\hline
\end{tabular}

${ }^{\gamma}$ Retrieved from SIMBAD database (as of June 13, 2015); uncertainties not listed.

${ }^{\epsilon}$ Inconclusive because the spectrum was bad (see text). 
TABLE 5.10

OB Targets - A

\begin{tabular}{|c|c|c|c|c|c|c|c|}
\hline \multirow[t]{2}{*}{ Target } & Sp-Type ${ }^{\beta}$ & $\mathrm{RA}^{\beta}$ & $\operatorname{Dec}^{\beta}$ & Dist ${ }^{\beta}$ & $\mathrm{W}^{\gamma \delta}$ & $\mathbf{N}^{\gamma \epsilon}$ & $\mathrm{v} \gamma \zeta$ \\
\hline & & $h r s$ & deg & $p c s$ & $m \AA$ & $10^{10} \mathrm{~cm}^{-2}$ & $k m s^{-1}$ \\
\hline${ }^{\theta \eta} \mathrm{HD} 188209$ & O9.5Iab & 19.87 & 47.03 & 1098.90 & $212.0_{-30}^{+27.9}$ & & -24.9 \\
\hline${ }^{\eta}$ HD 198820 & B3III & 20.87 & 32.85 & 531.91 & $\begin{array}{l}92.8_{-1.5}^{+53.2} \\
{ }^{2}\end{array}$ & $88.4_{-1.0}^{+26.1}$ & -0.5 \\
\hline${ }^{\eta}$ HD 194335 & B2IIIe & 20.40 & 37.48 & 414.94 & $101.6_{-1.2}^{+46.5}$ & $\begin{array}{l}118.5_{-0.8}^{+28.2} \\
\text { (1) }\end{array}$ & -20.8 \\
\hline${ }^{\eta}$ HD 200120 & B1.5Vnne & 21.00 & 47.52 & 434.78 & $86.0_{-1.0}^{+46.4}$ & $94.8_{-0.7}^{+29.7}$ & -29.9 \\
\hline HD 182255 & B6III & 19.38 & 26.26 & 120.48 & $16.6_{-1.1}^{+15.4}$ & $11.6_{-0.6}^{+4.8}$ & -5.7 \\
\hline${ }^{\eta}$ HD 338529 & B5 & 19.54 & 26.39 & 137.93 & $40.2_{-1.9}^{+31.6}$ & $64.4_{-1.1}^{+14.2}$ & -24.4 \\
\hline${ }^{\eta} \mathrm{HD} 183056$ & B9sp... & 19.44 & 36.32 & 216.92 & $22.8_{-0.7}^{+20.0}$ & $13.6_{-0.4}^{+6.0}$ & -33.3 \\
\hline HD 174638 & B8II-IIIep & 18.83 & 33.36 & 294.99 & $164.2_{-2.4}^{+38.0}$ & $643.8_{-3.5}^{+48.4}$ & -17.5 \\
\hline${ }^{\eta} \mathrm{HD} 176437$ & B9III & 18.98 & 32.69 & 190.11 & $19.7_{-2.0}^{+17.6}$ & $\begin{array}{l}9.9_{-1.1}^{+4.7} \\
\end{array}$ & -34.0 \\
\hline HD 180163 & B2.5IV & 19.23 & 39.15 & 425.53 & $96.9_{-1.6}^{+46.6}$ & $109.8_{-1.1}^{+27.1}$ & -11.3 \\
\hline HD 192685 & B3V & 20.25 & 25.59 & 303.95 & $27.8_{-1.1}^{+23.7}$ & $14.9_{-0.6}^{+6.9}$ & -31.2 \\
\hline HD 198183 & B5V & 20.79 & 36.49 & 235.85 & $174.1_{-3.5}^{+70.6}$ & $244.2_{-2.6}^{+49.3}$ & -28.9 \\
\hline${ }^{\eta} \mathrm{BD}+404124$ & $\mathrm{~B} 2 \mathrm{Ve}$ & 20.34 & 41.36 & 79.49 & $142.9_{-3.1}^{+38.5}$ & $\begin{array}{l}152.7_{-2.4}^{+36.3} \\
\end{array}$ & -41.0 \\
\hline${ }^{\eta}$ HD 194279 & B2Ia & 20.39 & 40.76 & 1724 & $120.8_{-2.2}^{+41.5}$ & $\begin{array}{l}222.0_{-1.9}^{+33.2} \\
-\end{array}$ & -39.9 \\
\hline${ }^{\eta}$ HD 172324 & B9Ib & 18.63 & 37.43 & 1030.93 & $\begin{array}{l}77.5_{-1.6}^{+45.6} \\
\text { (1. }\end{array}$ & $80.3_{-1.0}^{+22.3}$ & -32.8 \\
\hline$\eta \mathrm{BD}+413731$ & B3n & 20.40 & 42.30 & 2222.22 & $\begin{array}{l}131.2_{-4.6}^{+36.9} \\
\end{array}$ & $\begin{array}{l}513.6_{-4.6}^{+35.0} \\
\end{array}$ & -38.5 \\
\hline${ }^{\theta \eta} \mathrm{HD} 193322$ & $\mathrm{O} 9 \mathrm{~V}$ & 20.30 & 40.73 & 595.24 & $190.6_{-3.8}^{+43.1}$ & $\ldots$ & -33.3 \\
\hline${ }^{\theta \eta} \mathrm{HD} 195592$ & O9.7Ia & 20.51 & 44.32 & 909.09 & $\begin{array}{l}38.3_{-1.6}^{+30.8} \\
{ }^{2}\end{array}$ & $\ldots$ & -27.5 \\
\hline${ }^{\theta \eta} \mathrm{HD} 190864$ & O6.5III(f) & 20.09 & 35.61 & 487.80 & 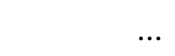 & $\ldots$ & $\ldots$ \\
\hline${ }^{\theta \eta} \mathrm{HD} 191423$ & ON9IIIn & 20.14 & 42.61 & 5263.16 & $239.1_{-4.1}^{+10.6}$ & $\ldots$ & -37.2 \\
\hline${ }^{\theta \eta} \mathrm{HD} 189957$ & O9.7III & 20.02 & 42.01 & -2702.70 & $\begin{array}{l}46.6_{-2.6}^{+34.8} \\
\end{array}$ & $\ldots$ & -38.3 \\
\hline${ }^{\theta \eta} \mathrm{HD} 192639$ & 7.5Iabf & 20.24 & 37.35 & 1886.79 & $\ldots$ & $\ldots$ & -30.3 \\
\hline${ }^{\theta \eta} \mathrm{HD} 191978$ & $\mathrm{O} 8 \mathrm{~V}$ & 20.18 & 41.35 & 100000.00 & $\cdots$ & $\ldots$ & -31.1 \\
\hline${ }^{\theta \eta}$ HD 190429 & O4If+O9.5II & 20.06 & 36.03 & 862.07 & $\ldots$ & $\ldots$ & -43.4 \\
\hline
\end{tabular}

${ }^{\beta}$ Retrieved from SIMBAD database (as of June 13, 2015); uncertainties not listed.

${ }^{\gamma}$ Measurements and uncertainties computed with Profile module.

${ }^{\delta}$ Equivalent Width for Na I D2.

${ }^{\epsilon}$ Corrected Column Density for Na I line, see text (Savage and Sembach, 1991).

$\zeta$ Velocity of Na I D2 line center, from 5895.924 A.

${ }^{\eta}$ Multiple components. Closest velocity listed.

${ }^{\theta}$ Too Saturated to apply optical depth method. 
TABLE 5.11

OB Targets $-\mathrm{B}$

\begin{tabular}{|c|c|c|c|c|c|c|c|}
\hline Target & Sp-Type ${ }^{\beta}$ & $\mathrm{RA}^{\beta}$ & $\operatorname{Dec}^{\beta}$ & $\operatorname{Dist}^{\beta}$ & $\mathrm{W}^{\gamma \delta}$ & $\mathrm{N}^{\gamma \epsilon}$ & $\mathrm{v} \gamma \zeta$ \\
\hline & & $h r s$ & deg & $p c s$ & $m \AA$ & $10^{10} \mathrm{~cm}^{-2}$ & $k m s^{-1}$ \\
\hline${ }^{\theta \eta} \mathrm{HD} 199579$ & O6.5V & 20.94 & 44.92 & 1666.67 & $\ldots$ & $\ldots$ & $\ldots$ \\
\hline${ }^{\theta \eta} \mathrm{HD} 193514$ & O7Ib & 20.32 & 39.27 & -925.93 & $\ldots$ & $\ldots$ & $\ldots$ \\
\hline$\theta \eta$ HD 190429 & O4If & 20.06 & 36.03 & $\ldots$ & $\ldots$ & $\ldots$ & ... \\
\hline$\theta \eta$ HD 186980 & O7.5III & 19.77 & 32.12 & -2631.58 & $\ldots$ & $\ldots$ & $\ldots$ \\
\hline${ }^{\theta \eta}$ HD 193443 & O9III & 20.31 & 38.28 & 1086.96 & $\ldots$ & $\ldots$ & $\ldots$ \\
\hline${ }^{\theta \eta} \mathrm{HD} 191612$ & O8fpe & 20.16 & 35.73 & 2040.82 & $\ldots$ & $\ldots$ & $\ldots$ \\
\hline${ }^{\theta \eta}$ HD 192281 & $\mathrm{O} 4.5 \mathrm{~V}(\mathrm{n})((\mathrm{f}))$ & 20.21 & 40.27 & 1265.82 & $\ldots$ & $\ldots$ & $\ldots$ \\
\hline${ }^{\theta \eta}$ HD 191201 & O9III+O9V & 20.12 & 35.72 & 628.93 & $\cdots$ & $\ldots$ & $\ldots$ \\
\hline$\theta \eta$ HD 193237 & B1-2_Ia-0_ep & 20.30 & 38.03 & 3125.00 & $\ldots$ & $\ldots$ & $\ldots$ \\
\hline${ }^{\theta \eta} \mathrm{HD} 198478$ & B4Ia & 20.82 & 46.11 & 714.29 & $\ldots$ & $\ldots$ & $\ldots$ \\
\hline${ }^{\theta \eta}$ HD 228712 & B0.5Ia & 20.28 & 40.89 & $\ldots$ & $\ldots$ & $\ldots$ & $\ldots$ \\
\hline${ }^{\theta \eta}$ HD 194839 & B0.5Iae & 20.44 & 41.38 & 1041.67 & $\ldots$ & $\ldots$ & $\ldots$ \\
\hline
\end{tabular}

${ }^{\beta}$ Retrieved from SIMBAD database (as of June 13, 2015); uncertainties not listed.

${ }^{\gamma}$ Measurements and uncertainties computed with Profile module.

${ }^{\delta}$ Equivalent Width for Na I D2.

${ }^{\epsilon}$ Corrected Column Density for Na I line, see text (Savage and Sembach, 1991).

$\zeta$ Velocity of Na I D2 line center, from $5895.924 \AA$.

${ }^{\eta}$ Multiple components. Closest velocity listed.

${ }^{\theta}$ Too Saturated to apply optical depth method. 


\section{CHAPTER 6}

\section{DISCUSSION}

\subsection{Structure and Target Selection Bias}

Looking at Figures 5.2 and 5.3 we can attempt to glean information about the possible structure of the LCCC. Using only the information from the positive absorption however is not appropriate because any apparent pattern in location here is primarily due to the fact that these were just the targets available from the archive (reflecting stars with potential exoplanets as studied by ELODIE) and not a uniform and/or unbiased selection pool of nearby stars. With that said, three stars of particular interest are HD 192640 (b03 Cyg, \#2, 42.7 pc), HD 184006 ( Cyg, \#8, 37.2 pc), and HD 186155 (\#18, 49.6 pc). These lines-of-sight show interstellar sodium absorption similar to that of $\delta$ Cyg (Welty et al., 1994) and are all within 50 parsecs, distances well inside the neutral gas "edge" of the local cavity. If we consider simultaneously the conclusions of the definitive absence of sodium in the spectra of all the stars represented in Figure 5.3, an imaginary line drawn

from b03 Cyg to $\iota$ Cyg (nearly 15 degrees across the sky) is interrupted by a clear lack of interstellar absorption in the intervening gaps (particularly just south of $\iota \mathrm{Cyg}$ ). This punctuated chain of absorption indicates the possibility of a similar broken ribbon structure to that of the LLCC. 


\subsection{The Soft X-ray Background}

As brought up in the Introduction, there is a soft X-ray background radiation (SXBR) believed to originate from the hot gas that fills the Local Bubble. Studies of the Local Leo Cold Cloud (Peek et al., 2011, Snowden et al., 2015) using the Rosat All Sky-Survey and have shown no shadowing effect despite the expectation that such shadowing should be present if the X-ray source were behind the cloud. Snowden et al. (2015) offers a concise synthesis of the constraints this places on a Local Hot Bubble given multi-wavelength observations of the LLCC and the current literature. Very recently, it has been shown that in fact the solar wind charge exchange (SWCX) contributes significantly to the SXBR at both $1 / 4$ and $3 / 4 \mathrm{keV}$. A lengthy discussion of the SXBR and the constraints it places on a Local Hot Bubble are not offered here, but indeed the LLCC has provided a near perfect test case for studying the dynamics and content of the Local Bubble. Unfortunately, it offers only a sample of one object. Now, this preliminary study of the LCCC provides needed observations to now begin multi-wavelength observations and extend the current understanding of the complex of local interstellar clouds.

\subsection{Stellar vs Interstellar}

I would like to make a brief comment in regards to the interpretation rendered on stellar lines versus interstellar lines. It can at times be difficult to immediately distinguish between weak stellar absorption and a real interstellar component. For the most part, the dominant sodium lines can be readily separated given their size. However, we can be fooled into a detection without making velocity comparisons. As an example, in Figure 6.1, I have over-plotted ${ }^{1}$ HD 181047 on top of HD 198149. It was originally

\footnotetext{
${ }^{1}$ Using .overplot() on a SPlot giving any other SPlot as an argument over plots the two. Any number of them can be given. The legend will automatically be updated provided they both have labels.
} 
suspected that HD 198149 might have an interstellar component, given its velocity. By over-plotting a similar spectrum we can see that even though the one was of a strength and velocity that make it a potential detection for the LCCC, it appears to be a weak stellar line. It moves with the other stellar lines - an interstellar line would not.

\subsection{Future Studies}

Follow up and additional observations of $\mathrm{Na}$ I for sight-lines in the Cygnus field using the KPNO Coudè Feed are needed to confirm results presented here. Preliminary results from the Coudè Feed have shown absorption of interstellar $\mathrm{Na}$ I towards seven stars, including $\delta$ Cygni. Six of the targets overlap with those studied from the ELODIE archive. The detections here are consistent with those measured with ELODIE. This data is available in Table 6.1. A similar campaign to that pursued for the Leo cloud over the past decade could add to the weight of current evidence in regards to our understanding of the local cavity. A proposal to use the Hubble Space Telescope for some of these much need observations was submitted. The author is a co-inverstigator on this proposal. The full text for this is provided in Appendix A. 


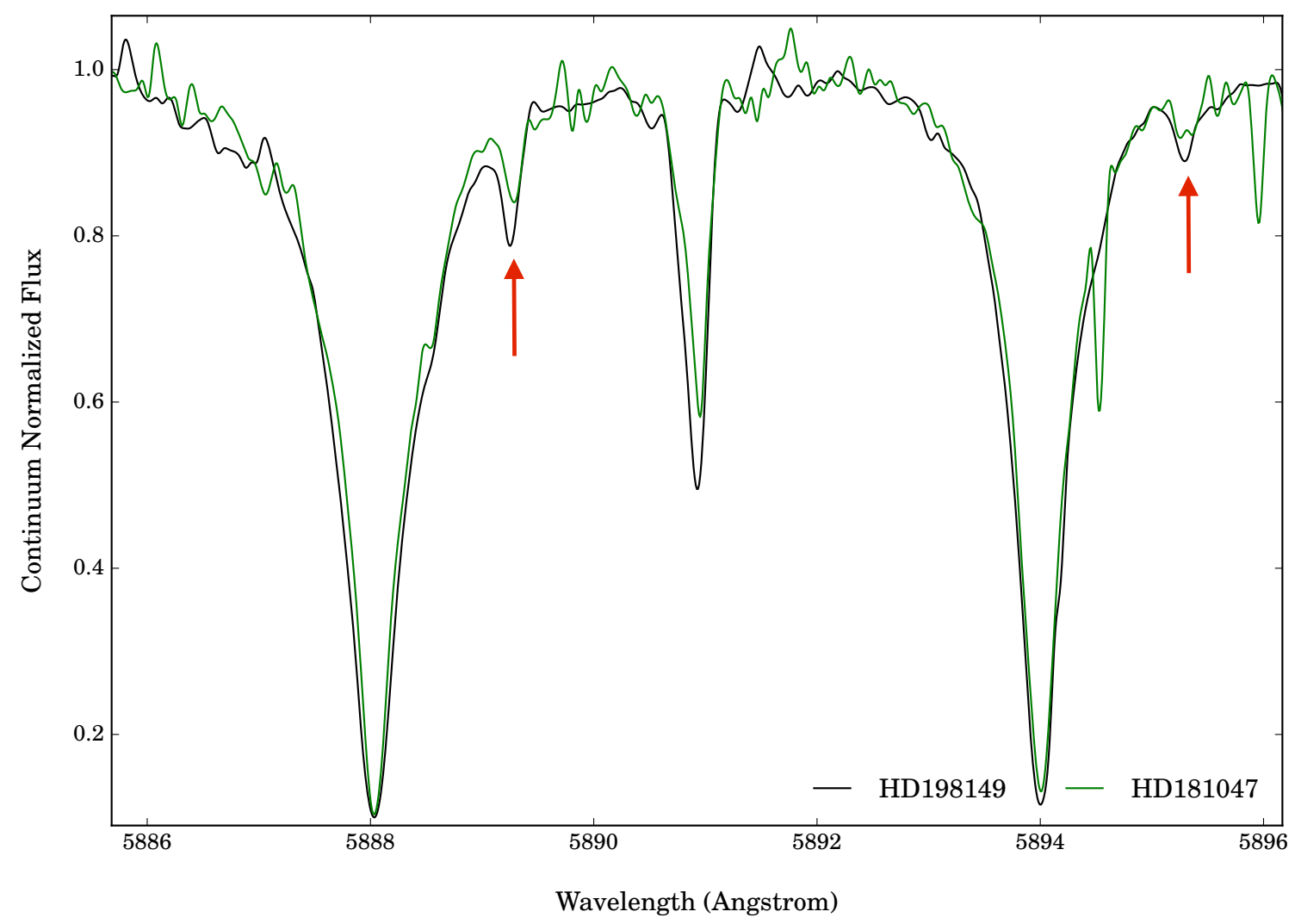

Figure 6.1: Often, narrow stellar lines can mimic interstellar absorption. An indication that a particular line may not be interstellar is when it not only appears in more than one spectrum of stars of the same type, but when after a velocity correction, it appears at the same shift from the larger component. In this figure, the spectra have been artificially shifted to overlay their lines for comparison. So while HD 198149 appears to have a narrow interstellar line and is at a velocity close to those for the cloud, it is more likely than not to in fact be stellar in origin. The D1 and D2 components in question are indicated by red arrows. 
TABLE 6.1

Stars Observed in the Region of $\delta$ Cygnus using the Coudè Feed

\begin{tabular}{|c|c|c|c|c|c|}
\hline Star & Spectral Type & $l^{\alpha}$ & $b^{\alpha}$ & Distance & Absorption \\
\hline & & (deg) & (deg) & (parsecs) & \\
\hline$\delta$ Cyg & B9.5IV & 78.71 & 10.24 & 52 & Y \\
\hline HD 175824 & FIII & 78.65 & 19.56 & 53 & $\mathrm{Y}$ \\
\hline HD 179958 & G4V & 80.67 & 17.27 & 22 & $\mathrm{~N}$ \\
\hline${ }^{\beta}$ HD 184006 & $\mathrm{~A} 5 \mathrm{Vn}$ & 83.61 & 15.44 & 38 & $\mathrm{~N}$ ? \\
\hline${ }^{\beta}$ HD 184960 & F7V & 83.46 & 14.59 & 26 & $\mathrm{~N}$ \\
\hline${ }^{\beta}$ HD 185351 & G9III & 77.62 & 11.35 & 41 & $\mathrm{Y}$ \\
\hline${ }^{\beta}$ HD 185395 & $\mathrm{~F} 4 \mathrm{~V}$ & 82.67 & 13.45 & 19 & $\mathrm{~N}$ \\
\hline${ }^{\beta}$ HD 186155 & F5II-III & 78.72 & 11.07 & 49 & $\mathrm{Y}$ \\
\hline HD 187160 & G0 & 78.16 & 9.60 & 43 & $\mathrm{Y}$ \\
\hline HD 192485 & F5V & 81.92 & 5.79 & 35 & $\mathrm{~N}$ \\
\hline${ }^{\beta}$ HD 192640 & $\mathrm{~A} 2 \mathrm{~V}$ & 74.45 & 1.17 & 41 & $\mathrm{Y}$ \\
\hline HD 192866 & A3 & 81.99 & 5.99 & 343 & $\mathrm{Y}$ \\
\hline
\end{tabular}




\section{CHAPTER 7}

\section{CONCLUSIONS}

The goal of this study was to search in the vicinity of $\delta$ Cygni for evidence of a new local interstellar cloud. Using public access archival data from ELODIE, we have indeed observed the absorption of interstellar sodium (D2 $\lambda 5889.591$ and D1 $\lambda 5895.924)$ extensively in the 40 degree square field here. The suspected stellar origin of the D2 line from Lallement et al. (2003) is now in question, given the presents of so much interstellar sodium in the region, particularly HD 186155 (target \#18 in Figure 5.2 and Table 5.1) in such close proximity to $\delta$ Cygni. These observations provide motivation for further investigation in the region. Our understanding of the Local Hot Bubble has been expanded by the observations and analysis of the Local Leo Cold Cloud and now the Local Cygnus Cold Cloud offers a new testbed for investigation.

In addition to the observations highlighted above, this work has made progress in other areas. First, it illustrates the potential for investigations of the interstellar medium using archival data provided by exoplanet studies. With the multitude of planetary search archives currently on line and coming in the near future, the amount of public access spectral data is growing and will facilitate "big data" absorption line studies for understanding the interstellar medium. With this comes the need for precision auto-calibration tools for larger spectral data sets. Indeed, at the moment one must still manually perform the fitting of individual lines; however, the reduction, preparation, and calibration of such data can be more quickly undertaken using the tools provided by 
SLiPy. Using this framework it may be possible to create automatic fitting procedures in the future.

As a platform independent, free, and open source product it is now open for further community development and may in time be rolled into the existing dominant package for computational astronomy with Python. The Spectrum class, built using numpy arrays, is an efficient structure for manipulating and working with spectral data. The Profile module gives the user a fitting and measurement facility for analyzing absorption features in the same environment within which they work with spectra as numerical objects (not just relying on file systems). The automated Telluric and Velocity corrections offer quick and precise calibrations.

The Telluric.Correct() function merits particular emphasis here. As described in Chapter 3, the method of using reference spectra to control for telluric absorption is effective in many instances. ${ }^{1}$ The all-in-one approach of the function offered here, to horizontally cross-correlate and vertical amplitude fit all the calibration spectra separately guarantees the best possible fit for the provided candidate reference spectra. With that, the ability to easily provide an arbitrary number of candidate reference spectra offers to automatically choose the best telluric reference out of all the candidates. In the region investigated in this study there exists a veritable forest of telluric lines. In the example control test plotted in Figure 3.2, immediately adjacent to the region shown, $\lambda \simeq 5810 \AA$, the signal to noise was observed to be $\simeq 320$. The mean $\mathrm{S} / \mathrm{N}$ in the same region for the six spectra of Regulus was $\simeq 310$. After the telluric correction procedure was applied, the signal to noise measured deep inside the afflicted region was $\simeq 210$. This is roughly a $\sqrt{2}$ reduction assuming the continuum would otherwise maintain a similar S/N up through $6000 \AA$. Therefore, we conclude that the algorithm has retained the expected $\mathrm{S} / \mathrm{N}$ for

\footnotetext{
${ }^{1}$ Recently, TelFit (Gullikson et al., 2014) was developed for Python and offers a wrapper to the Line-ByLine Radiative Transfer Model (LBLRTM). This is a separate model than that offered by Telluric.Correct().
} 
combining the two spectra and the reduction is a consequence of averaging two stochastic sets of noise. 


\section{REFERENCES}

L. Aller, G. Berge, R. Bless, S. Czyzak, L. Davis, H. Friedman, J. Greenberg, G. Haro, H. L. Johnson, H. Johnson, F. Kerr, W. Liller, B. Lynds, R. Minkowski, G. Münch, E. Parker, L. Spiterzer, and E. Upton. Nebula and interstellar matter. In B. M. Middlehurst and L. H. Aller, editors, Stars and Stellar Systems, volume VII. The University of Chicago Press, Chicago and London, 1968.

Astropy Collaboration, T. P. Robitaille, E. J. Tollerud, P. Greenfield, M. Droettboom, E. Bray, T. Aldcroft, M. Davis, A. Ginsburg, A. M. Price-Whelan, W. E. Kerzendorf, A. Conley, N. Crighton, K. Barbary, D. Muna, H. Ferguson, F. Grollier, M. M. Parikh, P. H. Nair, H. M. Unther, C. Deil, J. Woillez, S. Conseil, R. Kramer, J. E. H. Turner, L. Singer, R. Fox, B. A. Weaver, V. Zabalza, Z. I. Edwards, K. Azalee Bostroem, D. J. Burke, A. R. Casey, S. M. Crawford, N. Dencheva, J. Ely, T. Jenness, K. Labrie, P. L. Lim, F. Pierfederici, A. Pontzen, A. Ptak, B. Refsdal, M. Servillat, and O. Streicher. Astropy: A community Python package for astronomy. A\&A, 558:A33, Oct. 2013. doi: $10.1051 / 0004-6361 / 201322068$.

A. Baranne, D. Queloz, M. Mayor, G. Adrianzyk, G. Knispel, D. Kohler, D. Lacroix, J.-P. Meunier, G. Rimbaud, and A. Vin. ELODIE: A spectrograph for accurate radial velocity measurements. Astronomy and Astrophysics Supplement, 119:373-390, Oct. 1996.

F. Bouchy and Sophie Team. SOPHIE: the successor of the spectrograph ELODIE for extrasolar planet search and characterization. In L. Arnold, F. Bouchy, and C. Moutou, 
editors, Tenth Anniversary of 51 Peg-b: Status of and prospects for hot Jupiter studies, pages 319-325, Feb. 2006.

B. T. Draine. Physics of the Interstellar and Intergalactic Medium. Princeton University Press, Princeton and Oxford, 2011.

P. C. Frisch. The Local Bubble and Interstellar Material Near the Sun. Space Science Reviews, 130:355-365, June 2007. doi: 10.1007/s11214-007-9209-z.

K. Gullikson, S. Dodson-Robinson, and A. Kraus. Correcting for Telluric Absorption: Methods, Case Studies, and Release of the TelFit Code. AJ, 148:53, Sept. 2014. doi: $10.1088 / 0004-6256 / 148 / 3 / 53$.

J. D. Hunter. Matplotlib: A 2d graphics environment. Computing In Science \& Engineering, 9(3):90-95, 2007.

E. Jones, T. Oliphant, P. Peterson, et al. SciPy: Open source scientific tools for Python, 2001-. URL http://www. scipy.org/. [Online; accessed 2015-06-07].

A. Kramida, Yu. Ralchenko, J. Reader, and and NIST ASD Team. NIST Atomic Spectra Database (ver. 5.2), [Online]. Available: http://physics.nist.gov/asd [2015, July 13]. National Institute of Standards and Technology, Gaithersburg, MD., 2014.

R. Lallement, B. Y. Welsh, J. L. Vergely, F. Crifo, and D. Sfeir. 3D mapping of the dense interstellar gas around the Local Bubble. A\&A, 411:447-464, Dec. 2003. doi: 10.1051/0004-6361:20031214.

D. M. Meyer, J. T. Lauroesch, C. Heiles, J. E. G. Peek, and K. Engelhorn. A Cold Nearby Cloud inside the Local Bubble. ApJL, 650:L67-L70, Oct. 2006. doi: 10.1086/508658. 
D. M. Meyer, J. T. Lauroesch, J. E. G. Peek, and C. Heiles. The Remarkable High Pressure of the Local Leo Cold Cloud. ApJ, 752:119, June 2012. doi: 10.1088/0004-637X/752/2/119.

D. C. Morton. Atomic Data for Resonance Absorption Lines. III. Wavelengths Longward of the Lyman Limit for the Elements Hydrogen to Gallium. ApJS, 149:205-238, Nov. 2003. doi: 10.1086/377639.

J. Moultaka, S. A. Ilovaisky, P. Prugniel, and C. Soubiran. The ELODIE Archive. The Publications of the Astronomical Society of the Pacific, 116:693-698, July 2004. doi: $10.1086 / 422177$.

J. E. G. Peek, C. Heiles, K. M. G. Peek, D. M. Meyer, and J. T. Lauroesch. The Local Leo Cold Cloud and New Limits on a Local Hot Bubble. ApJ, 735:129, July 2011. doi: 10.1088/0004-637X/735/2/129.

P. Prugniel, C. Soubiran, M. Koleva, and D. Le Borgne. New release of the ELODIE library: Version 3.1. ArXiv Astrophysics e-prints, Mar. 2007.

B. D. Savage and K. R. Sembach. The analysis of apparent optical depth profiles for interstellar absorption lines. ApJ, 379:245-259, Sept. 1991. doi: 10.1086/170498.

S. L. Snowden, R. Egger, D. P. Finkbeiner, M. J. Freyberg, and P. P. Plucinsky. Progress on Establishing the Spatial Distribution of Material Responsible for the $14 \mathrm{keV}$ Soft X-Ray Diffuse Background Local and Halo Components. ApJ, 493:715-729, Jan. 1998. doi: $10.1086 / 305135$.

S. L. Snowden, C. Heiles, D. Koutroumpa, K. D. Kuntz, R. Lallement, D. McCammon, and J. E. G. Peek. Revisiting the Local Leo Cold Cloud and Revised Constraints on the Local Hot Bubble. ApJ, 806:119, June 2015. doi: 10.1088/0004-637X/806/1/119. 
L. Spitzer. Physical Processes in the Interstellar Medium. John Wiley \& Sons, Inc., 1978.

P. Stumpff. Two Self-Consistent FORTRAN Subroutines for the Computation of the Earth's Motion. A\&AS, 41:1, June 1980.

S. van der Walt, S. C. Colbert, and G. Varoquaux. The numpy array: A structure for efficient numerical computation, 2011.

G. L. Verschuur. Some Very Cold HI Clouds Found in Emission. ApL, 4:85-87, 1969.

S. S. Vogt, S. L. Allen, B. C. Bigelow, L. Bresee, B. Brown, T. Cantrall, A. Conrad, M. Couture, C. Delaney, H. W. Epps, D. Hilyard, D. F. Hilyard, E. Horn, N. Jern, D. Kanto, M. J. Keane, R. I. Kibrick, J. W. Lewis, J. Osborne, G. H. Pardeilhan, T. Pfister, T. Ricketts, L. B. Robinson, R. J. Stover, D. Tucker, J. Ward, and M. Z. Wei. HIRES: the high-resolution echelle spectrometer on the Keck 10-m Telescope. In D. L. Crawford and E. R. Craine, editors, Instrumentation in Astronomy VIII, volume 2198 of Society of Photo-Optical Instrumentation Engineers (SPIE) Conference Series, page 362, June 1994.

D. E. Welty, L. M. Hobbs, and V. P. Kulkarni. A high-resolution survey of interstellar NA I D1 lines. ApJ, 436:152-175, Nov. 1994. doi: 10.1086/174889.

M. Wenger, F. Ochsenbein, D. Egret, P. Dubois, F. Bonnarel, S. Borde, F. Genova, G. Jasniewicz, S. Laloë, S. Lesteven, and R. Monier. The SIMBAD astronomical database. The CDS reference database for astronomical objects. A\&AS, 143:9-22, Apr. 2000. doi: 10.1051/aas:2000332. 
APPENDIX A

\section{HUBBLE SPACE TELESCOPE PROPOSAL}




\title{
The Local Cygnus Cold Cloud - Testing the Hot Local Bubble
}

\author{
Scientific Category: ISM AND CIRCUMSTELLAR MATTER \\ Scientific Keywords: Dust, Galactic Structure, Interstellar And Intergalactic Medium \\ Instruments: STIS \\ Proprietary Period: 12 \\ Orbit Request \\ Cycle 23 \\ Proposal Size: Small \\ Prime \\ 5 \\ UV Initiative: Yes \\ Parallel
}

\begin{abstract}
There has recently been a revolution in our knowledge about the nature of clouds within the Local Bubble which is challenging our models for the content and structure of this gas. Over several decades a view had been developed of the Local Bubble as a cavity surrounding the Sun filled with hot ( 1E6 K) X-ray emitting gas that was devoid of cooler material. The recent detection of the Local Leo Cold Cloud withing the bubble has upset this view, and we are now trying to understand the formation of extremely cold gas $(20 \mathrm{~K})$ within this cavity. In order to further understand the formation and nature of this material, we request observations of two stars behind the Local Cygnus Cold Cloud, the second such pocket of cold material discovered in the Local Bubble. We will search for pressure variations within the cloud, which are expected to arise where turbulent fragmentation in the wake of collisions of warm clouds pushes the localized overpressures above the limiting values implied by the measured velocity constraints on the colliding clouds.
\end{abstract}




\section{J Lauroesch: The Local Cygnus Cold Cloud - Testing the Hot Local Bubble}

\section{Investigators:}

\begin{tabular}{llll} 
& Investigator & Institution & Country \\
\hline PI\& & J Lauroesch & University of Louisville Research Foundation, Inc. & USA/KY \\
CoI & G Lentner & University of Louisville Research Foundation, Inc. & USA/KY \\
\hline
\end{tabular}

Number of investigators: 2

\& Phase I contacts: 1

\section{Target Summary:}

\begin{tabular}{llll} 
Target & RA & Dec & Magnitude \\
\hline HR-7444 & 193441.2593 & +422445.04 & $\begin{array}{l}\text { V=5.348, 1.7e-11 at 1565 Ang, 2.4e-11 at 1965 Ang, } \\
1.8 \mathrm{e}-11 \text { at 2365 Ang }\end{array}$ \\
-B03-CYG & 201432.0333 & +364822.69 & $\begin{array}{l}\text { V=4.94 +/- 0.02, 3.5e-12 at 1598 Ang, 3e-11 at 2000 } \\
\text { Ang }\end{array}$ \\
\hline
\end{tabular}

\section{Observing Summary:}

\begin{tabular}{llll} 
Target & Config Mode and Spectral Elements & Flags & Orbits \\
\hline HR-7444 & STIS/FUV-MAMA Spectroscopic E140H & 2 \\
& & \\
& STIS98) & \\
& $(2113)$ & \\
-B03-CYG & STIS/FUV-MAMA Spectroscopic E140H & \\
& $(1598)$ & 3 \\
& STIS/NUV-MAMA Spectroscopic E230H \\
& $(2113)$ & \\
\hline
\end{tabular}

Total prime orbits: 5 


\section{Scientific Justification}

Our knowledge of the interstellar medium in the immediate Solar vicinity has been undergoing a revolution over the past few years. But even as we begin to have a more complete census of the interstellar components near the Sun, we find ourselves facing new difficulties in integrating the multi-wavelegth observations into a coherent whole. The limitations we face in our ability to model the interstellar medium near the Sun clearly reflect limitations in our ability to model the interstellar medium elsewhere in the Galaxy, for if we cannot understand the gas and dust near the Sun where we have all the advantages of proximity we have no hope of understanding what is going on in distant regions of the Galaxy. The physical conditions in the ambient interstellar medium surrounding the Sun also set the boundry conditions for the heliosphere, which is the edge of the Solar System (Frisch 1995).

From a combination of X-ray, UV and optical measurements of the interstellar medium a picture was developed over several decades of the interstellar environment within which the Solar System is embedded (Cox \& Reynolds 1987; Frisch 1995). Observations suggested the existence of a "Local Bubble" - a low density cavity surrounded by dense neutral material lying roughly in the galactic plane filled with hot gas with a temperature of more than one million K (Snowden et al. 1998) that was the source of the observed diffuse soft X-ray background emission. The neutral gas boundary of this region was marked by a "wall" of dense gas at distances between approximately 65 and $250 \mathrm{pc}$ of the Sun whose presence could be detected from strong $\mathrm{Na}$ I absorption detected in background stellar spectra of early-type stars (Sfeir et al. 1999; Lallement et al. 2003). More recently it was recognized that there were several warm $(\mathrm{T} \sim 10,000 \mathrm{~K})$ clouds in the bubble, and that the Solar System is itself embedded in a warm interstellar cloud. It was generally thought that there was no cold interstellar gas within this region of space (Lallement et al. 2003).

One interstellar cloud presented a puzzle for almost 3 decades. Originally identified by Verschuur (1969) as two roughly degree scale patches of very narrow H I $21 \mathrm{~cm}$ emission in the constellation Leo. This region was subsequently re-observed as part of the Millennium Arecibo $21 \mathrm{~cm}$ absorption line survey (Heiles \& Troland 2003). Coupling new observations of $21 \mathrm{~cm}$ absorption toward 3C 225 and 3C 237 with the Leiden-Dwingeloo $21 \mathrm{~cm}$ sky survey (Hartmann \& Burton 1997), Heiles \& Troland (2003) suggested that this cloud had a very thin, ribbon-like geometry. Motivated by these results, Meyer et al. (2006) obtained observations of the the interstellar Na I $\lambda 5889,5895 \AA$ absorption lines toward 33 stars at different distances in the direction of the Leo cloud using the Kitt Peak National Observatory $0.9 \mathrm{~m}$ coudé feed telescope. An extremely narrow absorption component was detected at the same velocity as the narrow $21 \mathrm{~cm}$ absorption detected toward 3C 225 in the spectra of 23 of the stars whose sky positions place them within the outer contour of the H I $21 \mathrm{~cm}$ emission. The closest of these stars had distances of $\sim 42$ parsecs, placing this cloud well within the neutral gas "edge" of the Local Bubble mapped by Lallement et al. (2003). While there were a number of warm, partially ionized clouds identified within the "Local Bubble" which was presumed to be full of hot gas (Redfield \& Linksky 2004; 2008), this was the first indication of cold material within this region. 
Peek et al. (2011) used optical observations of this Local Leo Cold Cloud (LLCC) to further constrain the distance to this material, placing it between 11 and 24.5 pc. Now such cold, neutral material will absorb X-ray emission, so if the soft X-rays observed around the Sun come from a pervasive hot gas filling the "Local Bubble" this cloud being well within the boundary should absorb the emission from the hot gas behind the cloud. In the direction of the LLCC the edge of the local cavity is between 100 and $150 \mathrm{pc}$ away (Meyer et al. 2006). An analysis of Rosat All Sky-Survey $1 / 4 \mathrm{keV}$ (C-band) data shows no shadowing of the X-ray flux in the direction of the LLCC, which suggests there is no diffuse, hot X-ray emitting bubble around the Sun (Peek et al. 2011). Instead Peek et al. (2011) suggested the source of the diffuse soft X-rays is charge exchange between the Solar Wind and the ambient interstellar medium (Cravens 1997; Cravens 2000; Wargelin et al. 2008). This is consistent with the model of Welsh \& Shelton (2009), who suggested that much of the observed X-ray flux was due to Solar wind exchange. However Galeazzi et al. 2014 suggested that no more than $40 \%$ of the diffuse X-ray emission could come from Solar Wind change exchange, and that the remainder must come from the surrounding interstellar medium. However these authors did not address the constraints from the LLCC. Further observations of cold clouds will place tight constraints on the location and origin of the diffuse X-rays.

Meyer et al. (2012) used Hubble Space Telescope observations of absorption by interstellar $\mathrm{C}$ I in the spectra stars behind this cloud to directly measure the pressure in this cloud. The pressures of $40,000-80,000 \mathrm{~cm}^{3} \mathrm{~K}$ are much greater than that of the warm clouds in the Local Bubble, where Redfield \& Linsky (2004) measured a mean thermal pressure to be $2300 \mathrm{~cm}^{3} \mathrm{~K}$ for 50 warm, partially ionized clouds within the Local Bubble. Measurements of the foreground X-ray emission from Solar Wind charge exchange (Robertson et al. 2010) and the Peek et al. (2011) finding of weak X-ray shadowing by the LLCC have weakened the case for a higher-pressure hot Local Bubble. Although some hot gas is undoubtedly present, its thermal pressure is most likely in a range $\left(3000-7000 \mathrm{~cm}^{3} \mathrm{~K}\right)$ consistent with that of the warm clouds (Frisch et al. 2011). Thus it is clear that the LLCC is not in thermal pressure equilibrium with either the hot gas or the warm clouds in the Local Bubble. That the LLCC is significantly overpressured with respect to the Local Bubble is qualitatively consistent with predictions its anomalously low temperature is the result of a warm cloud collision (Vazquez-Semadeni et al. 2006; Meyer et al. 2006; Redfield \& Linsky 2008). In their one-dimensional numerical simulations of the large-scale transonic (Mach number $\sim 1$ ) compression of colliding warm gas flows, Vazquez-Semadeni et al. (2006) find that a thin cold layer forms within the shocked interface. A common feature of the colliding warm flow models (Audit \& Hennebelle 2005; Gazol et al. 2005; Heitsch et al. 2006; Vazquez-Semadeni et al. 2006) is the turbulent fragmentation of the cold interface layer into clumpy structures. Some of the simulations (Audit \& Hennebelle 2005; Gazol et al. 2005) have shown that localized regions within these structures can reach pressures up to $10^{5} \mathrm{~cm}^{3} \mathrm{~K}$ even when the collision is transonic (Hennebelle et al. 2007). It is quite likely that the different LLCC pressures measured are due in part to real localized pressure variations, where the turbulent fragmentation expected in the wake of such collisions pushes the localized overpressures above the limiting values implied by the measured velocity constraints on the colliding clouds. 
In any case, the geometry and physical properties of such clouds provide constraints on theoretical models of their formation.

Currently there is only one published example of very cold material within the Local Bubble, the LLCC, and it is difficult to draw any significant conclusions for the general processes which lead to the formation of cold clouds from a sample of one object. Using the Kitt Peak National Observatory Coudé Feed telescope combined with archival optical and radio observations we have attempted to identify additional local cold clouds. The survey of Lallement et al. (2003) identified several anomalous sightlines within the Local Bubble which showed some possible interstellar Na I absorption, though it was typically somewhat below the limits representative of the "edge" of the Local Bubble. One sightline that drew immediate attention was that toward $\delta \mathrm{Cyg}$ - while it showed weak Na I absorption (an equivalent width of only $\sim 20 \mathrm{~m} \AA$, or about $\frac{1}{2}$ that seen toward the LLCC), it was notable for the narrowness of the absorption profile which shows hyper-fine splitting indicative of low temperatures and turbulent motions (Welty, Hobbs, \& Kulkarni 1989; Lallement et al. 2003). Jenkins (2002) used C I observations from HST/GHRS to measure a pressure in this cloud of $10^{3}<\mathrm{p} / \mathrm{k}<10^{4} \mathrm{~cm}^{-3} \mathrm{~K}$, more similar to that measured in the warm clouds in the Local Bubble. Using archival Elodie spectrograph observations we were able to confirm that there was a large region of Na I absorption in this region (Figure 1). This local Cygnus cold cloud (LCCC) thus represents a second of what may be a population of such cold material in the Solar vicinity (with other candidate clouds already known).

The question we seek to answer is does any of the material in the LCCC have the same high pressure as in the LLCC? The sightlines through the LLCC have a mean pressure of $60,000 \mathrm{~cm}^{-3} \mathrm{~K}$ Meyer et al. (2012), well in excess of the mean thermal pressure of 2300 $\mathrm{cm}^{-3} \mathrm{~K}$ for the warm, partially ionized clouds in the Local Bubble (Redfield \& Linsky 2004). Such excess pressures are expected in models that involve collisions of warm clouds (see, for example, Vazquez-Semadeni et al. 2006). Flow-driven formation of cold atomic and molecular clouds in models have shown that shocked flows of warm interstellar gas can provide a rapid formation of such clouds (Ballesteros-Paredes et al. 1999; Hennebelle \& Pérault 1999; Koyama \& Inutsuka 2000; Hartmann et al. 2001). While the timescales of instabilities in flow driven models of cold atomic and molecular cloud formation suggest that thermal instabilities drive their rapid formation (Heitsch, Hartmann \& Burkert 2008), and provide seeds for local gravitational collapse. Since this gas is significantly over-pressured one would expect it to have a relatively short lifetime, but a population of high pressure cold clouds argues for either some form of confinement or the rapid production of such clouds in the turbulent warm ISM. The measurent of a lower pressure toward $\delta$ Cyg (Jenkins 2002) suggests the gradual equillibration of such clouds with the ambient ISM, and support the idea that such clouds can become the seeds of the cold neutral medium. Pressure and density measurements in additional sightlines in the LCCC will determine if there is any variation in the pressure across the cloud, and the detection of high pressure gas like in the LLCC will point to turbulent fragmentation expected in the models. The measurement of the $\mathrm{H} \mathrm{I}$ column (inferred from the Zn II column) will enable us to use X-ray shadowing to place limits on the location and amount of hot gas in this direction. 


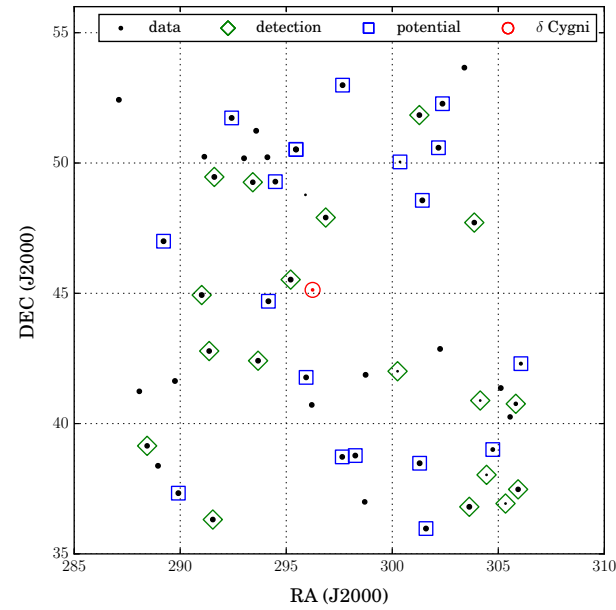

Figure 1: Positions of stars with detected Na I absorption behind the $\delta$ Cyg Cloud from observations taken from the Elodie spectrograph archive. The point size indicates the distance to the star, with small points showing distant stars and large points indicating nearby stars. Delta Cyg is marked by a circle.

\section{References}

Audit, E. \& Hennebelle, P. 2005, A\&A, 433, 1

Ballesteros-Paredes, J., Vázquez-Semadeni, E. \& Scalo, J. 1999, ApJ, 515, 286

Cox, D. P. \& Reynolds, R. J. 1987, ARA\&A, 25, 303

Cravens, T. E. 1997, GeoRL, 24, 105

Cravens, T. E. 2000, ApJL, 532, L153

Frisch, P. C. 1995, SSRv, 72, 499

Frisch, P. C., Redfield, S. \& Slavin, J. D. 2011, ARA\&A, 49, 237

Gazol, A., Vázquez-Semadeni, E. \& Kim, J. 2005, ApJ, 630, 911

Galeazzi, M., Chiao, M., Collier, M.R., Cravens, T., Kouttroumpa, D.. Lallement, R., Lepri,

S. T., McCammon, D., Morgan, K., Porter, F. S., Robertson, I. P., Snowden, S. L.,

Thomas, N. E., Uprety, Y., Ursino, E. \& Walsh, B. M. 2014, Nature, 512, 171

Hartmann, D. \& Burton, W. B. 1997, "Atlas of Galactic Neutral Hydrogen" Cambridge Univ. Press (Cambridge)

Hartmann, L., Ballesteros-Paredes, J, \& Bergin, E. A. 2001, ApJ, 562, 852

Heiles, C. \& Troland, T. H. 2003, ApJ, 586, 1067 

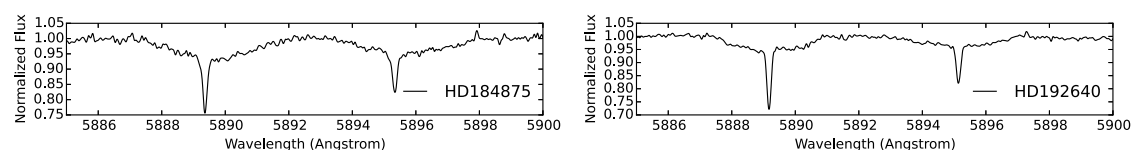

Figure 2: Archival Na I absorption spectra toward our selected stars behind the $\delta$ Cyg Cloud taken with the Elodie spectrograph

Heitsch, F., Slyz, A. D., Devriendt, J. E. G., Hartmann, L. W. \& Burkert, A. 2006, ApJ, 648, 1052

Heitsch, F., Hartmann, L. W. \& Burkert, A. 2008, ApJ, 683, 786

Hennebelle, P. \& Pérault, M. 1999, A\&A, 351, 309

Hennebelle, P., Audit, E. \& Miville-Deschênes, M.-A. 2007, A\&A, 465, 445

Jenkins, E. B. \& Tripp, T. M. 2001, ApJS, 137, 297

Jenkins, E. B. 2002, ApJ, 580, 938

Lallement, R., Welsh, B. Y., Vergely, J. L., Crifo, F. \& Sfeir, D. 2003, A\&A, 411, 447

Koyama, H. \& Inutsuka, S.-I. 2000, ApJ, 532, 980

Meyer, D. M., Lauroesch, J. T., Heiles, C., Peek, J. E. G. \& Engelhorn, K. 2006,ApJ, 650, L67

Meyer, D. M., Lauroesch, J. T., Peek, J. E. G. \& Heiles, C. 2012, ApJ, 752, 119

Peek, J. E. G., Heiles, C., Peek, K. M. G., Meyer, D. M., \& Lauroesch, J. T. 2011, ApJ, 735 , 129

Redfield, S. \& Linsky, J. L. 2004, ApJ, 613, 1004

Redfield, S. \& Linsky, J. L. 2008, ApJ, 673, 283

Robertson, I. P., Kuntz, K. D., Collier, M. R., Cravens, T. E. \& Snowden, S. L. 2010, AIPC, 1216, 532

Sfeir, D. M., Lallement, R., Crifo, F. \& Welsh, B. Y. 1999, A\&A, 346, 785

Snowden, S. L., Egger, R., Finkbeiner, D. P., Freyberg, M. J., \& Plucinsky, P. P. 1998, ApJ, $493, .715$

Vázquez-Semadeni, E., Ryu, D., Passot, T., González, R. F. \& Gazol, A. 2006, ApJ, 643, 245

Verschurr, G. L. 1969, Astrophys. Letters, 4, 85

Wargelin, B. J., Beiersdorfer, P., \& Brown, G. V. 2008, CaJPh, 86, 151

Welsh, B. Y. \& Shelton, R. L. 2009, Ap\&SS, 323, 1

Welty, D. E., Hobbs, L. M. \& Kulkarni, V. P. 1994, ApJ, 436, 152 


\section{Description of the Observations}

We have selected our targets from a sample of stars observed with the Kitt Peak Coudé Feed telescope or the Elodie spectrograph at OHP in the vicinity of $\delta C y g$ (Figure 1). Stars with suitably bright UV flux as well as rapid rotation were identified, and their $\mathrm{Na}$ I checked to be sure that the LCCC was detected but also that no more distant gas was seen (see Figure 2 ). For some of these stars we take advantage of one of the ND slits.

We target the C I fine structure lines as well as the Zn II absorption lines. By fitting the C I ground and fine-structure features as well as the (optical) hyper-fine split Na I absorption line one can determine the cloud temperature and turbulent velocity. Then the $\mathrm{C}$ I features allow one to determine the pressure and density within the cloud by using the excitation balance (Jenkins \& Tripp 2001). The Zn II lines serve as a proxy for H I since in the Cygnus region there is too much background gas to identify $\mathrm{H}$ I absorption or emission associated with the LCCC. From the observations we can determine a cloud thickness using the inferred $\mathrm{H}$ I column density and the volume density determined from $\mathrm{C}$ I. We can then infer a mass for the LCCC.

We will observe the strong $1560 \AA$ multiplet of C I we will using E140H centered at $1489 \AA$, and we will observe Zn II 2026, 2062 $\AA$ using E230H centered at $2113 \AA$. In addition

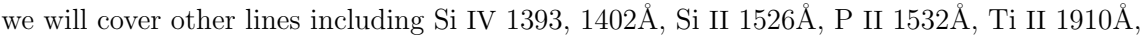
Cr II 2056, 2062, 2066 Åwhich will provide a measure of depletion and sample the warm/hot interstellar medium. We selected the $1560 \AA$ multiplet of C I due to the large exposure times needed toe cover the $1656 \AA$ mulitplet, and the lack of FUV flux in these objects preventing the use of multiplets such as 1277 or $1328 \AA$.

For HD 184875 we used the fluxes from the TD1 satellite, which are $1.7 \mathrm{e}-11$ at $1565 \AA$, $2.4 \mathrm{e}-11$ at $1965 \AA$, and $1.8 \mathrm{e}-11$ at $2365 \AA$. We used the highest of these fluxes to check bright object protection, and then the lower fluxes to estimate the exposure time. This results in exposure times of 1,150 seconds for the $1489 \AA$ setting using the $0.2 \mathrm{x} 0.09$ slit and 900 seconds at $2113 \AA$ using the $31 \mathrm{X} 0.05 \mathrm{NDA}$.

For HD 192640 low resolution, large aperture IUE SWP spectra show a flux at $1656 \AA$ of $6 \mathrm{E}-12 \mathrm{erg} / \mathrm{cm}^{2} / \mathrm{s} / \AA$, and a flux of no greater than $3 \mathrm{E}-11 \mathrm{erg} / \mathrm{cm}^{2} / \mathrm{s} / \AA$ anywhere in the $1763 \AA$ observation band. For the longer wavelength setting near $2113 \AA$ IUE low resolution, large aperture LWP observations show a flux of $3 \mathrm{E}-11 \mathrm{erg} / \mathrm{cm}^{2} / \mathrm{s} / \AA$ across the wavelength region of interest. These fluxes result in an exposure time of 5400 seconds at $1598 \AA$ using the $0.2 \times 0.09$ slit and at the $2113 \AA$ setting we use the $31 \mathrm{X} 0.05 \mathrm{NDA}$ and have an exposure time of 715 seconds.

Estimating overheads of 6 minutes for guide acq (and 5 for re-acq), 6 minutes each for target acq and peakup, 8 minutes for 1st MAMA exposure. Thus the set-up time is a minimum of 26 minutes and we have 55 minutes of visibility for our targets. Thus HD 184875 will take a minimum of 2 orbits, and HD 192640 will take 3 orbits.

\section{Special Requirements}

\section{None}




\section{- Coordinated Observations}

\section{None}

\section{Justify Duplications}

None

\section{Past HST Usage}

Programs in the last $4 H S T$ cycles by Lauroesch:

HST GO 12191 - PI Lauroesch "Prospecting for Rare Elements in the Interstellar Medium", data acquisition completed, data analysis completed, publication in preparation.

HST SNAP 12192 - PI Lauroesch "A SNAPSHOT Survey of Interstellar Absorption

Lines", data acquisition complete, publication detailing temporal variations toward HD 210809 in preparation. A second publication detailing limits on temporal variations toward repeated stars in planning.

Publications from previous $H S T$ programs:

Welty, D.E., Hobbs, L.M., Lauroesch, J. T., Morton, D.C., \& York, D.G. 1995, "Interstellar Lead", ApJ, 449, L149

Welty, D. E., Lauroesch, J. T., Blades, J. C., Hobbs, L. M., \& York, D. G. 1997, "Interstellar Abundances in the Magellanic Clouds. I. - GHRS Observations of the SMC Star Sk 108", ApJ, 489, 672

Welty, D. E., Lauroesch, J. T., Blades, J. C., Hobbs, L. M., \& York, D. G. 1997, "Interstellar Abundances in the Magellanic Clouds. I. - GHRS Observations of the SMC Star Sk 108", ApJ, 489, 672

Vanden Berk, D. E., Lauroesch, J. T., Stoughton, C., Szalay, A. S., Koo, D. C., Crotts, S. P. S., Blades, J. C., Melott, A. L., Boyle, B. J., Broadhurst, T. J., \& York, D. G. 1999, "Clustering Properties of Low-Redshift QSO Absorption Systems Towards the Galactic Poles", ApJS, 122, 355

Lauroesch, J. T., \& Meyer, D. M. 1999, "Observations of Small-Scale Interstellar Structure in Dense Atomic Gas", ApJ, 519, L181

Welty, D.E., Hobbs, L. M., Lauroesch, J. T., Morton, D. C., Spitzer, L., \& York, D. G. 1999, "The Diffuse Interstellar Clouds toward 23 Orionis", ApJS, 124, 465

Lauroesch, J. T., Meyer, D. M., \& Blades, J. C. 2000, "Evidence of Interstellar Na I Structure at Scales Down to 15 AU in Low-Density Gas", ApJ, 543, L43

Meyer, D. M., Lauroesch, J. T., Sofia, U. J., Draine, B. T., \& Bertoldi, F. 2001, "The Rich Ultraviolet Spectrum of Vibrationally Excited Interstellar $\mathrm{H}_{2}$ toward HD 37903", ApJ, 553, L59

Welty, D. E., Lauroesch, J. T., Blades, J. C. Hobbs, L. M., \& York, D. G. 2001, "Unusual Depletions toward the SMC Star SK 155-Differences in Dust Composition in the SMC Interstellar Medium?", ApJ, 554, L75 
Wang, Q. D., Immler, S., Walterbos, R., Lauroesch, J. T., \& Breitschwerdt, D. 2001, "Chandra Detection of a Hot Gaseous Corona around the Edge-on Galaxy NGC 4631", ApJ, 555, L99

Cartledge, S. I. B., Meyer, D. M., Lauroesch, J. T., \& Sofia, U. J. 2001, "Space Telescope Imaging Spectrograph Observations of Interstellar Oxygen and Krypton in Translucent Clouds", ApJ, 562, 394

Fox, A. J., Savage, B. D., Sembach, K. R., Fabian, D., Richter, P., Meyer, D. M., Lauroesch, J. T., \& Howk, J. Christopher 2003, "Origins of the Highly Ionized Gas along the Line of Sight Towards HD 116852", ApJ, 582, 793

Jenkins, E. B., Bowen, D. V., Tripp, T. M., Sembach, K. R., Leighly, K. M., Halpern, J. P., \& Lauroesch, J. T. 2003, "Absorption-Line Systems and Galaxies in Front of the Second Brightest Quasar, PHL 1811" AJ, 125, 2824

Knauth, D. C., Howk, J. C., Sembach, K. R., Lauroesch, J. T., \& Meyer, D. M. 2003,

"On the Origin of the High-Ionization Intermediate-Velocity Gas Toward HD 14434", ApJ, 592, 964

Cartledge, S. I. B., Meyer, D. M., \& Lauroesch, J. T. 2003, "The Homogeneity of Interstellar Krypton in the Galactic Disk", ApJ, 597, 408

Sofia, U. J., Lauroesch, J. T., Meyer, D. M., \& Cartledge, S. I. B. 2004, "Interstellar Carbon in Translucent Sightlines", ApJ, 605, 272

Cartledge, S. I. B., Lauroesch, J. T., Meyer, D. M., \& Sofia, U. J. 2004, "The Homogeneity of Interstellar Oxygen in the Galactic Disk", ApJ, 613, 1037

Kulkarni, V. P., Fall, S. M., Lauroesch, J. T., York, D. G., Khare, P., Truran, J. W., \& Welty, D. E. 2005, "Element Abundances in Low-Redshift Damped Lyman-Alpha Galaxies", ApJ, 618, 68

Cartledge, S. I. B., Lauroesch, J. T., Meyer, D. M., \& Sofia, U. J. 2006, "The Homogeneity of Interstellar Elemental Abundances in the Galactic Disk", ApJ, 641, 327

Miller, A., Lauroesch, J. T., Sofia, U. J., Cartledge, S. I. B., \& Meyer, D. M. 2007, "Interstellar Iron and Silicon Depletions in Translucent Sight Lines", ApJ, 659, 441

Cartledge, S. I. B., Lauroesch, J. T., Meyer, D. M., Sofia, U. J. \& Clayton, G. C. 2008, "Interstellar Krypton Abundances: The Detection of Kiloparsec-scale Differences in Galactic Nucleosynthetic History", ApJ, 687, 1043

Hornbeck, J. B., Grady, C. A., Perrin, M. D., Wisniewski, J. P., Tofflemire, B. M., Brown, A., Holtzman, J. A., Arraki, K., Hamaguchi, K., Woodgate, B., Petre, R., Daly, B., Grogin, N. A., Bonfield, D. G., Williger, G. M. \& Lauroesch, J. T. 2012, "PDS 144: The First Confirmed Herbig Ae - Herbig Ae Wide Binary", ApJ, 744, 54

Meyer, D. M., Lauroesch, J. T., Peek, J. E. G. \& Heiles, C. 2012, "The Remarkable High Pressure of the Local Leo Cold Cloud", ApJ, 752, 119 


\section{APPENDIX B}

\section{OBSERVATIONAL METADATA}

The tables included in this chapter have correspondence with Tables $5.1-5.11$ in Chapter 5. These tables contain the observational metadata from the Elodie archive spectra. Annotations and table order match those in Chapter 4. 
TABLE B. 1

Observational Metadata for Non-OB Measurable Targets - A

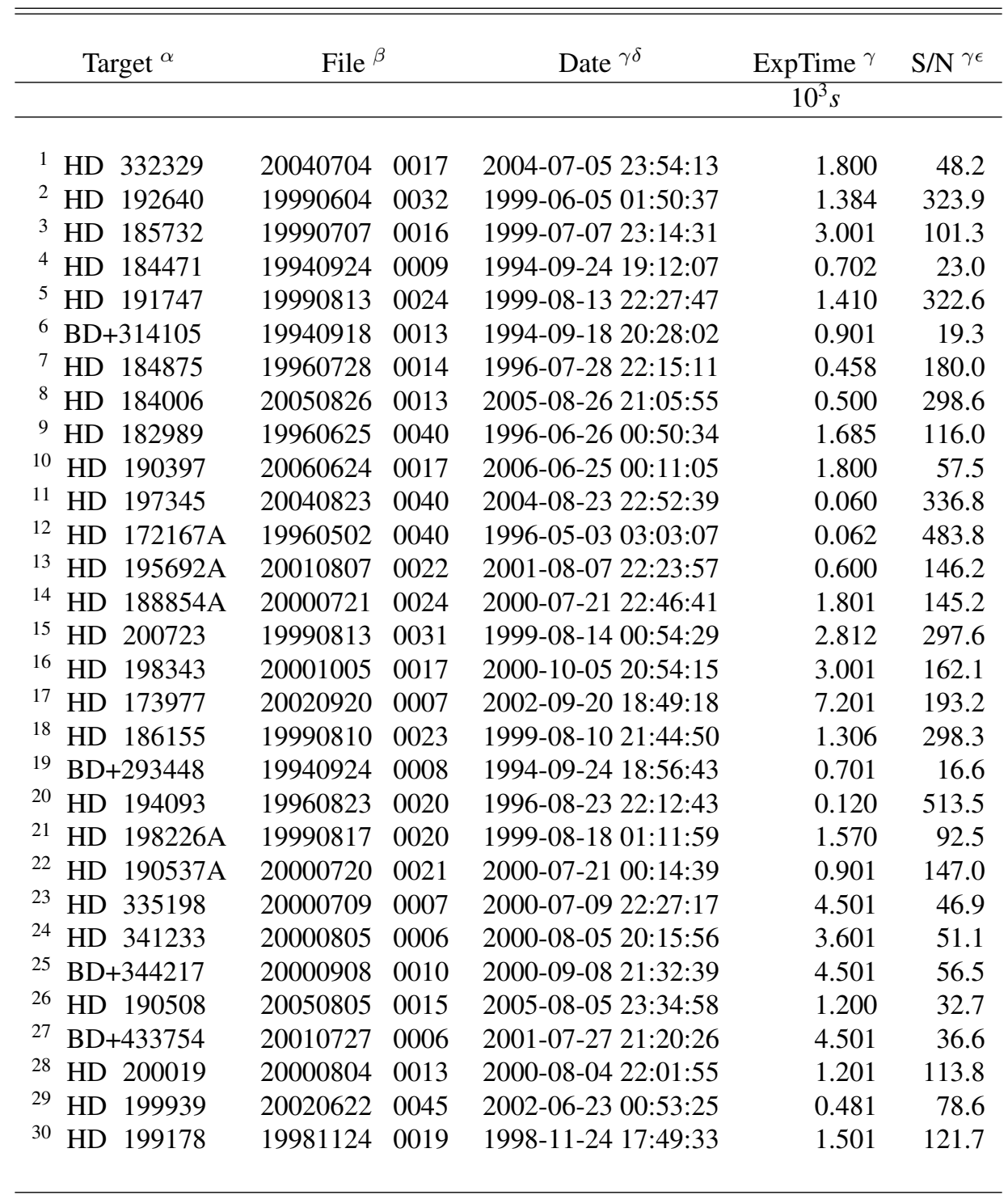

${ }^{\alpha}$ Numbers correspond to annotations in Figure 5.2 and Tables 5.1 - 5.2.

${ }^{\beta}$ Elodie file names given by "elodie:yyyymmdd/nnnn".

$\gamma$ Metadata taken directly from FITS headers.

${ }^{\delta}$ At the beginning of the exposure. Format is yyyy-mm-dd $h h: m m: s s$

${ }^{\epsilon}$ As reported by the archive. 
TABLE B.2

Observational Metadata for Non-OB Measurable Targets - B

\begin{tabular}{|c|c|c|c|c|c|c|}
\hline & Target $^{\alpha}$ & \multicolumn{2}{|c|}{ File $^{\beta}$} & Date $\gamma \delta$ & ExpTime $^{\gamma}$ & $\mathrm{S} / \mathrm{N}^{\gamma \epsilon}$ \\
\hline 31 & HD 335070 & 20010825 & 0012 & 2001-08-25 21:57:14 & 4.501 & 54.4 \\
\hline 32 & HD 193891 & 20000706 & 0007 & 2000-07-06 20:53:03 & 1.201 & 105.9 \\
\hline 33 & HD 170527 & 20031103 & 0009 & 2003-11-03 18:07:57 & 2.700 & 166.4 \\
\hline 34 & HD 198149 & 19950911 & 0017 & $1995-09-11$ 21:23:23 & 0.300 & 405.2 \\
\hline 35 & $\mathrm{BD}+483149$ & 20010731 & 0030 & 2001-08-01 00:27:20 & 1.501 & 84.0 \\
\hline 36 & HD 332698 & 20031004 & 0011 & $2003-10-04$ 20:05:47 & 5.563 & 78.1 \\
\hline 37 & HD 187372 & 20000809 & 0018 & 2000-08-09 22:12:40 & 0.601 & 182.9 \\
\hline 38 & HD 186619 & 20030703 & 0023 & $2003-07-03$ 23:23:47 & 0.300 & 76.5 \\
\hline 39 & HD 186532 & 20030703 & 0021 & 2003-07-03 23:08:41 & 0.360 & 61.1 \\
\hline 40 & HD 177808 & 20030703 & 0013 & 2003-07-03 21:39:20 & 0.360 & 79.5 \\
\hline 41 & HD 172381 & 20010802 & 0035 & 2001-08-03 00:01:44 & 0.421 & 52.2 \\
\hline 42 & HD 190964 & 20030703 & 0029 & 2003-07-04 00:17:46 & 0.300 & 54.3 \\
\hline 43 & HD 197939 & 20030703 & 0031 & 2003-07-04 00:45:06 & 0.300 & 47.2 \\
\hline
\end{tabular}

${ }^{\alpha}$ Numbers correspond to annotations in Figure 5.2 and Tables 5.1 - 5.2.

$\beta$ Elodie file names given by "elodie:yyyymmdd/nnnn".

$\gamma$ Metadata taken directly from FITS headers.

${ }^{\delta}$ At the beginning of the exposure. Format is yyyy-mm-dd $h h: m m: s s$

${ }^{\epsilon}$ As reported by the archive. 
TABLE B. 3

Observational Metadata for Non-OB No Absorption Targets - A

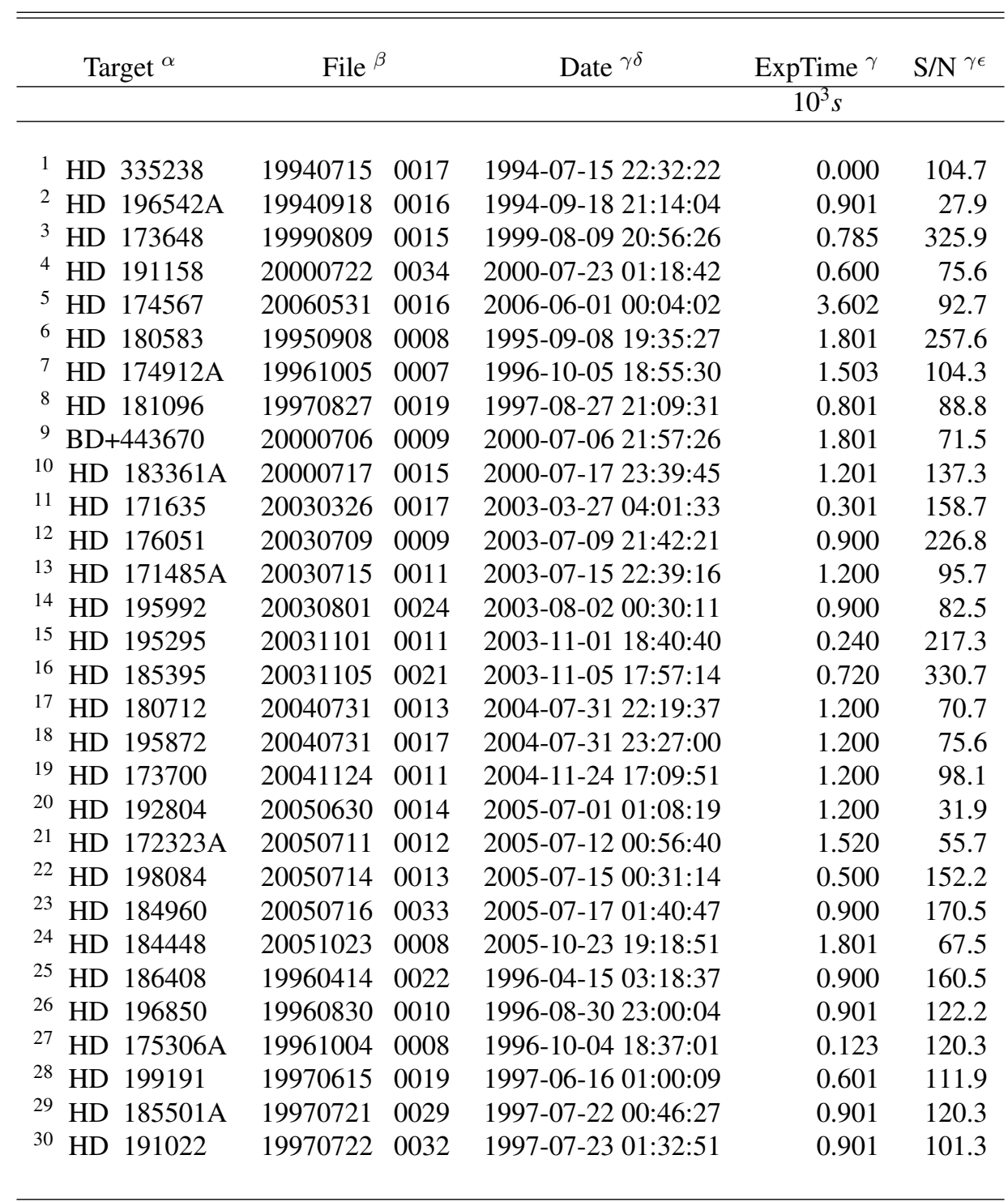

${ }^{\alpha}$ Numbers correspond to annotations in Figure 5.3 and Tables 5.3 - 5.4.

${ }^{\beta}$ Elodie file names given by "elodie:yyyymmdd/nnnn".

${ }^{\gamma}$ Metadata taken directly from FITS headers.

${ }^{\delta}$ At the beginning of the exposure. Format is yyyy-mm-dd hh:mm:ss

${ }^{\epsilon}$ As reported by the archive. 
TABLE B.4

Observational Metadata for Non-OB No Absorption Targets - B

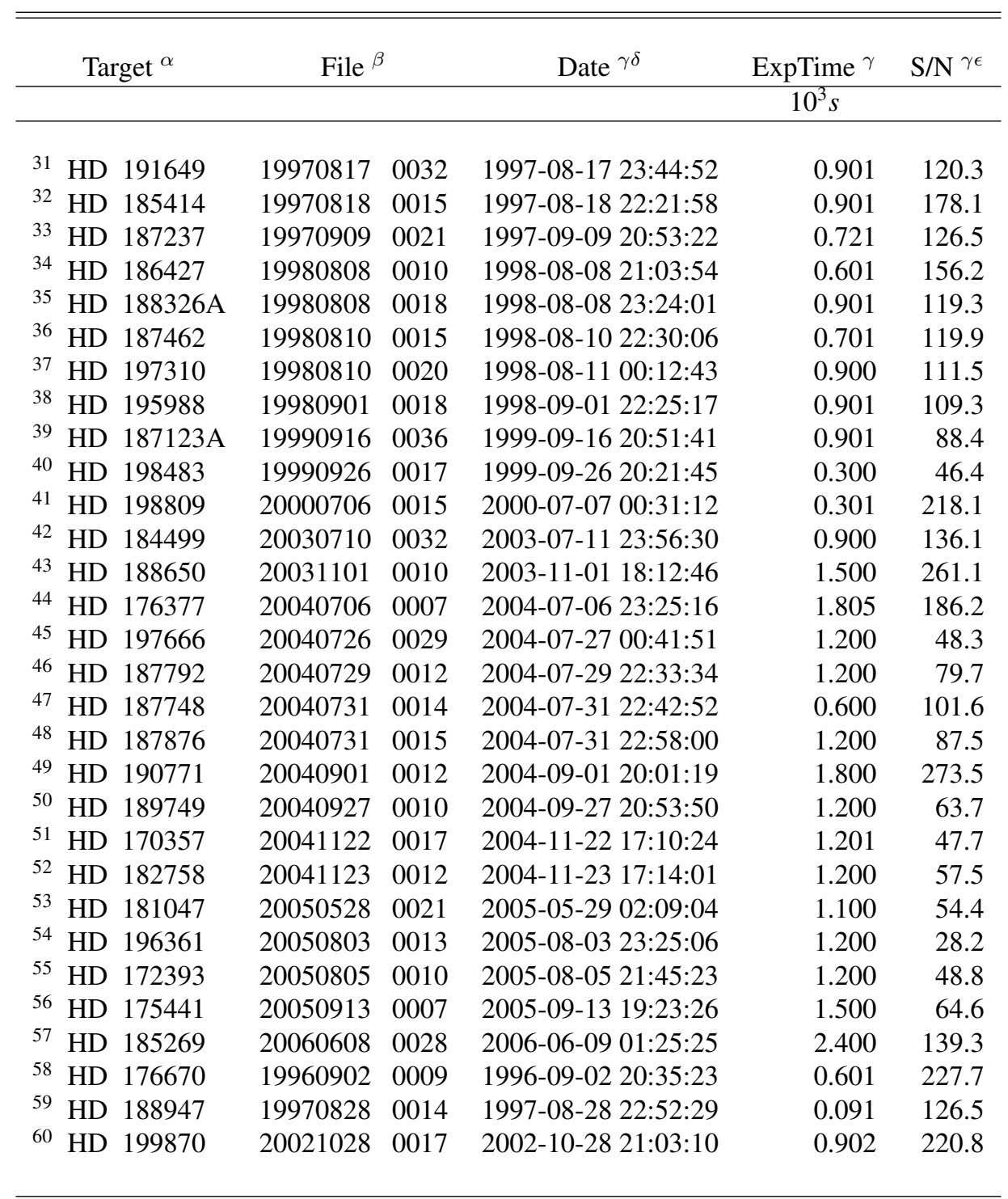

${ }^{\alpha}$ Numbers correspond to annotations in Figure 5.3 and Tables 5.3 - 5.4.

${ }^{\beta}$ Elodie file names given by "elodie:yyyymmdd/nnnn".

${ }^{\gamma}$ Metadata taken directly from FITS headers.

${ }^{\delta}$ At the beginning of the exposure. Format is yyyy-mm-dd hh:mm:ss

${ }^{\epsilon}$ As reported by the archive. 
TABLE B.5

Observational Metadata for Non-OB Inconclusive Targets - A

\begin{tabular}{|c|c|c|c|c|c|}
\hline \multirow{2}{*}{$\begin{array}{c}\text { Target } \\
\text { BD+293427 }\end{array}$} & \multicolumn{2}{|c|}{ File $^{\beta}$} & Date $^{\gamma \delta}$ & $\begin{array}{c}\text { ExpTime } \gamma \\
10^{3} s \\
\end{array}$ & $\mathrm{~S} / \mathrm{N}^{\gamma \epsilon}$ \\
\hline & 19940918 & 0009 & 1994-09-18 19:16:24 & 0.801 & 18.7 \\
\hline HD 340577 & 19940918 & 0015 & 1994-09-18 20:52:00 & 0.901 & 27.6 \\
\hline HD 200405 & 19940918 & 0023 & 1994-09-18 23:35:40 & 0.703 & 26.6 \\
\hline HD 191742 & 19940919 & 0009 & 1994-09-19 20:01:26 & 1.203 & 26.0 \\
\hline HD 192678 & 19940924 & 0012 & 1994-09-24 20:01:23 & 0.603 & 51.3 \\
\hline HD 195725 & 19990809 & 0021 & 1999-08-09 22:40:21 & 1.004 & 316.4 \\
\hline HD 177196 & 19990813 & 0019 & 1999-08-13 20:54:11 & 1.600 & 303.4 \\
\hline HD 183534 & 20060531 & 0018 & 2006-06-01 01:10:57 & 4.501 & 154.1 \\
\hline $\mathrm{BD}+353616 \mathrm{~A}$ & 19940918 & 0010 & 1994-09-18 19:36:40 & 0.602 & 17.4 \\
\hline HD 341037 & 19940924 & 0015 & 1994-09-24 20:55:23 & 0.801 & 27.7 \\
\hline HD 197572 & 19960822 & 0011 & 1996-08-22 21:00:20 & 0.000 & 324.6 \\
\hline HD 198726 & 19960823 & 0027 & 1996-08-24 00:01:34 & 2.001 & 257.3 \\
\hline BD+423935 & 19970614 & 0020 & 1997-06-15 00:59:00 & 0.601 & 19.7 \\
\hline HD 238932A & 19970617 & 0017 & 1997-06-17 23:51:12 & 0.301 & 18.9 \\
\hline BD+423607 & 19970822 & 0008 & 1997-08-22 20:57:07 & 3.602 & 59.7 \\
\hline HD 194951 & 19990706 & 0018 & 1999-07-07 02:29:49 & 0.900 & 81.7 \\
\hline HD 331319 & 19990709 & 0016 & 1999-07-10 01:06:21 & 5.401 & 98.1 \\
\hline HD 195069 & 20030625 & 0017 & 2003-06-26 00:15:07 & 7.202 & 560.0 \\
\hline HD 193370 & 20030725 & 0014 & 2003-07-26 00:30:27 & 0.660 & 241.1 \\
\hline HD 200805 & 20030729 & 0010 & 2003-07-30 00:13:00 & 4.501 & 152.7 \\
\hline HD 178593 & 20040801 & 0011 & 2004-08-01 22:23:03 & 1.506 & 103.7 \\
\hline HD 188307 & 20040802 & 0016 & 2004-08-03 23:55:22 & 1.200 & 28.6 \\
\hline HD 187253 & 20050722 & 0034 & 2005-07-23 00:41:41 & 1.200 & 31.8 \\
\hline HD 185239 & 20050913 & 0008 & 2005-09-13 19:55:05 & 1.500 & 75.3 \\
\hline HD 175225 & 19960503 & 0033 & 1996-05-04 02:09:59 & 0.601 & 216.8 \\
\hline HD 173701 & 19961023 & 0025 & 1996-10-23 18:00:10 & 0.901 & 133.0 \\
\hline HD 177153 & 19970820 & 0011 & 1997-08-20 20:55:14 & 0.901 & 132.8 \\
\hline HD 185351 & 19970823 & 0006 & 1997-08-23 20:29:39 & 0.630 & 267.0 \\
\hline BD+413931 & 19970823 & 0012 & 1997-08-23 22:30:41 & 3.601 & 68.2 \\
\hline HD 175306 & 19970827 & 0016 & 1997-08-27 20:02:16 & 0.601 & 24.4 \\
\hline
\end{tabular}

${ }^{\beta}$ Elodie file names given by "elodie:yyyymmdd/nnnn".

${ }^{\gamma}$ Metadata taken directly from FITS headers.

${ }^{\delta}$ At the beginning of the exposure. Format is yyyy-mm-dd $h h: m m: s s$

${ }^{\epsilon}$ As reported by the archive. 
TABLE B.6

Observational Metadata for Non-OB Inconclusive Targets - B

\begin{tabular}{|c|c|c|c|c|c|}
\hline Target & File ${ }^{\beta}$ & & Date $^{\gamma \delta}$ & ExpTime $\gamma$ & $\mathrm{S} / \mathrm{N}^{\gamma \epsilon}$ \\
\hline HD 182488 & 19980901 & 0009 & 1998-09-01 20:02:18 & 0.901 & 163.6 \\
\hline HD 175900 & 19990814 & 0014 & 1999-08-14 22:37:57 & 0.904 & 91.8 \\
\hline HD 193795 & 19990926 & 0015 & 1999-09-26 20:05:14 & 0.300 & 33.7 \\
\hline HD 200391 & 20000707 & 0007 & 2000-07-07 21:35:17 & 1.201 & 95.2 \\
\hline HD 181655 & 20000731 & 0015 & 2000-07-31 23:06:50 & 0.751 & 183.9 \\
\hline BD+364301 & 20000804 & 0016 & 2000-08-04 23:21:21 & 3.601 & 74.8 \\
\hline HD 179484A & 20000813 & 0020 & $2000-08-13$ 22:27:28 & 1.801 & 73.8 \\
\hline HD 190360A & 20000824 & 0023 & 2000-08-24 23:03:32 & 0.721 & 206.8 \\
\hline HD 178911A & 20010402 & 0032 & 2001-04-03 02:59:15 & 1.201 & 107.3 \\
\hline HD 199598 & 20010721 & 0021 & 2001-07-22 00:46:46 & 0.901 & 151.9 \\
\hline HD 173605 & 20010728 & 0005 & 2001-07-28 20:09:56 & 1.501 & 127.5 \\
\hline HD 190228 & 20010811 & 0015 & 2001-08-11 22:12:07 & 0.901 & 125.9 \\
\hline HD 188326 & 20010812 & 0017 & 2001-08-12 22:25:24 & 1.501 & 112.7 \\
\hline HD 187123 & 20010814 & 0018 & 2001-08-14 22:52:25 & 1.001 & 96.4 \\
\hline HD 182736 & 20011010 & 0022 & 2001-10-10 21:42:19 & 0.901 & 116.6 \\
\hline HD 171242 & 20011011 & 0012 & 2001-10-11 19:32:37 & 0.901 & 75.5 \\
\hline HD 185657 & 20021028 & 0015 & 2002-10-28 20:24:06 & 1.343 & 200.9 \\
\hline HD 171007 & 20030521 & 0022 & 2003-05-22 00:49:22 & 1.801 & 42.3 \\
\hline HD 193216A & 20030715 & 0013 & 2003-07-15 23:21:17 & 0.900 & 50.1 \\
\hline HD 178911B & 20030717 & 0020 & 2003-07-18 00:47:04 & 0.900 & 117.0 \\
\hline HD 200102 & 20030725 & 0019 & 2003-07-26 02:02:02 & 3.001 & 258.1 \\
\hline HD 190403 & 20030727 & 0011 & 2003-07-27 23:26:14 & 3.601 & 156.8 \\
\hline HD 174104 & 20030729 & 0007 & 2003-07-29 20:08:57 & 5.001 & 122.4 \\
\hline HD 191010 & 20030729 & 0009 & 2003-07-29 22:53:58 & 4.201 & 149.7 \\
\hline HD 180161 & 20040710 & 0010 & 2004-07-11 00:43:52 & 2.401 & 129.8 \\
\hline HD 180683 & 20040726 & 0024 & 2004-07-26 22:57:15 & 1.200 & 50.4 \\
\hline HD 197037 & 20040726 & 0027 & 2004-07-27 23:53:04 & 1.200 & 75.0 \\
\hline HD 180502 & 20040728 & 0012 & 2004-07-28 22:20:08 & 1.200 & 80.1 \\
\hline HD 197488 & 20040728 & 0016 & 2004-07-29 23:58:53 & 1.200 & 79.0 \\
\hline HD 175425 & 20040926 & 0012 & 2004-09-26 19:17:56 & 1.200 & 57.1 \\
\hline
\end{tabular}

${ }^{\beta}$ Elodie file names given by "elodie:yyyymmdd/nnnn".

${ }^{\gamma}$ Metadata taken directly from FITS headers.

${ }^{\delta}$ At the beginning of the exposure. Format is yyyy-mm-dd $h h: m m: s s$

${ }^{\epsilon}$ As reported by the archive. 
TABLE B.7

Observational Metadata for Non-OB Inconclusive Targets - C

\begin{tabular}{|c|c|c|c|c|c|}
\hline Target & File $^{\beta}$ & & Date $^{\gamma \delta}$ & ExpTime $^{\gamma}$ & $\mathrm{S} / \mathrm{N} \gamma \epsilon$ \\
\hline HD 193215 & 20041001 & 0010 & 2004-10-01 19:11:51 & 1.200 & 57.1 \\
\hline HD 172557 & 20041002 & 0009 & 2004-10-02 18:44:12 & 1.200 & 60.3 \\
\hline HD 184601 & 20041002 & 0011 & 2004-10-02 19:30:53 & 1.200 & 69.1 \\
\hline HD 199100 & 20041002 & 0013 & 2004-10-02 20:16:51 & 1.200 & 91.8 \\
\hline HD 195987 & 20041009 & 0008 & 2004-10-09 17:53:28 & 1.802 & 126.3 \\
\hline HD 187548 & 20050529 & 0016 & 2005-05-30 00:28:37 & 1.500 & 43.9 \\
\hline HD 177780 & 20050530 & 0015 & 2005-05-31 01:51:44 & 1.500 & 22.8 \\
\hline HD 190605 & 20050616 & 0029 & 2005-06-17 02:09:52 & 1.200 & 82.6 \\
\hline HD 178911 & 20050711 & 0011 & 2005-07-12 00:25:49 & 1.017 & 110.0 \\
\hline HD 178450 & 20050714 & 0009 & 2005-07-14 22:56:23 & 2.108 & 88.6 \\
\hline HD 173024 & 20050723 & 0015 & 2005-07-23 21:41:57 & 1.200 & 35.2 \\
\hline HD 173289 & 20050725 & 0022 & 2005-07-26 00:05:31 & 1.200 & 38.3 \\
\hline HD 197140 & 20050805 & 0016 & 2005-08-06 00:02:23 & 1.200 & 44.3 \\
\hline HD 197207 & 20050912 & 0014 & 2005-09-12 21:36:10 & 1.500 & 58.4 \\
\hline HD 189087 & 19961023 & 0026 & 1996-10-23 18:19:44 & 0.801 & 103.7 \\
\hline HD 234677 & 19970521 & 0013 & 1997-05-22 00:54:31 & 3.601 & 134.7 \\
\hline HD 197989 & 19970823 & 0010 & 1997-08-23 21:16:55 & 0.021 & 204.7 \\
\hline HD 183255 & 19970924 & 0011 & $1997-09-24$ 19:45:41 & 5.401 & 215.5 \\
\hline HD 191026 & 19971108 & 0009 & 1997-11-08 18:31:03 & 1.200 & 226.7 \\
\hline HD 338867 & 19980318 & 0029 & 1998-03-19 03:24:27 & 0.602 & 43.7 \\
\hline HD 199956 & 20000708 & 0006 & 2000-07-08 22:08:06 & 2.401 & 140.0 \\
\hline HD 334514 & 20000709 & 0006 & 2000-07-09 21:06:31 & 4.501 & 36.1 \\
\hline HD 199546 & 20010730 & 0018 & 2001-07-30 20:39:07 & 1.201 & 91.2 \\
\hline HD 189806 & 20010731 & 0024 & 2001-07-31 20:28:22 & 1.201 & 76.8 \\
\hline HD 181209 & 20010731 & 0025 & 2001-07-31 20:55:43 & 1.202 & 68.2 \\
\hline HD 188056 & 20010809 & 0028 & 2001-08-09 22:28:08 & 0.603 & 95.3 \\
\hline $\mathrm{BD}+333930$ & 20010825 & 0011 & 2001-08-25 21:11:28 & 2.401 & 124.5 \\
\hline $\mathrm{BD}+334140$ & 20010826 & 0009 & 2001-08-26 21:53:42 & 3.601 & 58.8 \\
\hline HD 192787 & 20021022 & 0008 & 2002-10-22 18:07:01 & 1.641 & 261.8 \\
\hline HD 196134 & 20021027 & 0014 & 2002-10-27 20:49:15 & 2.401 & 209.3 \\
\hline
\end{tabular}

${ }^{\beta}$ Elodie file names given by “elodie:yyyymmdd/nnnn".

$\gamma$ Metadata taken directly from FITS headers.

${ }^{\delta}$ At the beginning of the exposure. Format is yyyy-mm-dd $h h: m m: s s$

${ }^{\epsilon}$ As reported by the archive. 
TABLE B.8

Observational Metadata for Non-OB Inconclusive Targets - D

\begin{tabular}{|c|c|c|c|c|c|}
\hline \multirow{2}{*}{$\begin{array}{c}\text { Target } \\
\text { HD } 176408\end{array}$} & \multicolumn{2}{|c|}{ File ${ }^{\beta}$} & Date $^{\gamma \delta}$ & $\frac{\text { ExpTime }^{\gamma}}{10^{3} \mathrm{~s}}$ & $\mathrm{~S} / \mathrm{N}^{\gamma \epsilon}$ \\
\hline & 20021028 & 0013 & $2002-10-28$ 19:57:56 & 0.900 & 274.0 \\
\hline HD 198550 & 20030711 & 0031 & $2003-07-1200: 47: 33$ & 0.900 & 59.4 \\
\hline HD 192910 & 20030725 & 0013 & 2003-07-26 00:14:37 & 0.360 & 152.3 \\
\hline HD 193469 & 20030726 & 0017 & 2003-07-27 02:15:11 & 2.101 & 188.3 \\
\hline HD 198794 & 20030727 & 0012 & $2003-07-2800: 32: 39$ & 3.600 & 114.6 \\
\hline HD 200560 & 20031102 & 0010 & 2003-11-02 19:16:11 & 3.602 & 139.7 \\
\hline HD 190470 & 20040712 & 0012 & 2004-07-13 01:33:42 & 3.300 & 70.5 \\
\hline BD+413306 & 20040819 & 0017 & 2004-08-19 21:05:36 & 3.600 & 85.2 \\
\hline $\mathrm{BD}+522815$ & 20040902 & 0009 & 2004-09-02 20:41:52 & 5.401 & 95.9 \\
\hline HD 332518 & 20040907 & 0008 & 2004-09-07 20:43:30 & 6.301 & 84.6 \\
\hline HD 331093 & 20040926 & 0014 & 2004-09-26 20:05:21 & 1.200 & 36.8 \\
\hline HD 191806 & 20040928 & 0008 & 2004-09-28 20:18:13 & 1.200 & 66.8 \\
\hline HD 171607 & 20041002 & 0008 & 2004-10-02 18:21:04 & 1.200 & 70.1 \\
\hline HD 179094 & 20050625 & 0022 & 2005-06-26 02:16:58 & 0.600 & 118.5 \\
\hline HD 198425 & 20050703 & 0019 & 2005-07-04 01:25:28 & 1.200 & 51.0 \\
\hline HD 188753 & 20051025 & 0006 & $2005-10-25$ 18:54:47 & 1.801 & 111.1 \\
\hline $\mathrm{BD}+400883$ & 19940928 & 0015 & 1994-09-28 19:51:46 & 3.601 & 35.1 \\
\hline HD 173739 & 19970524 & 0023 & 1997-05-23 22:20:39 & 2.701 & 109.2 \\
\hline GJ 0747 & 19970812 & 0018 & 1997-08-12 21:10:39 & 2.402 & 29.2 \\
\hline GJ 0809 & 19980723 & 0020 & 1998-07-24 00:35:53 & 1.201 & 75.4 \\
\hline HD 186686 & 19980805 & 0018 & 1998-08-05 23:18:11 & 1.801 & 204.8 \\
\hline GJ 0725 & 19990702 & 0012 & 1999-07-02 23:44:29 & 1.201 & 63.0 \\
\hline BD+394208 & 19990821 & 0014 & 1999-08-21 21:24:26 & 5.952 & 164.2 \\
\hline $\mathrm{BD}+364025$ & 19990823 & 0014 & 1999-08-23 20:26:11 & 7.201 & 152.9 \\
\hline HD 182190 & 20000809 & 0017 & 2000-08-09 21:57:55 & 0.601 & 190.7 \\
\hline HD 199871 & 20000809 & 0021 & 2000-08-09 22:55:12 & 0.900 & 131.3 \\
\hline HD 186776 & 20000810 & 0014 & 2000-08-10 22:09:23 & 0.601 & 141.0 \\
\hline HD 175865 & 20010809 & 0025 & 2001-08-09 21:36:41 & 0.601 & 255.7 \\
\hline HD 175588 & 20010810 & 0013 & 2001-08-10 21:21:48 & 0.181 & 93.1 \\
\hline HD 184786 & 20010812 & 0014 & 2001-08-12 21:46:43 & 0.361 & 121.1 \\
\hline
\end{tabular}

${ }^{\beta}$ Elodie file names given by “elodie:yyyymmdd/nnnn".

${ }^{\gamma}$ Metadata taken directly from FITS headers.

${ }^{\delta}$ At the beginning of the exposure. Format is yyyy-mm-dd hh:mm:ss

${ }^{\epsilon}$ As reported by the archive. 
TABLE B.9

Observational Metadata for Non-OB Inconclusive Targets - E

\begin{tabular}{|c|c|c|c|c|c|}
\hline Target & File ${ }^{\beta}$ & & Date $^{\gamma \delta}$ & ExpTime ${ }^{\gamma}$ & $\mathrm{S} / \mathrm{N} \gamma \epsilon$ \\
\hline GJ 0815 & 20010902 & 0018 & 2001-09-02 21:36:39 & 1.201 & 29.7 \\
\hline GJ 0766 & 20011030 & 0010 & 2001-10-30 19:09:25 & 3.601 & 12.7 \\
\hline HD 189063 & 20020618 & 0027 & 2002-06-19 01:53:06 & 0.482 & 79.8 \\
\hline HD 178003 & 20030703 & 0014 & 2003-07-03 21:49:54 & 0.360 & 49.3 \\
\hline HD 180450 & 20030703 & 0017 & $2003-07-03$ 22:23:43 & 0.360 & 45.2 \\
\hline HD 179869 & 20030703 & 0018 & 2003-07-03 22:34:21 & 0.401 & 52.3 \\
\hline HD 187849 & 20030703 & 0024 & $2003-07-03$ 23:32:47 & 0.300 & 98.5 \\
\hline HD 190544 & 20030703 & 0026 & 2003-07-03 23:52:15 & 0.300 & 88.8 \\
\hline HD 199305 & 20040818 & 0013 & 2004-08-18 22:22:13 & 3.601 & 81.3 \\
\hline HD 173740 & 20040822 & 0011 & 2004-08-22 21:34:21 & 7.201 & 104.9 \\
\hline HD 331161 & 19940925 & 0014 & 1994-09-25 20:14:54 & 3.601 & 17.6 \\
\hline HD 190163 & 20000809 & 0020 & 2000-08-09 22:34:45 & 0.900 & 67.4 \\
\hline HD 182917 & 20030703 & 0019 & $2003-07-0322: 44: 36$ & 0.500 & 50.6 \\
\hline GJ 0802 & 20050922 & 0012 & 2005-09-22 21:11:31 & 3.601 & 4.9 \\
\hline
\end{tabular}

${ }^{\beta}$ Elodie file names given by "elodie:yyyymmdd/nnnn".

$\gamma$ Metadata taken directly from FITS headers.

${ }^{\delta}$ At the beginning of the exposure. Format is yyyy-mm-dd hh:mm:ss

${ }^{\epsilon}$ As reported by the archive. 
TABLE B. 10

Observational Metadata for OB Targets - A

\begin{tabular}{|c|c|c|c|c|c|}
\hline Target & \multicolumn{2}{|c|}{ File $^{\beta}$} & Date $^{\gamma \delta}$ & $\frac{\text { ExpTime }^{\gamma}}{10^{3} s}$ & $\mathrm{~S} / \mathrm{N}^{\gamma \epsilon}$ \\
\hline HD 188209 & 19970917 & 0012 & 1997-09-17 21:36:34 & 1.803 & 417.5 \\
\hline HD 198820 & 20041110 & 0019 & 2004-11-10 19:10:20 & 3.601 & 161.1 \\
\hline HD 194335 & 19970921 & 0027 & 1997-09-21 23:04:10 & 1.201 & 243.6 \\
\hline HD 200120 & 20030808 & 0014 & 2003-08-08 22:09:33 & 1.200 & 376.4 \\
\hline HD 182255 & 20031106 & 0018 & 2003-11-06 17:37:00 & 3.601 & 512.2 \\
\hline HD 338529 & 19970822 & 0009 & 1997-08-22 22:04:28 & 3.601 & 83.9 \\
\hline HD 183056 & 20000519 & 0019 & 2000-05-20 01:15:20 & 1.801 & 268.1 \\
\hline HD 174638 & 20010906 & 0025 & 2001-09-06 21:22:35 & 0.241 & 249.7 \\
\hline HD 176437 & 19960425 & 0031 & 1996-04-26 02:27:27 & 0.301 & 64.5 \\
\hline HD 180163 & 19981128 & 0015 & 1998-11-28 18:33:38 & 0.090 & 114.3 \\
\hline HD 192685 & 20030819 & 0020 & 2003-08-19 22:17:13 & 0.901 & 371.6 \\
\hline HD 198183 & 20040823 & 0041 & 2004-08-23 23:05:39 & 0.600 & 85.9 \\
\hline BD+404124 & 19970707 & 0008 & 1997-07-07 22:02:19 & 5.401 & 62.9 \\
\hline HD 194279 & 19970919 & 0021 & 1997-09-19 22:39:34 & 3.601 & 296.8 \\
\hline HD 172324 & 20001008 & 0011 & 2000-10-08 19:51:41 & 6.001 & 152.9 \\
\hline BD+413731 & 19970708 & 0011 & $1997-07-08$ 22:27:27 & 3.601 & 93.6 \\
\hline HD 193322A & 20050817 & 0007 & 2005-08-17 19:33:11 & 1.200 & 54.6 \\
\hline HD 193322 & 20040829 & 0016 & 2004-08-29 23:18:09 & 1.200 & 189.4 \\
\hline HD 195592 & 19970918 & 0029 & 1997-09-18 20:52:24 & 3.601 & 322.8 \\
\hline HD 190864 & 20010812 & 0018 & 2001-08-12 22:56:35 & 2.402 & 144.2 \\
\hline HD 191423 & 20040829 & 0013 & 2004-08-29 19:39:37 & 5.401 & 150.5 \\
\hline HD 189957 & 20040827 & 0014 & 2004-08-27 19:38:55 & 2.701 & 137.0 \\
\hline HD 192639 & 20010811 & 0018 & 2001-08-11 22:49:26 & 0.721 & 129.6 \\
\hline HD 191978 & 20040829 & 0014 & 2004-08-29 21:14:58 & 5.401 & 125.7 \\
\hline HD 190429 & 20040827 & 0015 & 2004-08-27 20:39:05 & 1.500 & 174.4 \\
\hline HD 199579 & 19970921 & 0016 & 1997-09-21 20:49:31 & 1.508 & 248.1 \\
\hline HD 193514 & 20040828 & 0008 & 2004-08-29 00:04:36 & 2.401 & 129.4 \\
\hline HD 190429A & 20051106 & 0008 & 2005-11-06 18:38:01 & 3.601 & 154.9 \\
\hline HD 186980 & 20010811 & 0014 & 2001-08-11 21:43:19 & 1.501 & 136.0 \\
\hline HD 193443 & 20040828 & 0007 & 2004-08-28 23:08:18 & 1.800 & 157.5 \\
\hline
\end{tabular}

${ }^{\beta}$ Elodie file names given by "elodie:yyyymmdd/nnnn".

${ }^{\gamma}$ Metadata taken directly from FITS headers.

${ }^{\delta}$ At the beginning of the exposure. Format is yyyy-mm-dd hh:mm:ss

${ }^{\epsilon}$ As reported by the archive. 
TABLE B.11

Observational Metadata for OB Targets - B

\begin{tabular}{cccccc}
\hline \hline \multicolumn{1}{c}{ Target } & \multicolumn{2}{c}{ File $^{\beta}$} & Date $^{\gamma \delta}$ & ExpTime $^{\gamma}$ & $\mathrm{S} / \mathrm{N}^{\gamma \epsilon}$ \\
\hline & & & & $10^{3} s$ & \\
\hline HD 191612 & 20040828 & 0003 & $2004-08-28 ~ 19: 16: 22$ & 3.601 & 152.4 \\
HD 192281 & 20040828 & 0005 & $2004-08-2821: 33: 21$ & 2.701 & 153.0 \\
HD 191201 & 20040827 & 0017 & $2004-08-2721: 43: 10$ & 1.600 & 142.4 \\
HD 193237 & 19980614 & 0031 & $1998-06-1501: 02: 40$ & 1.800 & 250.5 \\
HD 198478 & 20040824 & 0038 & $2004-08-2421: 44: 20$ & 0.900 & 158.0 \\
HD 228712 & 19970917 & 0011 & $1997-09-1719: 28: 57$ & 7.201 & 226.3 \\
HD 194839 & 19970919 & 0008 & $1997-09-1918: 52: 58$ & 5.401 & 279.9 \\
& & & & & \\
\hline
\end{tabular}

${ }^{\beta}$ Elodie file names given by "elodie:yyyymmdd/nnnn".

$\gamma$ Metadata taken directly from FITS headers.

${ }^{\delta}$ At the beginning of the exposure. Format is yyyy-mm-dd hh: mm :ss

${ }^{\epsilon}$ As reported by the archive. 


\section{APPENDIX C}

\section{SOURCE CODE}

The following appendix contains most of the relevant source code for the SLiPy library. Each module is included in its own section for which the name reflects the file structure to that module. Some modules are not included for practical reasons. The code is self documenting using Python's docstring functionality (accounting for some of its length). The software can be downloaded from its GitHub repository at http://github.com/glentner/slipy or from its website at http://glentner.github.io/slipy. For interactive, human readable documentation visit the website. The code can be downloaded via several formats including zip and tar.gz or can be cloned directly to your desktop.

The LICENSE and README file have been omitted. This content can be accessed online. Also, the AtomicData module has been omitted intentionally. It would occupy up to a hundred or more pages of raw data in the form of a Python list and serves

no benefit to be included here. Neither are any of the files from the SLiPy.Data.Archives included. 
C.1 .. _ init

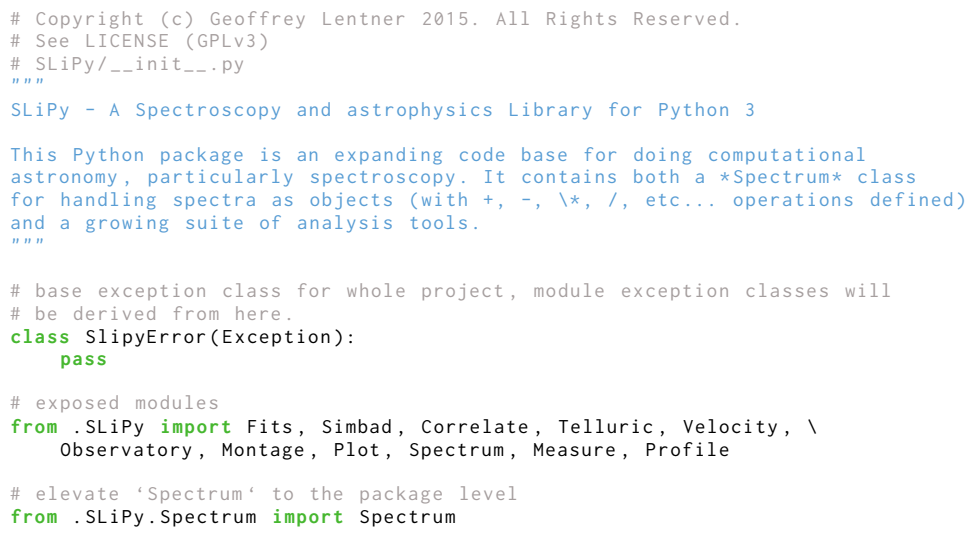




\section{C.2 .. Algorithms . Functions}

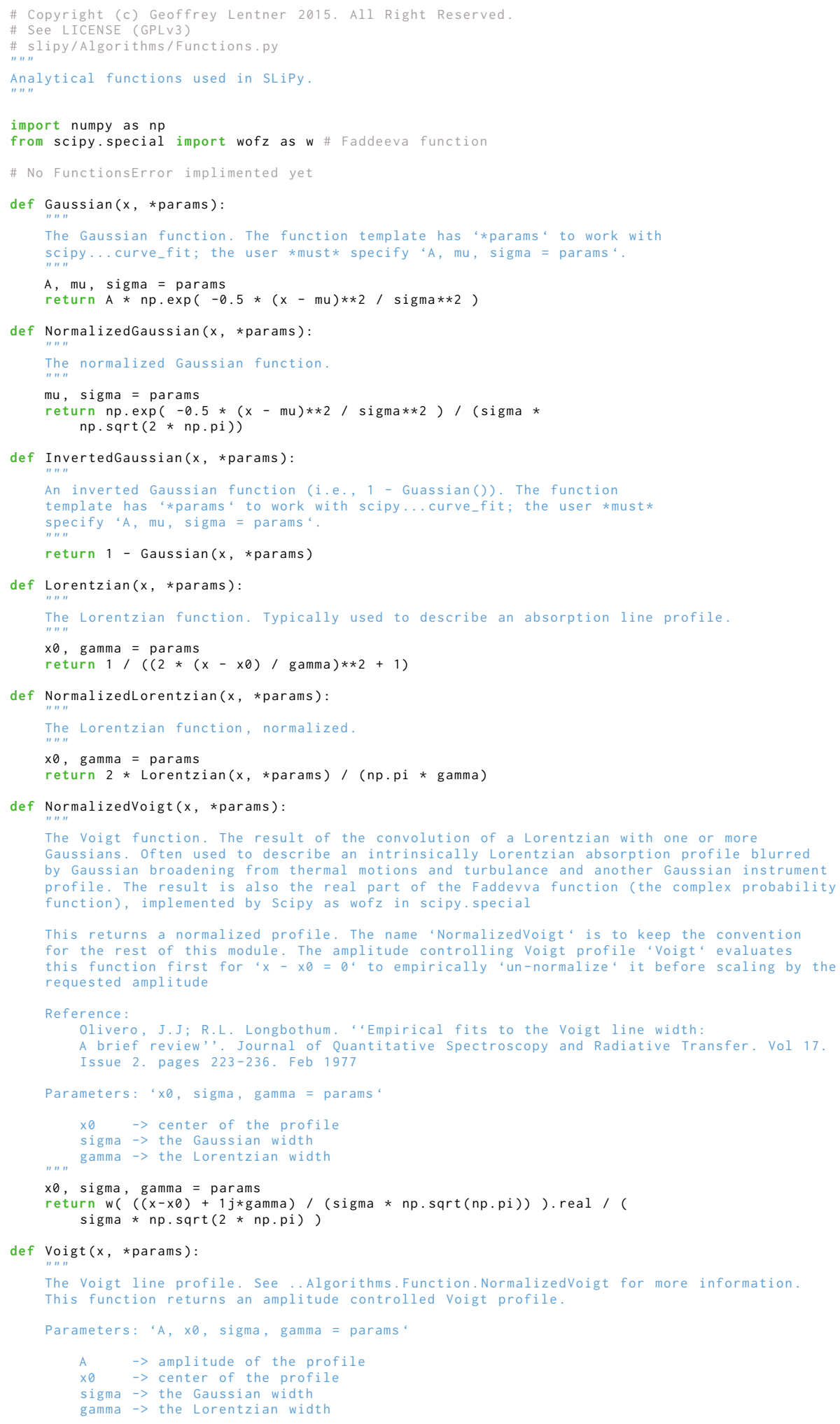




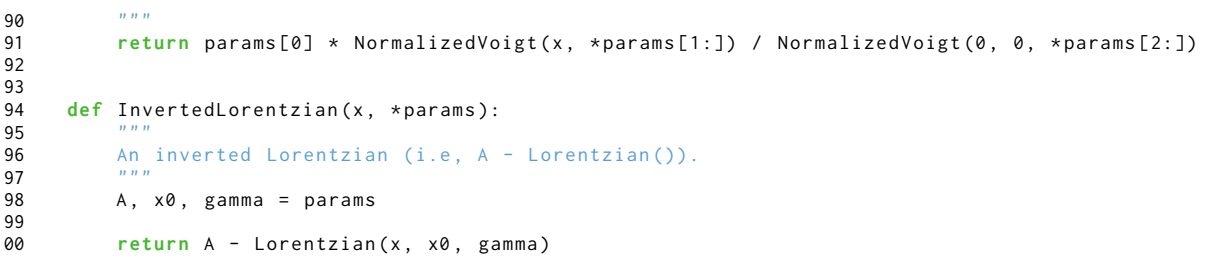




\section{C.3 .. Algorithms . KernelFit}

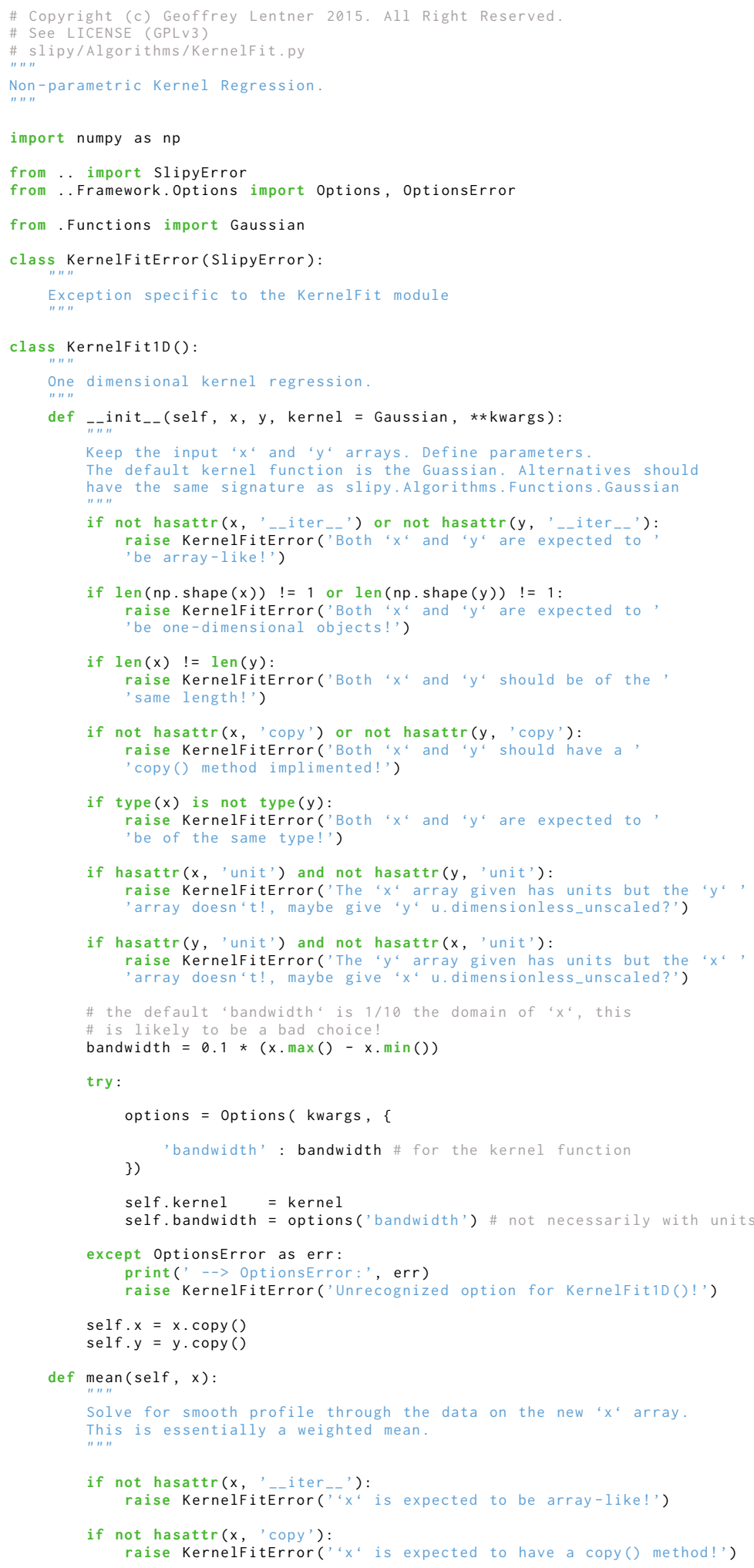


if hasattr(x, 'unit') and not hasattr(self.x, 'unit'):

raise Kernelfiterror ('The provided array has units but the '

'original domain does not!')

if hasattr(self.x, 'unit') and not hasattr(x, 'unit'):

raise Kernelfiterror('The provided array does not have units '

'but the original domain did!')

\# copy " $x$ " such that the return type is the same as the input type

$y=x \cdot \operatorname{copy}()$

\# fix units though

if hasattr(x, 'unit') :

$y=y$.value $*$ self.y.unit

for a, point in enumerate $(x)$ :

weights = Gaussian(point, 1 , self.x, self.bandwidth)

\# weights should be dimensionless

if hasattr(x, 'unit'):

weights = weights. decompose ()

$\operatorname{args}=n p$. where $(n p$. isfinite $(\operatorname{self} . y))$

$y[a]=n p . s u m($ weights[args] $*$ self.y[args]) / np.sum(weights[args])

return y 


\section{C.4 .. Data . Atomic}

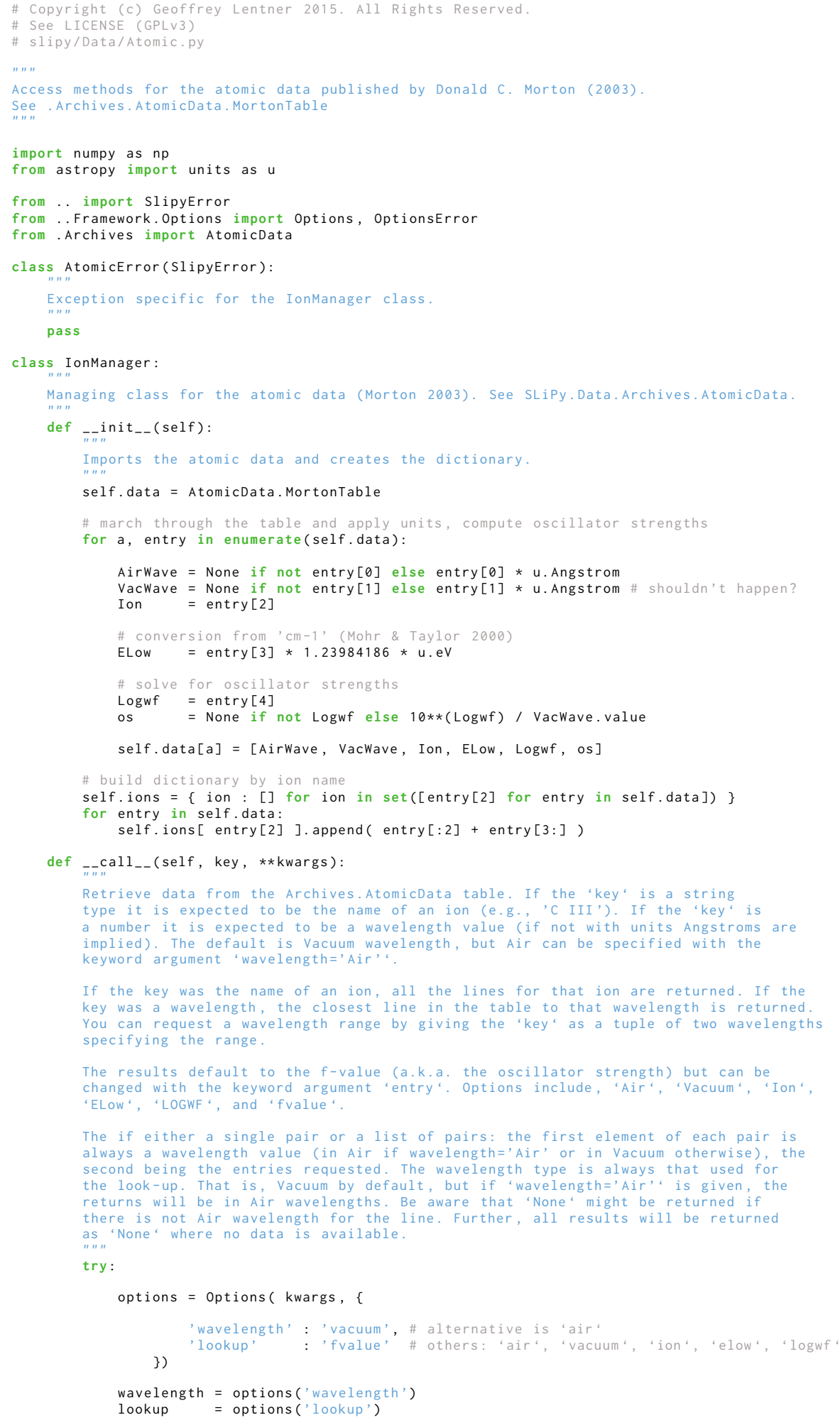




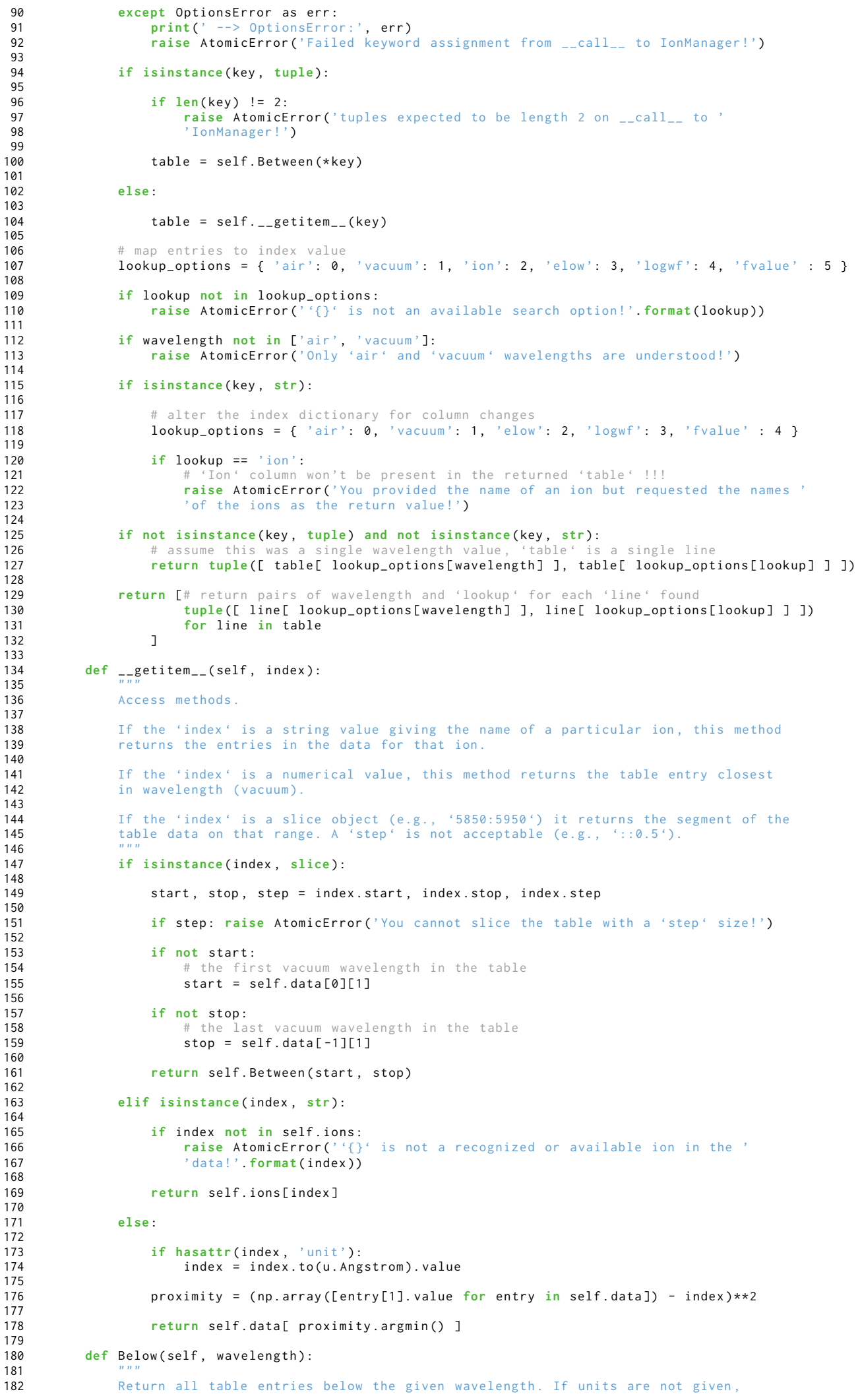




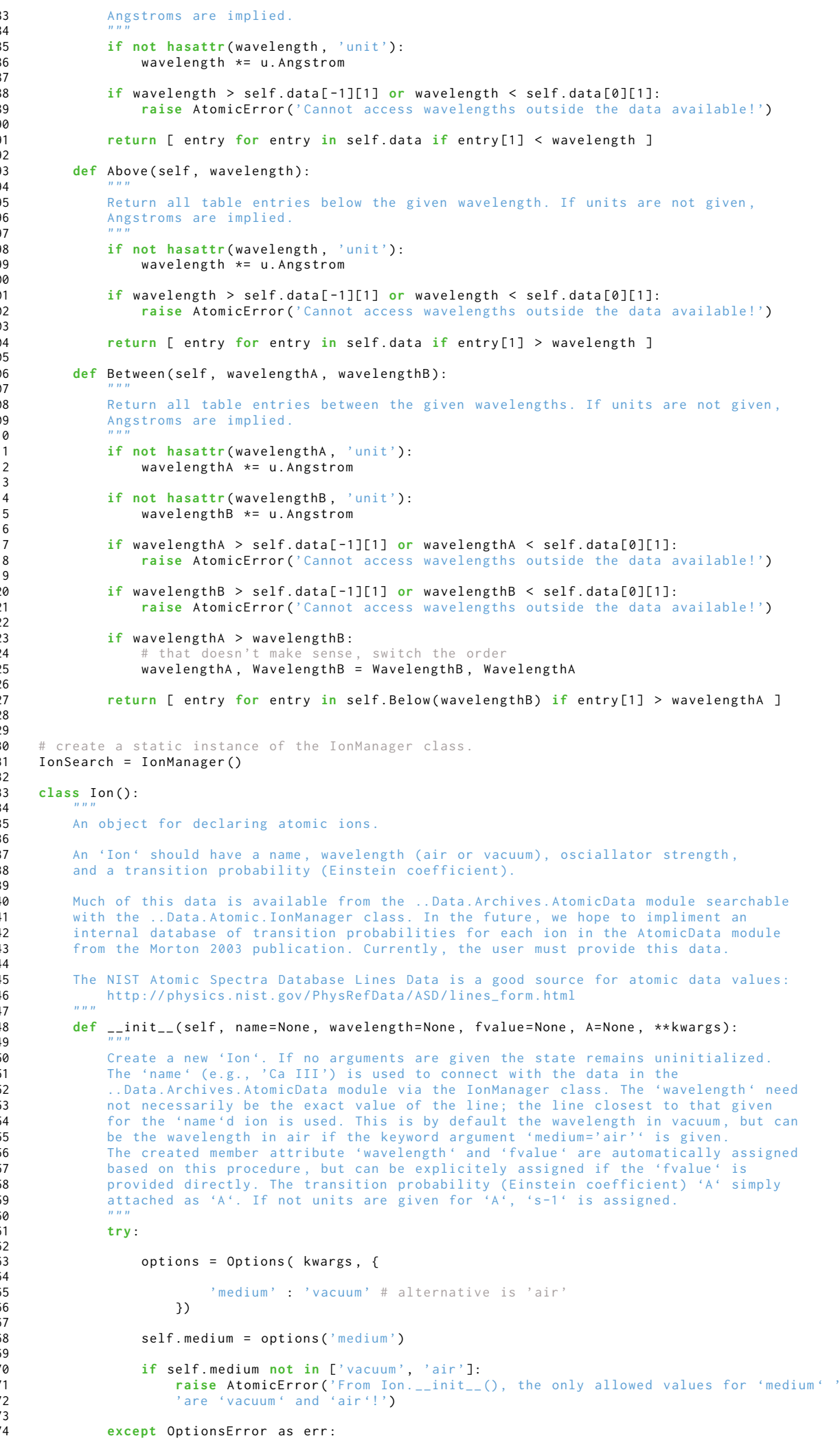




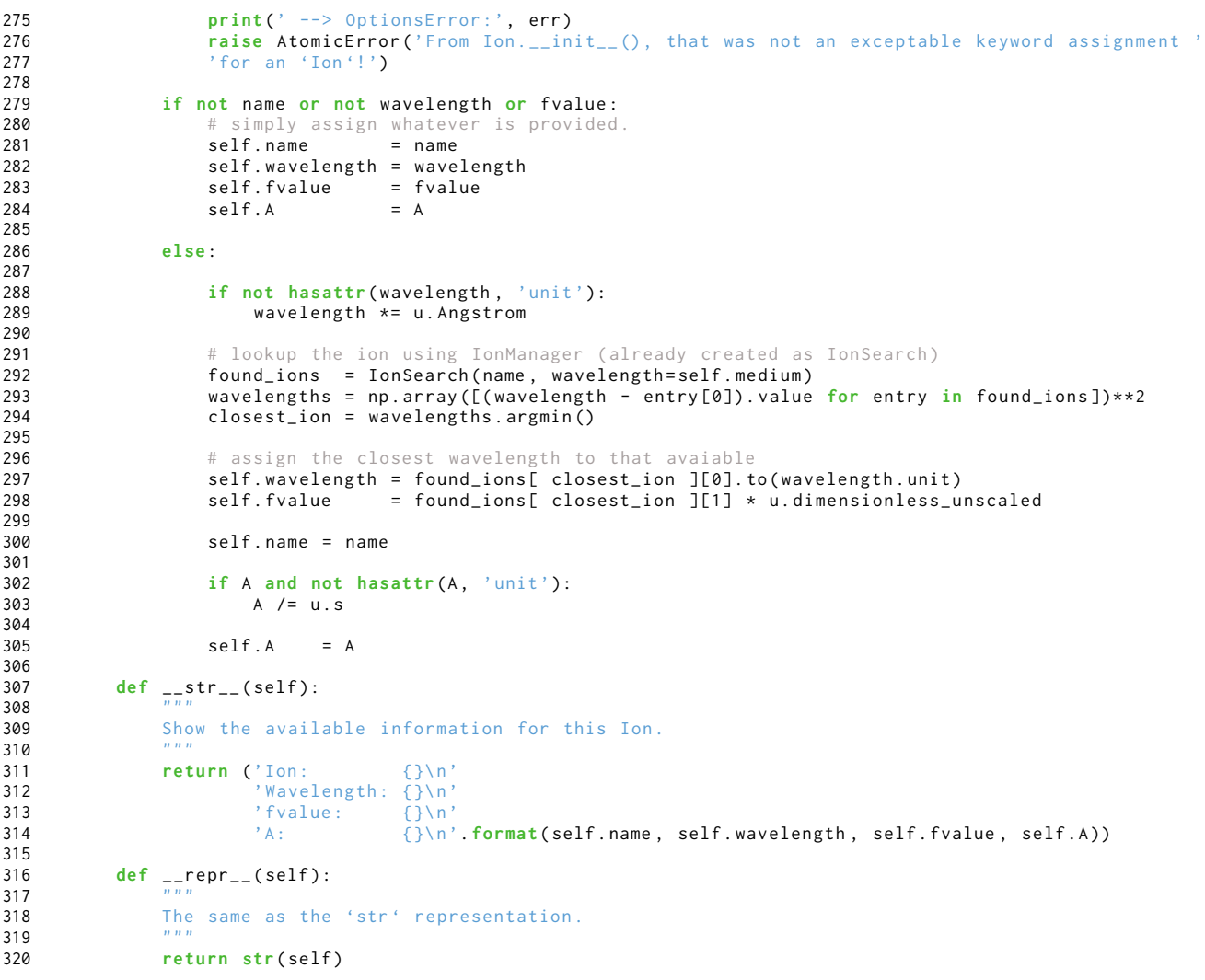




\section{C.5 .. Data . Elodie}

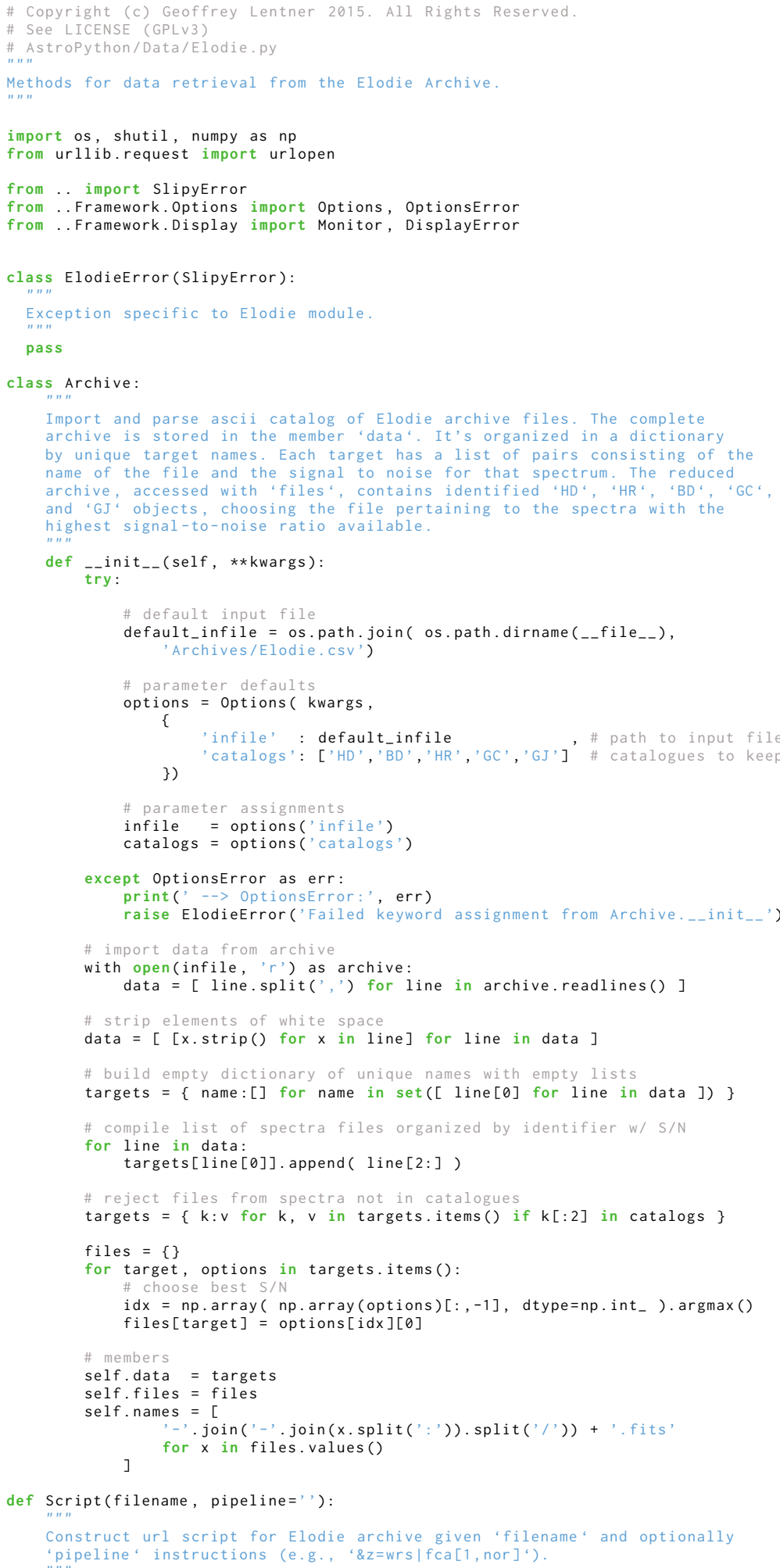




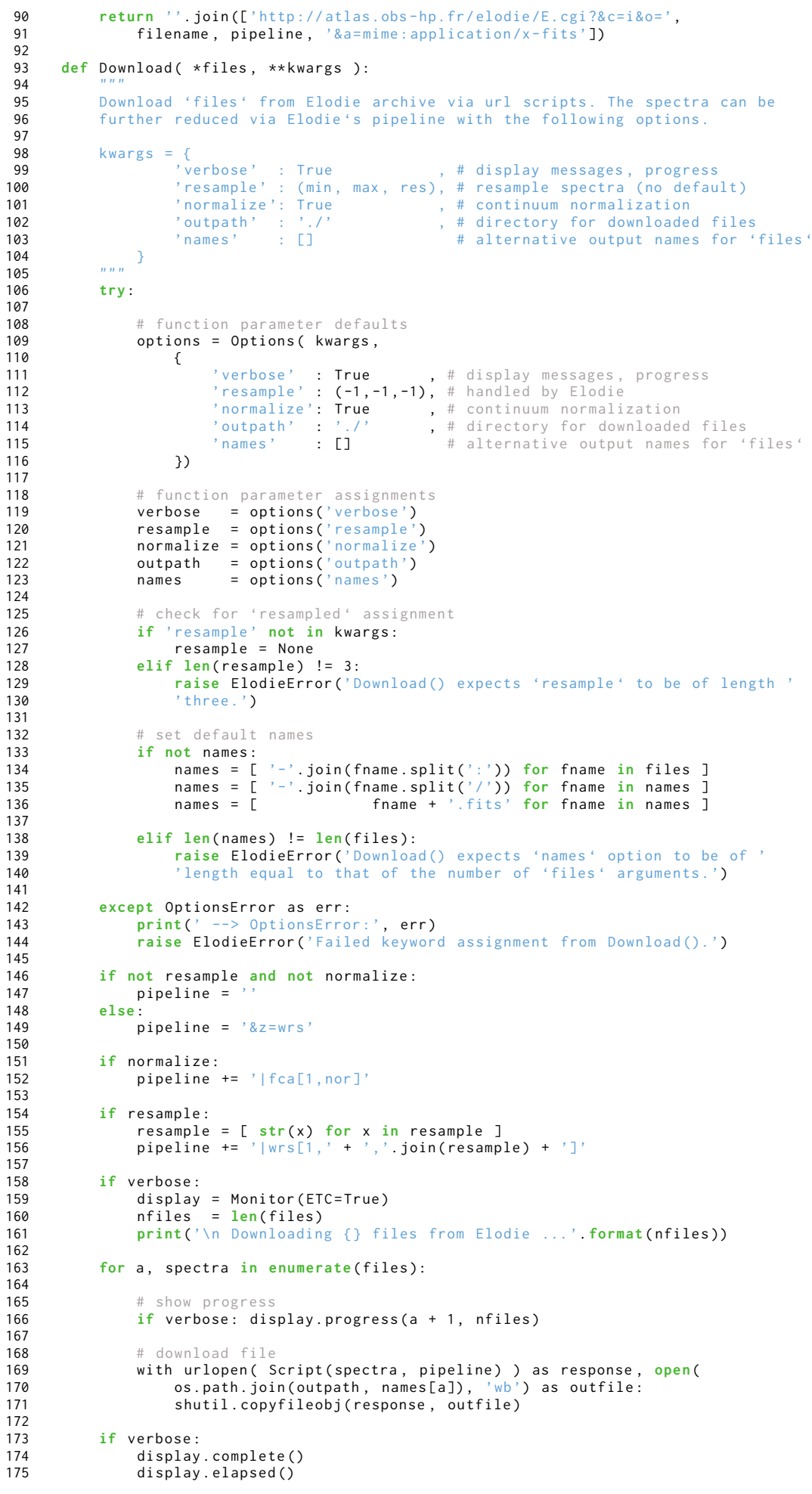




\section{C.6 .. Framework. Argument}

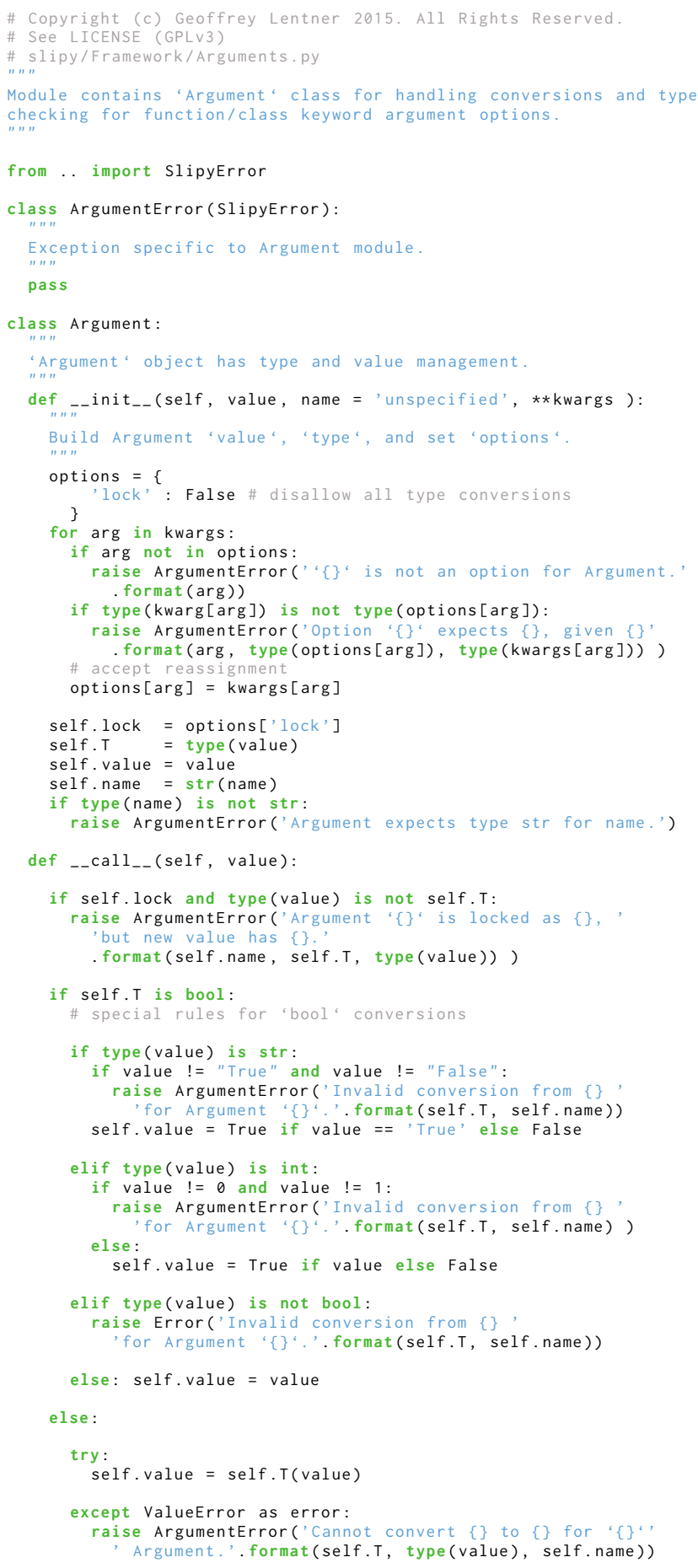




\section{C.7 .. Framework . Command}

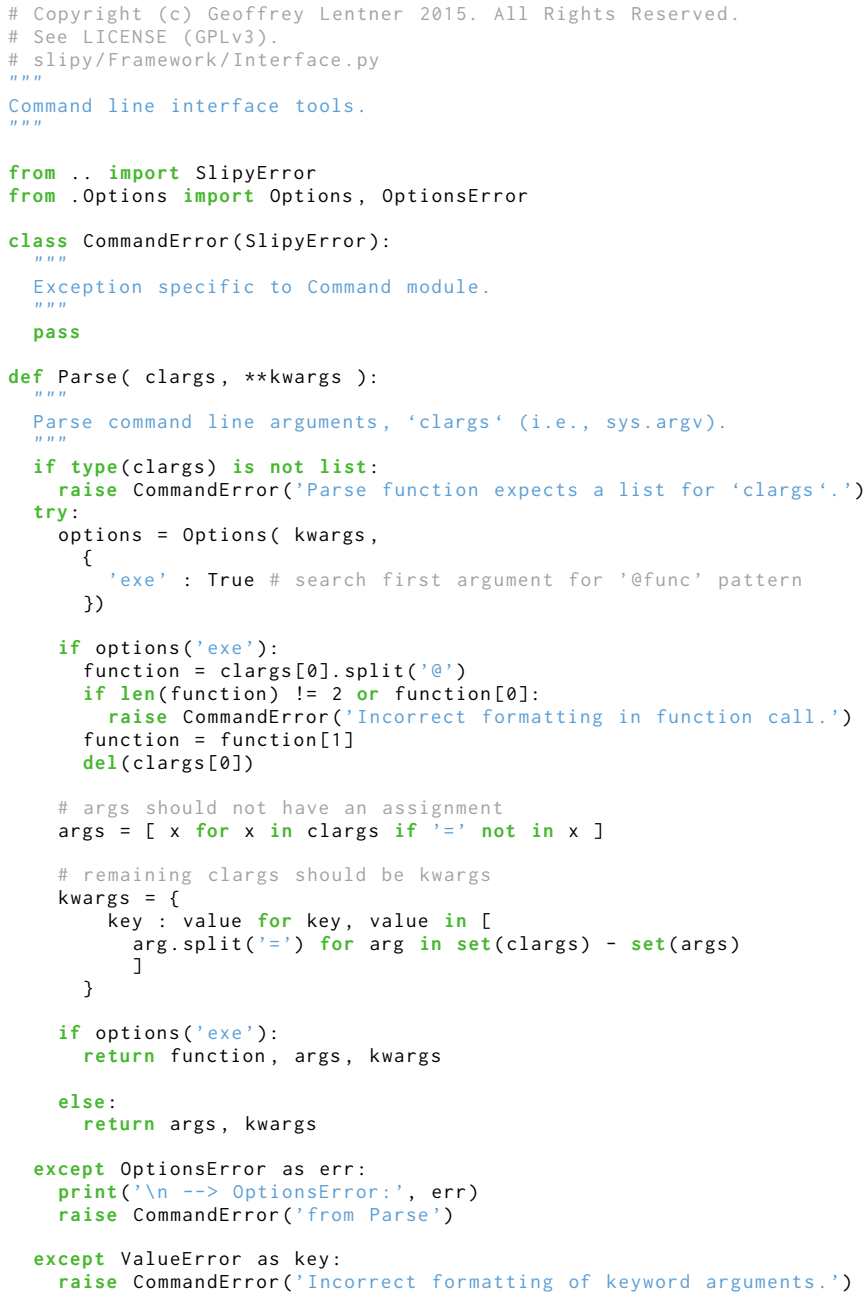




\section{C.8 .. Framework . Display}

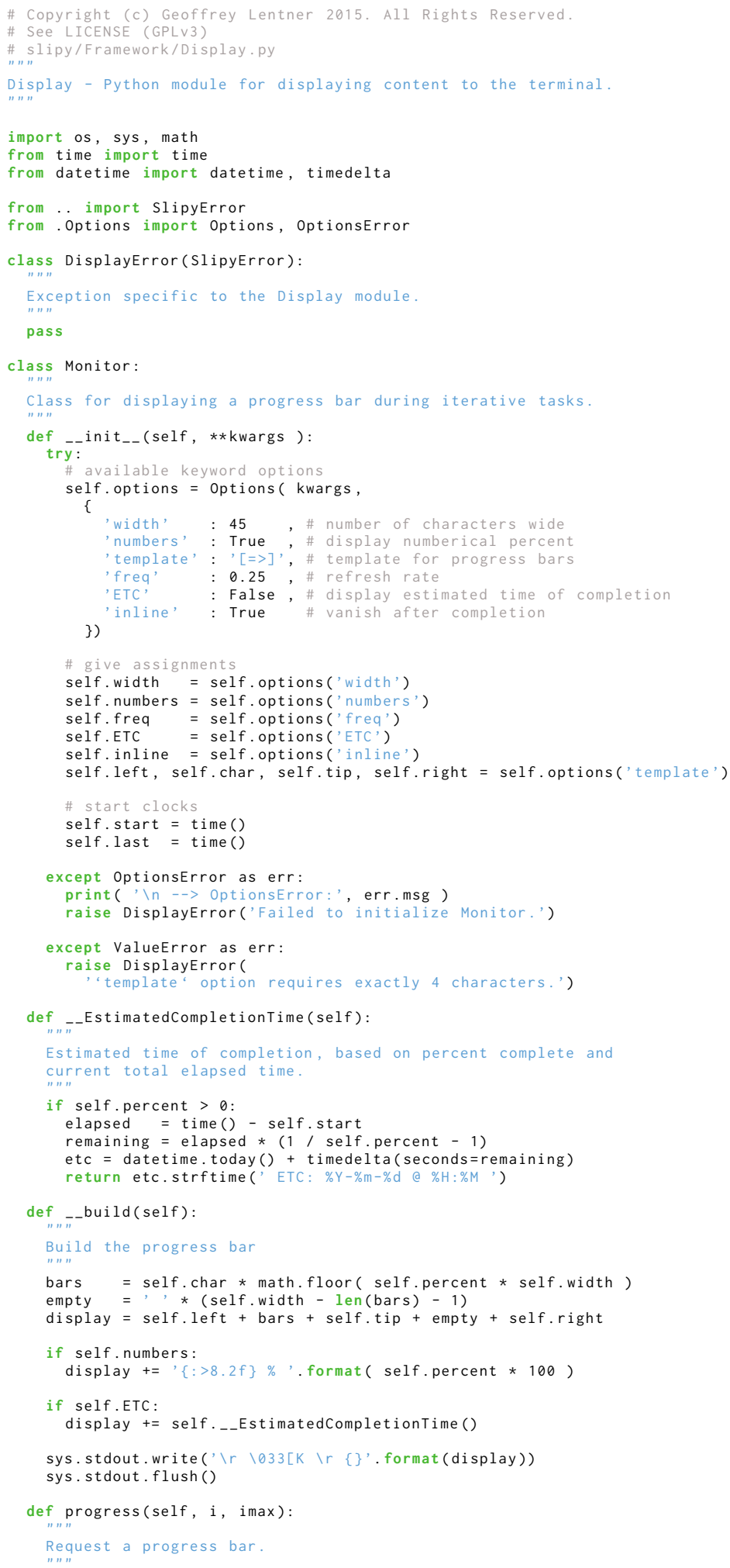




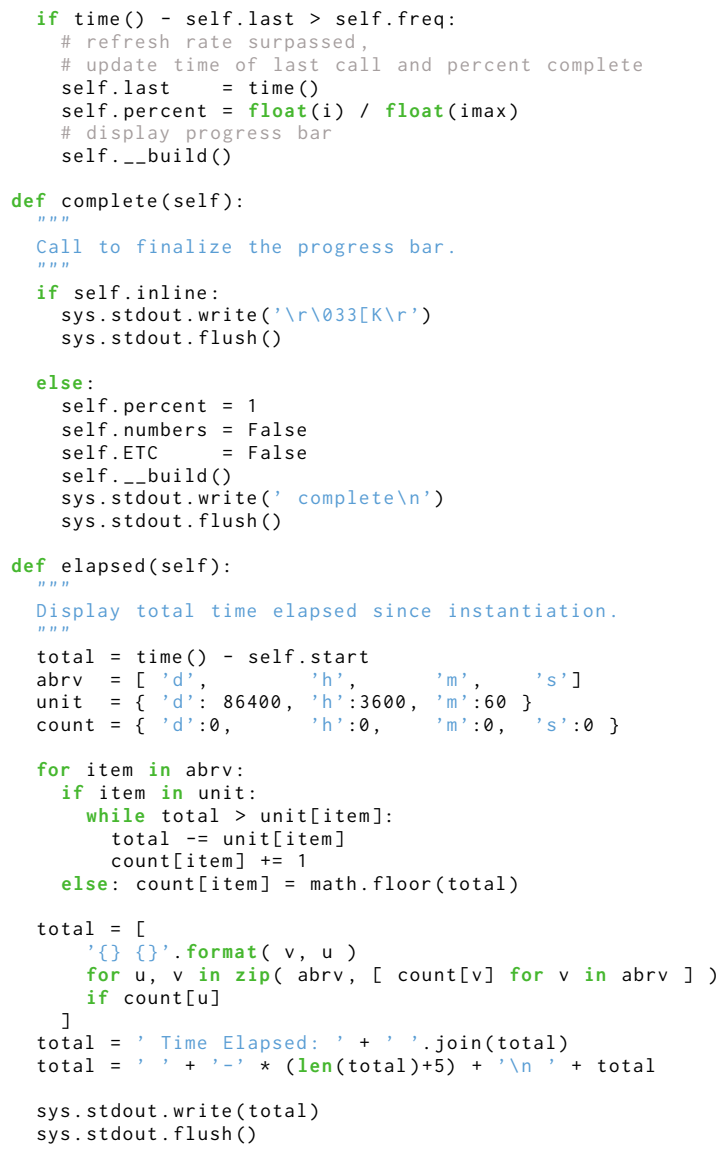




\section{C.9 .. Framework . Measurement}

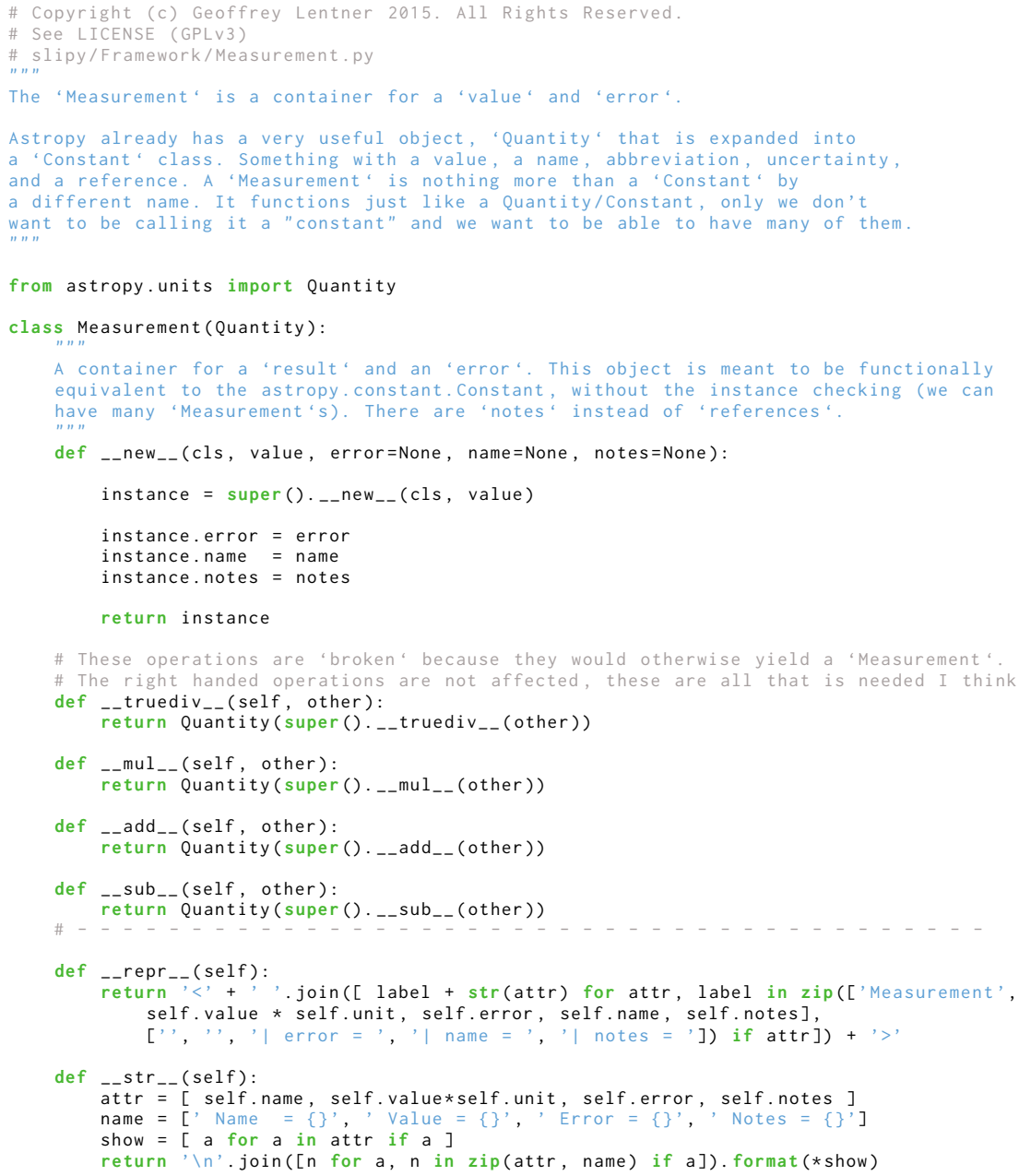




\section{C.10 .. Framework . Options}

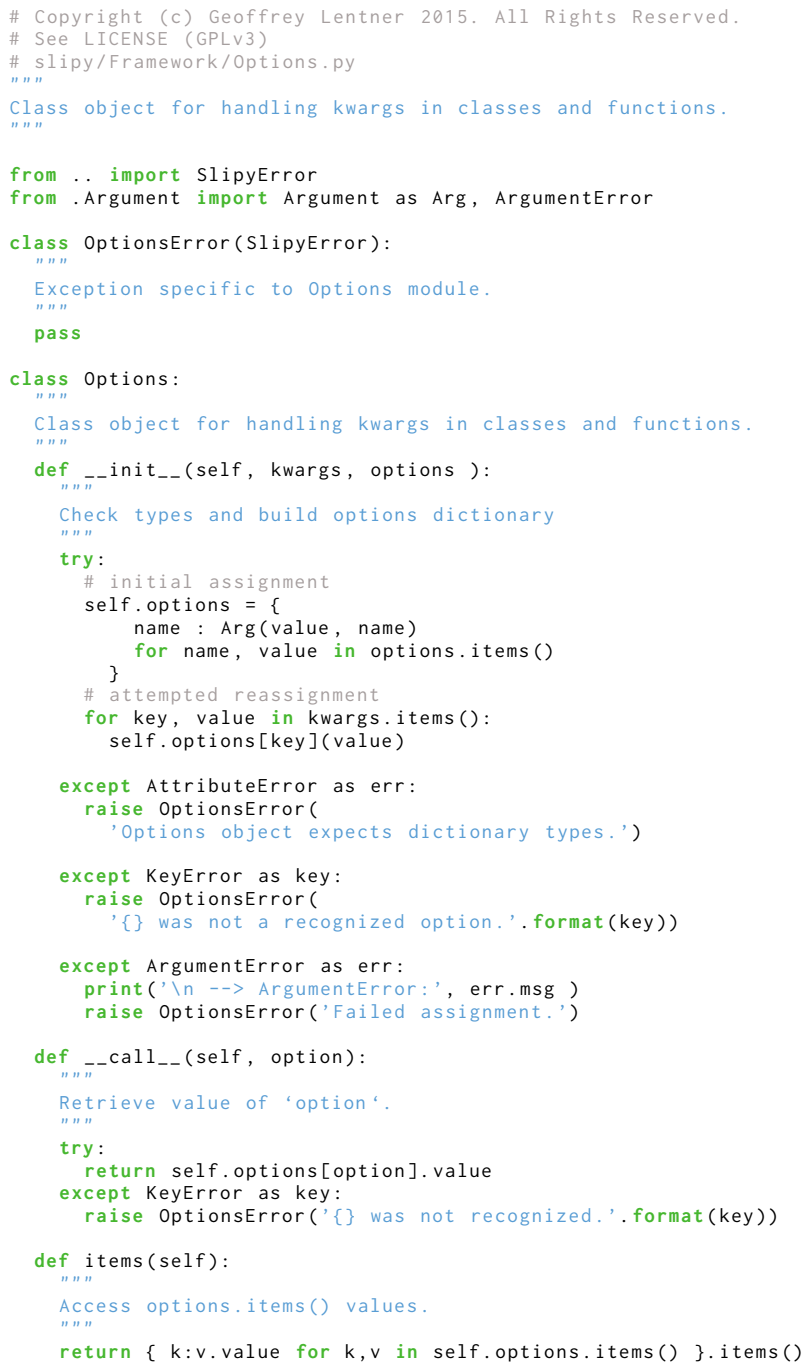




\section{C.11 .. SLiPy . Correlate}

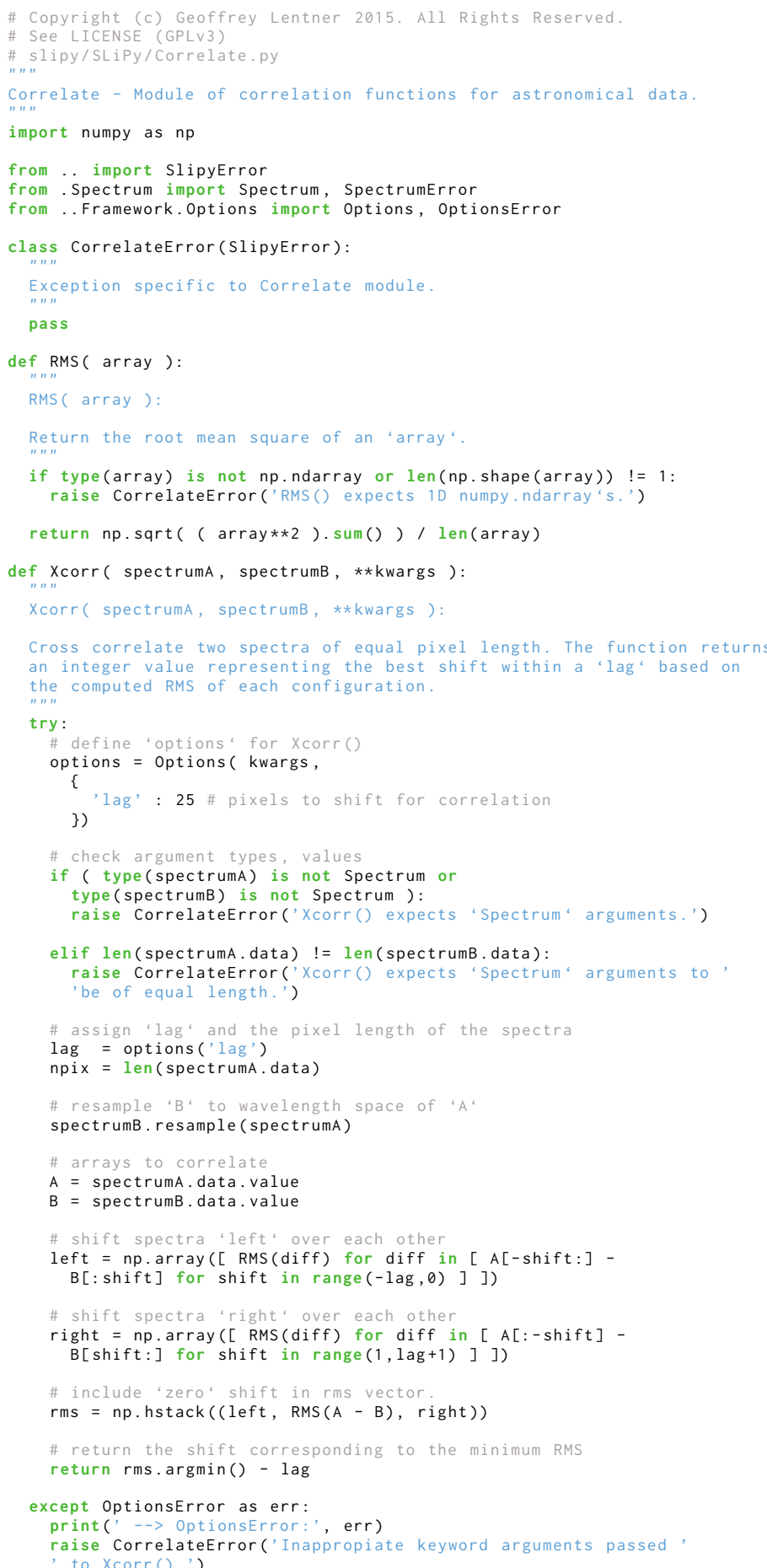




\section{C.12 .. SLiPy . Fits}

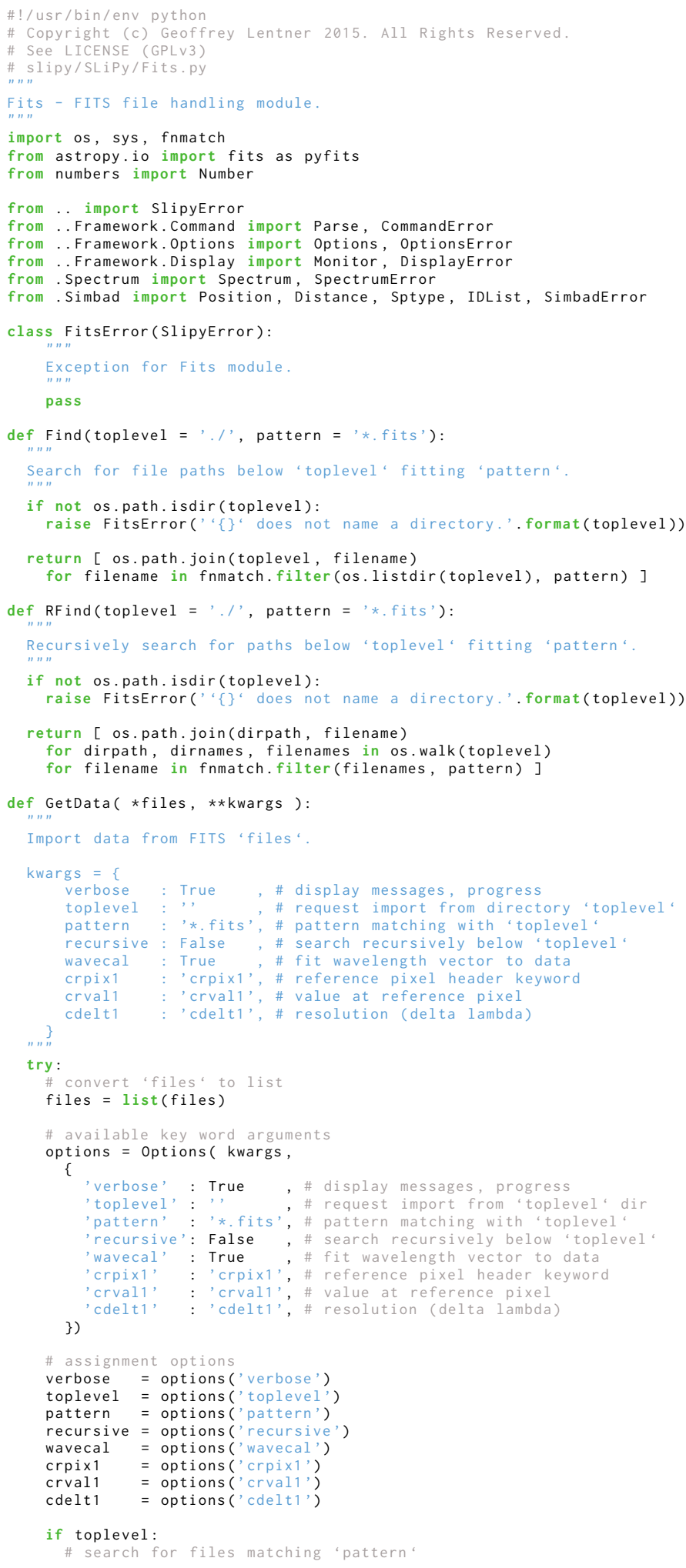




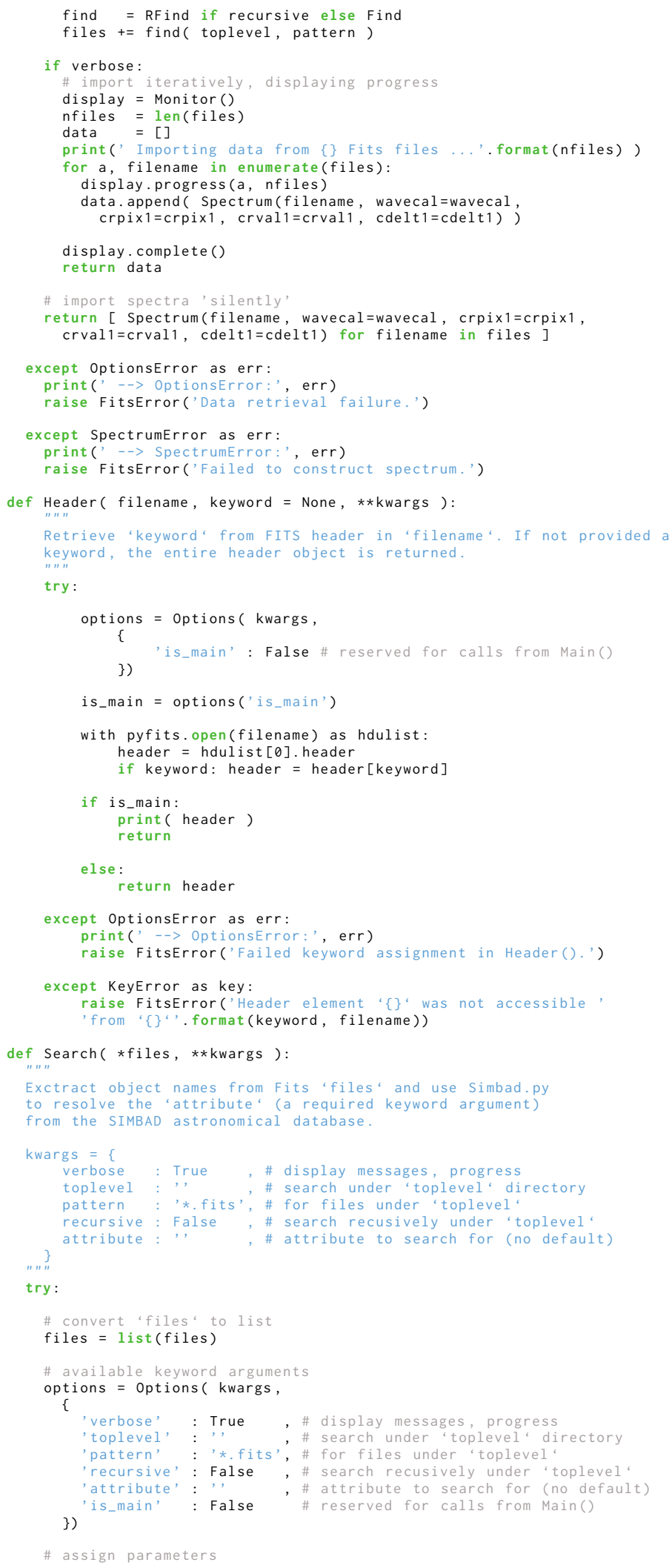




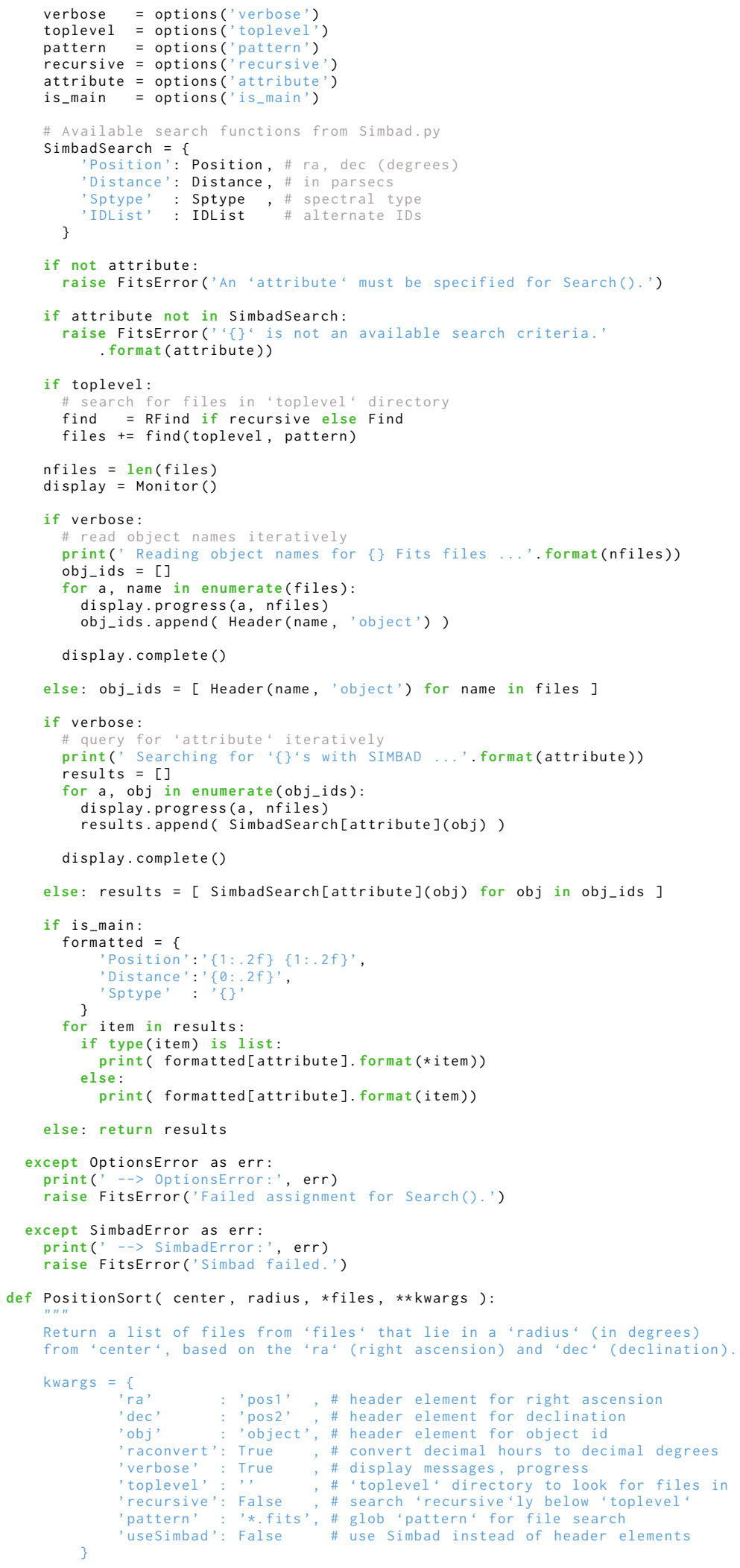




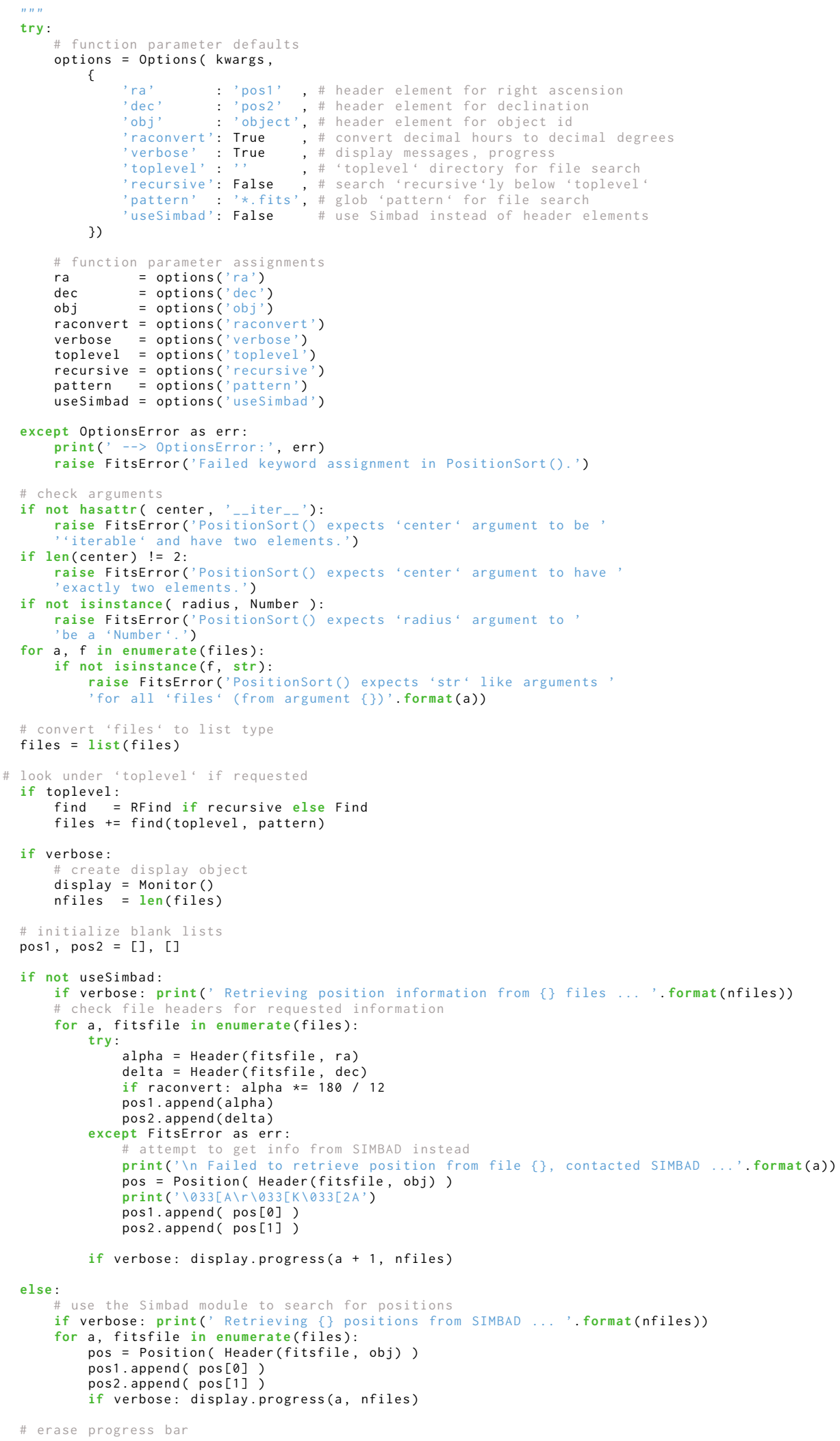




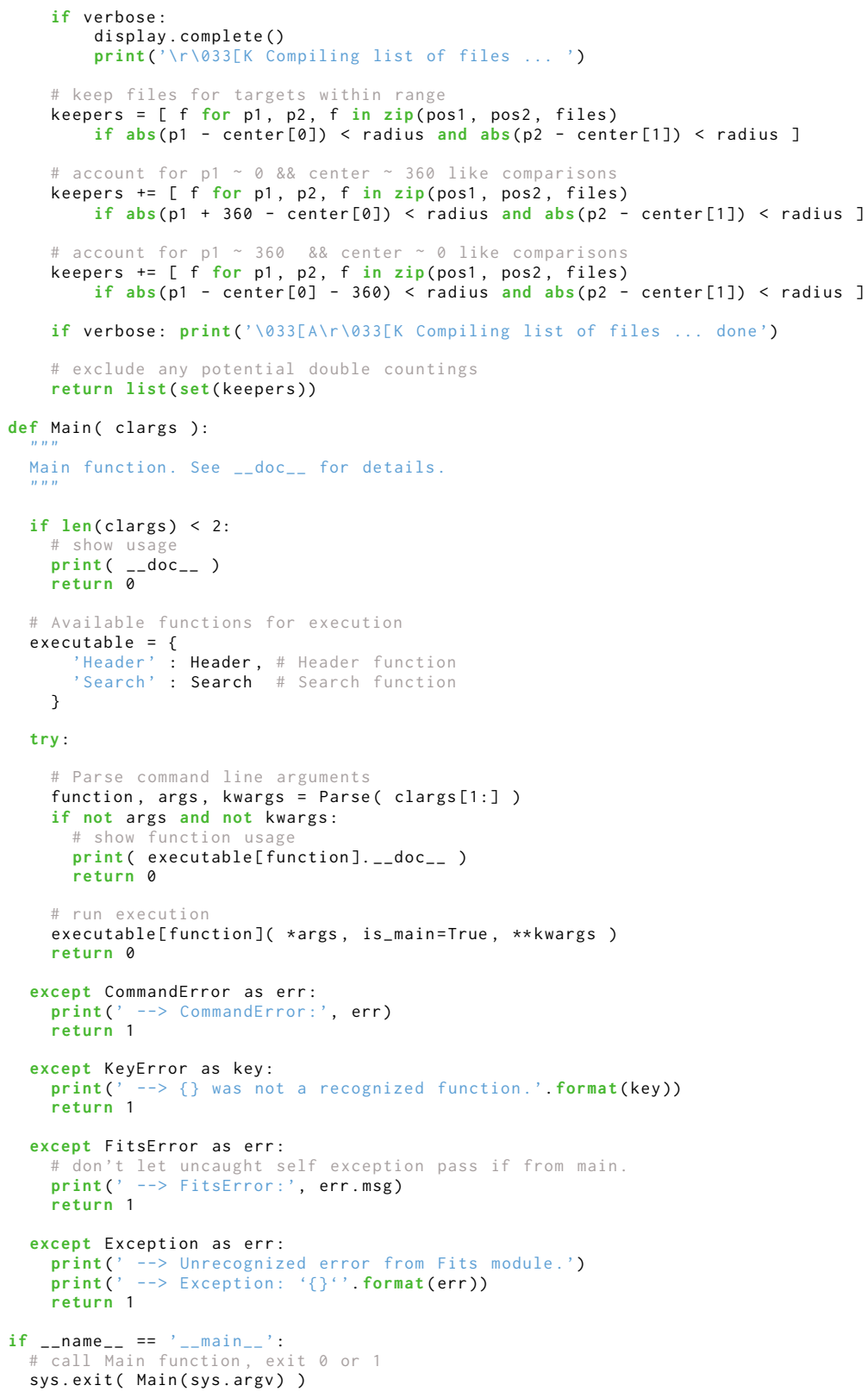




\section{C.13 .. SLiPy . Montage}

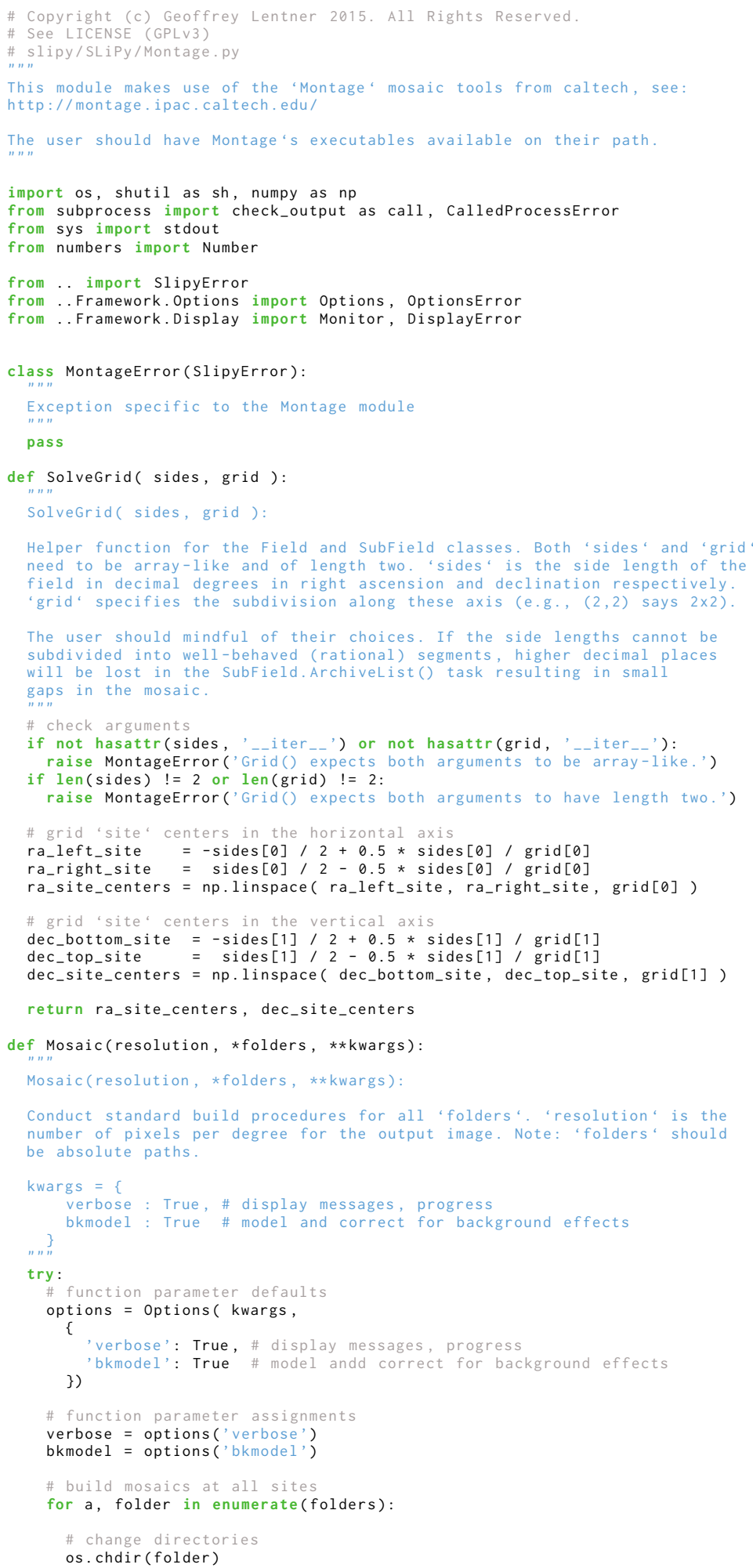




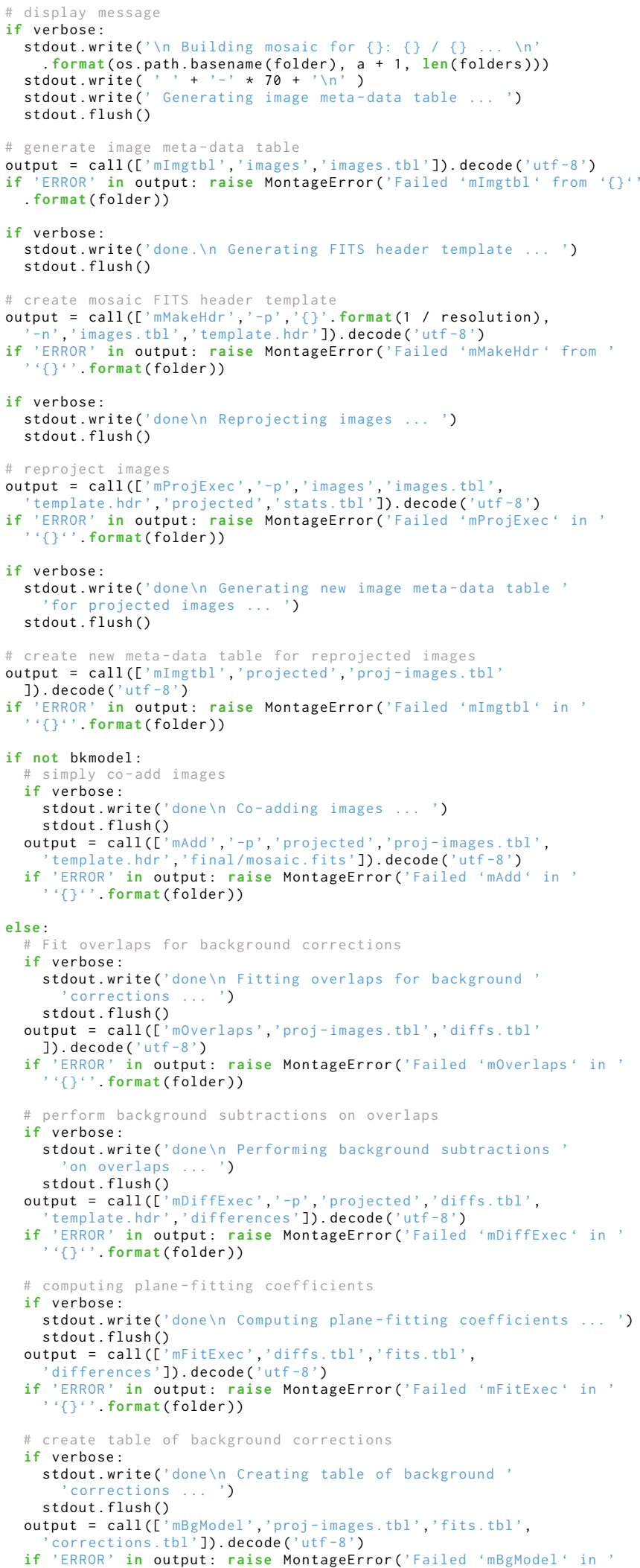




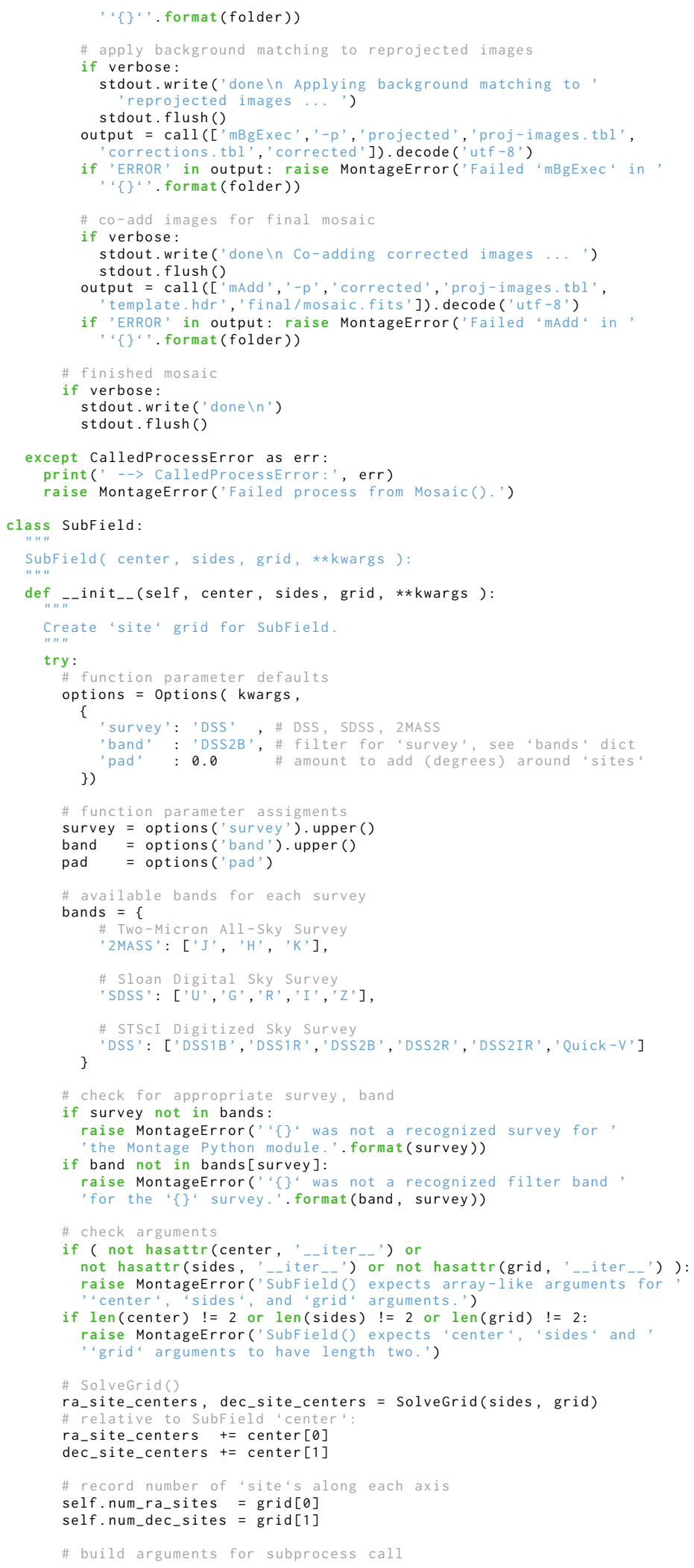




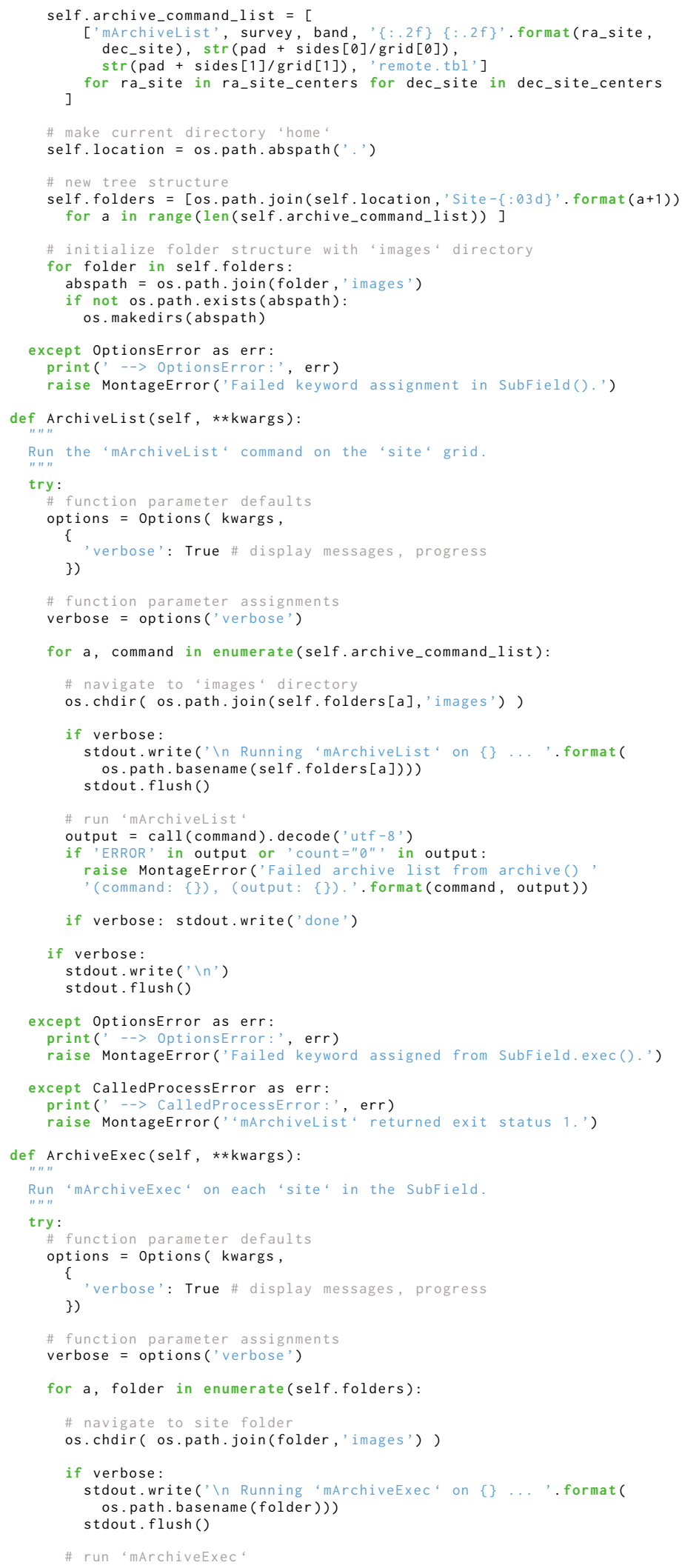




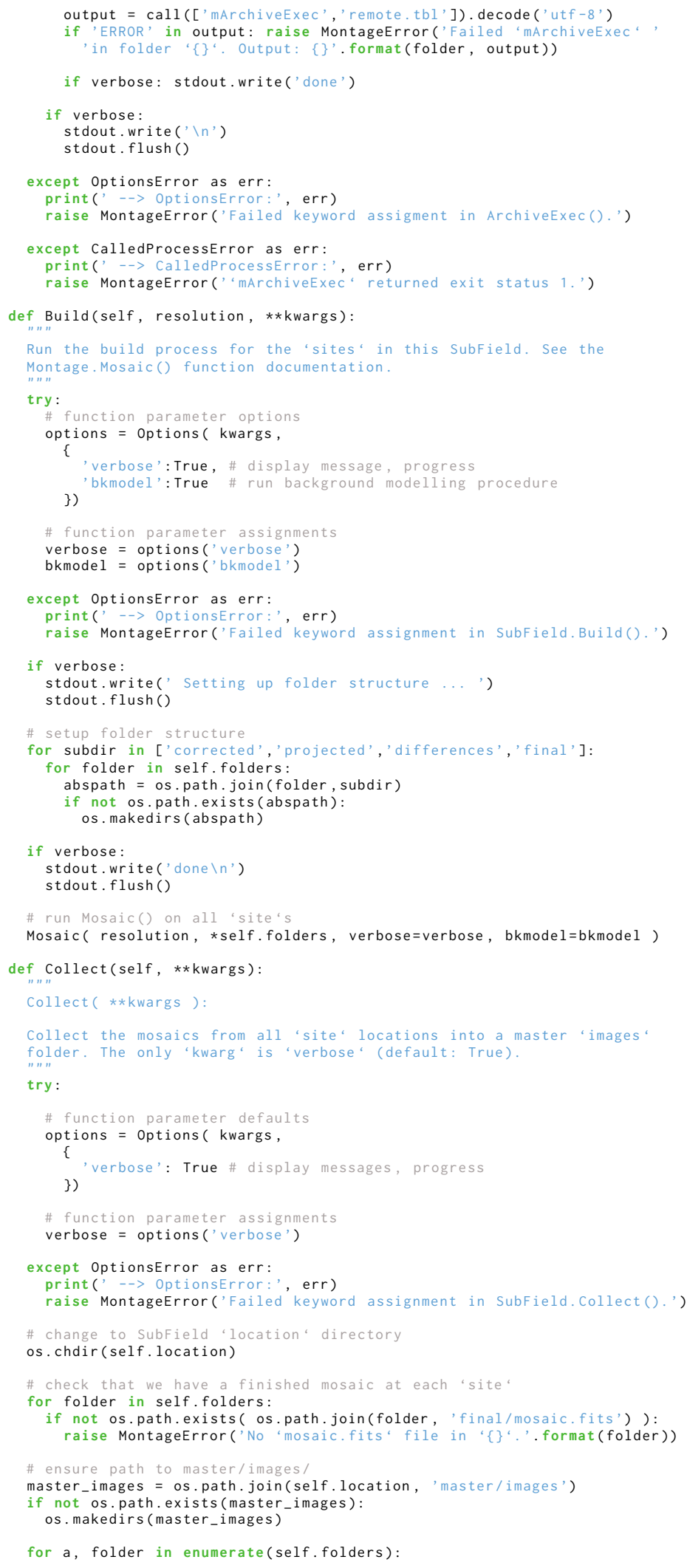




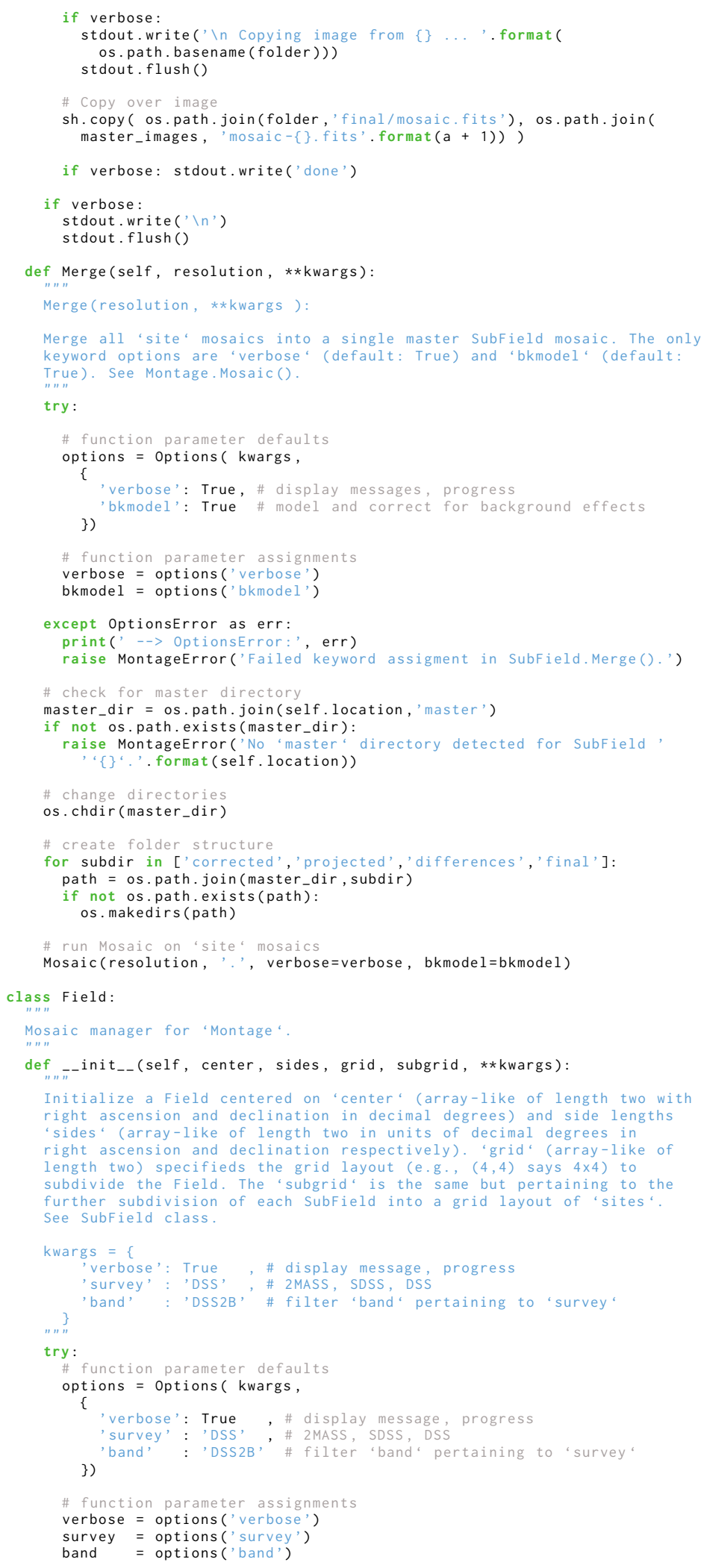




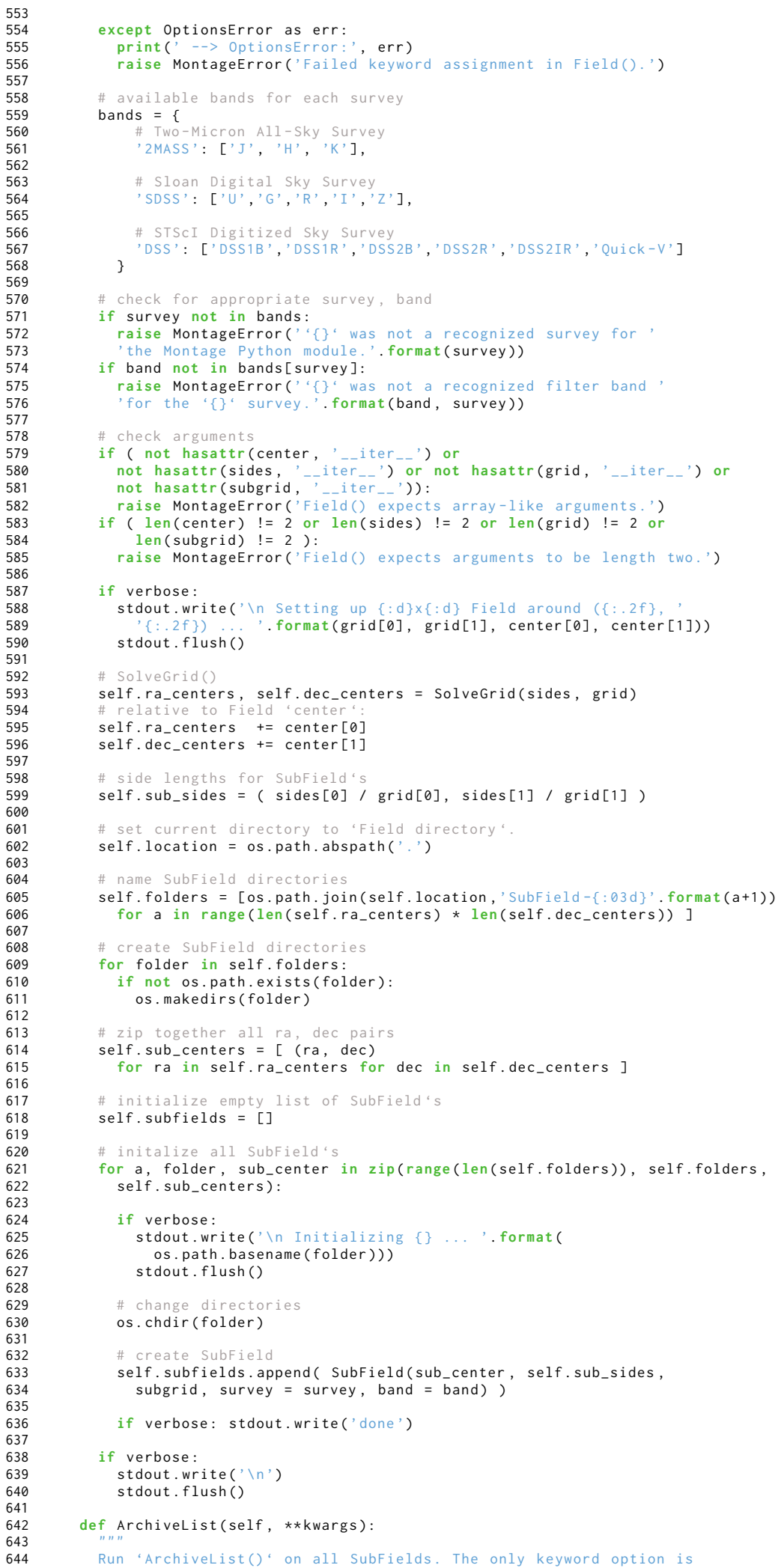




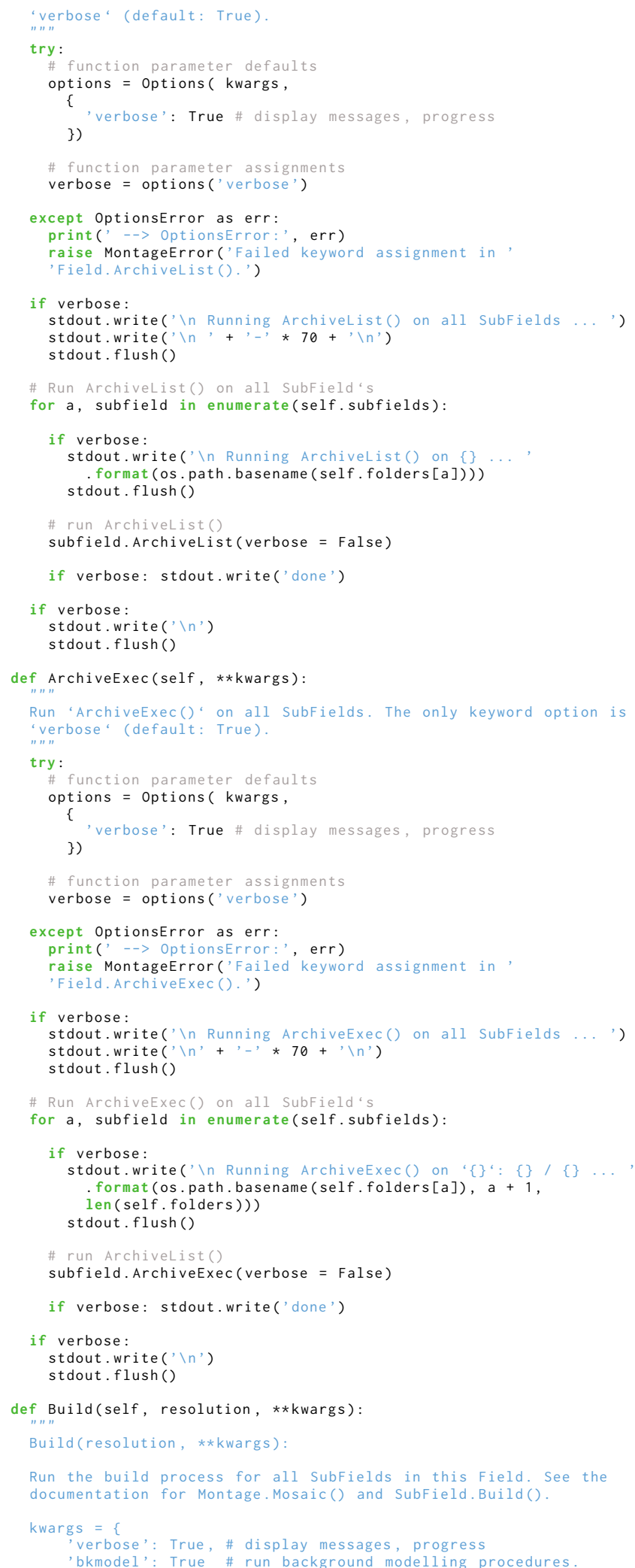




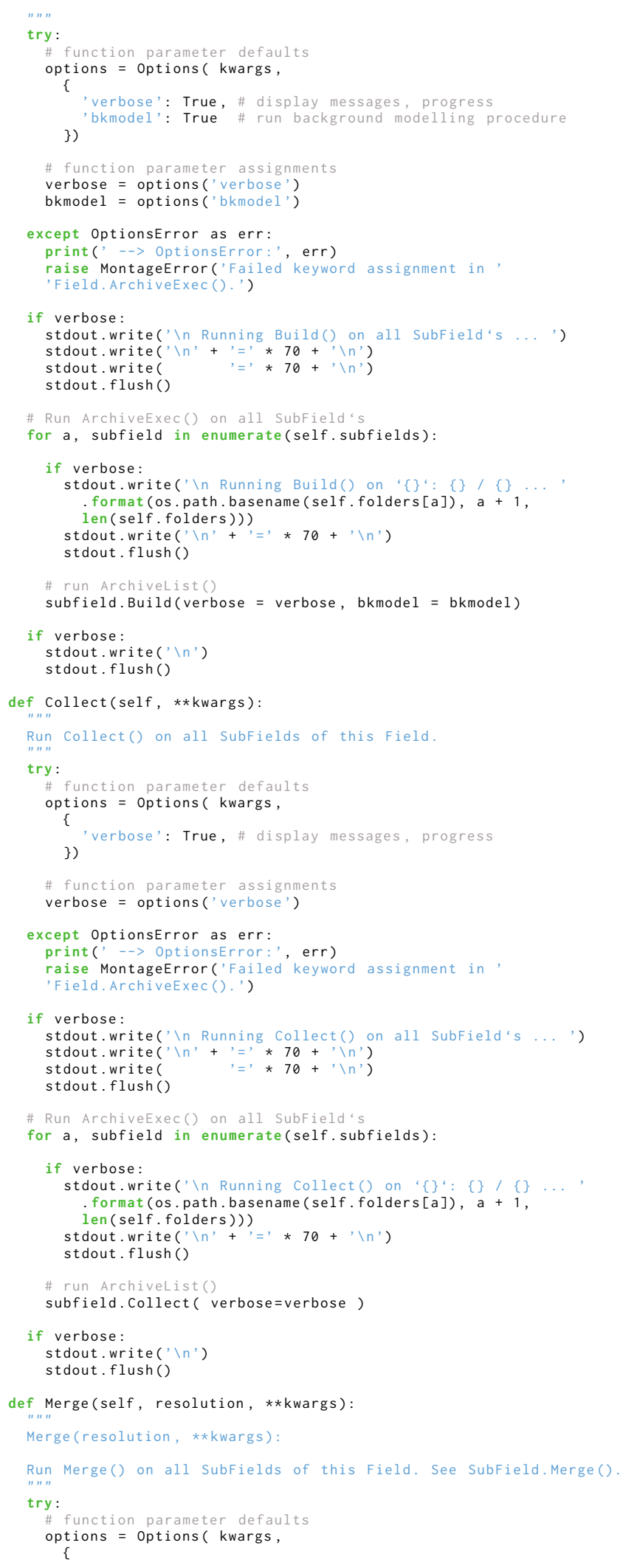




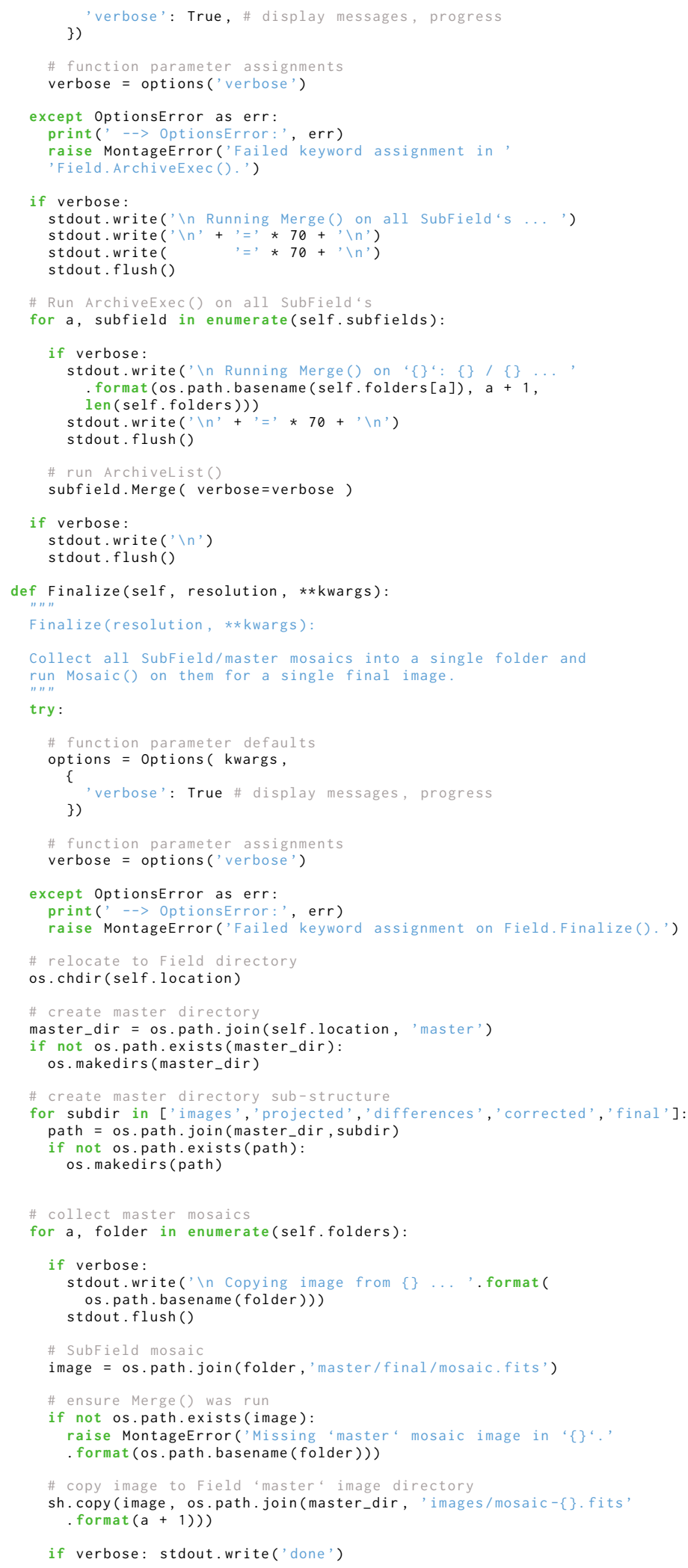


if verbose:

stdout. write (' $\backslash$ n')

stdout.flush()

\# change directories to "master

os.chdir(master_dir)

\# run Mosaic() on all subField 'master's

Mosaic (resolution, ', ', verbose=verbose, bkmodel=bkmodel) 


\section{C.14 .. SLiPy . Observatory}

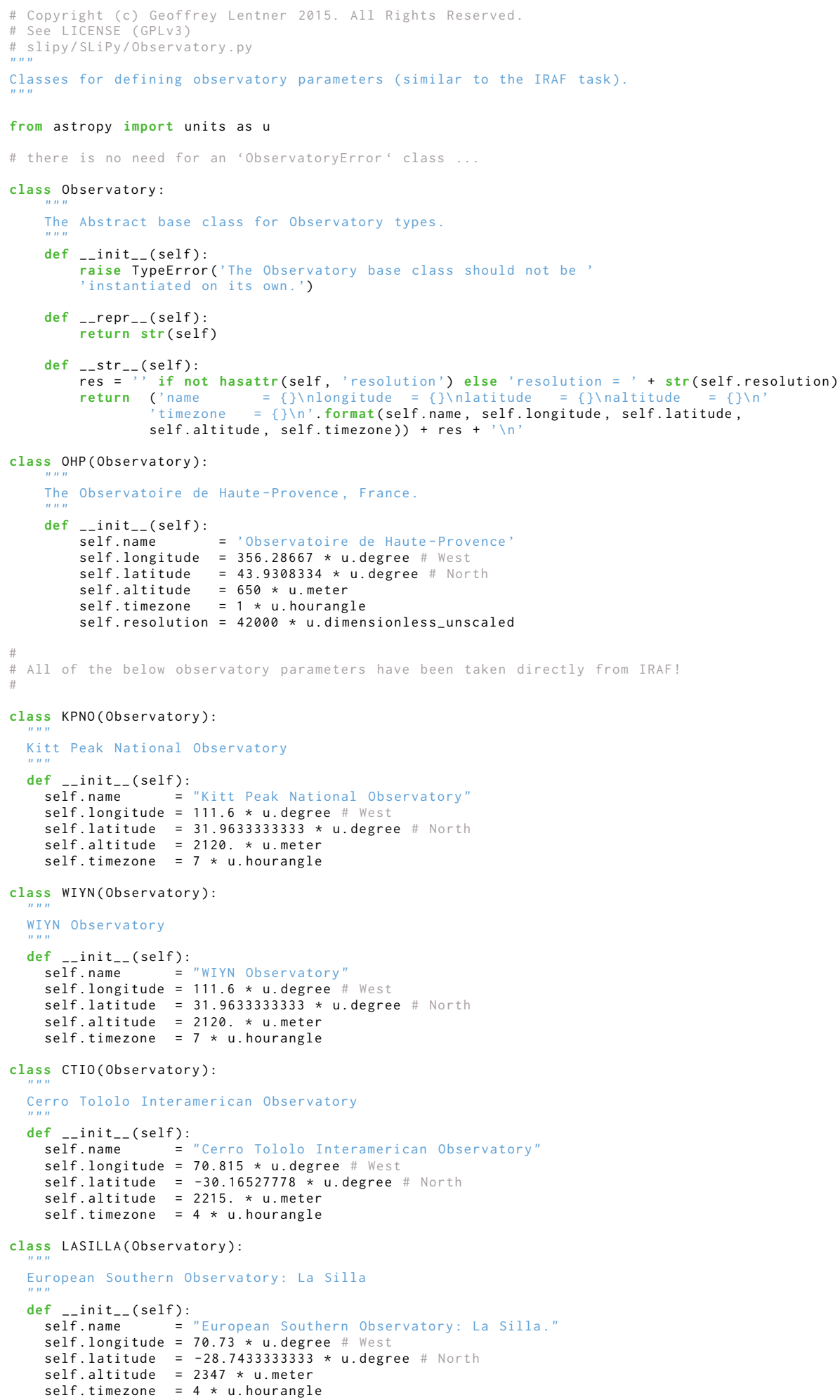




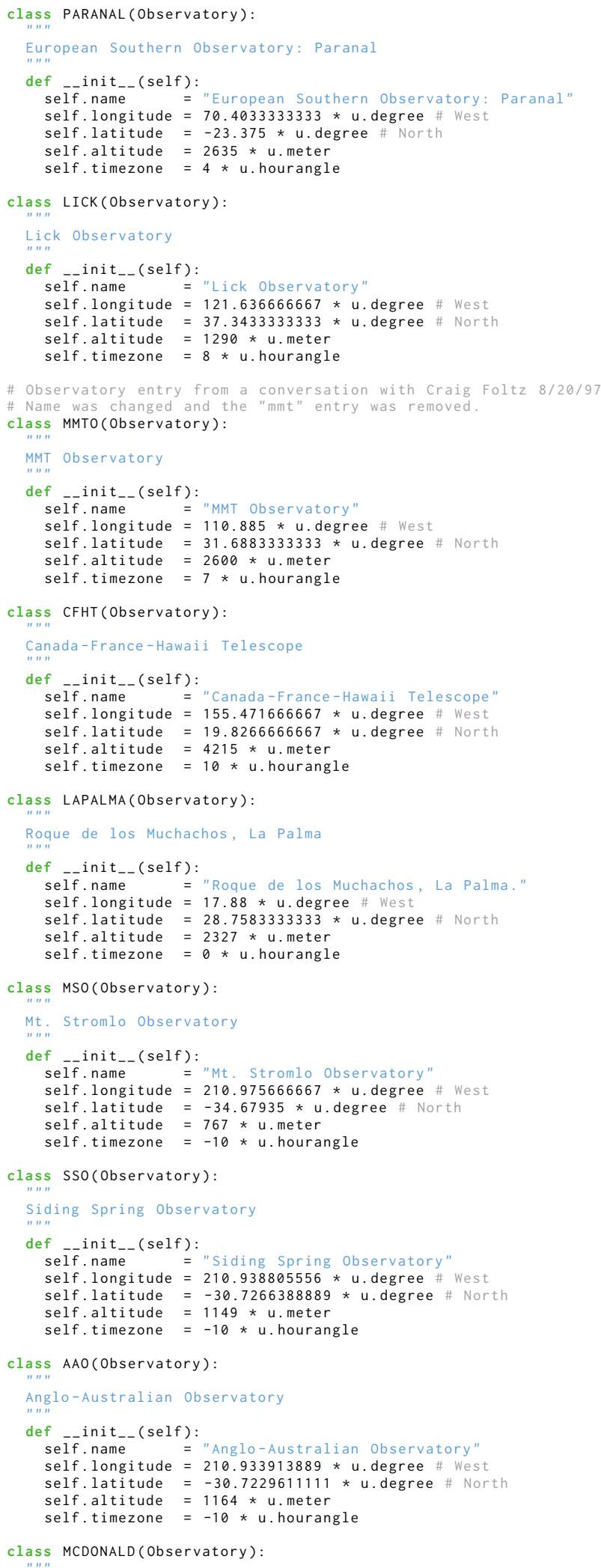




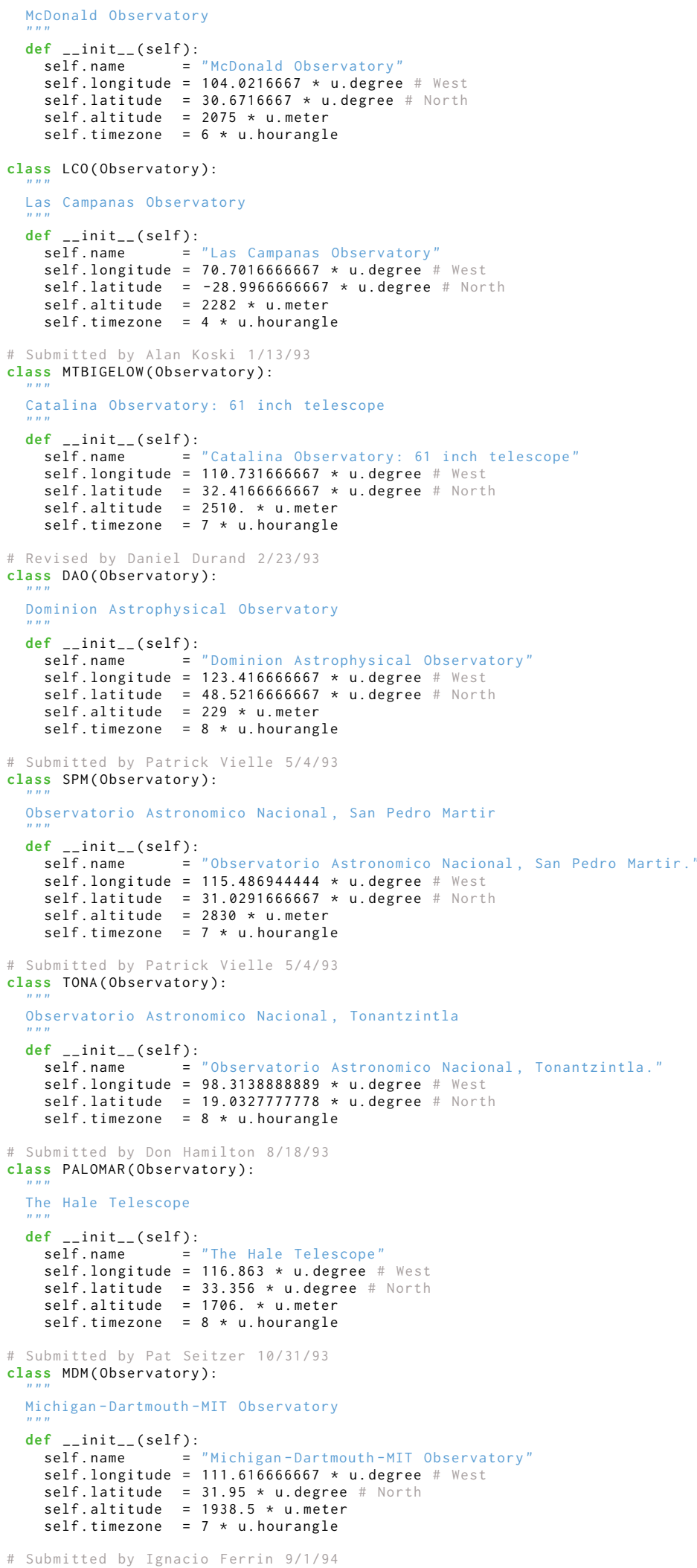




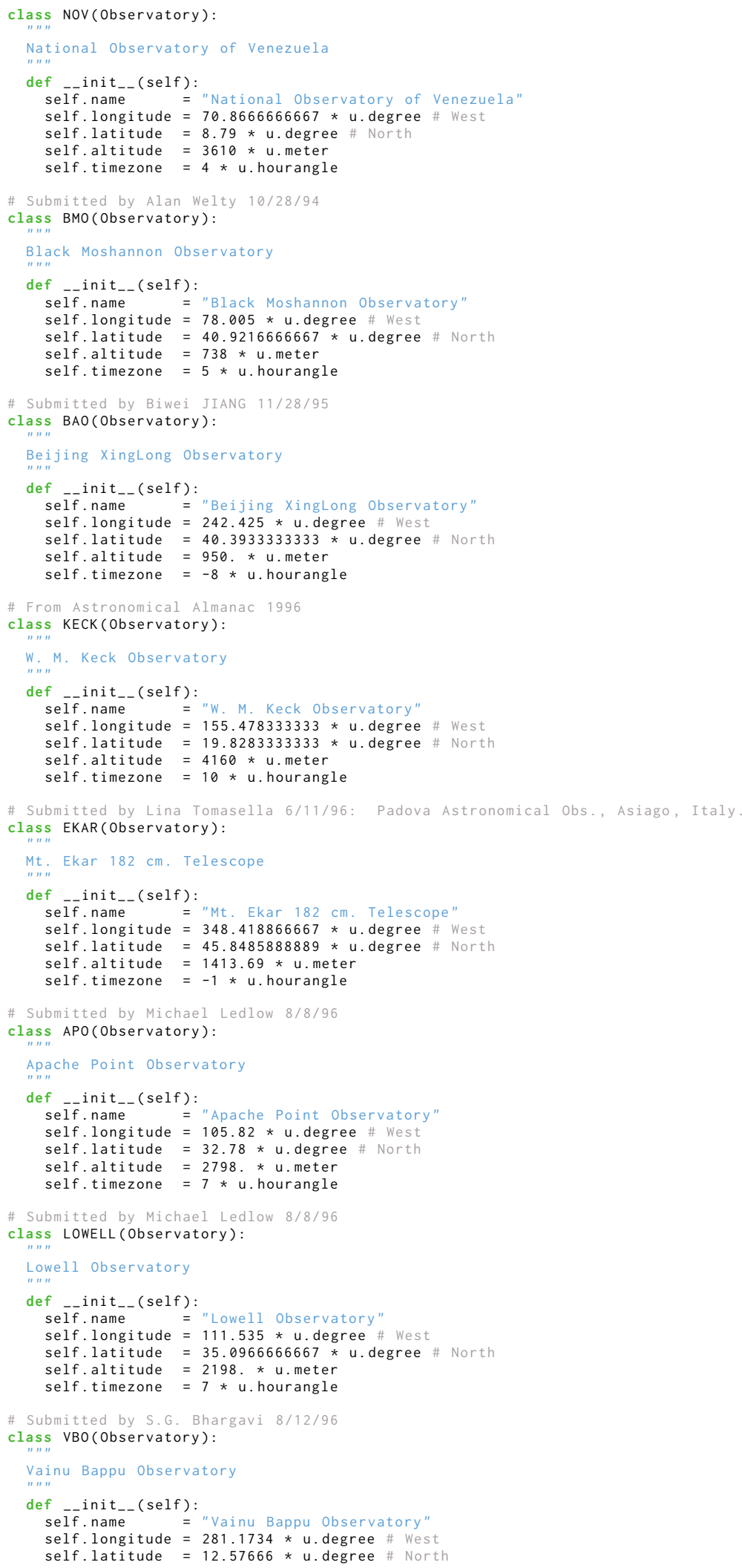




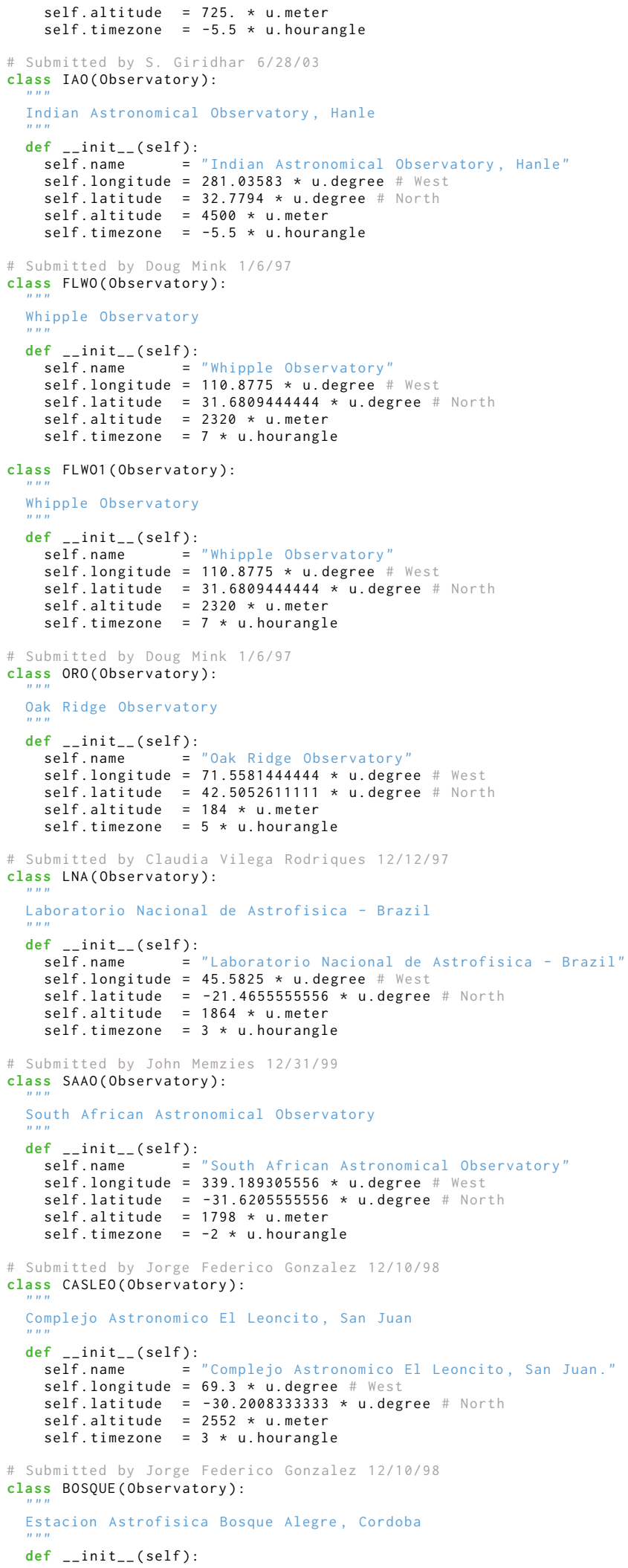




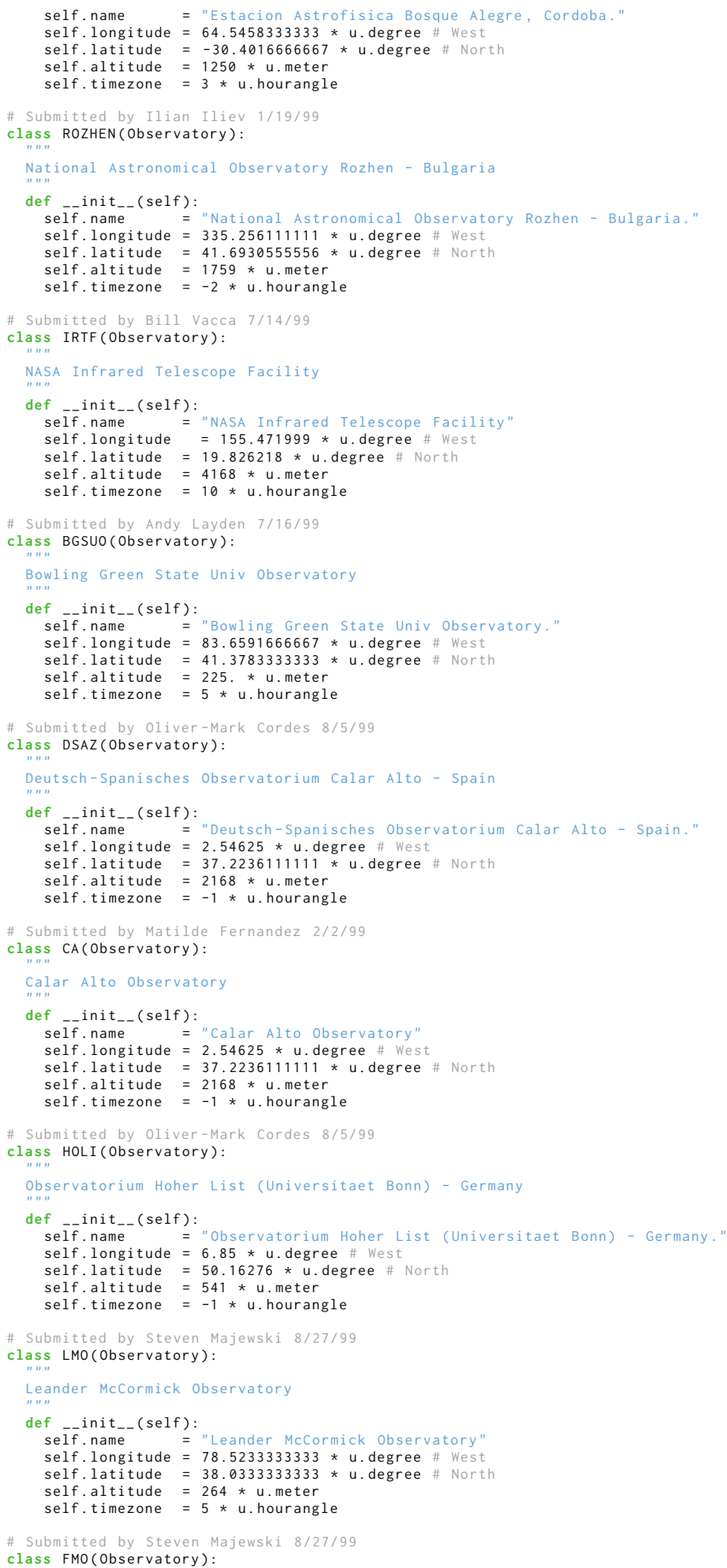




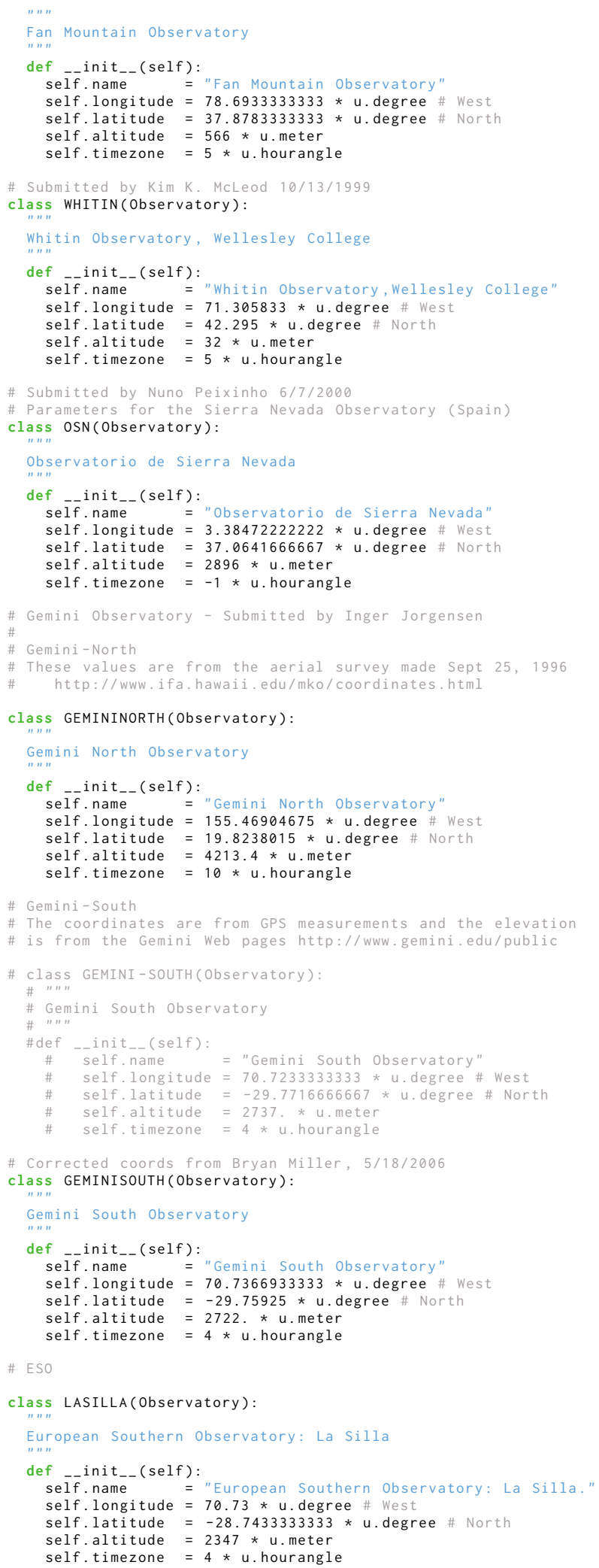




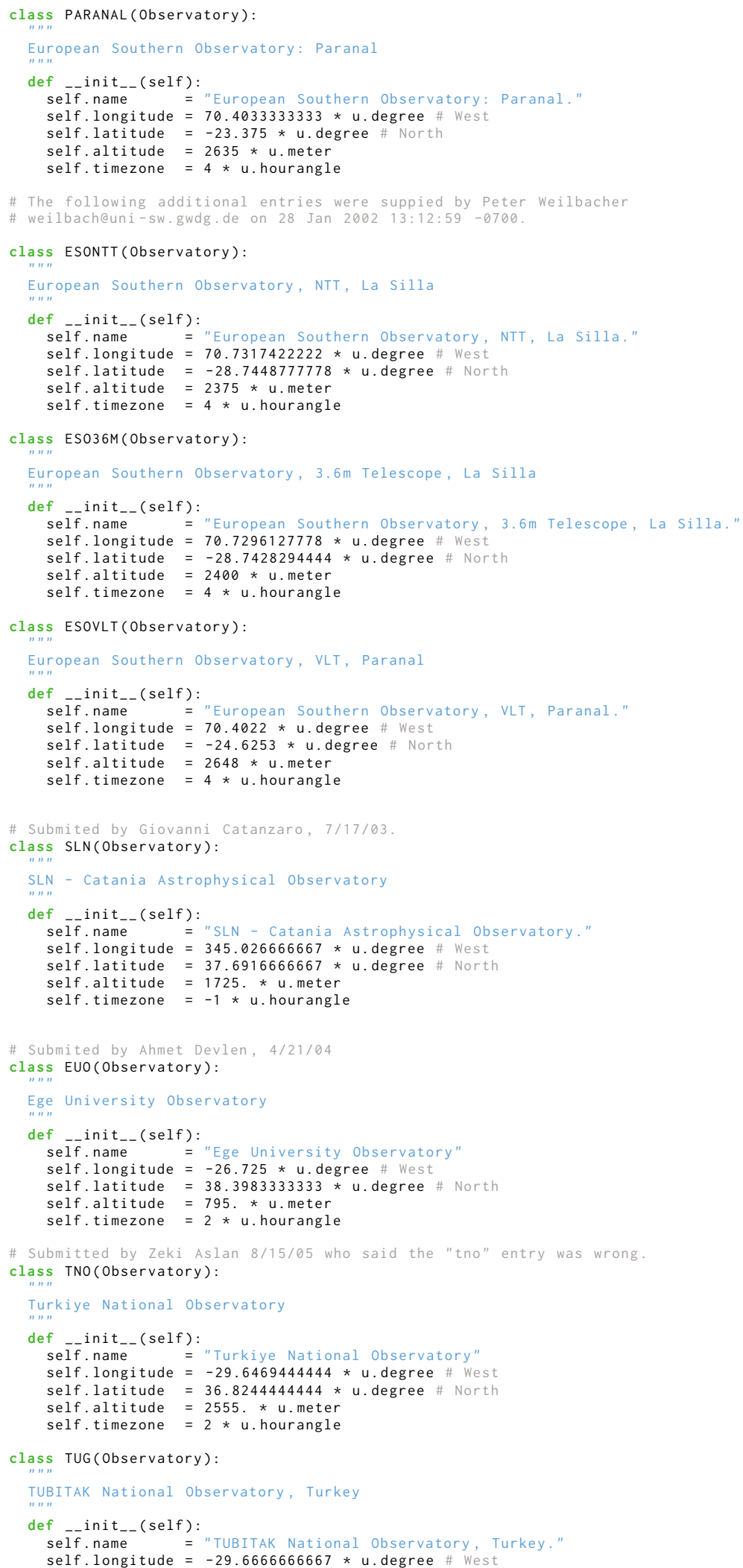




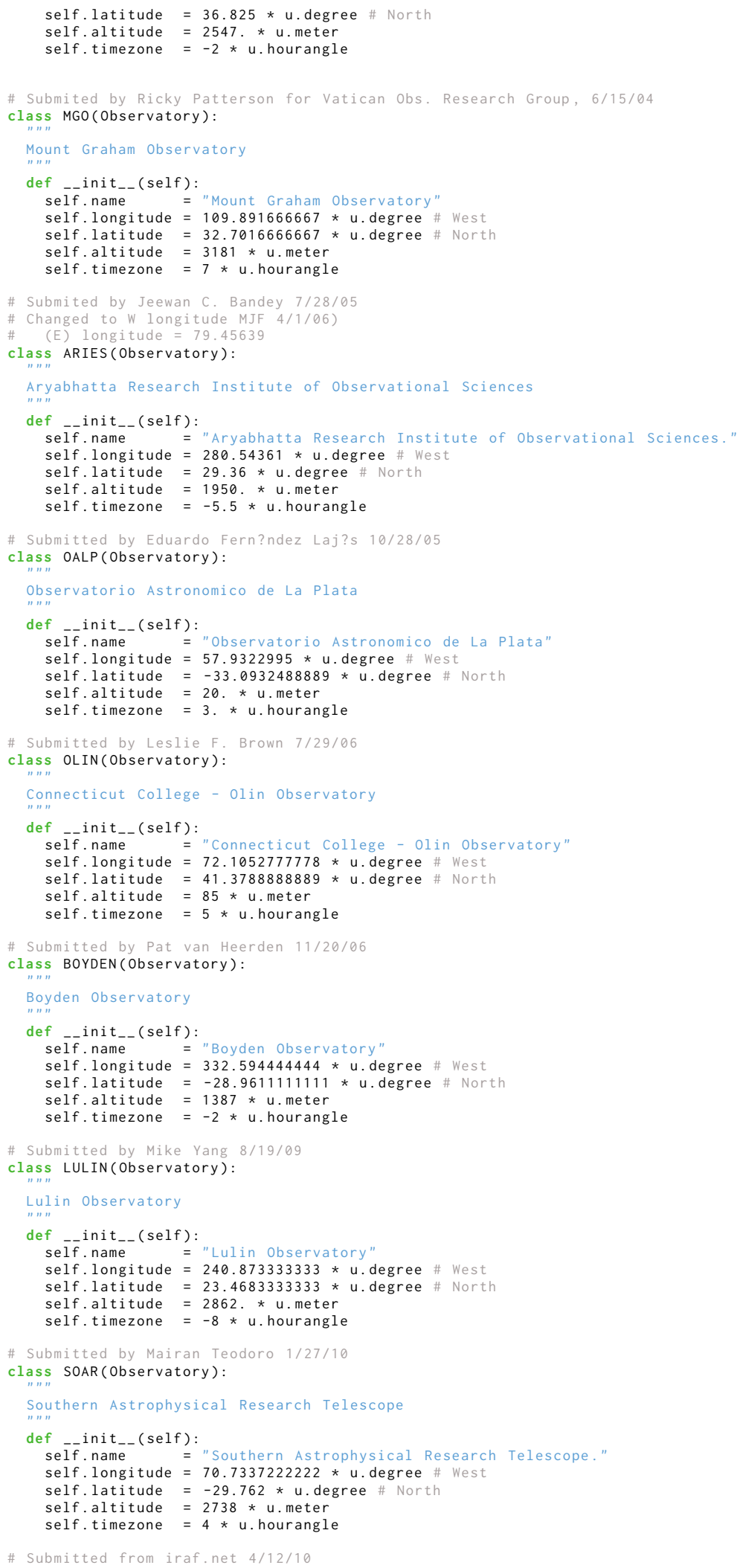




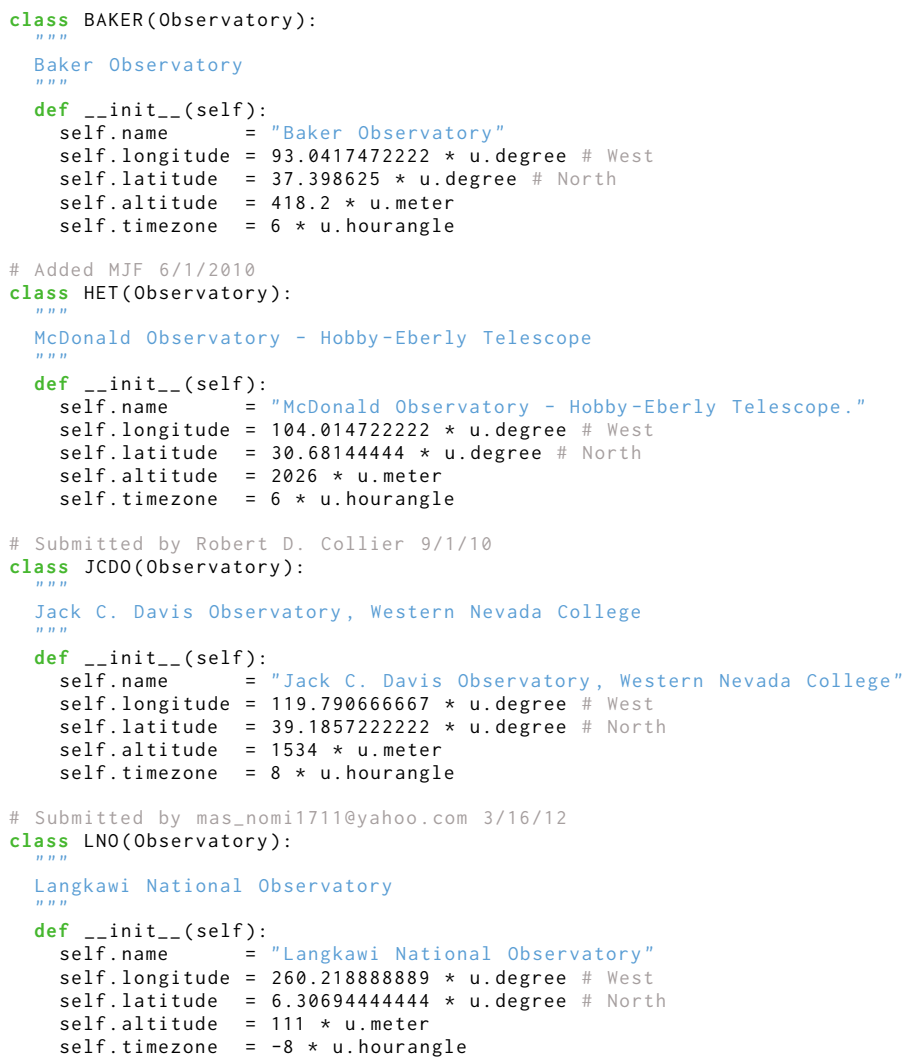




\section{C.15 .. SLiPy . Plot}

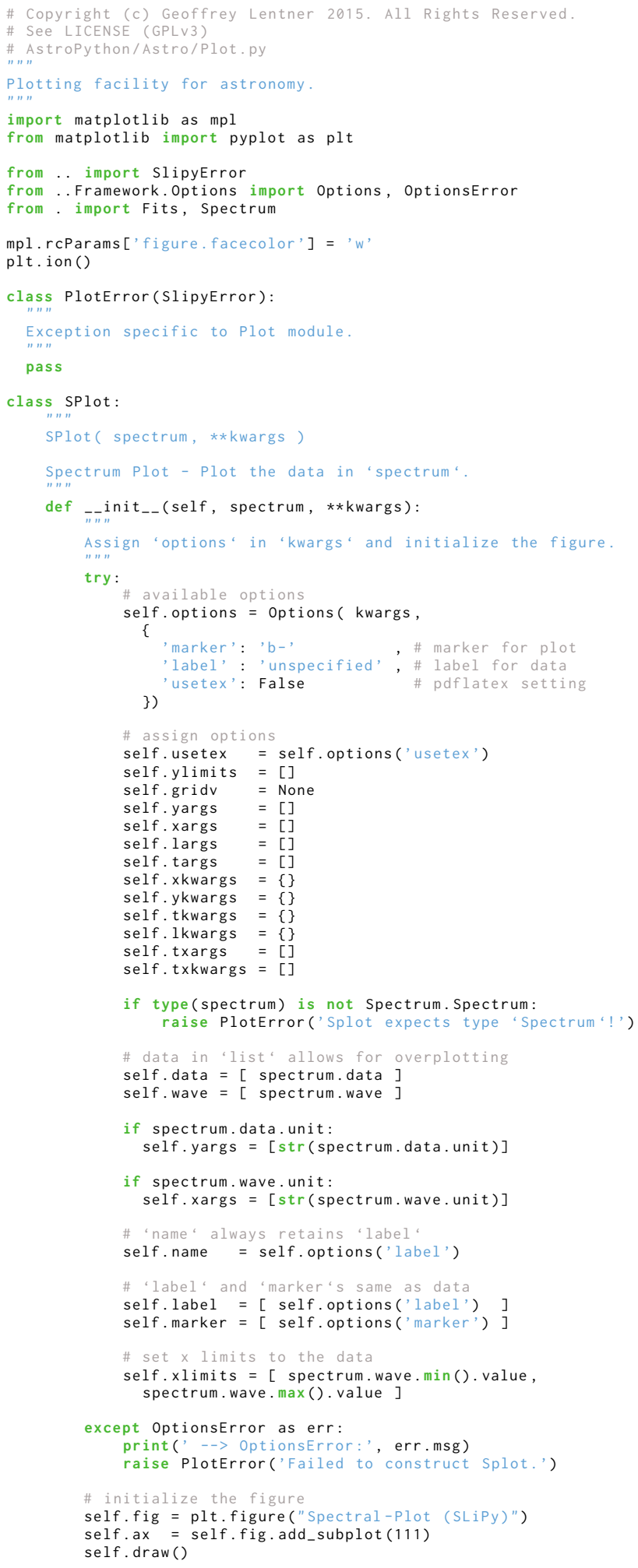




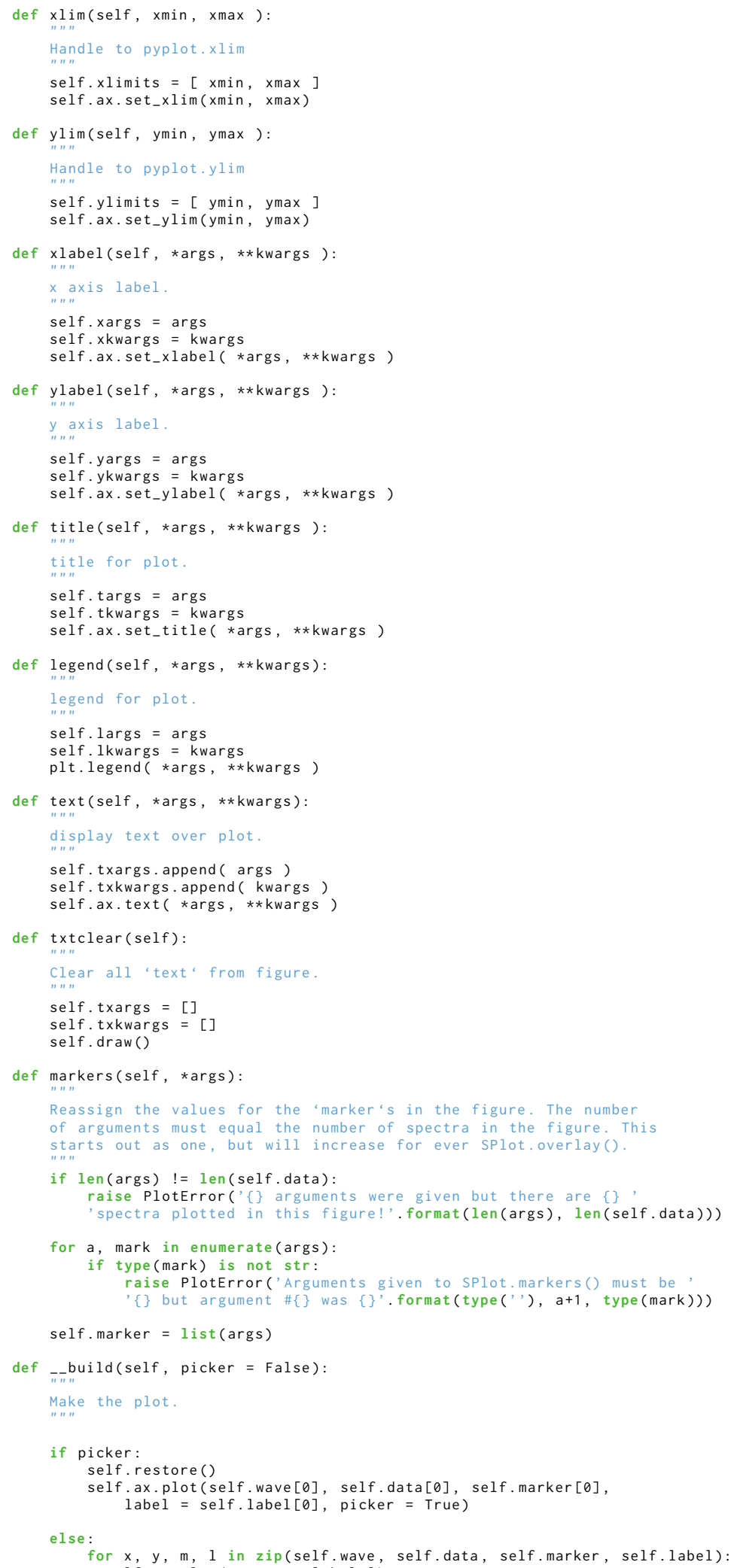




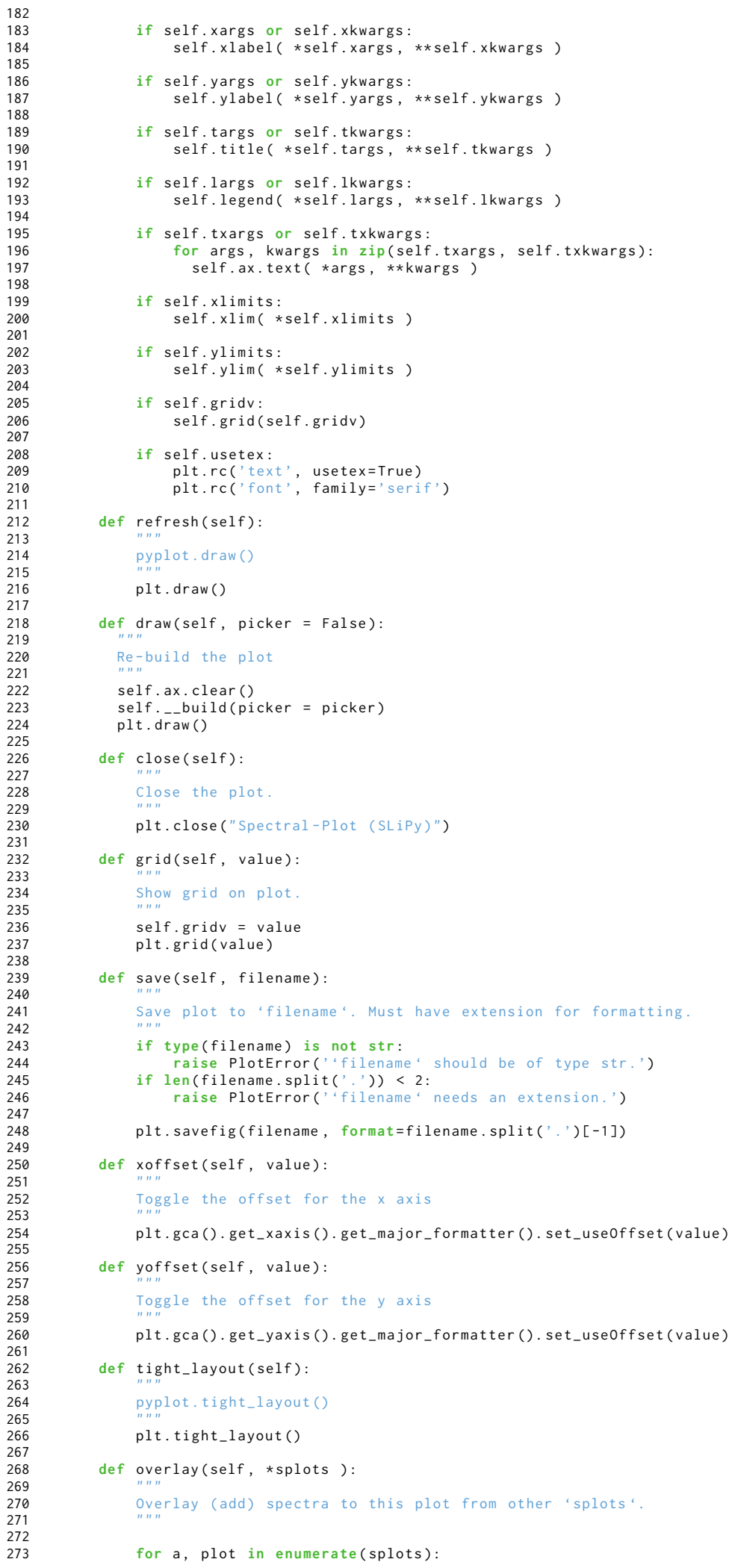




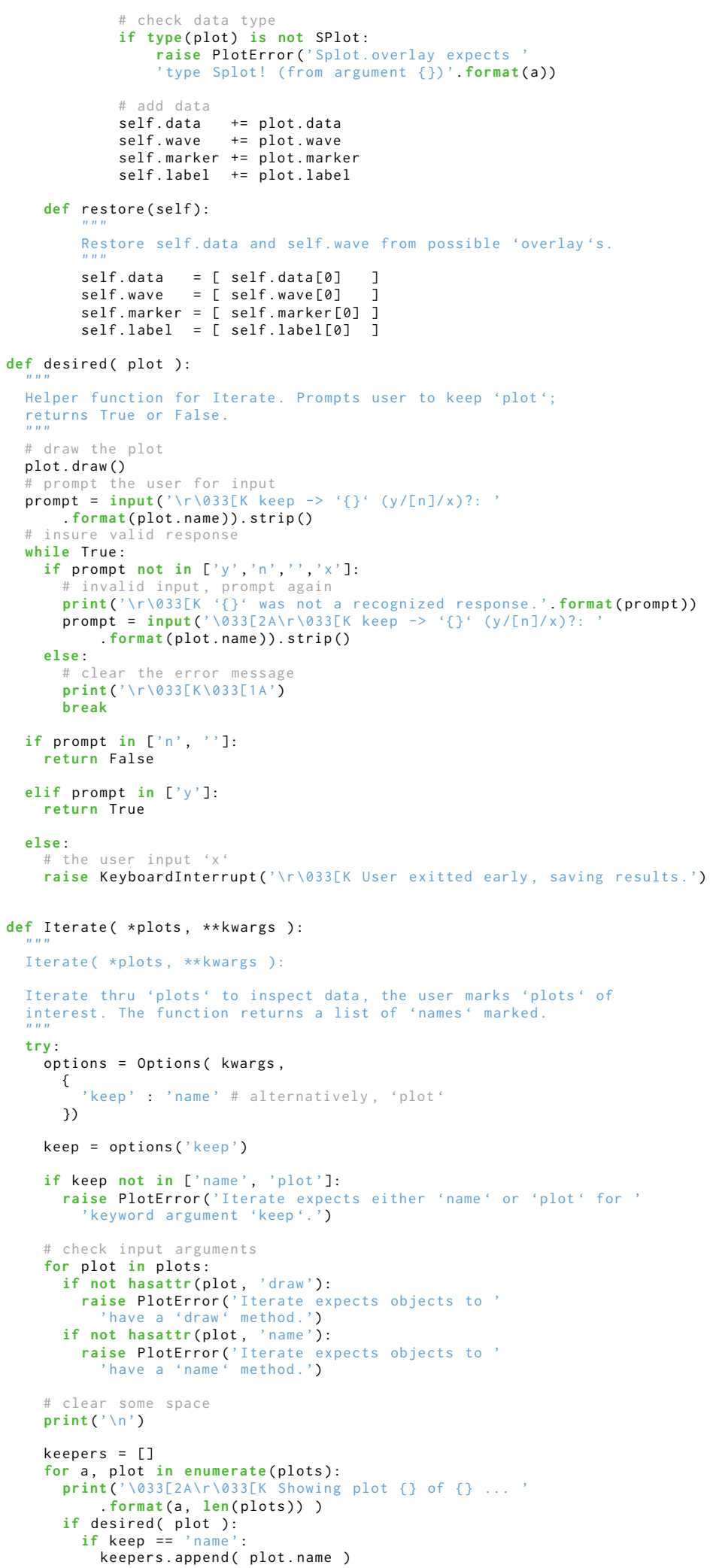




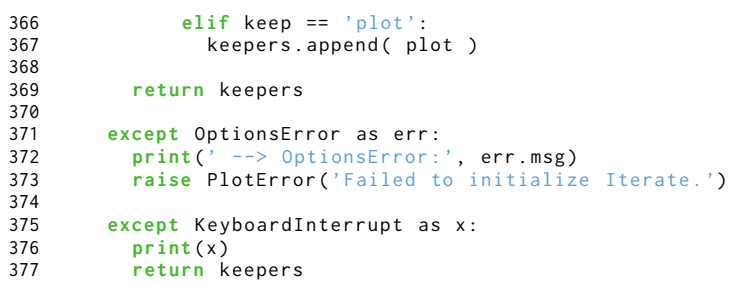




\section{C.16 .. SLiPy . Profile}

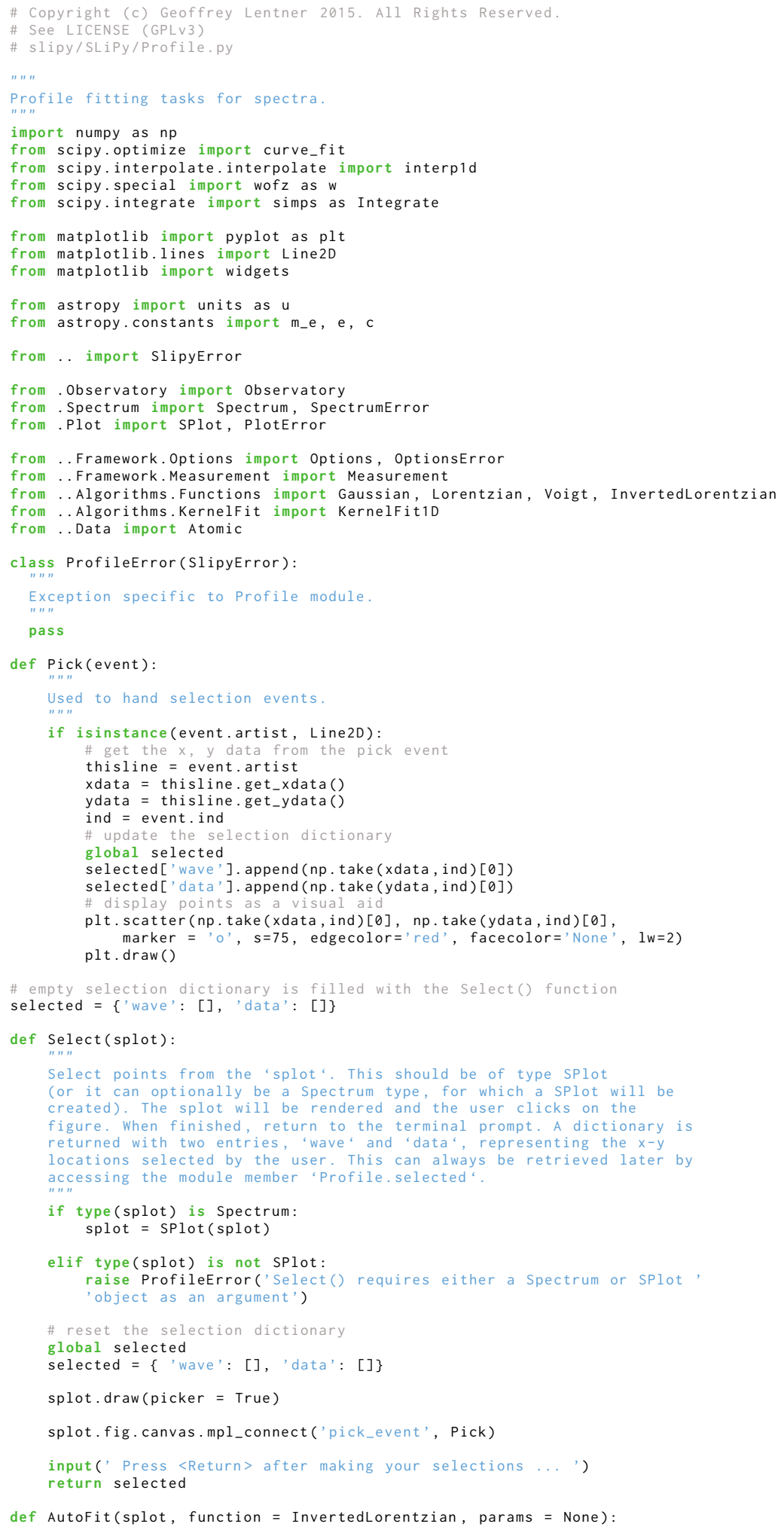




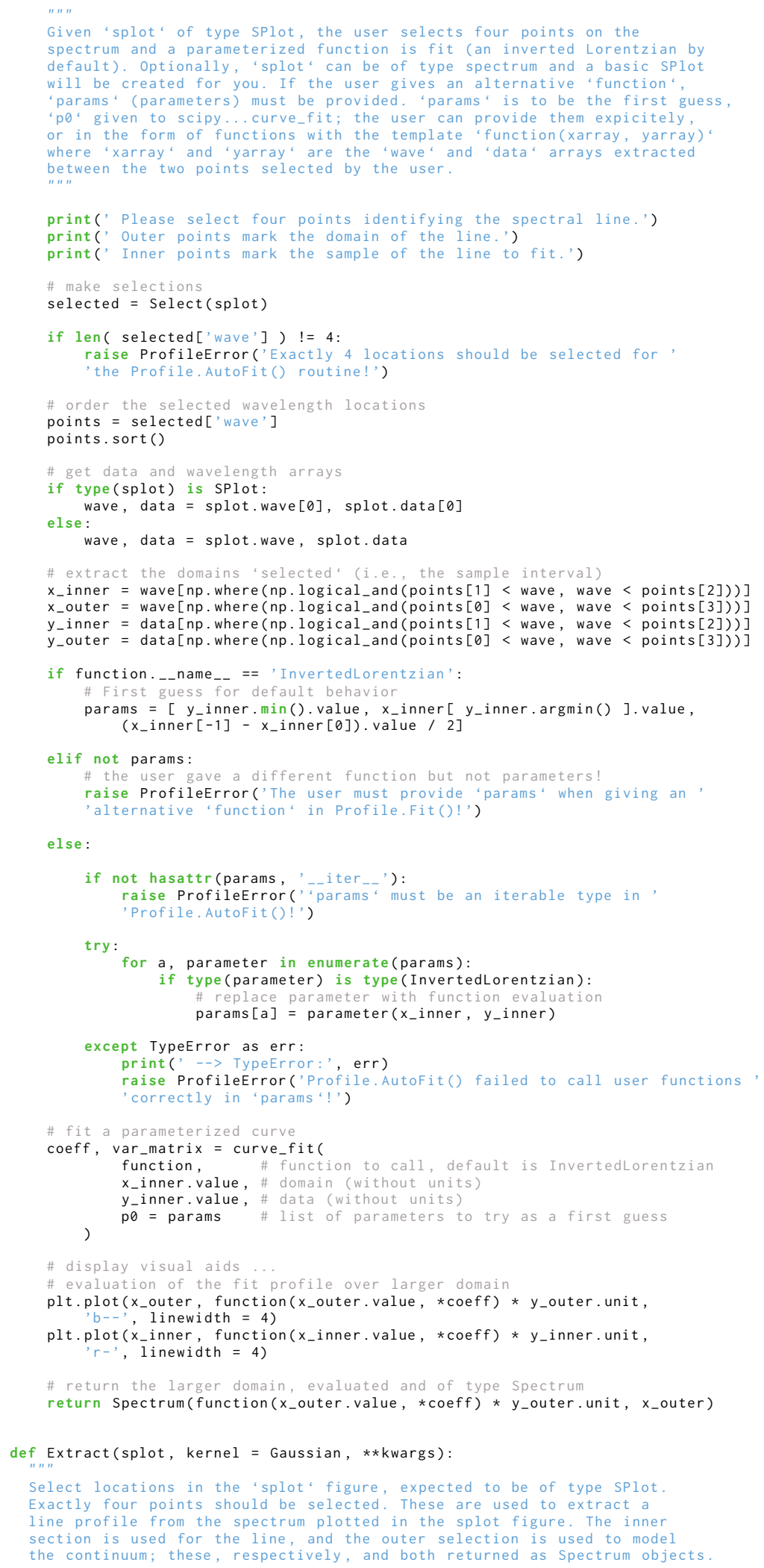




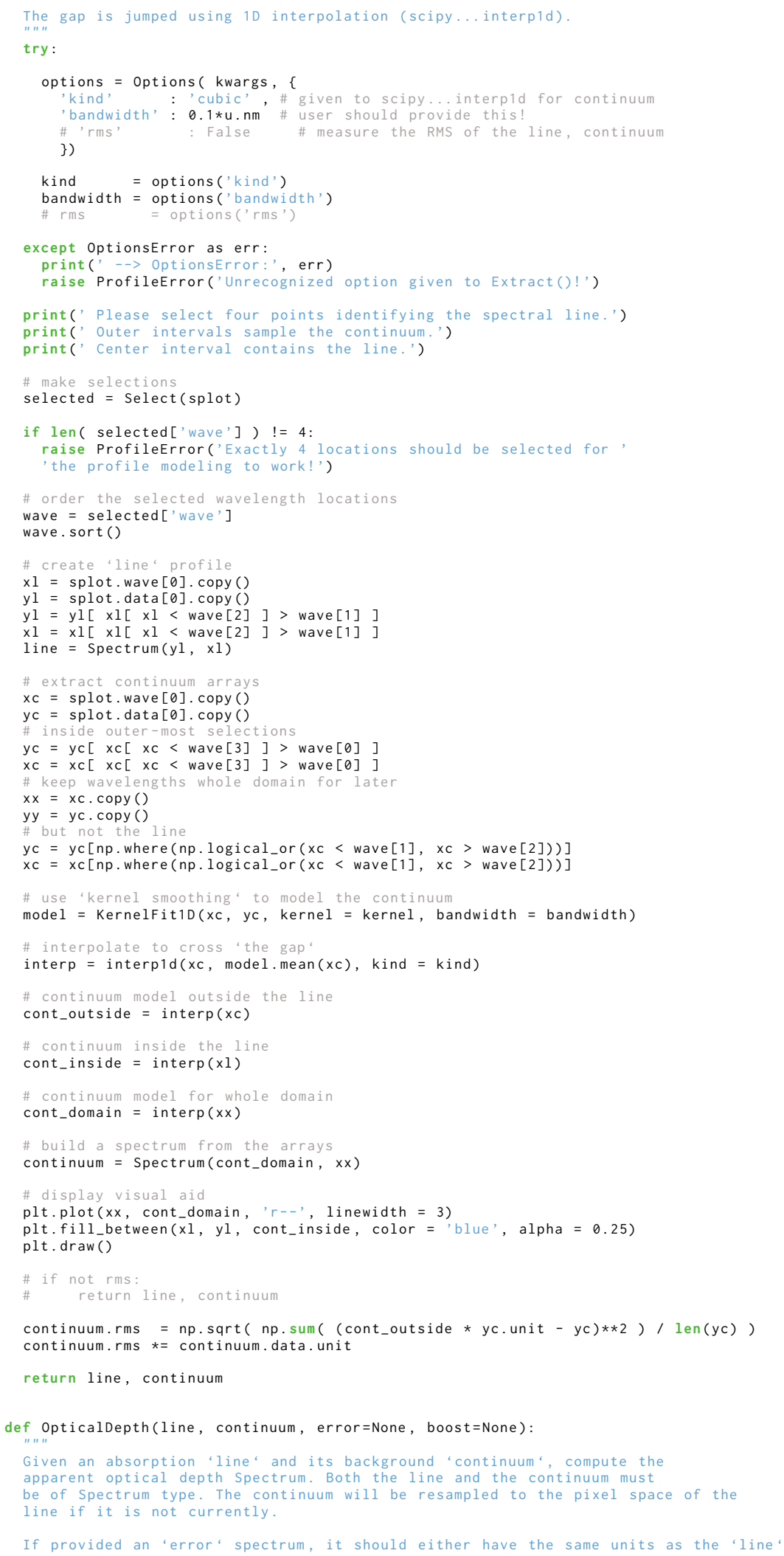




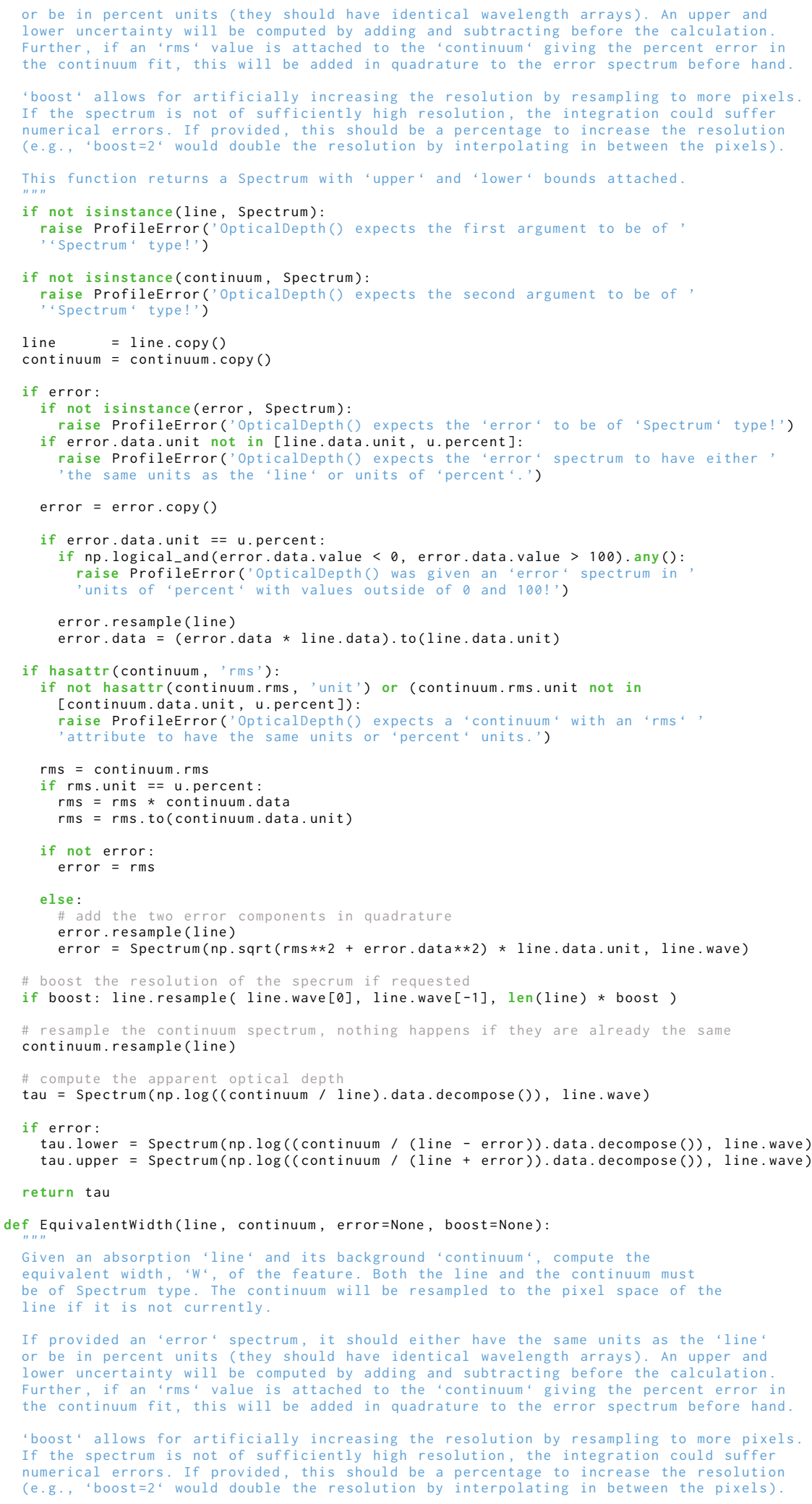




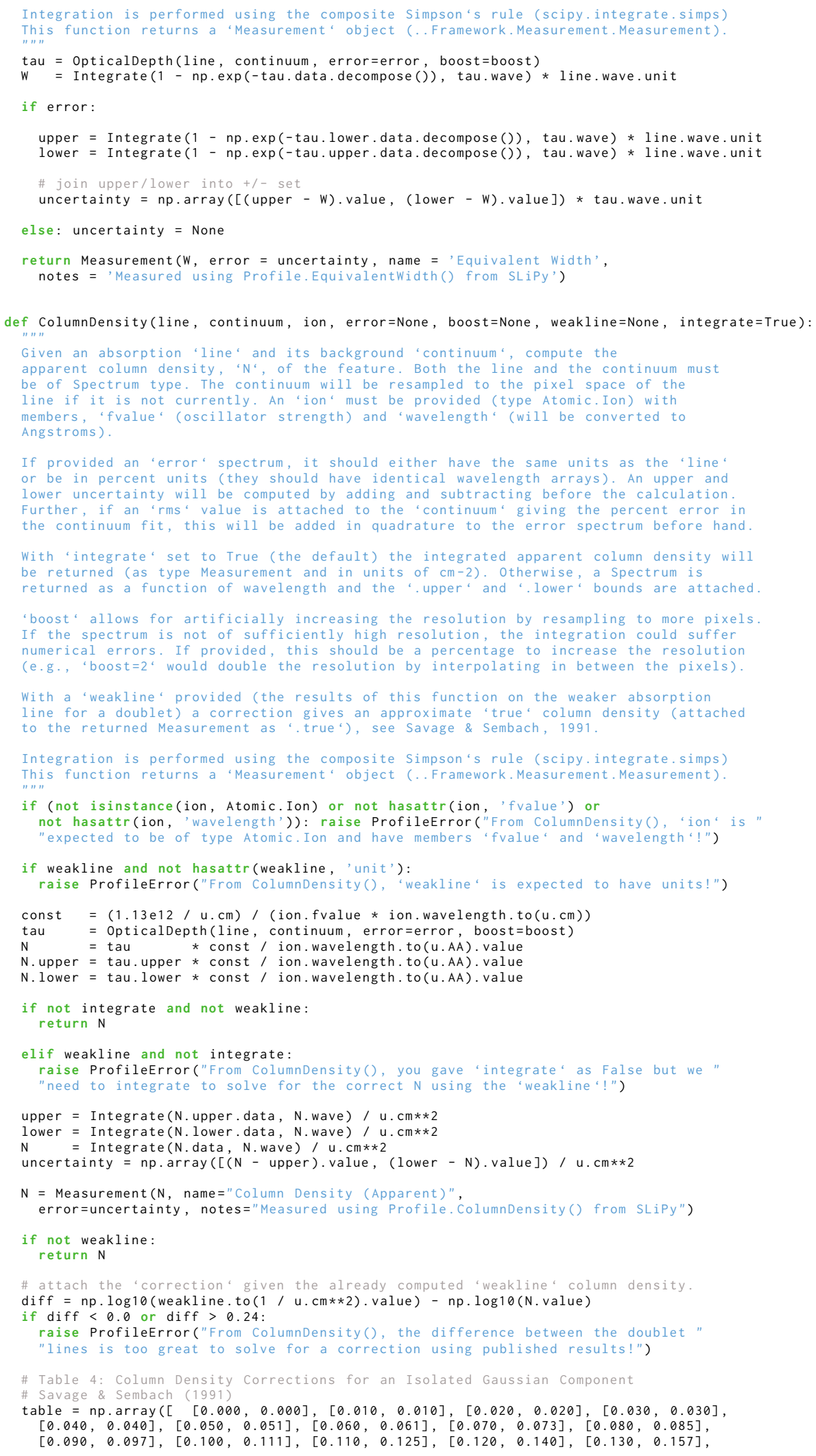




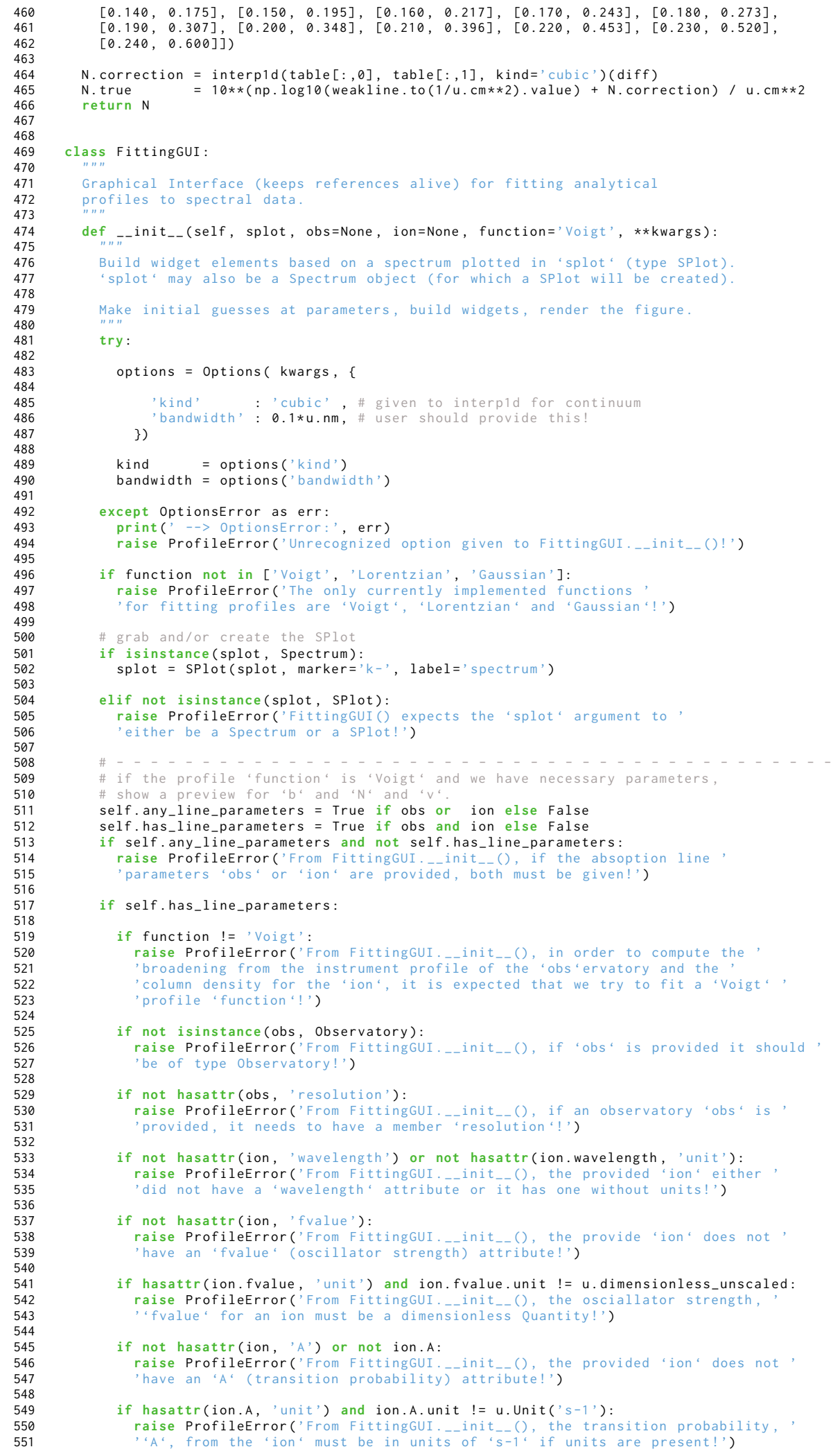




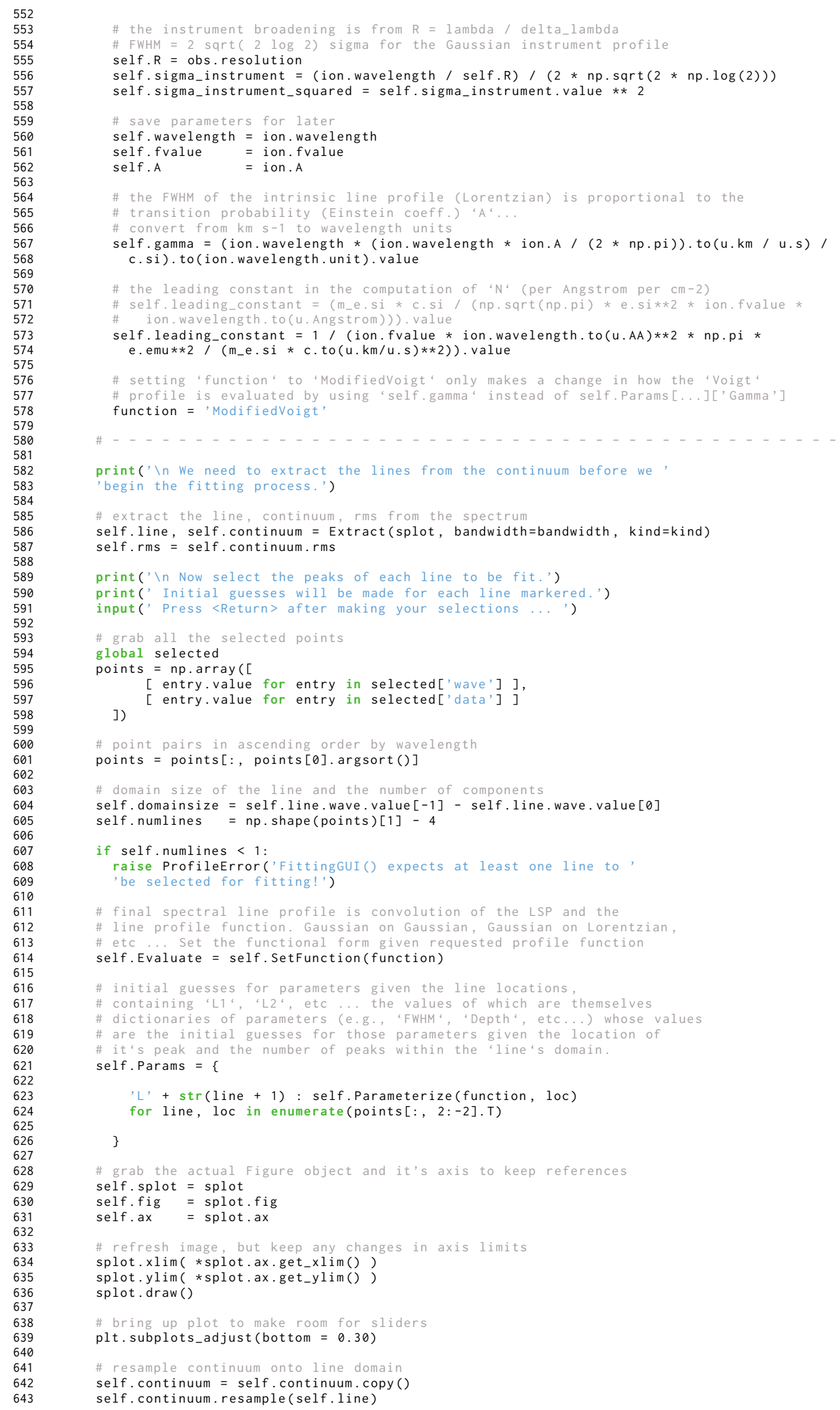




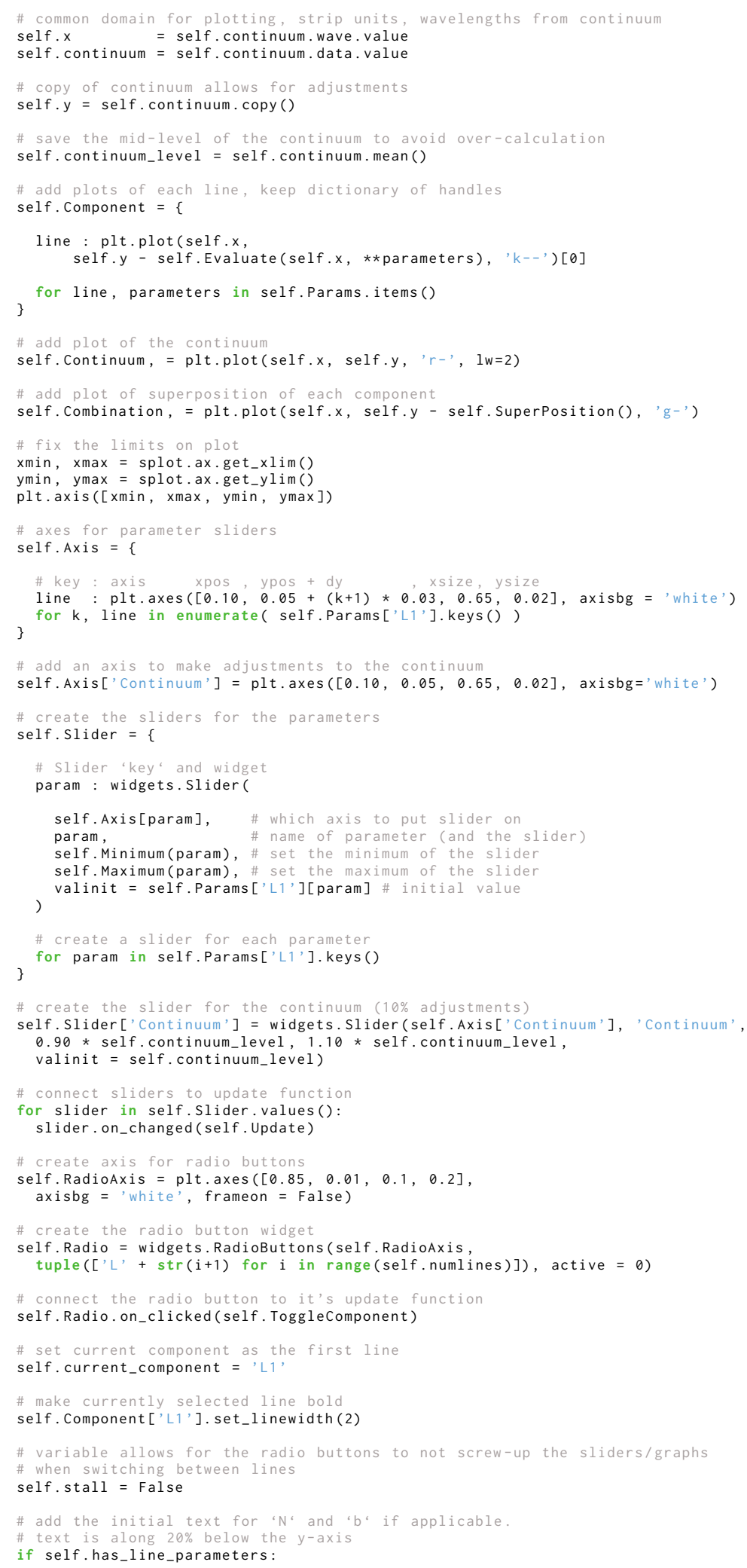




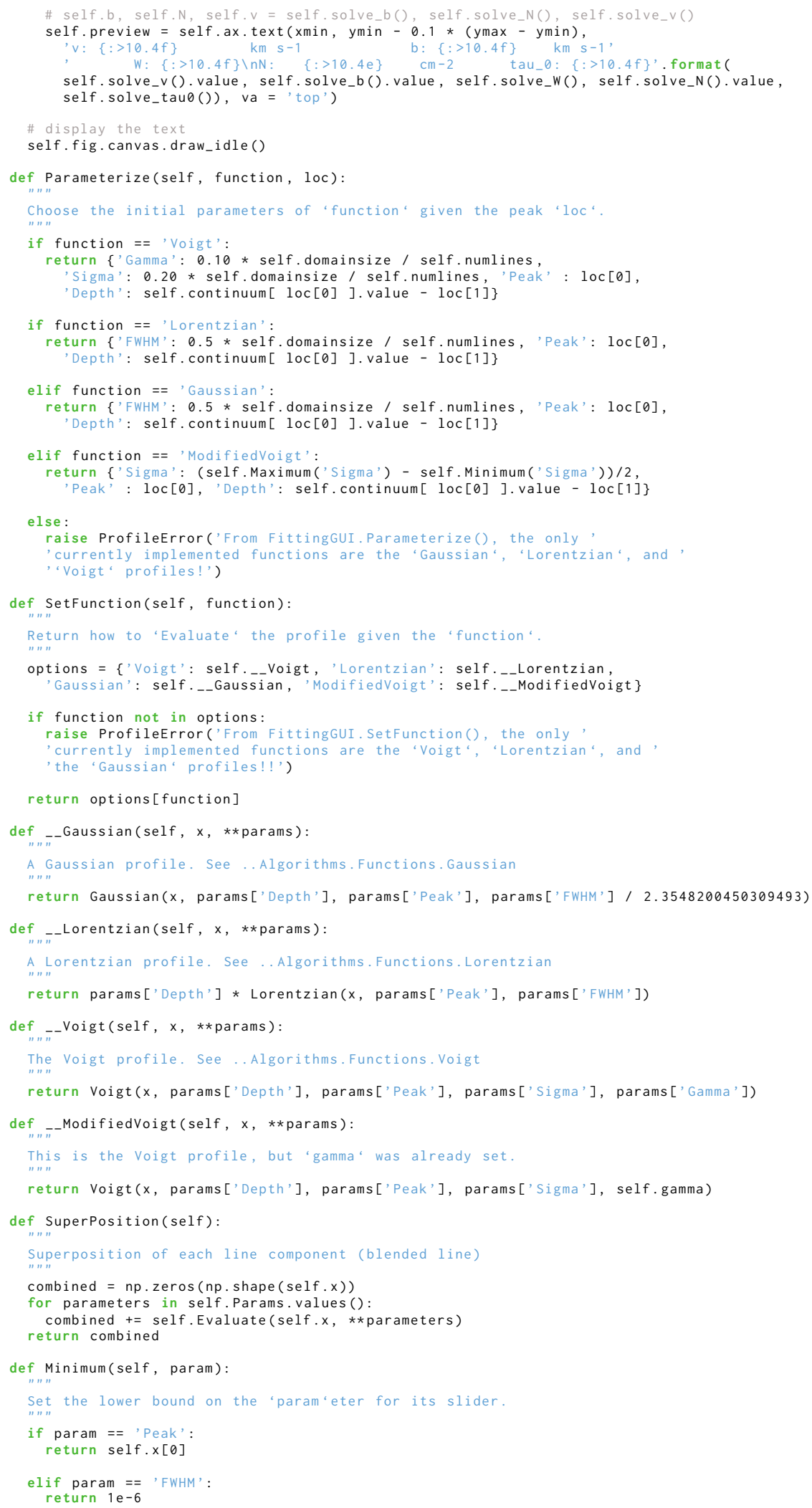




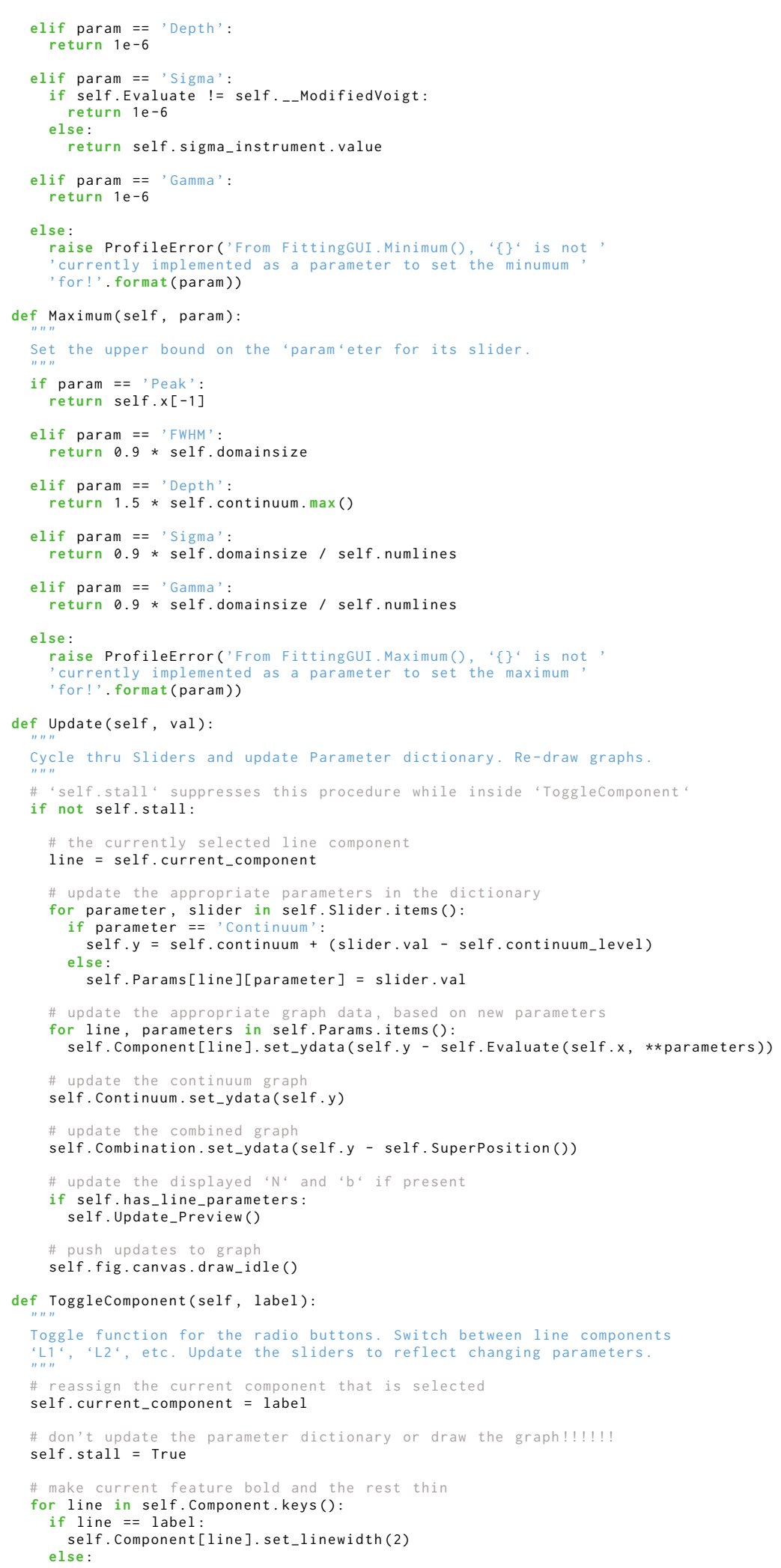




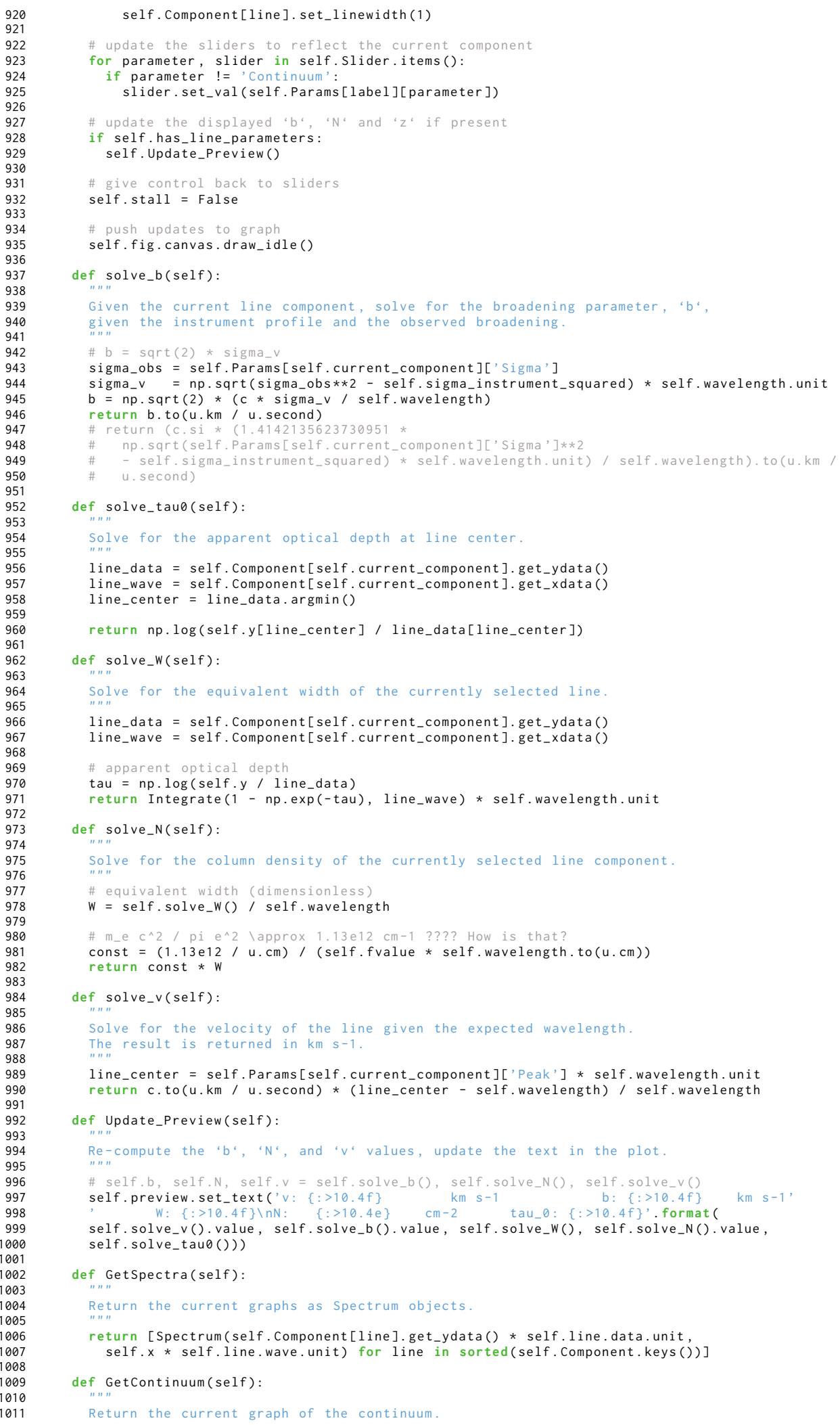




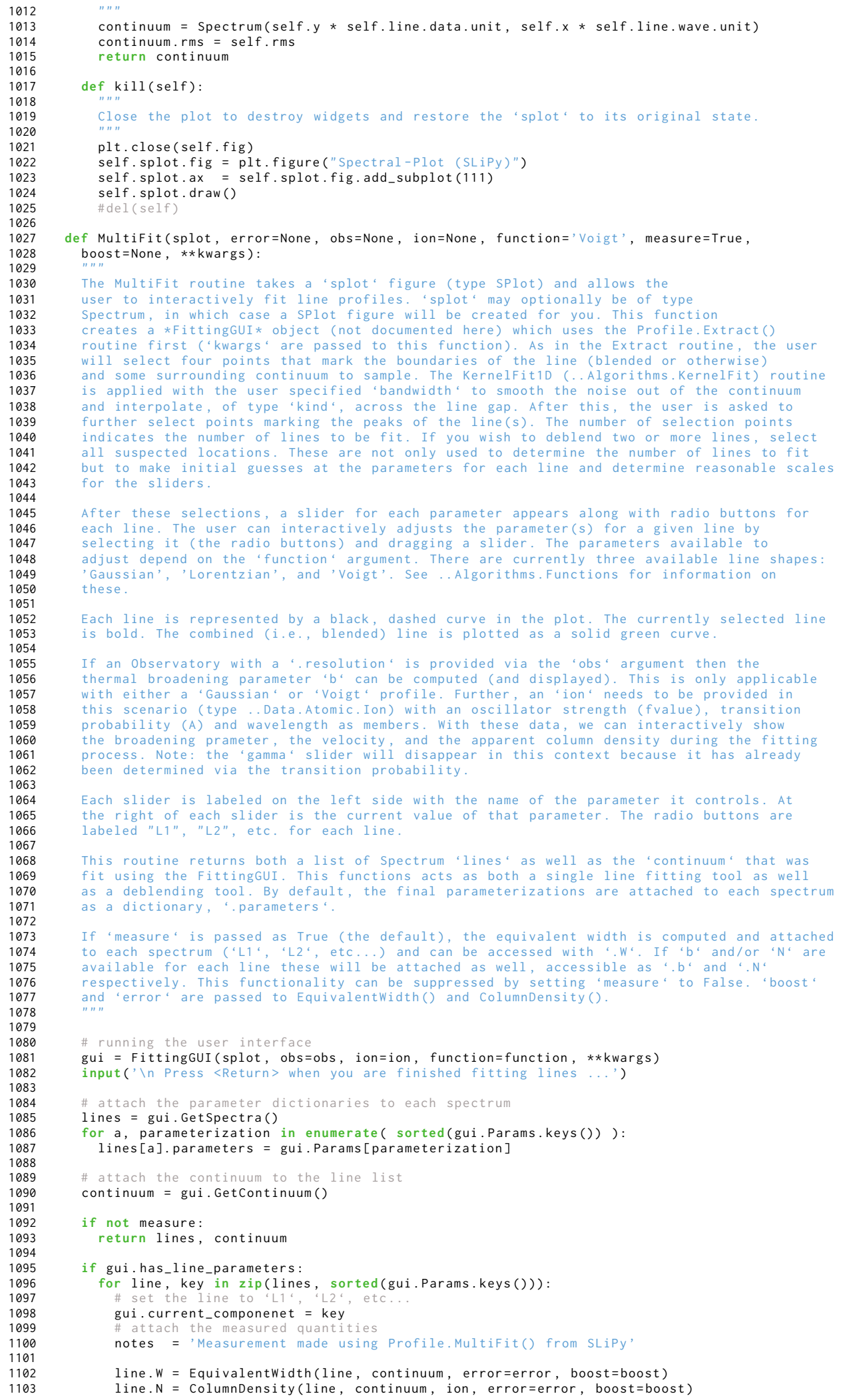




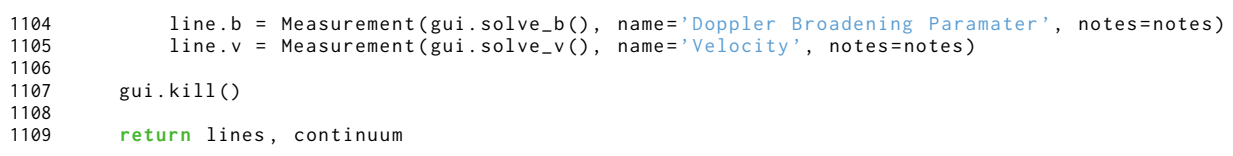




\section{C.17 .. SLiPy . Simbad}

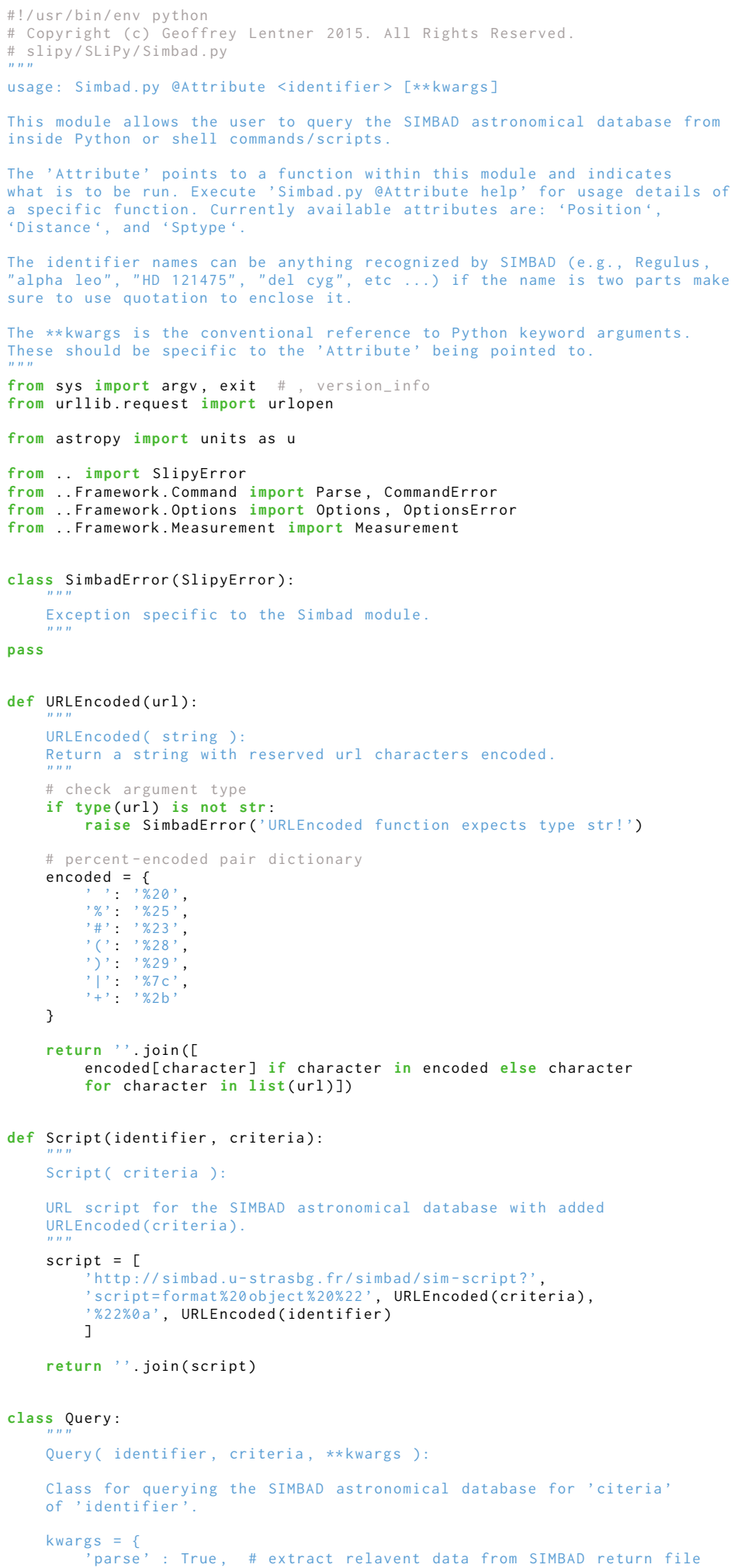




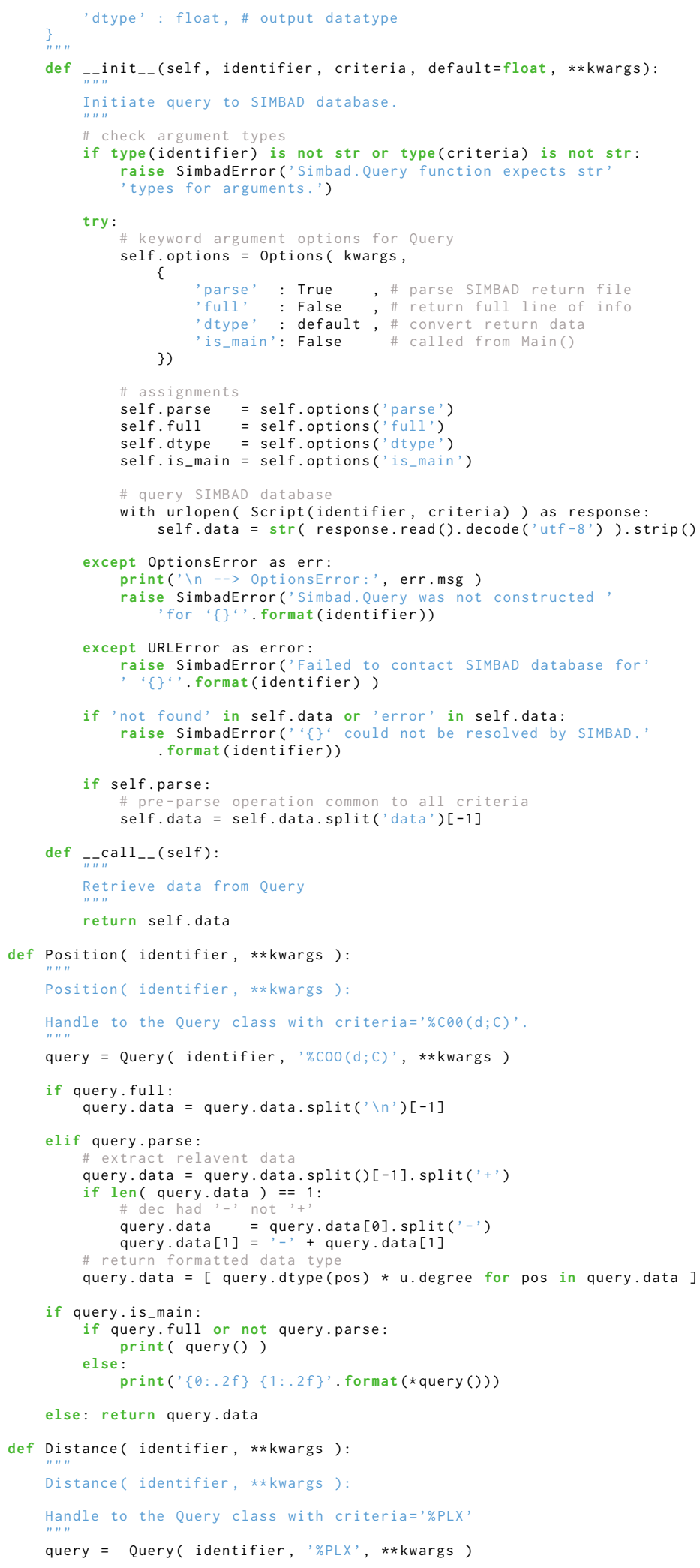




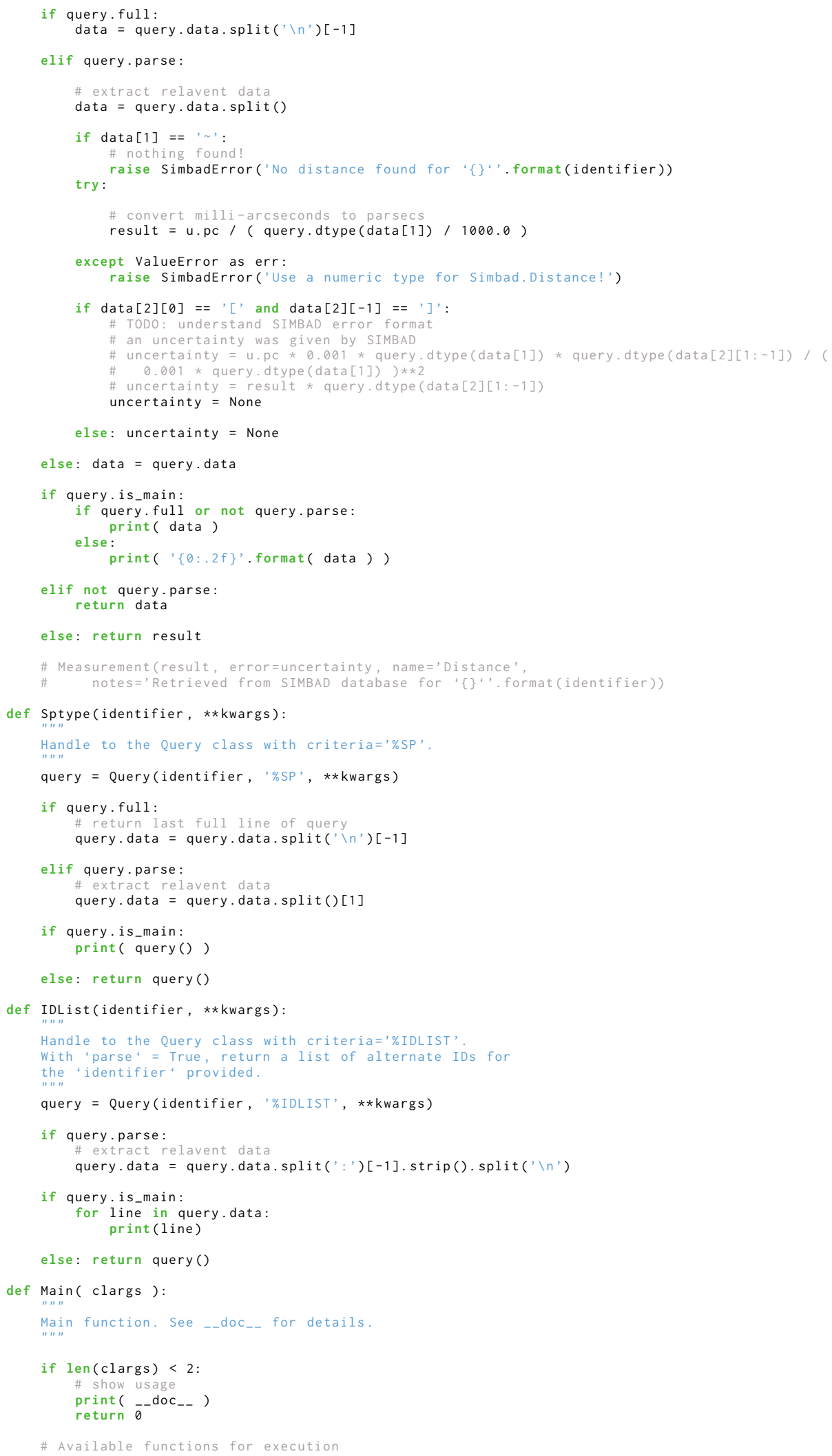




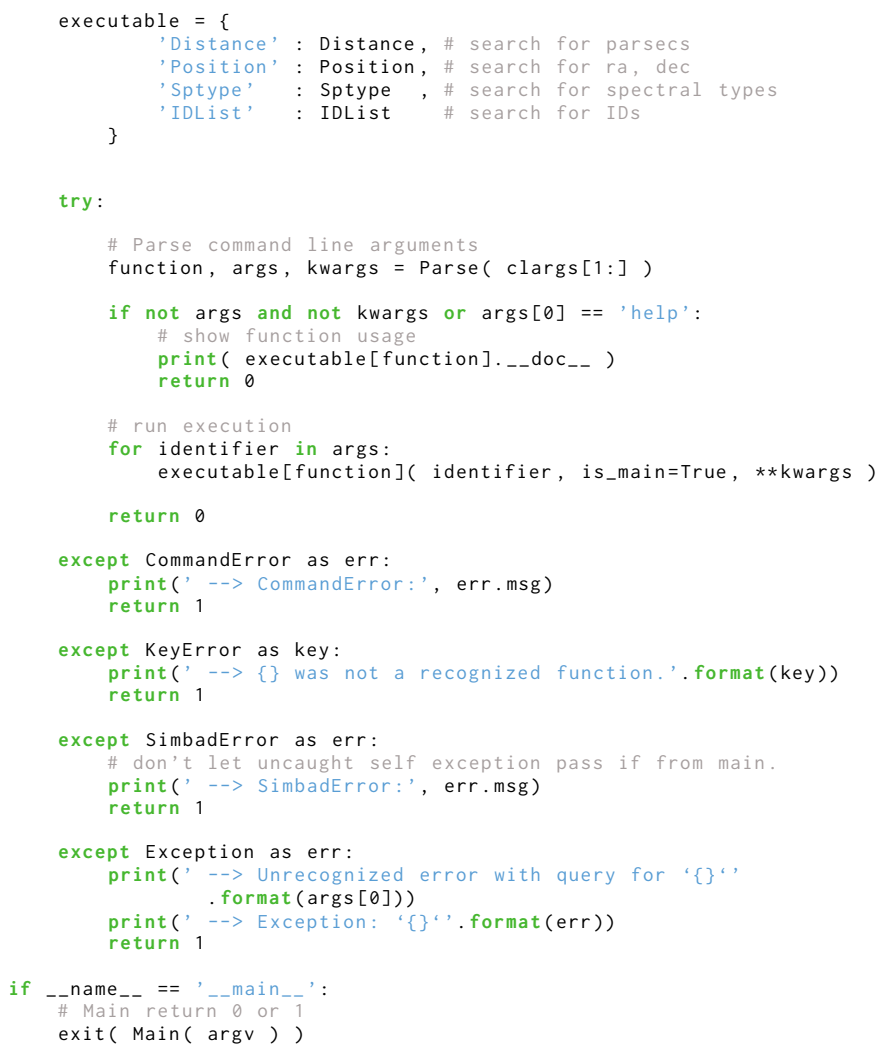




\section{C.18 .. SLiPy . Telluric}

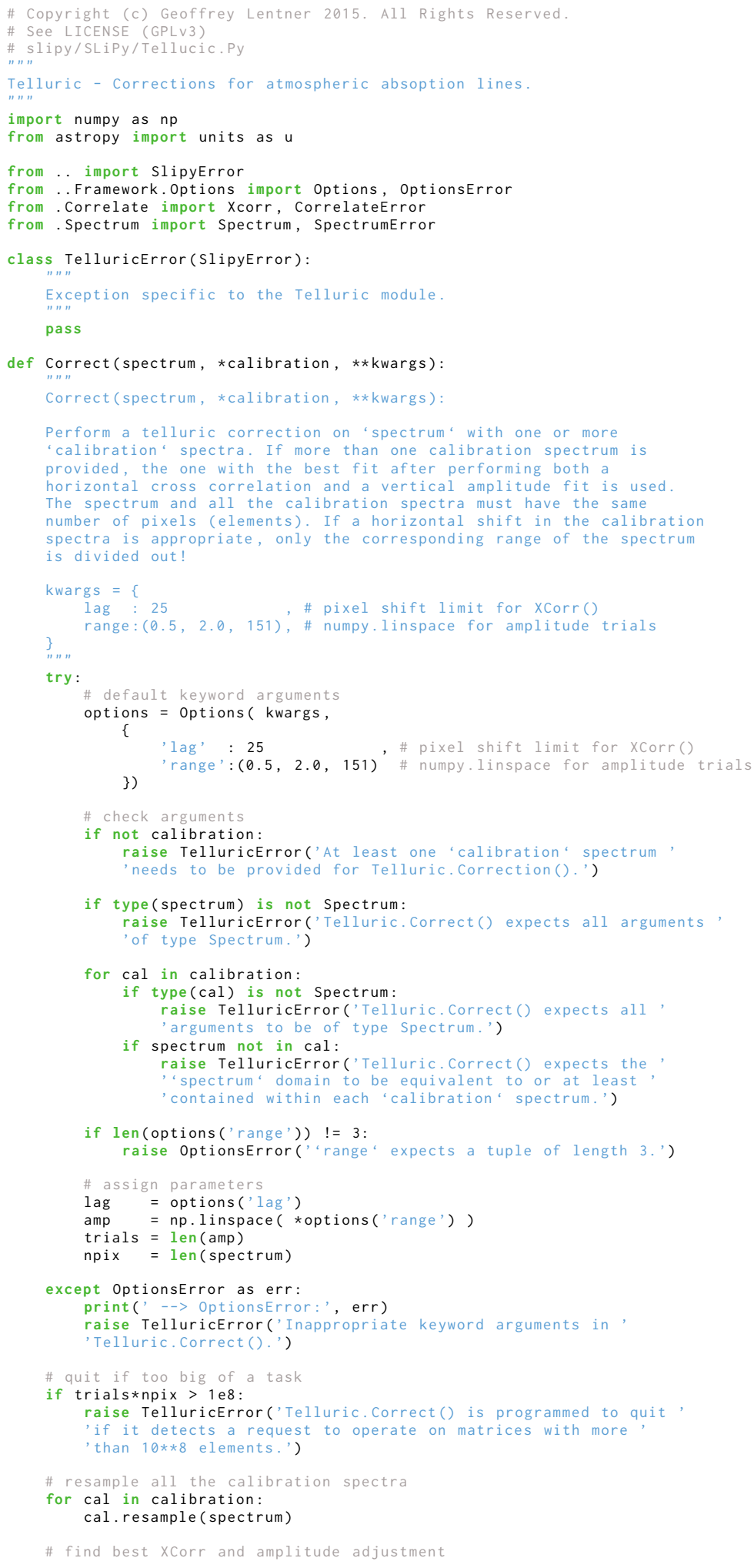




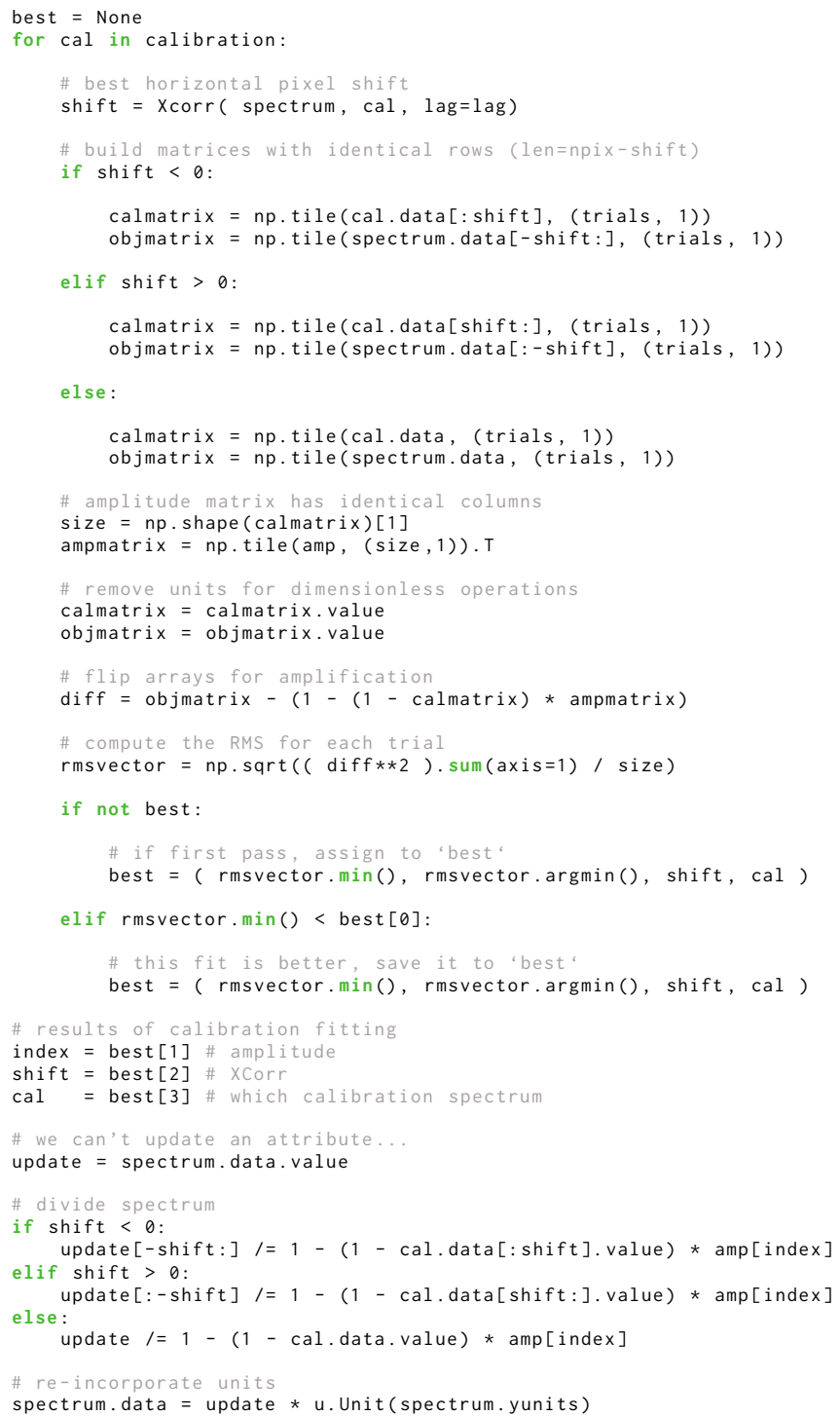




\section{C.19 .. SLiPy . Velocity}

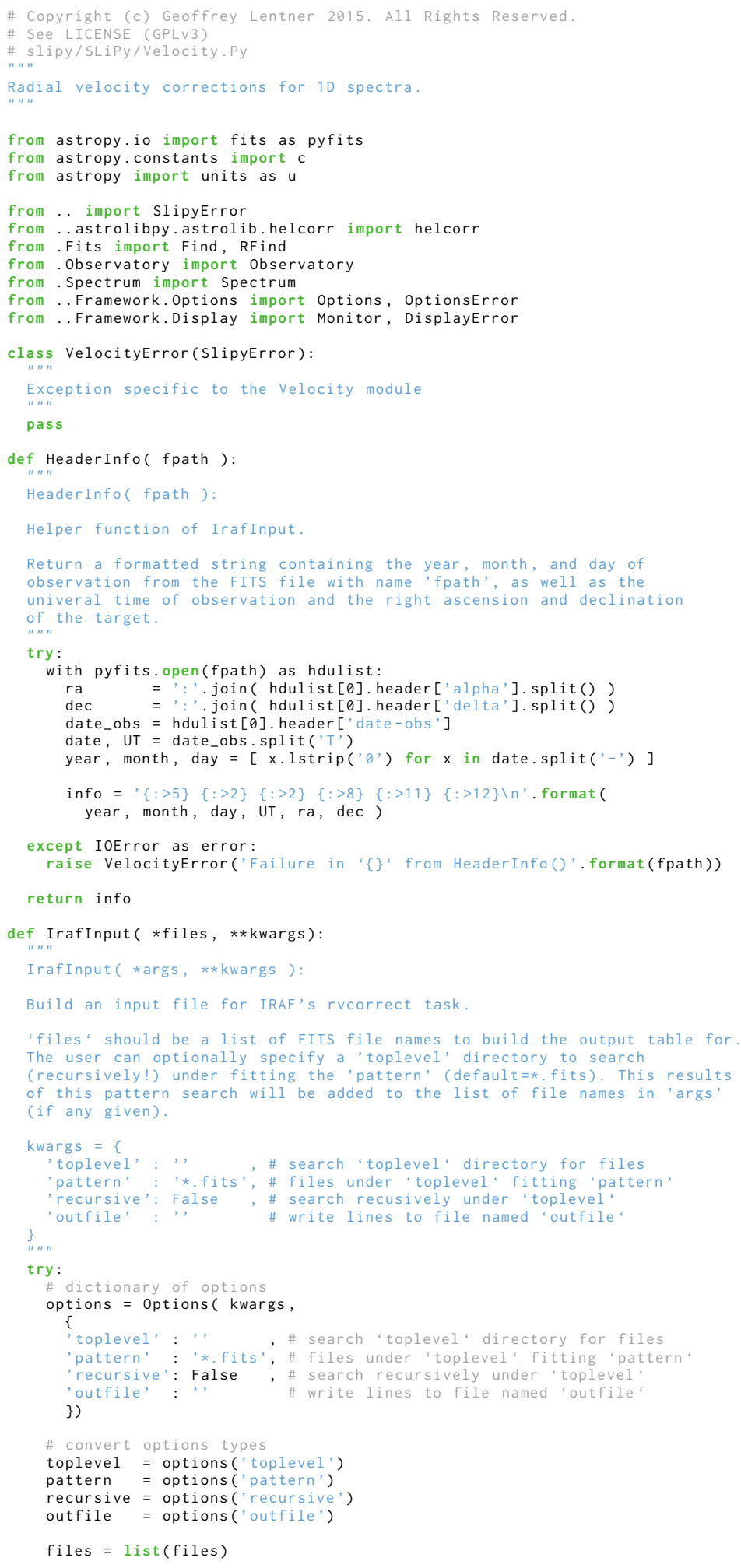




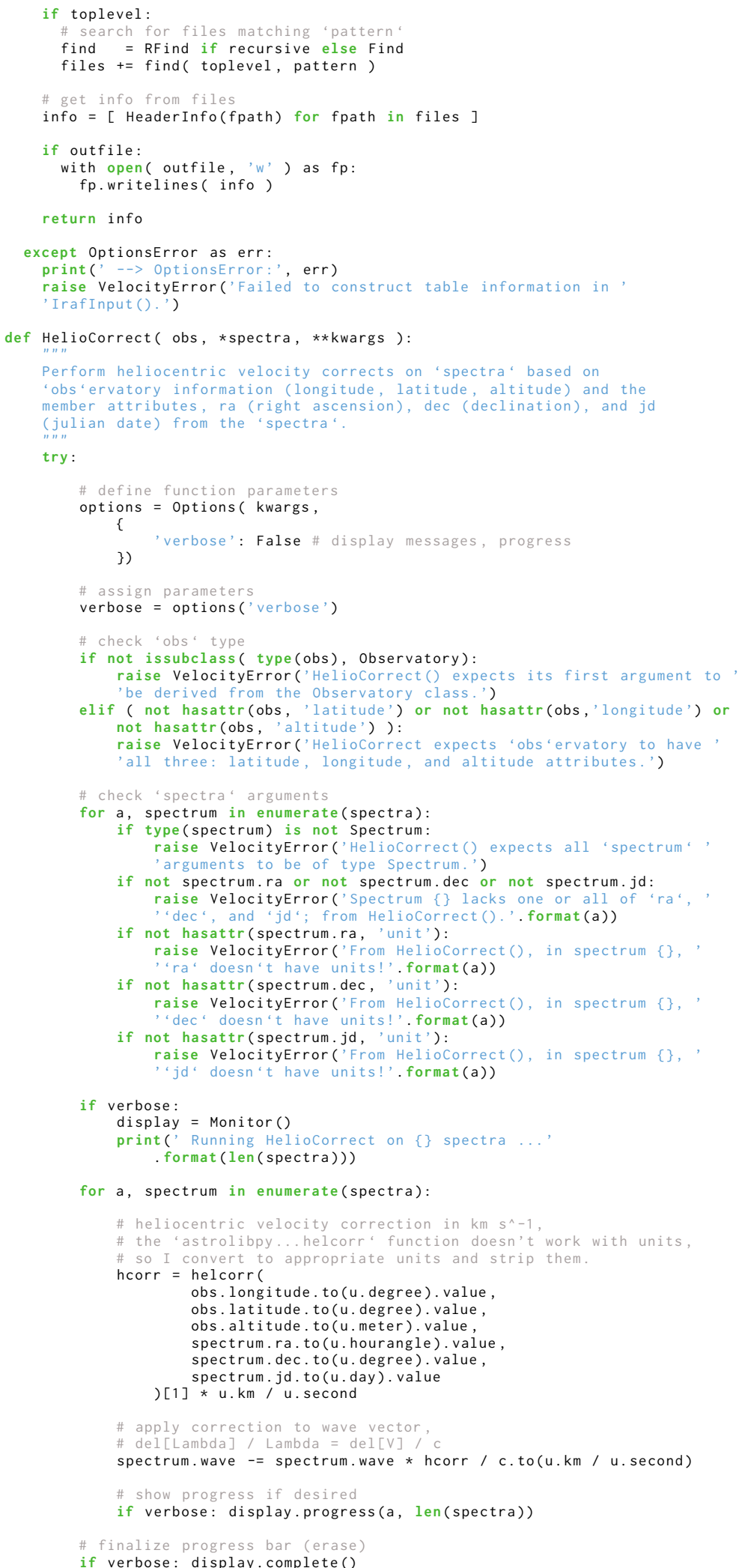




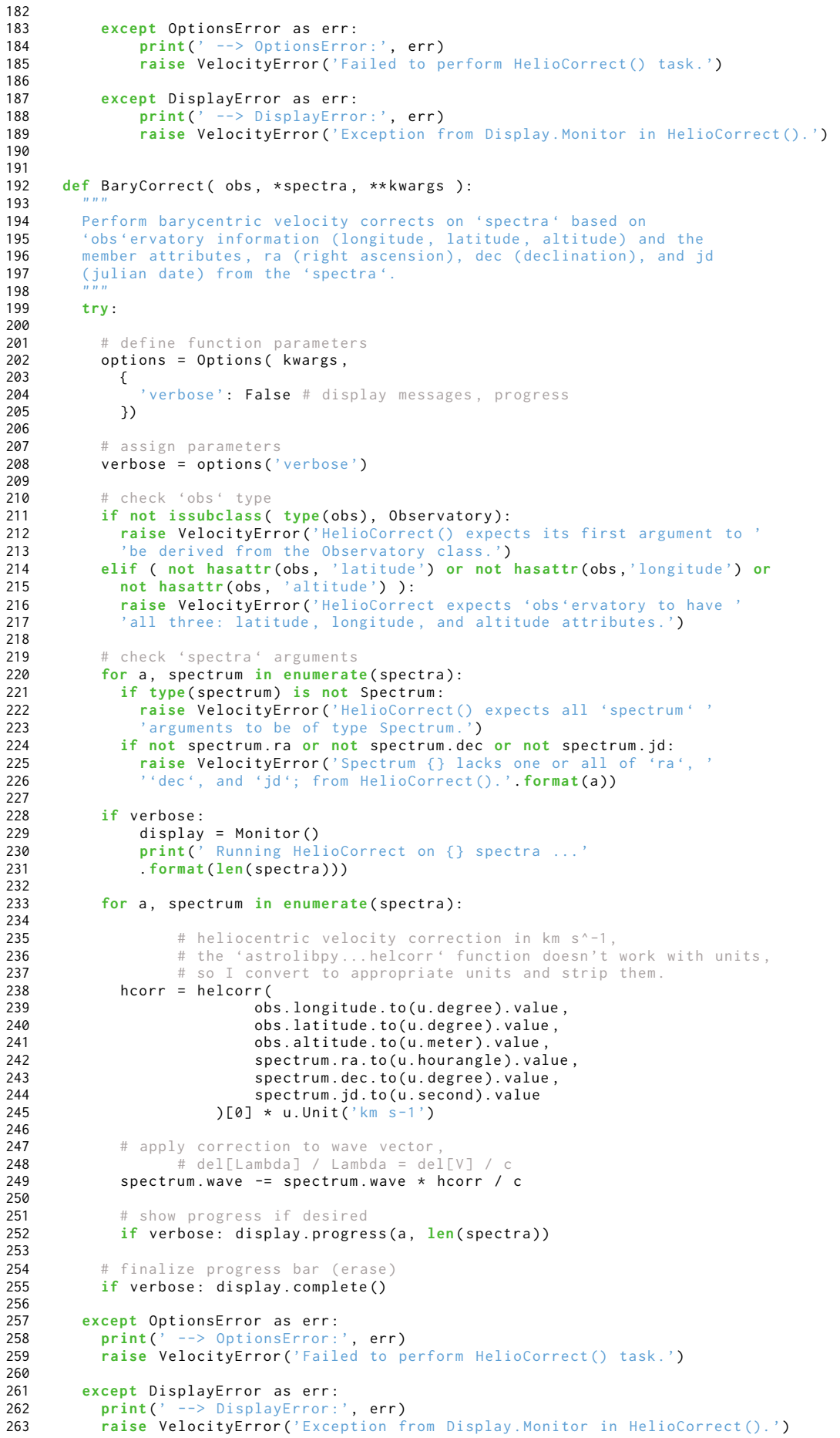




\section{CURRICULUM VITAE}

NAME: $\quad$ Geoffrey Robert Lentner

ADDRESS: Department of Physics

University of Louisville

Louisville, KY 40292

DOB: $\quad$ October 23, 1989

EDUCATION: B.S. Physics and Astronomy

Purdue University

2013 


\section{WORK EXPERIENCE}

\section{University of Louisville}

Graduate Teaching Assistant, 2014-2015

Astronomy \& Astrophysics

Purdue University, PRIME Lab

Undergraduate Controller, 2012-2013

Accelerator Mass Spectrometry

Dr. Mark Caffee (Professor, Director of PRIME Lab)

\section{Purdue University}

Undergraduate Research Assistant, 2011-2012

Experimental Physics

Dr. Rafael Lang (Assistant Professor)

\section{Purdue University}

Undergraduate Research Assistant, 2011

Astronomy \& Astrophysics

Dr. Jaehyon Rhee (Research Professor) 


\section{TEACHING EXPERIENCE}

\section{University of Louisville}

Fundamentals of Physics I (Physics 223)

Elementary Astronomy Laboratory (Physics 108) 